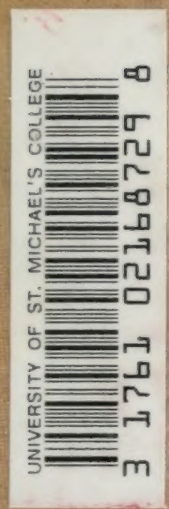




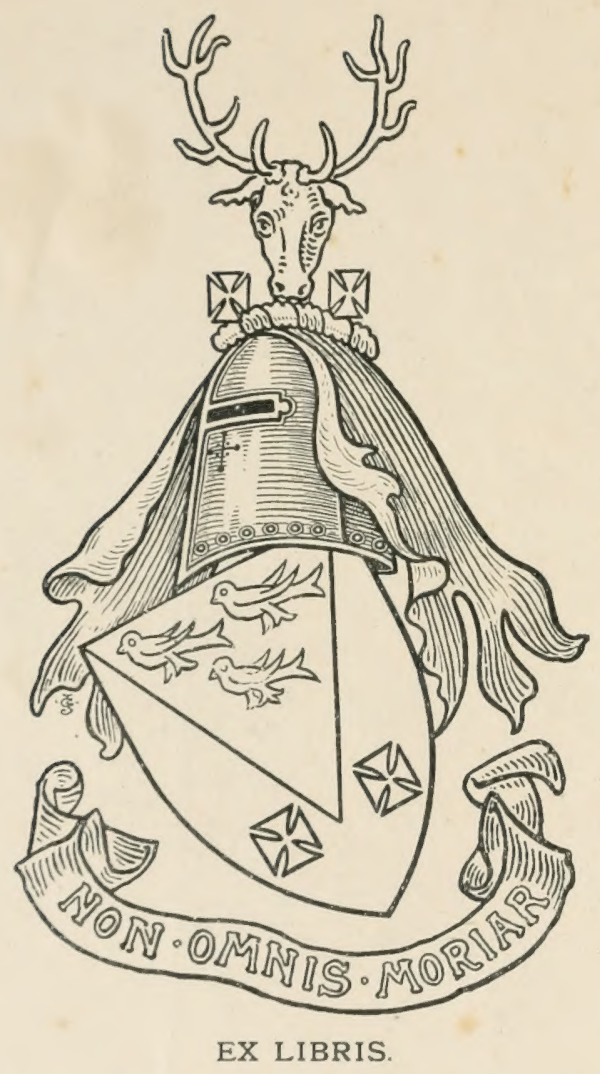

Apertram $\mathfrak{C}$. A. 野inde,

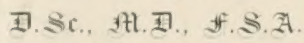




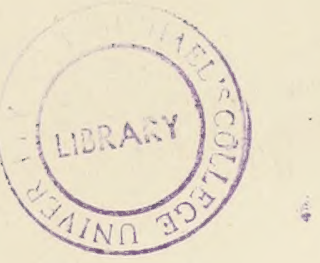


Digitized by the Internet Archive in 2011 with funding from University of Toronto 






\section{Les Problèmes de la Vie}

Essai d'une interprétation scientifique des phénomènes vitaux

par lo

Dr. ERIMANNO GIGLIO-TOS

Professeur de Zoologie, d'Anatomie et de Physiologie comparées à l'Université de Cagliari

II PARTIE

L'ontogénèse ef ses problèmes

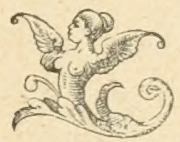

CAGLIARI

CHEZ L'AUTEUR - A L'UNIVERSité

1903 
Ious. droits réservés

Imprimerie Pierre Gerbone - Turin. 


\section{PREFACE}

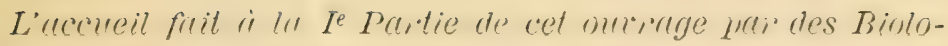
gistes qui possèdent, dans la matière traitée, une compétence alisolument incontestable, a été, sans aucun doute, la récom-

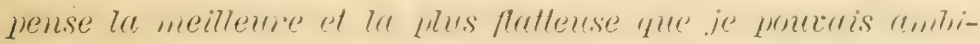

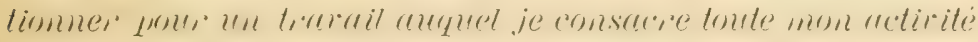
intellectuelle of tonte mule passion scientifique.

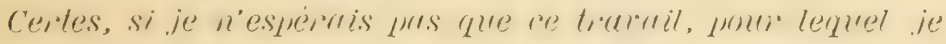

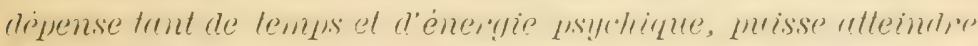

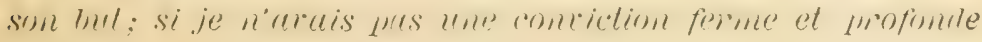
que mon interprétation permel de donner une explication des phénomènes fondameintaux de la vie, en se basant sur des finincipes absolument scientifiques, je n'aurais point entrepris celle publication, qui ne peut obtenir diautre récompense, très

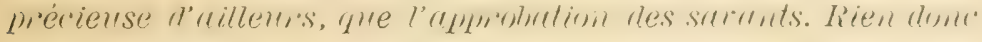

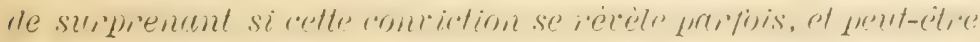
souvent, dans les pages de ce livre, ce qui, du reste, ne saurail altérer en aucune fagon la valeur intrinsèque des idées qu'il conticut.

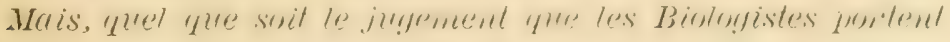

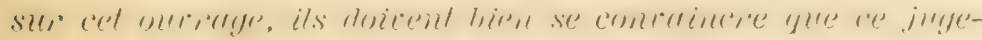

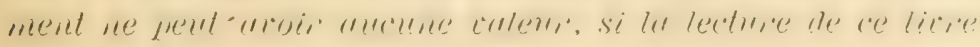




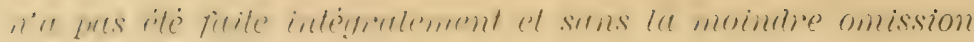

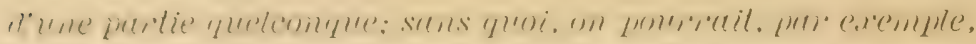

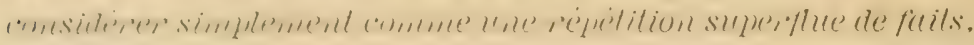

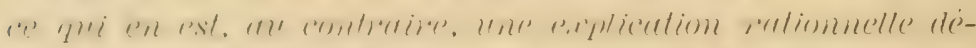

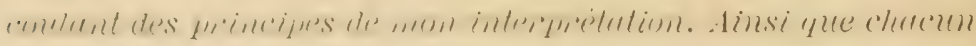

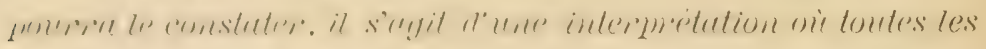

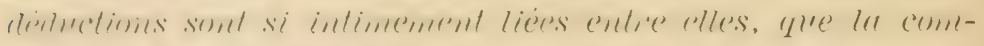

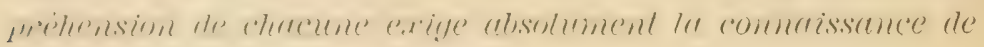
toutes celles qui la précédent.

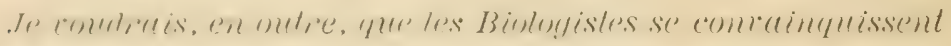

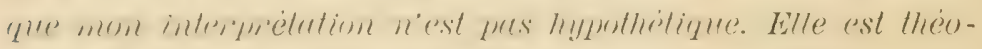

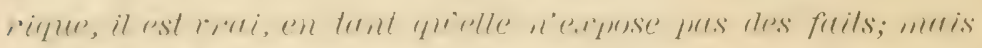

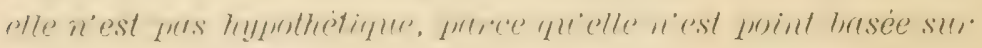
des hypothèses spéciales. Ce n'est pas une hypothèse que le dédoublenent de certuines molécules organiques, sur: lequel se base l'intermintation de l'assimilation; ce n'est pas une hypothese que l'altraction des particules des corps (quelle que soit la cause de celle altraction), sur laquelle je base l'interpiétation de la cylodiérèse; ce n'est pas une hypothèse qu' les volumes des spherres soml entre eux comme les cubes des rayons; el, par ce principe, j'explique la dévialion des plans le division des cellules soumises a une pression.

Ce que j'appelle biomolécule ou molécule vivante, ce n'est pas une molécule à laquelle j'altribue des propriétés sjéciales, par exemple, l'assimilation; mais c'est uniquement une molécule de composé organique, ayant toutes les mopièlés - et pas d'aulies - que les coimposés oiganiques possèdent, enti.e autres, celle de powroi; se dédoubler en dewx molécules égales. Et conme retle jooprèté, ainsi que je l'ai démontré, est dépendante des conditions extinsèques oit les phénomènes de la vie s'accomplissent, j'rpplle biomolécule, la molécule qui jouil de ces propièlés rans les conditions extrinsèques actuelles.

Mon interimétution n'est pas done une hypolhese; elle est, au conticuire, wne méthode sijéciale pour expliquer les pliénomènes

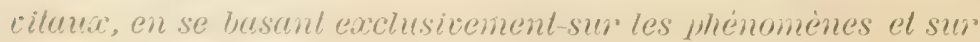




\section{$-\mathrm{r}-$}

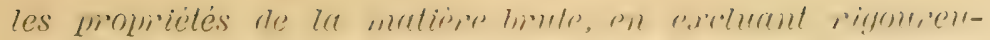

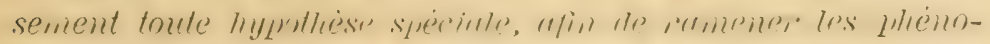

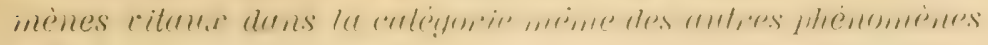

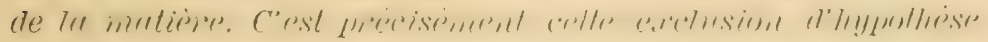

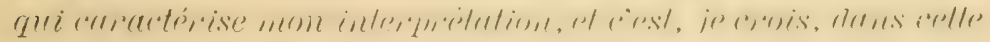

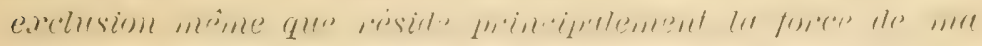

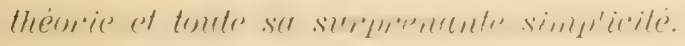

Cagliari, 15 juin 1903.

Dr. Ermanyo Giglio-Tos. 



\section{TABLE DES MATIERES}

PRF́FACE.

Ixtrodcction . . . . . . . . . . . p . . . 1

\section{Chapitre I.}

\section{Les phénomènes fondamentaux de l'ontogénèse.}

SOMMARE: Les phónomènes fondanontaux de l'ontogéaèse - La prolifération cellula:re - La différenciation histologique - La différenciation morphologịue - La losalisation des différenciations - Concomitance de ces phénomènes Examen de la prolifération cellulaire - Examen de Ia différenciation histologique - Histogénèse glandulaire - Histogénẻse des cellules adipeuses, des érythrocytes et des cellules cornées - Histogénèse des tissus conjonctifs et du tissu musculaire - Histogénèse des cellules nerveases - Examen de la différenciation morphologique - Importance capitale du plissement des feuillets germinatifs et importance secondaire du cytotropisme dans la différenciation morphologique - Examen de la losalisation des différenciations - Localisation de la prolifération cellalaire et des différenciations histologique ot morp̧hologique - Résumé. . . . . . . . pag. g

\section{Cilapitre II.}

\section{La différenciation chimique.}

SOMMAIRE: La hitférnciation histolegique est une sicrétinu - La nature glandulaire de toutes les cellules - La sécrétion est lo seul et le vrai caractèro de la différenciation histologique - Toutes les cellules vivantes ont leor diffürenciation histologiquo - Les propriétés les substances sécrétées ne sont pas des caractères d'importance dans la différenciation histologique - La diversité de constitution chimique des substances caractérisant la différenciation histologique - Importance de l'arrangement atomique dans les propriétés physiques, cbimiques et physiologiques des substances - Différences dans la constitution chimique des substances possédant les mêmes propriétés physiologiques - Ia cause dos différenciations histologique et morphologique est la différenciation chimique - La cause de la localisation des différen. ciations est la localisation de la différenciation chimique - Résumé pag. 23 


\section{('IIITTRE: III.}

\section{I'oeuf.}

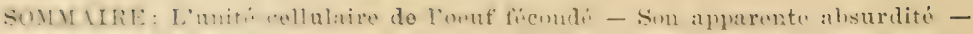

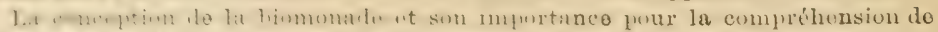

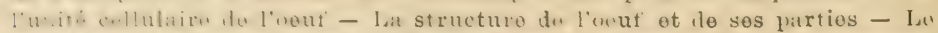

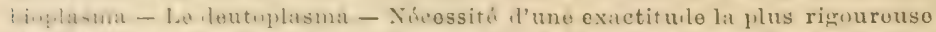
duns la listinction los purties wrulaires - Le vitellus formatif ot lo vitellus

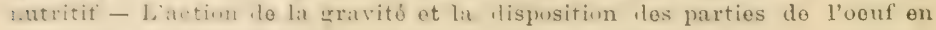
raison do lour densité - L'isotropio do l'oouf - Résumé . : pag. 41

\section{Chapitre IV.}

\section{Les bases possibles de lontogénèse.}

SOMMAIRE: Existence dans l'oouf des conditions chimiques nécossairos pour l'assimilation - Nécessité des conditions physiques favorables - Importance ot rôle du spermatozoïde dans la fécondation - Les bases possibles de l'ontogénése: les léveloppements biomoléculaires autogénétique, homogénétique, Tuétérogénétique - Examen de ces đẻveloppements - Insuffisance des développoments autogénétique et homogénétique pour l'explication des pluénomènes ontogénétiques - Exclusion de ces modes de développement - Le développement hótórogénétique est la baso de l'ontogénèse - Résumé pag. 50

\section{Chapitre V.}

\section{Le développement hétérogénétique.}

SOMMAIRE: L r potentialité évolutive do l'oenf - Son origine et ses limites Les phasos do l'évolution de l'oouf - Le développement polyodique - Ses consérauences et son insuffisanco à l'explication des phénomènes ontogénétiques - Le développement monodique - Ses conséquences et son importance dans l'ontogènèse - Résumé pag. 59

\section{Chapitre VI.}

\section{Le développement monodique.}

SOMMAIRE: L'évolution de l'oeuf dans lo développoment monodique - La ra. pidité de cetto évolution - La phase limite ot les phases intermédiaires L'hétérogéneité de l'agrégat cellulaire résultant de la segmentation - Son retour possible it l'homogénéité - Lá déviation des cellules de leur évolution primitive - Résumé

pag. 68

\section{Cimapitre VII.}

\section{L'asynchronisme de segmentation.}

S()MMAIRE : Imposs:bilitó du symchronismo parfait dans la division des blastumirrs - Causes do cetto impossibilité - La duréo de la périodo assimilatrico - La duréc do la púriodo cytodiérésiquo - L'asynchronismo accéléré ot l'asynchronismo ralenti - Etruts de l'asynchronismo accélérỏ et du développemont mmorique - La segmentation-de l'oeuf - La production dos sillons - Lo rythme de srgmentation - La polarité nt la symétrio bilatérale de l'agrégat collulaire - Les collules homonymes contemporaines - L'accólération de la segmentation fénéralo - L'asynchronismo ralonti ot ses offots - Lo ralontissement do la sogmentation généralo - Résumé . . . . 1"'!r. Ty 


\section{Chapitre VIII. \\ La première phase de l'ontogénèse.}

SOMMAIRE: La spécificité ovulaire - La limite ly la potentialité évolutive ilo l'oeuf - La premjère lignée de cellules - L'entrecroisoment des plans de segmentation et son importance dans la formation de l'agrégat collulaire La production de substances de sécrétion de la part des blastomères - La parenchynula - La morula - La blastula - Les conditions chimiques, physiques ot mécaniques agissant sur la formation do la blastula - Action des substances accumulées dans la cavité de la blastula sur la direction des plans de segmentation - Importance secondairo do la forme de l'agrégat cellulaire, et importance capitale de sa constitution - Résumé . pag. 119

\section{Chapitre IX.}

\section{La deuxième phase de l'ontogénèse.}

SOMMAIRE: L'oeuf et son miliou interne - Orizine dece milieu - La probiose de l'ovocyte, et son importance dans la segmentation de l'oeuf - La probiose des blastomères et la création du milieu interne de la blastula - Rôle de ce milieu dans la production de la denxième phase de l'ontogénèse - La deuxième lignée de cellules - La différenciation histologique accompagnant la prolifération cellulaire - La différenciation morphologique - La gastrulation et ses causes mécaniques - La localisation des différenciations - Résumé. pag. 134

\section{Chapitire $\mathrm{X}$.}

\section{L'origine de la symétrie rayonnée.}

SOMMAIRE : La protuction des lignées de cellules de la deuxième phase ontogénétique - La localisation de ces différentes lignées de cellules - L'asynchronisme accéléré et ses conséquences - L'asynchronisme ralenti et l'origine de la symétrie rayounée - Conclusions générales - Résumé - pag. 150

\section{Chapitre XI.}

\section{L'origine de la symétrie bilatérale.}

SOMMATRE : Effets possibles de la probiose des cellules de la première lignée - I'asynchronisme accéléré - La polarité de l'embryon - La symétrie bilatérale - Causes de cette symétrio - Préoxistence de la symétrio de l'embryon dans l'óeuf - Symétrie de l'agrégat cellulairo de segmentation ot symétrie de l'embryon - Rapports entro le plan do symétrio et les deux premiers plans de segmentation - Epoque de l'apparition do la symétrie bilatérale - Résumé .

\section{Chapitre XII.}

\section{Les phases ultérieures de l'ontogénèse.}

SOMMAIRE: Les principes de mon interprétation et lear examen - La symétrie de l'organisme et les symétries do ses partios - La complication progressive de l'embryon et de son milieu interne - L'automatisme des phénomènes ontogénétiques - L'organisme comme système symbiotique - Le fonction- 
nomont des organos ot la vitalité de l'organisme - L'équilibre symbiotique de l'organisme et ses conditions - Le renouvellement incessant de l'organisme - La mort - Les limitos de l'existenco de l'individu - Le rôle des stimulus physiques dans la détormination des différenciations - La localisation dans le tomps ot dans l'esprace des différenciations histologique ot morphologique.

pag. 181

\section{Chapitre XIII.}

\section{Les problèmes de l'ontogénèse.}

SOMMAIRE: § I: Sur lo dóveloppement de portions d'oeuf (I Problèmb) - § II: Sur lo développement des blastomères isolés (II, III, IV Problèmes) - § III: Sur le développement de groupes de blastomères (V Problème) - § IV: Sur le développement de portions de blastula (VI Problème) - \$ V: Sur le développement d'extraovats (VII Problème) - \& VI: Sur le développement de blastomères imparfaitement isolés (VIII Problème) - § VII: Sur les résultats de la destruction de blastomères (IX, $\mathbf{X}, \mathbf{X} \mathbf{I}$, XII Problèmes) - § VIII: Des effets de la compression sur le développement ontogénétique (XIII, XIV Problèmes) - \$ IX: De l'action de la gravité sur le développement ontogénétique - $\mathrm{X}$ : De l'action des agents physiques et chimiques sur le développement ontogénétiquo

\section{Chapitre XIV.}

\section{Le développement mixte.}

SOMMAIRE: Les autres développoments hétérogénétiques possibles - § 10: Les développements dimonodiques et leurs relations possibles avec le développement ontogénétique des Gastéropodes, des Echinodermes, et des Cténophores - § 20: Les développements pulymonodiques - §30: Lés développements cycliques ot leurs rapports possibles avec la segmentation . . pag. 323

\section{Chapitre XV.}

\section{La régénération.}

SOMMAIRE: Les conditions nécessaires de la régénération - Les limites de la régénération -- Lo nombre énormo des cellules régénératrices - Le rapport numérique entre les différentes cellules de l'organisme - Les dimensions fixes deg ôtres - L'hétérogénéité des organes - Les rapports entre les cellules et lo miliea interne - L'automatisme de la régénération - Son explication - La régénération plyysiologique et pathologique - L'origine des cellules régénératrices ot lours rapports avec los feuillets embryonnaires Cruses de l’impossibilité de la régénération - La régénération morphologique et ses conditions - Résumé . . . . . . . . pag. 340

\section{CHAPITRE XVI.}

\section{I'ontogénèse des végétaux.}

SOMMATE: Analogie de développement entre los animaux et les végétaux Parallib entro l'oeuf des animaux et l'oeuf' des plantes - Importance de la mombrano dans la segmentation de l'oeuf ot des cellules végétales - Possibilité d'uno explication de l’ontogénèse des plantes par le développement monodique - Le développement cyclique dans le développement ultériear des plantes 


\section{INTRODUCTION A LA II PARTIE}

On ne peut douter que, parmi les nombreux phénomènes biologiques, le déreloppenent le l'individu, lontnénèse, en soit un des plus intéressants et en même temps des plus difficiles a expliquer d'une manierescientifique satisfitisunte.

Lor'sque nous royons d'une cellule seule de l'organisme, de l'oeuf, dont la constitution se rérèle d'une très grande sim-

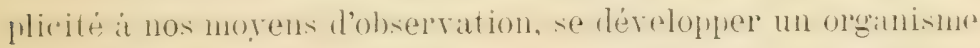
d'une complexité parfois énorme, où les organes se forment à des endroits et à des époques bien déterminés, et y acquièrent lenr constitution histologique et morphologinue, nous sommes. involontairement, je dirais mène presque forcément, amenés à supposer que cet oeuf, qui nous parait si simple, est en realité plus complexe, et que, sous l'apparence d'une certaine homónénété, il nous cache une structure morphologique ayant un rapport plus ou moins étroit arec l'organisation de l'individu qui en naîtra.

De là ces interprétations qui, même les plus modernes, sont en dernière analyse toutes préformationnistes, parce qu'elles sont basées sur la présence et sur une disposition spéciale dans l'oeuf de particules représentatives des organes futurs, soit qu'on reuille supposer ces particules disposées dans le cytoplasme de l'oeuf, soit qu'on reuille les localiser dans le noyau même.

Malheureusement les résultats des expériences sont contraires a ces sortes dinterpritations. Le déreloppement de 


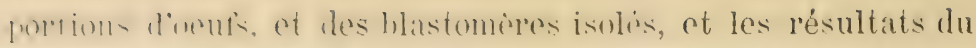

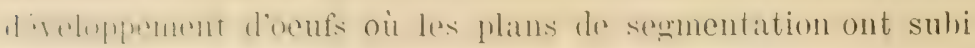
des déplacements ì la suite de conditions mécaniques artificielles, sont autant de preuves contraires ì l'hypothese d'une prélormation morphologique quelconque.

Diautre part, on ne peut douter que, par l'hypothèse de l'isotropisme de l'oeuf, l'explication scientifique des phénomines fomblamentanx de lontegénese devienne d'une difliculté qui nous paraît presque insurmontable. Et pourtant il est indiscutable que cette hypothèse, à cause des résultats des

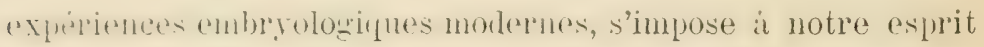
avec tant de force, que nous sommes contraints de reconnaitre que dans elle seulement peut avoir sa base toute interprétation de l'ontogénèse, rraiment scientifique.

Mais outre celles-ci ayant trait at la constitution de l'oeuf, d'autres diflicultés aussi graves se présentent lorsqu'on considère la segmentation de l'oeuf, c'est-i-dire la valeur et la nature des premiers blastomères par rapport à la nature de l'oeuf mème.

Les deux premiers blastomères sont-ils égaux entre eux et égaux à l'oeuf, ou bien en sont-ils diffẻrents?

Certes, si l'on tient compte des résultats du développement des blastomères isolés, la réponse il cette question ne peut être douteuse.

Puisque chacun des deux premier's blastomères isolés donne lieu par son déreloppement it un embryon complet, il faut arhettre nécessairement que la constitution de ces blastomères est égale à la constitution de l'oeuf. On dirait même qu'il u'! a pas d'autre conclusion possible.

Inis si, d'une part, celle-ci nous parait la seule conclusion strictement logique, l'hypothèse de l'égalité des premiers blastomeres nous met dans l'impossibilité d'expliquer les difrérenciations ultérieures et la localisation de celles-ci sans recontrir it d'autres hypothèses spéciales, ce qu'on doit tenter l'ériter dans toute interprétation vraiment scientifique. 
Expliquer les différenciations histologiques et morphologiques et leur localisation dans l'espace et dans le temps, expliquer les resultats du diveloppenent des blastomeres: isolés ou des portions d'oeufs, tout en admettant un parfait isotropisme de l'oeuf et une différence entre les blastomères et l'oeuf: roila le problème le plus arlu de l'ontogénèse, que j'espère aroir résolu scientifirnement dans cette partie de mon ourrage.

La méthode suivie dans la recherche de cette solution est d'une très grande simplicité.

Après une analrse minutiense des phénomènes fondamentaux de l'ontogénèse (chap. I); après avoir démontré que les diffrenciations histologigues ef morphologiques ne sont que les conséruences d'une diférenciation chinique des cellules (chap. II); après un examen de la constitution de l'oeuf, que je crois nexessitire pour la parfaite compréhension des phénomènes onfogénétiques (chap. III, je passe à consinlérer les bases possibles de l'ontogénèse.

Ces bases ne peurent être que les trois modes possibles de léreloppement biomoléculaire: autogénètique, homogénétique, hétérogénétique, c'est-à-dire, que les leux prenier's blastomères ou bien sont égaux entre eux et égaux á l'oeuf, ou bien sont égaux entre eux mais différents de l'oeuf, ou bien encore sont différents entre eux et diffẻrents de l'veut. Évilenment il n'y a pas d'autres cas possibles. Or, si l'on exclut les développenents autrgénétique et homogénétique, qui ne suflivent fas à notre interprétation, ainsi que je le démontre (chap. IV), il ne nous reste í consinérer que le léveloppenent hétirnénétique.

Cela étant posé, je démontre (chap. V) qu'il n'y a que deux morles possibles de déreloppements hitrogénétiques: le drreloppement polyodique et le developrement monorlique. ie premier étant écarté comme insulfisant à l'explication les phénomènes de l'ontorénèse, il ne nous reste ì considérer que le déreloppement monodique. 


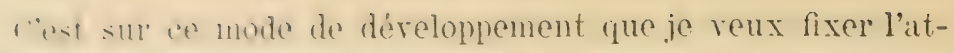

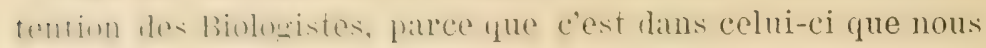
trourus de's proprites qui expliquent merreilleusement tous les principaux phénomenes ontogénétiques (chap. VI).

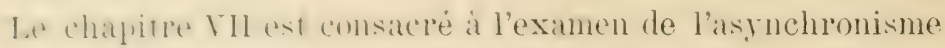
de sermentation, non comme hypothèse, mais comme conseyluence naturelle at nécessaire de mon interprétation de l'assmilation et le la cytodiérèse. Il nous explique merveillen-rament et l'une maniere absolument scientifique le rythme Wr smentation des oeufs, dont on ne savait jusqu'ici donner une explication satisfaisante.

Dans le chap. VIII, je considère la première phase de l'onturinfire, cest-it-dire la formation de l'awrégat cellulaire de sormentation en relation arec les formations morphologiques

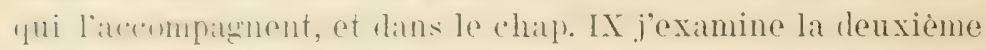
than he limtorenise of les causes de son origine, en faisant ressortir toute l'importance de la probiose dans cette sorte le pléumènes hinlogiques, et en remenant à des canses meaniqum les formations morphologiques qui caractérisent les direrses phases de l’ontogénèse.

fienule de la localication des diflipenciations histolngiques et momphologinues ast le sujet du chap. $x$, et cest dans cette

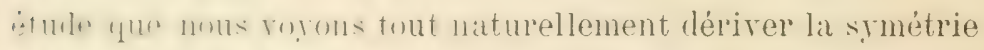
ragrunee at la -lmetrie hilaterale (chap. XI) comme simples

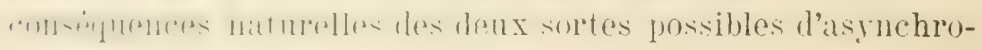
nisme rle segmentation.

Les phases ultérieures de l'ontogénèse sont étudiées dans le chap. Xill. Yous pouvons y constater facilement que mon in-

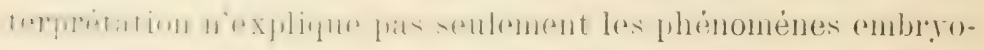
logiques, mais les autres encore qui s'accomplissent pendant tonte la vie des organismes, dont la succession constitue néanmoins leur ontogrénèse, dans la signification la plus raste de ce unot. Ia mort même y trouve une explication scientifịue, comme conséquence nécessaire du principe même de mon interprétation. 
Je recomnande surtout aux Biologistes le chap. XIlI, oi sont inoncées les solutions des problèmes de l'ontogénése. On y trumve les résultats tout-a-fatit rationnels et théoriques du developpenent de l'oeuf, de portions dre l'neut', des blastomères isolés un somuis al d'autres conlitions suéciales. C"est par lat comparaison rigureuse entre ces résultats theriques et les résultat: des expériences, que les Biolugistes pourront juger exactement de lat valeur de mon interprétation. Chacun pourrat facilement constater arec quelle srande simplicité et arec quelle précision on peut arriver, par mon interprétation, á donner une explication scientifique, naturelle et rationnelle de phénomenes hinlugiques qui nous partisialent presque inexplicables jusqu'i ce jour.

Le chap. XIT, ou j'examine les autres modes de déveloplement possibles lé-ultant d'une combinaison entre les deux moles tomlanentanx de déreloppenent hétéróénétique: le polyorlique at le monnlique, a seulement pour but de compléter l'examen de tous les moles de dérelophement possibles en nitture, atin que les Biologistes puisent roir si. dans quelques-uns de ceux-ci, on peut trouver l'explication de certains phénomènes qui nous praraissent échapper à l'interprétation générale.

Mais j'appelle toute l'attention du lecteur sur le chap. XV, traitant de lat résénération. ou l'on roit que cette faculté nerreilleuse des organismes n'est, en dernière analyse, qu'une conséquence naturelle et très simple de leur mode de développenent. C'est dans lexplication de ce phénnmène que les Biolowistes pourront encore nieux constater limportance du déreloppenent nonorlique dans l'interprétation les phénonènes de l'ontogénèse.

Je consacre enfin le dernier chapitre chap. XII a quelques considérations sur l’ontogénèse des régétaux.

J'espère que les Biologistes liront ce lirre arec toute l'attention que le sujet exige; nais quel que soir le jugenent qu’ils puissent en porter, je les prie de noublier janais le but quon doit se proposer dans cette sorte de traraus scientifiques. 
Lontoginese des organismes ne constitue pas un probleme unipue, mais autant de prohlemes qu'il y a d'epereces d'orgatnismes, et je dirai meme antant de problemes qu'il y a d'individus.

Il ne s'agit donc pas de donner une solution de tous ces problemes, parce que celat serait impossible, vu notre connaissance trop impartite des facteurs dont elle dépend. Il s'agit seulement de trourer le primeipe fondamental qui régit le phénmmène ontogénètique. On ne peut éridemment prétendre davantage dans une interprétation de l'ontogénèse.

Jessaielai te n'expliquer plus clitirement par un exemple, lequel, bien qu'inparfait, servira néammoins a faire comprendre assez exactement ce que je viens de dire.

L'untogénése, l'ensemble de toutes les transformations qu'un organisme subit du commencement de son déreloppement jusqu’à la mort, est sans aucun doute un phénomène météorique et reut ètre comparé it d'autres fhénomènes de cette nature, par exemple, au parçour's d'une rivière.

Or, si l'on roulait déterminer d'avance ce parcours dans tous ses détails, il s'agrirait lit évidemment de la solution d"un Inohlene le la plus grande difliculté au proint de rue pratique; car cette solution exigerait la connaissance de plusieurs factenr: vitesse et densite de l'ean, inclinaison et constitution

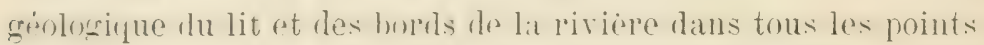
de son parcours, résistance des matéliaux qui les constituent, accidentalité du sol etc. On comprénd facilement que la connaissance exacte de tous ces facteurs est tellement difficile gur la sulution du problène devient, daus la plupart des cas, impossible. Et pourtant, rien n'est plus possible et. je dirais

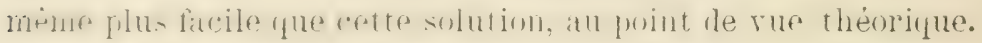
Mais d'où dérive-t-elle, cette possibilité? De la connaissance du principe fondamental régissant la chute des corps, et, par suite, de l'eau: la gravité.

Mais sans ce principe, la solution du probleme serait absolument impossible, quand mème on possèderait une connaissance 
absolument exacte de tous les facteurs mentionnés. De sorte que. au point le rue thénrique, la prublene énnncé est et doit itre consideré comme parfititement léwoln, et lit solution est le résultat de la connaissance du principe fondamental.

Il en est de même de l'ontogénèse.

Ce n'est pas la connaissance des facteurs secondaires qui est nécessaire pour la solution de cet important problème

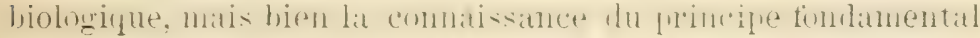

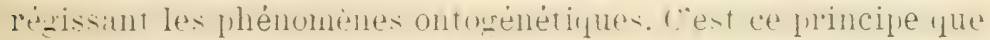
les Biologistes doirent, rechercher, et c'est ce principe que j'espère avoir trouvé dans le déreloppement monodique.

De même que la gravité est le principe fondamental unique régissant le parcour's de toutes les rivières, et que les facteurs mentionnés ne sont que d'importance secondaire, parce qu'ils peuvent changer pour chaque ririère tandis que le principe fondamental ne change point; de même, il peut exister pour lontowenes un princine findinnental uninne et innulable, bien que les facteur's secondaires soient au contraire extraordinairement rariables.

Et c'est précisément ì ces facteur's qu'on doit l'énorme ra-

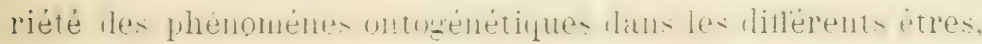
tout comme dans l'exemple cité, c'est aux facteurs secondaires mentionnés qu'est due la grande rariété des parcours des différentes ririères, bien que le principe fondamental soit pour toutes absolument le même.

La solution de chaque problème ontogrénétique est donc subordonnée à la connaissance de ces facteur's; mais la solution de tous les problimes, lans leur enselnble, est arant tout depentante de la connaissance du frincige fomlannental.

Les Biologistes pourront, par une analyse rigoureuse, juger

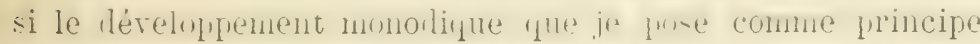

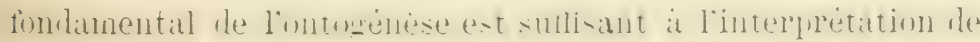
tous les problemes. Ils constilteront que ce principe est llune simplicité extramblinare, comme tuus les principes les phe nonuéres de la natures: et pourtant, cette simplicité nume, ce 


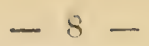

qui de prime aborel parait absurte, amine tout naturellement ot néresarement it une complexité tellement énorme, que monle esprit segare dans la consilération de tous les phénomènes qui peurent en dériver.

The hati encore remarquer yu'en suirant rigoureusement la méthode qui est, je l'espère, la base et la force de toute ma théorie des phénomènes de la vie, j'ai exclu absolument de mon interpretation de l'ontogence toute hypothese spéciale, en me hisint uniquement et exclusirement sur les proprietes générales des corps bruts, que les Biologistes ne sauraient certainement refuser aux corps rivants.

D'ailleurs, mon interprétation de l'ontogénèse peut heureusement subir un contrôle rigoureux de la part de la Biologie expérimentale. J'arrive par mon principe, ainsi que le lecteur pourra maintes fois le constater, à des conclusions, et surtout à des solutions de certains problèmes d'une telle précision, que l'expérience pourra facilement constater si mes résultats thérniques (onrespondent parfitement aux résultats experimentaux.

crost ce contrôle que je désire et que j’espire pour le triomplie de la vérité. 


\section{Chapitike I.}

\section{Les phénomènes fondamentaux de l'ontogénèse.}

SOMMAIRE: Les phénomènes fondamentaux de l'ontogénèse - La prolifération cellula:re - La différenciatiou histologique - La différenciation morpholog que - La localisation des différenciations - Concomitance de ces phénumènes Examen de la prolifération cellulaire - Examen de la différenciation histologique - Histogénése glandulaire - Histogénèse des cellules adipeuses, des Érythrocytes et des cellules cornées - Histogénèse des tissus conjonctifs et du tissu mus zulaire - Histogénèze des celtules verveuses - Examen de la différenciation morphologique - Importance capitale du plissement des feuillets germinatifs et importance secondaire du cytotrop:sme dans la différenciation morphologique - Examen de la losalisation des différenciations - Localisation de la prolifération cellulaire et des différenciations histolo. gique ot morphologique - Résumé.

L'ensemble de toutes les trinsformations successives graduelles, par lesquelles du germe se développe l'organisme, constitue l'ontogénèse.

Tous les êtres rirants, même les plus simples, ont donc leur ontogénèse; cependant, dans cette partie de mon travail, je me bolnerai a traiter exclusirement de l'ontogénèse des animaux pluricellulaires.

En quoi consistent ces transformations ontogénétiques? Nous n'avons qu'ia comparer la nature et la constitution du germe avec la structure et les caractères de l'organisme qui en dérire, et nous rerrons, dans les différentes propriétés de l'ètre à ces deux stades de sa vie, quels sont les phénomènes caractérisant l'ontogénèse.

Le germe des animaux est formé d'une seule cellule: la cellule oeuf. L'organisme adulte ou presque adulte est, au contraire, constitué de plusieurs cellules. Pendant l'ontogénèse, 
il y a dome anowisement du nombre des collules, cest-it-dire: "prolifiration cellulaive ». Toili un des phénomènes fondamentaux de l'ontogénèse.

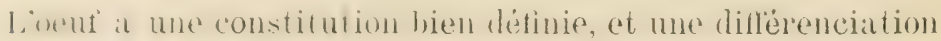
histologique à lui. Le germe, constitué d'une seule cellule, ne presente donce qu'une sante dinlérenciation. L'organisme issu du germe est, au contraire, formé de plusieurs cellules home les differenciations histologiques sont tres différentes. P'embant l'onfogénese, il y a donc une transtormation bien sensible de la constitution intime des cellules, c'est-à-dire une

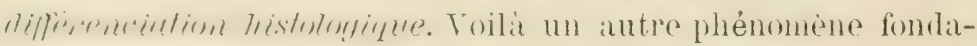
mental de l'ontogénèse.

Les nombreuses cellules constituant l'organisme ont entre elles une disposition qui donne lieu à certains agrégats cellukaires généralenent bien déterninés dans leur forme et dans leurs dimensions: les organes. Il est done ẻvident que, pendant l'ontonénirse, les niflirentes cellules issues de l'oeuf so groupent entre alles l'une manibre spéciale, telle que l'exige la forme

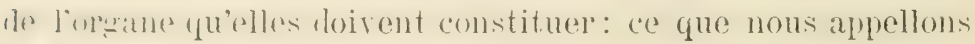

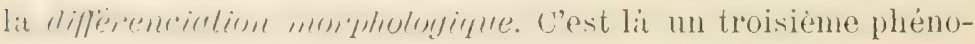
mène foridamental de l'ontogénèse (1).

Bien que certains Biologistes se soient efforcés de trouver dans l'oeuf' une disposition de ses substances préludant en quelque sorte à la disposition que les diverses parties présen-

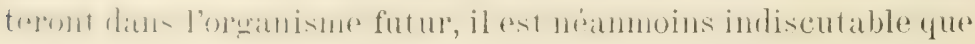
ces eflorts ll'ont pas abouti jusqu'ici à des résultats viaiment

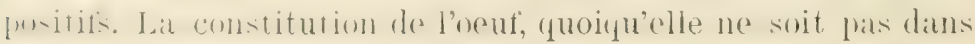

(1) On pourrait ajouter encore une antre difiérenciation que j'appellerai cytomorphologique, c'est-à-dire la difórenciation de la forme des collules aux phases diverses du développement, par rapport à lour diffórenciation histologique, à l'exclusion, bien ontendu, des changements de forme qu'elles penvent affecter sous les actions mécanirnes de pression on d'adhésion. Mais comme cetto différenciation cytomorplologique est étroitement lí6o is la rlifiérenciation histologique, ainsi que nous le pouvons constater toujours, jo la comprends implicitement dans cetto derniere. 
tous les cas absolument homogène, ne nous révèle rien sur l'arrangement des futur's organes. Lorgunisme, au contraire, nous présente dans la disposition de ses parties une symétrie et une polarité bien marquées, ce qui nous indique que, pendant l'ontogénèse, les différenciations histologiques et morphologiques ne se produisent pas seulenent, mais qu'elles se localisent aussi dans des endroits déterminés. C'est ce qu'on appelle la "localisation des différenciations ". Voilà donc un quatrième phénomène fondamental de l'ontogénèse,

Le complexe processus ontogénétique est donc constitué des quatre phénomènes fondinnentaux suivants: $1^{\circ}$ ) la prolifération cellulaire; 20) la différenciation histolónique: 30) la différenciation morphologique; t") la lucalisition les diférenciations. Il est, par suite, évident que toute interprétation de l'ontogénèse devra nous expliquer pourquoi et comment les phénomènes ontogénétiques peuvent s'accomplir.

Cependant, il faut se rappeler toujour's que ces phénomènes ne sont pas inlépendints les uns des autres, mais qu'ils sont, au contraire, réliés entre eux d'une manière très intime. Ils s'accomplissent simultanément, c'est-î-dire qu'ils sont tous les quatre concomitants.

kn effet, une différenciation histologique, quelle qu'elle soit, ne se manifeste pas á un moment quelconque du développement, mais à une phase donnée de celui-ci, c'est-à-dire lorsque l'embryon est formé d’un certain nombre de cellules. La diffërenciation histologique est donc etroitement dipendante de la prolifération cellulaire.

De mème, la forme des diver's orranes n'est pas indifférente, mais elle est très intimement connexe à la différenciation histologique qui les caractérise. Le foie ne prend jamais la forme du cerveau, ni le cerveau la forme des poumons ou des reins. Cest-it-dire que les cellules destinies at subir telle ditfërenciation histologique, se groupent aussi d'une manière particulière correspondant a cette difrërenciation, et conduisant, par' suite, à la formation d'un organe de forme déter- 
mincis. Ia dillipenciation morpholupique est done ètroitement liée ì la diflérenciation histologique.

De mème aussi, la disposition que les organes présentent

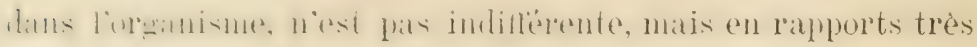
étroits arec leur nature histologique et morphologique. Le foie et les poumons ne se forment jamais dans la cavité cranienne; le cerreau ne se loge jamais dans la carité abdominale; et nous ne royons pas qu'un des yeux se forme, par exemple, dans la tête et l'autre sur le thorax. Tous les organes ont une position fixée dans l'organisme et cette position ent en relation aree leur constitution histologique et leur forme. La localisation des différenciations est donc liée aux differenciations histologiques et morphologiques.

On pourrait donc expliquer parfaitement comment s'acconplit lat proliération cellulare, comment se fait la dirérenriation histolngipue et cytomorpholngique, comment se forment les organes, comment enfin ils se localisent, que cela ne suflirait pas encore à la parfaite interprétation de l'ontogénèse.

Ce qu'il faut absolument démontrer, c'est la cause et en

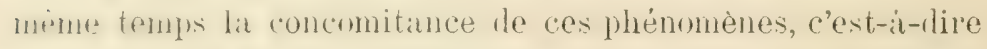
lar qurlles forces la prolifüration cellulaire, et les différen"bitions histolugique, morphohoique et lat localisation de ces

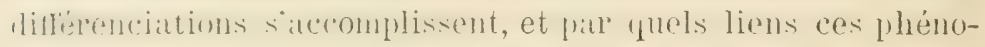
mènes sont en relation entre eux.

On roit donc que les prohlèmes ì résoudre sont beaucoup plus graves qu'ils ne le paraissent au premier abord. Mais pour arrivel à une interprétation exacte et sumisante des filemonimes ontormetiques, il faut arant tout les analyser minutirusmuent, afin d'en connatre la nature intime dans tous ses détails. C'est ce que nous ferons dans ce chapitre.

Commençons par' l'examen de la prolifération cellulaire.

Ce phénomène - nous le savons - n'est que la conséquence directe de la division cellulaire, de la cytodiérèse. Mais, comme l'assimilation est la condition indispensable de 
la crtorièrèse (1), on comprend futement que la prolifération cellulaire ne peut aroir lieu sans l'assimilation.

D'autre part, comme l'assimilation est un phénomène chimique, il exigera, pour son accomplissement, les conditions nécessaires it tout phémonène chimique: c'est-it-lire: l") une substance capable d'assimiler; $2^{\circ}$ ) une substance ou des substances aptes à itre assinilées; ;3) des conditions physiques convenables aux phénomènes de l'assimilation.

Or, toutes ces conditions se trourent réalisées en partie dans l'oeuf mème, en partie dans le milieu ambiant où l'oeuf se développe.

En effet, comme nous le verrons bientòt, l'oeuf contient en lui-mème deux substances: le bioplasma et le deutoplasma. Le bioplasma est l'ensemble de toutes les biomolêcules et des biomores constituant la substance virante orulaire, qui, par conséquent, est capable d'assimiler. Le deutoplasma est un mélange de substances brutes particulières, incapables d'assimiler, mais aptes à fournir au bioplasma les éléments nécessaires pour son assimilation.

Quant aux conditions physiques, l'humidité, la lumière et surtout la chaleur, elles sont fournies par le milieu ambiant, extérieur à l'oeuf.

La prolifération cellulaire ne présente done pas de diflicultés pour son explication.

Il n'en est pas de mène de la ditfërenciation histologique. Les idées des Bioloristes sur la nature réritable de ce phénomène ne sont pas encore aujourd'hui bien exactes, et c'est là une des causes principales qui s'opposent it la ju-te interprétation de l'hérédité des caractères dans les organismes issus de la fécondation, et dans les hybrides. Nous verrons, dans une autre partie de ce travail, que la connaissance la plus parfaite de ce phẹnomène est d'une nécessité absolue, et nous pourrons alors en apprécier exactement la valeur.

(1) Giglio-Tos E. - Les Problèmes de la Fie. 1e Partie. Chap. VI. 1900. 
L'étude des phénomènes de l'histogrénèse est, sans aucun donte, très dillicile. Aussi, les processus très intimes qui se passent à l'intérieur de la cellule lor's de sa difrérenciation histologique ne sont pas encore bien connus. Cependant nous verrons que les resultats positifs qu'on posisele aujourd'hui, sont sullisints pour nous donner une idee assery exacte de la réritable nature de l'histogénèse.

Vous eximinerons ici quelyues-uns des principaux processus histogénétiques, tels que M. Inval les expose tress clairenent dans son traité athistologie (1): et, comme l'histogénèse grlandulaire est lit plus simple, lit plus typique et celle qu'on doit considérer comme la base de toutes les autres, jo commencerai par celle-ci.

Histogénèse glandulaire. - L'histoginese des cellules caliciformes de l’intestin peut ritre choisie, grice a sa simplicité, comme týpe de cette espèce d'histogénèse.

Ces cellules ont la forme d'un verre a boire dont les parois sont formées par un protoplasma granuleux plus ou moins abondant à la partie inférieure. C'est dans cette partie que se trouve refoulé le noyau, tandis que la cavité de la cellule caliciforme est remplie par une masse claire, homogène, présentant toutes les réactions du mucus.

Or, c'est un fait désormais bien démoniré que cette substance nét due qu’a la sécrétion du protuplasma des cellules cilliciformes. Il y a donc lieu de distinguer dins ces cellules deux substances: $1^{\circ}$ ) le protoplasma granuleux constituant

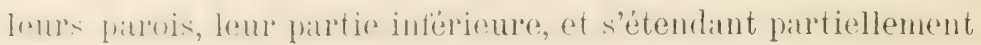
a l'intérieur de la cavité, oì il ne forme qu'un réseau dont les mailles circonscrivent des vacuoles: c'est la partie rraiment rirante de cette espèce de cellules, c'est en somme leur bioplasma; $\left.2^{0}\right)$ le mucus, c'est-i-dire une substance qui n'est pas virante, qui n'est donc pas a bioplasma, et dont la nature spéciale, caractérisant cette sorte d'éléments histolo-

(1) Duval M. - P'ócis d'llistologie. Paris, 1897. 
gripues, tient it la constitution chinique de lit substance rivante: c'est le produit de sécrétion du bioplasma.

Yous pourons donc conclure: In diffisencintion histrologique des cellules caliciformes consiste dans ce fail, que le bioplasma de ces cellules, issues de la cellule-oeuf primitive, a acquis une constitution chimique capable de donner, comme piroduit de sa sécrétion, la substance chimique spéciale que nous appellons le mucus.

Nous pourrions examiner l'histogénèse d'autres cellules glamdulaires quelles quelles soient, que nous en obtiendrions le même r'ésultat.

Il est d'ailleurs érident que les substances sécrétées et la forme des cellules pourront être très différentes, et que, en rapport avec la nature des produits de sécrétion, le mode même d'élimination de ceux-ci variera notablement. C'est ainsi, par exemple, que si les substances sécrétées sont liquides, elles pourront diffuser plus ou moins facilement à l'extérieur de la cellule, en en traversant par osmose la meminane. Cela dipendra naturellement de la nature les liquides: sécrétés et de la nature de la membrane cellulaire, d'après les lois réglant les phénomènes osmotiques.

si ces liquides sont ficilement diffusibles it trarers la menbrane cellulaire, ils en seront éliminés aussitôt qu'ils sont produits, et leur présence dans la cellule, peut être, ne sera pas facile a constater. Mais si, au contraire, ils diffusent difficilement, ils s'accumuleront alors dans la cellule et en augmenteront le volume.

Enfin, si la substance sécrétée est solide ou presque solide, ainsi que nous le pouvons roir dans les cellules des glandes sébacées, ou de la glande du noir des Céphalopodes, elle ne pourra alsolument lifruser; elle s'accunulera it lintérieur do la cellule jusqu'à la faire crerer, et de cette manière aura lieu son élimination.

Cependant, tous ces phénomènes secondaires, qui peuvent rarier extraordinairement, ne sont que des modalités sans 
importance pour nos études. Ce qu'il importe en tous cas de constater, c'est que le phénomène de la difrérenciation glandulaire consiste toujours dans la faculte acquise par le bioplasma de cerlaines cellutes de serreler des substances spéciales.

II istogénèse des cellules adipenses. - L'eximen de la for mation de la graisse dans les cellules adipenses nous conduit it des conclusions analogues.

En effet, "la cellule adipeuse est, au début, une simple masse de protoplasma arec un noyau: ce protoplasma, avec les matériaux que lui livre le milieu intérieur (sang, lymphe), élabore de la graisse qui apparaît d'abord sous forme de fines gouttelettes éparses dans le corps cellulaire: ces gouttelettes grossissent, viennent en contact les unes des autres et se fusionnent pour former la grosse goutte de graisse qui distend la carité centrale de la cellule adipeuse: en même temps, mais tardivement en général, ce protoplasma s'est sécrété une membrane cellulaire » (1).

Nous pourons done comparer parfaitement la cellule adipeuse à une cellule glandulaire dont la substance sécrétée, en raison de l'absence de diffusibilité, s'accumule á son intérieur, et nous conclurons que la différenciation adipense consiste dans la facullé acquise par le bioplasma de cerlaines cellules de sécréler de la graisse.

Histogénèse des érythrocytes. - Nous sarons que les érythrocytes ou corpuscules rouges du sang des Vertébrés sont des. cellules contenant l'hémoglobine. Quelle que soit leur structure, quelle que soit leur origine, il est indiscutable que cette espèce de cellules, à un starle plus ou moins reculé de leur évolution, ne possédait pas encore de l'hémogglobine.

Cependant, comme cette hèmoglobine ne se tronve pas dans le plasma sanguin dans lequel baignent les érythrocytes, on ne peut supposer que l'hémoglobine contenue dans ceux-ci

(1) Duval M. - Précis d'llistologie. Paris, 1897, p. 79. 
soit due à la pénétration de cette substance de l'extérieur à l'intérieur d'eux. Fit, si nous excluons cette origine, nous ne pouvons expliquer la présence de l'hémoglobine dans les érythrocytes, qu'en la supposant un produit de sécrétion de leur bioplasma.

Nous pourons donc conclure, dans ce cas aussi, que la dif-

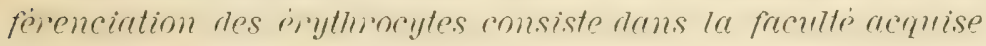
par leur bioplasma de sécréter l'hémoglobine.

Histogénèse des cellules cornées. - Un phénonène très fréquent dans les cellules de la peau des rertébrés, c'est leur' transformation en cellules cornées. Toici comment elle s'accomplit.

Les cellules de la couche de Malpighi donnent lieu, par prolifération, à d'autres cellules qui passent dans les couches supérieures. Tant qu'elles se trourent dans la zone malpighienne, le corps de cellules n'est constitué que de bioplasma, c'est-à-dire qu'on ne voit dans leur intérieur pas d'autres substances que le norau et les gramulations très fines du cytoplasma. Mais, dés qu'elles arrivent, par des déplacements successifs, dans le stratum gramulosum, on roit que leur bioplasma est semé de gouttes, parfois rolumineuses, d'une substance liquide, d'aspect huileux, à laquelle Ranvier donna le nom d'éléidine.

Dans le stratum lucidum, qui est une couche superposée à la précédente, les cellules sont plus aplaties et leurs noyaux sont dejja très sensiblement atrophiés: ces cellules renferment également de l'éléidine, non plus sous la forme de gouttes ou granulations, mais a l'état de masses rolumineuses. «Il semble donc que la formation de l'éléidine se fasse d'une manière graduelle, comme une élaboration, commençant dans les couches profondes pour atteindre son maximum dans les couches superficielles du stralum granulosum et enfin infiltrer tout le stratum lucidum (1) ».

(1) Duval M. - Loc. cit., p. 233. 
Entin, lans les couches plus superficielles qui forment, par

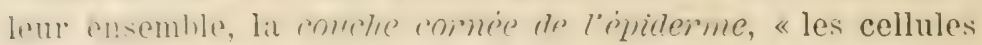
sont plates, riduites, chacune, à l'état d'une lame desséchee: en etliet, ces cellules ne sont plus formées par du protoplasma Eranuleux, mais par une substance élaborée par le protoflasma, la livoline ou substance cornée, accumulée surtout dans les couches périphériques de la cellule (1) ». Très prohahlement cette liératine n'est due qu'à la transformation ultérieure le l'eléiline élaborée aupararant par les mêmes. relluies, cest-it-dire que l'éléidine représenterait un stade de la formation de la kératine.

Yous royons donc, ici encore, que In différembiation histo-

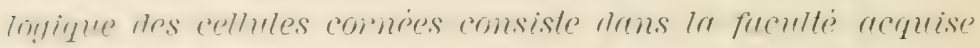
par leur bioplasma de sécréter de l'éléidine, de la kératine ou d'autres substances analogues.

Histogénèse des tissus conjonctif's. - L'examen de l'origine des diffépents tissus conjonctifs nous porte à des conclusions semblables a celles que nous renoms d'établir four les autres différenciations.

On sait en effet que le tissu conjonctif mquenx dérive des rellules du mésenchrme ou tisu conjonctif embryonnaire, lesquelles deriennent fixes, sanastomnsent par leurs prolongranents, et élahorent, exsulent et accumulent dans les espaces on mailles interposés entre elles et circonscrites par ces fmonstuents, une substance particuliere, transparente, hyaline, semi-liquide, formée presque en totalité de mucine.

Pour ce qui regarde l'origine des fibrilles du tissu conimnlif fil, illni,e, on sait que les anciennes interpretations de SCHWANy et de IIENi.E, d'après lesquelles ces fibrilles dériveraient de la transformation totale ou partielle de la sub-tank l,mpla-mat ique mene des cellules, sont désommais abandonnées.

I)'autre part, ruoique les opinions de VIRCHow, d'après les-

(1) DUYaL M. - Loo, cit., p. 226. 
quelles les fibrilles du tissu conjonctif seraient sans rapport grénétique arec ses cellules, n'atient fras encore été démontrées tont a fait erronées, on nr peut nier toutefois que les résultats des recherches les plus récentes ne plaident pas en fareur le cette interprétation. Ils sont, au contraire, suffisants pour nous démontrer que les fibrilles du conjons if sont un prouluit de l'élaboration du bioplasma de ses cellules.

La question controrersée, à saroir, si ces fibrilles dérirent de la seule partie exoplasmique de ces cellules, ou bien si lenroplasma intervisnt, hi aussi, dans cette élabolation, n’a pas d'importance pour notre sujet. Ce qu'il importe surtout d'établir, c'est que les fibrilles du conjonctif ne sont pas le resultat dune transfonation totale on partielle de la partie virante des cellules conjonctives, de leur bioplasma, mais qu'elles en sont un produit de sécrétion direct ou indirect.

L'étude de l'origine des fibres élastiques nous permet d'arriver à des conclusions analogues.

* Il est à peine besoin de rappeler que HExle arait émis l'hrpothese que chaque fibrille elastique résulterait de l'allongement du noyau d'une cellule après que le corps cellulaire de celle-ci se serait en totalité transformé en fibrilles conjonctires, dou la nom de fimes mu nomm, fitmes mulenires, donné aux fibus élastiques. Mais aucune obserration ne confirna cette manière de roir et toutes les recherches aboutirent ¿́ fuire considérer. au contraire. la fibre élastique comme jurduite par la transformation de la totalité d'une cellule. Les cellules du rissu conjunctif s'anastonnosent en résean frar leurs prolungements. Cesont ces polongements qui diabort se transformeraient en substance élatique, puis le corps cellulate

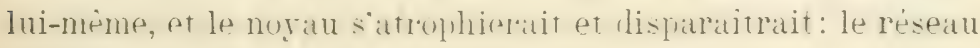
cellulaire deriendrait ainsi réseau élastique ».

"Cependant, de- 1817, Mreller arait constaté que, dans les cartilages réticulés, les fibres élastiques apparaissent dans la substance fondamentale ou intercellulitre. mimitirement hraline, et ne présentent aucun lien génétique direct arec les 
rellules de ce tissu (cellules cartilaginenses); et Foembirer, ayint suri arec soin lem mole d'apparition, les rit se produire frar juxtaposition en série linéarire de corpuscules ou molécules élastiques ».

"Ce mole de prouluction, dams la substance intercellulaire, sins interveution linecte des cellules, a èté confirme par un frami numble de recherches et spécialement par celles de RANTIER •

* Il est donc incontestable que les fibres élastiques se produisent lans lat sulstance fondamentale on intercellulaire. Iats, de mine que pour les fibrilles conjonctives, cela ne reut fas ilire que les cellules restent étrangères a cette production: elles la luroroguent, au noins par me action indirecte, puisque lit nutrition de cette substance, ses transformations sont sous la dépendance de ces cellules. Et ici encore, nous derons penser que cette action, indirecte dans les cas sus-indiqués, peut aussi derenir directe dans d'autres cas, et qu'alor's la fihre élastique sera une émanation on meme une élabolation immédiate du protoplasma » (1).

(anant it l'origine des différents tissus cartilaginenx, il n'y a fric de doute que leur substance fondamentale et celle qui firmur los caprules dans lesquelles les cellules cartilagineuses -mut renfinpuées, sont dues it la sécrétion de ces cellules mênes.

Tous pourons donc arriver à cette conclusion, que, les dif-

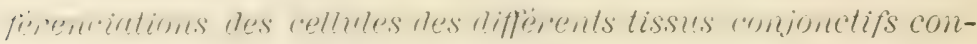

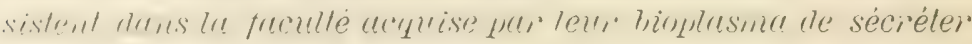
cerlaines substances particulières caractérisant ces divers lissies.

Histogénèse musculaire. - L'éturle de lorigine des fibres mun-rulains rst, elle anssi, tres instructive an point de vue

(1) Duval M. - Loc. eit., p. 358-361. J'ajonte ici que ces id6́es sur l'origine des fibres blastirues trouvent uno confirmation dans les résultats olteuns par Err. C'. C. HANSEx ot exposés dans son travail: Ueber die Genese einiger Bindegenebsgundsubstanzen. Anat. Anz. Bel. XVI, 1899, p. 417-438. 
de l'importance qu'a la sécrétion dans la différenciation histologique.

Les cellules destinées à derenir fibres musculaires sont les imyoblastes. Ceux-ci, tous d'abord de forme sphérique et pourvus d'un seul noyau s'allongent de plus en flus, tandis que leur noyau primitif unique, par des uirisions répétées, donne origine à plusieur's noraux qui se disposent suivant l'axe de la cellule. A un certain moment de leur érolution, e ces myoblastes commencent à présenter les détails caractéristiques de la future fibre striée: dans la couche périphérique de leur protoplasma apparaît le la substance musculaire, c'est-à-dire des fibrilles transversalement striées. Alors le myoblaste a l'arjact d'un tuhur, dont la paroi est formée d'une couche de fibrilles striées, et dont le centre est occupé par du protoplasma granuleux arec des noyaux. Ces fibrilles striées d'emblér, aplatracsut dans la frotoplasma périphérique comme un cristal apparait, présentant d'emblée ses caracteres, dans une dissolution capable de lui donner naissance; elles sont wne élaboration toute particulière, encore mal connue, du protoplasma; mais elles ne sont pas le résultat de la transformation d'une cellule ou d'un noyau; elles sont dans leur ensemble une elaboration endoplassinque du myoblaste. Les noyaux placés dans l'axe continuent à se unultiplier par cartrocinèse; le myoblaste continue ainsi à s'allonger et ¿ s'épaissir. En mène temps, son écorce de fibrilles striées est derenue plus puissante, par formation incessante de nourelles fibrilles » (1).

Dans ce cas aussi, nous pouvons done conclure que la dif-

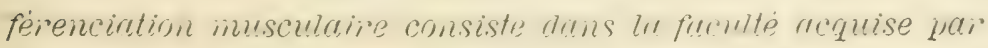
le bimplasur! de revtaines cellules de secreter une substrince parliculière contractile.

Histogénèse des cellules nerveuses. - Les éléments cellulaires qui donnent origine atux cellules nerreuses sont les

(1) DưaL M. - Loc. cit., p. 544. 


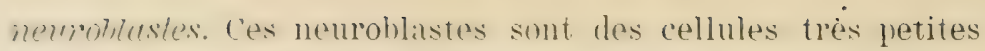

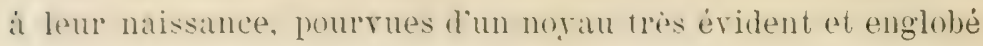
dans me masse de cytoplasma lelatirement peu abondante.

On ne sait pas encore d'une manière précise par quel processus ces nemoblastes deviennent les cellules nerveuses arec leurs garticularités et luolongenents caratéristigues. On comnat senlement yulils se transforment en cellules nerreuses par laangmentation en rolume de leur noyau, et surtout de leme protoplasma, ot par le firit que celui-ci dmet des prolongements.

Cependant, si nous jureons par antlorio avec les autres différenciations, je crois que nous pourous retenir avec une grande raisemblance que les nombrenses fibrilles qui constituent en partie le corps des cellules nerveuses et qui se poursuirent dans lems's prolongements, et le prolongement cylindre-ixile mine sunt formés de substances sécrétées par le bioplasma des neuroblastes, tout comme les fibrilles contractiles des fibres musculaires le sont du bioplasma des myoblastes.

Je crois done que nous pourons, dans ce cas aussi, arriver a cette conclusion: que la differenciation neremse consiste

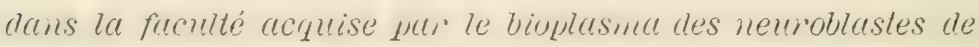
sécrétè cerlaines substances parliculières junissant de la propriété caractéristique de la sensibilité.

Je pourais mentionner encole d'autres exemples d'histo-

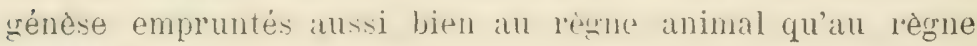
viuetal. Ce n'est que pour plus de hriereté yue je ne le fais pas.

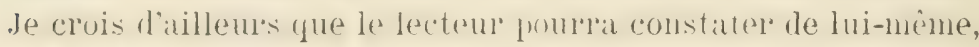
en analysint attentivenent les liflèrents processus histogénétiunes, qu'ils présentent toujours me grande analogie arec ceux que je viens d'exposer.

Les exemples mentionnés suffiront, je l'espère, pour nous

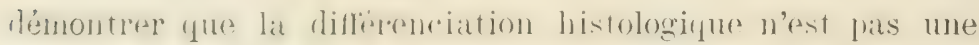
tratn-formation totale on foutiollo du bioplatsma des cellules

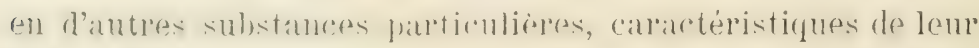

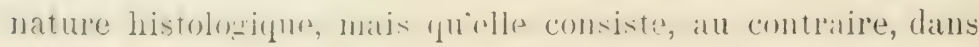


une réritable sícrétion de ces substances de la part du bioplasma. La différenciation histologique n'est, en somme, qu'une simple sécrétion bioplasmatique.

Passons maintenant à la diffẻrenciation morphologique.

Tous n'arons qu'i exaniner attentivenent les phénonènes embryologiques fondamentaux les plus communs, et nous rerrons en quoi consiste cette différenciation, et de quelle manière elle s'accomplit.

PAxDER, un des fonlateur's de la théorie des feuillets, s'était dẹja expriné très clairement à ce sujet: « La membrane germinative - dit-il - donne naissance à la paroi du corps et aux riscères de l'animal par le simple mécanisme du plissement. Un filanent délicat se montre dans le germe et refrésente la moulle épinière. À peine ce filament est-il formé, qu'apparaissent atu-dessus de lui les prentiers replis, constituant la première ébauche de la paroi du corps. Puis le germe se plisse de noureau; mais ces noureaux replis se dirigent en sens inverse des premiers et donnent lieu à la formation de la carité abdoninale et de la carité thoracique arec leur contenu. Enfin le germe se plisse une troisieme fois pour produire les enveloppes du foetus » (1).

Après PAxDer, ce fut Lotze qui le premier reprit l'étude du mécanisme de la furmation de l'embryon. Il considère « l'accroissement inégal 》 ou «la régétation inégale * comme la cause des transformations subies par l'embryon dans le cours le son déreloppement. Ces transformations qui u'apparaissent, d'aplès Lotze, que comne des deplacenents, des évaginations, des inraginations ou des extensions de membranes, seraient en réalité produites par traction ou par pression mécanique.

Si nous passons à des travaux plus récents, nous arrivons aux études de Hrs (2) sur le mécanisme phrsiolorique du dé-

(1) Pander. - Entwickelungsgeschiohte des Küchels. Oken's Isis, 1818, Tom. I, p. 512-524.

(2) His IV. - Unsere Körperform und das physiologisohe Problem ihrer Entstehung. Loipzig, 1875. 
veloppement. IIs s'est occupé de cette question l'une façon plus actire que tous ses prédécesseur.s. Il a de noureau appuyé énergiquement sur le role important que joue le processus du plissement dians la formation de l'embryon, et l'a même posé comme base de son interprétation de l'ontogénèse (l).

Que la diflérenciation morphologique repose, en toute première ligne, sur un processus de plissement des lamelles épithéliales, c'est ce que les frères Hertwig ont cherché à démontrer plus complètement encore que leurs prédécesseurs, en s'appuyant sur de nombreux matériaux de recherches, et c'est d'ailleurs ce que les plus récents résultats des observations embryologiques ont pleinement confirmé.

Cependant, dans le cours de ces dernières années, un nouveau facteur, le cytotionisme, c'est-i-dire la faculté qu'ont les cellules embryonnaires de s'attirer réciproruement, a été introduit par Rocx, comme ficteur jount un ròle typique formatif dans le développement de l'individu (2).

Je ne reux pas nier d'une manière absolue l'importance de ce noureau ficteur; toutefois je dois faire remarquer que le cytotropisme ne peut pas s'exercer a de granles distances, et que d'autre part, dans les conditions normales, il ne peut avoir jeu qu'entre les cellules jouissant d'une certaine mobilité, telles que la plupart des cellules du mésenchyme. Or, nous savons que, si le mésenchyme joue un róle qui n’est pas négligeable dans la différenciation morphologique, il est néanmoins indiscutable que le roble principil et essentiel est en tout cas réservé aux cellules dérivées des feuillets épithéliaux.

L'examen de la formation des divers organes nous démontre tres clairement que la forme de ceux-ci est essentiellement dépendante du morle de prolifération des cellules dont ils dé-

(1) Hentwig O. - Traité d'Embryologio de l'Hommo et dus Verlébrés, trad, par C. Julis. Paris, 1900, P. 206.

(2) Roux IV. - Ueber den «Cytotropismus》 der Furchungszellon des Gras. frosches (Rona fusct). Arch, f. Entwickelungsmech., I Bd., 1895. 
rivent et des circonstances nécaniques qui accompagnent leur formation. Le cytotropisme pourla lonc bien attirer rers ces organes quelques-mes des collulus du mesenchyme, mais je ne crois pas que celles-ci soient capables de modifier notablement la forme typique de l'organe.

Sans refuser toute importance au cytotropisme, nous pourons donc conclure que la differenciation monphologique est essentiellement le iésultat de plisscritents, d'écaginations et d'invaginations qui ont leni origine duils la piolifération cellulaire et dans les circonstances accaimurngiant celle-ci.

Quant à la localisation des dilférenciations, tous les Biologistes qui ont comnaissance des plıénomènes ontogénétiques savent en quoi elle consiste.

Les plissements, les évaginations et les invaginations cidessus mentionnées, qui donnent lieu ia la différenciation morphologique, ne s'étendent pas à tout l'embryon ni ne se produisent çia et lì indiflëremment: elles se localisent au contraire dans certains entroits de l'enbryon, suirant l'espèce de celui-ci.

C'est sur ce phénomène que PANDER arait déjà rappelé vivement l'attention des Biologistes.

"Quand je parle - dit-il - de plissements de membranes, je n'entenrs nullement dire patr lit yu'il s'agisise de membranes inaninées, dont des plis fornés mécaniquement s'étendraient fatalement sur toute la surface, sans se limiter à une région déterminée. Les replis engendrés par la métanorphose de la membrane germinative sont l'origine organique et se forment en des points bien déterminés, soit par accroissement des spherules (cellules) qui y existaient aupararant, soit par tormation de nourelles sphérules, et cela, sans qu'il en résulte des modifications dans les autres parties de la membrane germinative ».

Le phénomène caractéristique de la localisation des plissements ne doit jamais ìre oublite laus l'étule de l'ontogénése. Je me rallie donc parfaitement a O. Hertwig, lorsque, en admettant un grand nombre des irlées émises par Hrs sur le 
méandisme de lat dormation nes organes, il ne peut néammoins alfureurel sit miniere de voir sur certains points importants. Lorspute pas extmple. His reut ranener le mécanisme de la formation le lembryon al simple probleme des changements de limme lome lame élastique yui serati inégalement tendue, il oublie qu'une lame tormée dr cellules, bien qu'elle possède une certaline elasticité, constitue cependant un organe beaucomp plus complexe; il oublie aussi que les processus du plissement et de l'éragination sont, en tonte prenière ligne, déterminés par le pouroir d'accroissement de rroupes spéciaux de cellules: on ne peut donc les comparer aux inflexions et aux extensions d'une lame élastique.

Comme PAxder déja l'a fait observer, on ne doit pas s'imasiner yu'il s'argise du plissenent de menbranes inorganisées; mais les plis sont plutot eux-memes d'origrine oromique, c'est¿-dire engendrés, en certains points déterminés, par une multiplication cellulaire locale (1).

Celat étant établi, nous pouvons conclure que la localisation des alfëerencialirnas consiste dans ln! lixalisation, en des points

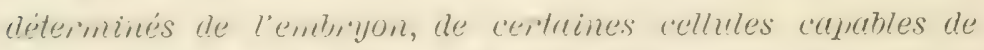

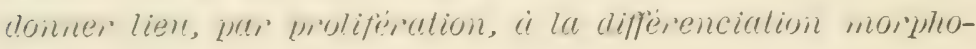
logique.

En résumé, nous établirons donc:

10 Les phénomènes fondamentan ade l'ontogénèse sont les quatre suivants: $1^{\circ}$ ) la prolifération cellulaire; $2^{\circ}$ ) la dif-

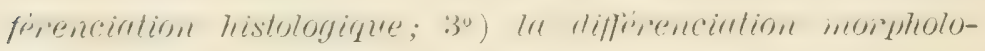
gique; $\left.4^{\circ}\right)$ la localisation des différenciations.

$2^{\circ}$ Ces quatre phénomènes fondanentaux sont conco-

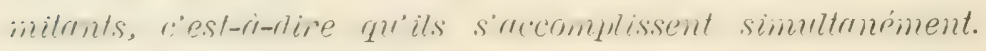
Ils sont en outre élroitement reliés entre eux.

$3^{\circ}$ La prolifération cellulaire consisle dans l'augmentation du nombie des cellules provenant d'une seule cellule: l'oeuf.

(1) Hertwig O. - Traité d'Embryologie etc. Paris, 1900, p. 206. 


\section{$-27-$}

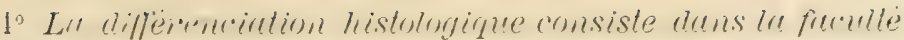

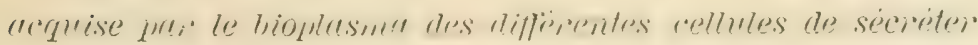
des substances spéciales.

$5^{\circ}$ La diffèrenciation mor hologique consiste surtoul dans

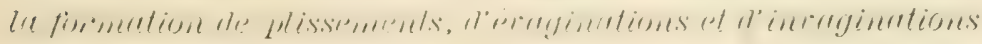
des feuillets germinatifs.

$6^{\circ}$ La localisation des differencentions consiste ans la localisation de la prolifèration cellulaire el des diffèrenciations histologique et morphologique. 


\section{Chapitre II.}

\section{La différenciation chimique.}

SOMMAIRE : La différenciation histologique est une sécrétion - La nature glandulaire de toutes les cellules - La sécrétion est le seul et lo vrai caractère de la différenciation histologique - Toutes les cellules vivantes ont leur différenciation histologique - Les propriétés des substances sécrétées ne sont pas des caractères d'importance dans la différenciation histologique - La diversité de constitution chimique des substances caractérisant la différenciation histologique - Importance de l'arrangement atomique dans les propriétés physiques, chimiques et physiologiques des substances - Différences dans la constitution chimique des substances possédant les mêmes propriétés physiologiques - La cause des différenciations histologique et morphologique est la différenciation chimique - La cause de la localisation des différenciations est la localisation de la différenciation chimique - Résumé.

Les connaissances acquises au chapitre précédent sur la réritable nature des différenciations histologique, morphologique et sur la localisation des différenciations, nous permettent maintenant d'en nieux approfondir l'étude et d'en reconnaître la cause intime.

Nous arons ru que la différenciation histologique n'est, en dernière analyse, qu'un phénonène de sécrétion, de la part Iu bioplasma, de certaines substances particulieres, qui penrent rarier extraordinairement par leurs propriétés chimiques et phrsiologiques, dans lesquelles résident précisément les caractères de la différenciation.

En partant de cette définition, nous' pourons conclure que chaque cellule de l'ortanisme, consilderé à une périorle quelconcune dre son existmene, pusiale uno différenciation qui lui est propre. En effet, comme nons arons ru, dams la le partie de ce tratail, due la sicrétion est $11 n$ phénomene accompa- 
gnant lassimilation (1), conme d'autre part nous savons que lassimilation est la faculté caralctéristique de la substance virante, il est bien érident que toute cellule virante, puisqu'elle assimile et sécrète, prosenterat une différenciation histologique determinie, et derra itre consilérée comme une glande. L'èlahoration au sein du protoplasma diune substance définie - a dit tout récemment encore RAxviER - est l'acte sécrétoire par excellence. A ce point de rue, toute cellule virante est une cellule rlandulaire, car toute cellule rivante élabore dans son intérieur nu certain prorluit quelle utilise ou rejette » (2). Et RANYIER a parfaitement raison.

Arec cela, je ne veux pas aller jusqu'à donner le nom de glande it toutes les cellules de l'organisme. Je m'en tiens seulement a la constatation que, toute diflërenciation histologique étant une sécrétion, il n'y a pas de cellules virantes sans une différenciation, quelle qu'elle soit.

La sécrétion est le seul caractère qui puisse nous permettre une définition exacte de la différenciation, les propriptes physiques, chimiques, morpholuginues et physiologiques des diverses substances sécrétées n'étant que des caractères d'importance tout à fait secondaire.

Lorsulue nous parlons de différenciation hiscologique, nous levons faire alsolument alsstraction les proprietés des substances qui lia caractérisent: l'autant plus que l'importance de cess propriétés dans la physiologie générale de lorganisme ne peut itre éraluée toujours exactement et, parfois, peut mème nous échapper complètement.

C'est ainsi, par exemple, que certaines membranes brutes, quoique dépourrues des propriétés contractiles on sensitives, ne sont pas moins importantes pour la vie de la cellule, grace aux phénnmènes d'osmose 'qu'elles peurent provoruer et dont

(1) Giglio-Tos E. - Les problèmes de la vie. 1e Partio, La substance vivante et la cytodiérèse. 'Turiu, 1900.

(2) Duval M. - Précis d'Hislologio. 1897, p. 285. 
nous arons éturlié le role an chapitre $V$ de la $I^{\text {e partie de }}$ ce travail.

De même, la graisse des cellules adipenses n'a pas de fonctions actives dans l'organisme; cependant elle a une grande importance, pourant, par son accumulation et par sal constitution chimique, constituer un réserroir de matériel qui, plus tard, donnera lieu a des substances particulières nutritives.

bans l'hémoglobine des érthrocrtes, nous arons l'exemple dune sulstance jouant un role plus actif, grice a sat constitution chimique. En formant des combinatsons spéciales arec loxruène ou arec l'anhyidride carbonique, elle sert à l'échange de ces deux gaz dans les tissus.

Enfin, dans la substance musculaire sécrétée par les myohastes, nous trourons un exmple l'me matiere très active, qui, par des transformations chimiques spéciales, accompagués de changenents morphologiques, se contracte et proroque des mourements.

La conception de la differenciation doit donc stre indépendinte des propriétés des substances sferétées, de mème qu'elle ne doit pas être subordonnée à la présence plus ou moins facilement constatable de celles-ci.

Ea effet, nous sarons que certaines substances liquides sécrétées par la bioplasma peurent diffuser à travers la membrane de la cellule. S'il en est ainsi, elles diffuseront à l'extérieur de celle-ci aussitist qu'ellos seront produites. Elles ne s'arcunulerout dnnc pas dans lia celiule. et peut ètre échatplepmenteles completement à nov recherches. In concluronsmon: que cette cellule ne possede pats de différenciation histologique? Nous commettrions une grave elreur: car cette

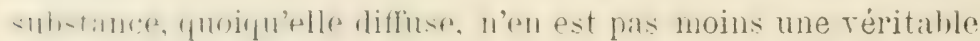
repretion lu hioplasma, fout comme les autres qui, it cause de leur nature chimique, s'accumulent dans la cellule ou bien sont de quelque manière apercerables par nos morens d'observation.

Lorsqu'on dit, par exemple, que l'oeuf est une cellule in- 
lifférente, cest-it-lire sans diflërenciation histologique, on lait usage dune expression trix inexacte. Ire mème que les: cellules adipeuses, comme nous l'arons ru, ont une différenciation histologinue parce pur leur hioplasina a lat facultéde sécréter de la graisse, de même aussi, nous derons conclure que l'oeuf a sa propre différenciation histologique caractérisée par les sulsstances dentmplasmilques qui sont, elles anssi, des produits de sécrétion du bioplasma orulaire.

Toute cellule, par le fait mème qu'elle a une constitution chimique définie, qu'elle est rivants et patr consingunt qu'elle sécrète, lossède une ditrérenciation histoluginue: ce qui n'entpèche pas qu'elle puisse la perdre et en acquérir une autre.

C'est dire qu'il n'existe fus me différenciation histoluginues dans le sens absolu, mais seulement dans le sens relatif, la nature de lit diffrenciation ditnt tominurs dipendante de la phase à laquelle on considère l'organisme.

Cela étant posé, on comprend parfaitement que la diffé-

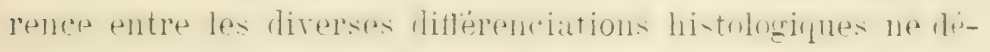
pend que de la diffẻrence entre les substances sécrétées par les cellules et caractéristiques de leur différenciation. Ces

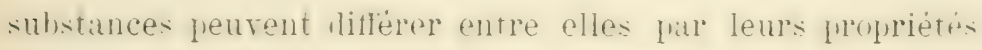

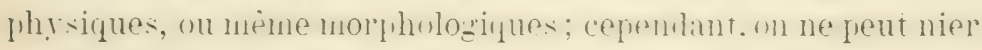
que ces propriétés sont, en dernière analysse, la conséquence d'une dirersitis dims leur constitutinn chininge. Il est a peine nécessitire de fatre rematruer, par excmple, que lat différence dans la coloration entre les sulatanes pigmentaires fropriptis physique) est due à leur nature chimique; que l'imperméa-

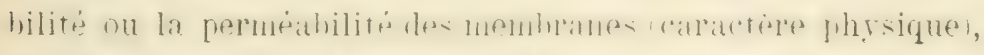
est étrutement liee a leur mostitution chimique: que la stru-

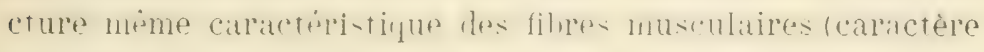

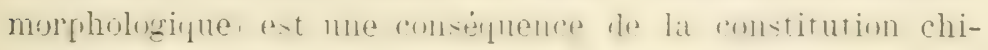
mique particulière de la substance contractile.

or, quelle est la cause de la dirersité dans la constitution chimique de ces substances? Yous pourons la connaitre facilement pour peu que nous reflechiscions it leur mole dorigine. 
Nous arons vu, au chapitre III de la le partie de ce travail, que la nature des produits de sécrétion peut dépendre en partie de lat composition chiminue des substances nutritives, mais yu'elle est surtont et plus particulièrement dépendante le la constitution du bioplasma qui les sécrète. Il est donc ivident que la nature des substances caractirisant la différenciation histologique dépendra essentiellement de la constitution du bioplasma des cellules, et, par suite, que les diflërences entre ces substances nous révèlent une différence correspondante entre les bioplasmas des cellules qui les sécrétent. Doù l'on conclura yu'i une différenciation histologivule détermine correspond une constitution bioplasmatique bien déterminée, elle aussi.

C'est lit une conclusion dont personne ne roudra contester lexactitude. ceprendant, je sens ici la nécessité de faire à ce frropos quelques remarques, afin d'éviter ultérieurement toute équiroque possible.

Si l'on peut aflirmer avec raison qu'une diversité dans les différenciations histologinues inporte nécessatrement une diversité dans la constitution chimique des bioplasmas, on ne peut toutefois en conchure réciproquenent qu'une difrèrence entre la constitution chinique les bioplasmas donne toujours origine it des diffirenciations histologinuement diflërentes. In moins, cette conclusion ne serait exacte que si nos connaissances sur les substances caractérisant ces différenciations histolorriques étaient appurées sur leur véritable composition chimique.

Mais comme nos moyens d'observation ne sont pas encore sumisants pour ce but; comme, au contraire, nous devons nous borner dans nos recherches à juger de la nature des ces substances, non pas d'après leur composition chimique, mais seulement d'après lomrs proprintés physiologiques, physiques on meme chimirques, il s'ensuit que leur connaissance est tonjours imparfaite et qu'il faut bien se garder de tomber dans des conclusions erronées. 
La ressemblance, et parfois l'égalité même des propriétés physiologiques, physiques et, dins une certaine mesure, des propriétés chimiques aussi, n'est pas un indice suflisant de l'identité de composition chimique des substances. Deux corps peurent fort bien nous apparaître égaux par ces propriétés, sans que leur constitution chimique soir pour cela parfictement identique.

Il faut se rappeler toujours que les propriétés physiques, et certaines propriétés chimiques ne sont pas étroitement dépendantes de la répitable composition chimique des substances, mais plutôt du type de leur composition.

Tout composé organique est caracterisé par trois facteurs: le nombre, la qualité et la disposition des atomes dans la molécule. Or, ce dernier est, de beaucoup, le facteur le plus important pour domer au composé ses propriétés phrsiques et chimiques.

Nous en arons des exemples frappants dans les composés métamères. Quoique dans cenx-ci le nombre et la qualité des. atomes de leur molécule soient les mèmes, leurs propripies sont néanmoins très différentes, et cette différence, nous le savons, n'est due qu'au différent arrangement atomique.

Au contraire, la ressemblance et parfois l'égalité des propriétés physiques et chimiques des composés sont dues a l'allrangement des atomes, a lat réritable structure moléculaire, indépendamment ou presque indépendamment du nombre et parfois de la qualité même des atomes qui les constituent.

Je crois qu'il n'est pas nécessaire d'en citer des exemples. Nons n'arons qu’i consulter les tratés de chinie organique, et nous verrons que la classification et la formation des groupes des composés orominues, présentant entre mux des analogies dans leurs propriétés, ne sont pas basées sur le nombre et la qualité des atomes, mais sur le mode de liaison réciproque de ceux-ci dans la molécule.

Cela étant posé, nous pourons armetre sans difficulté, que des substances possélant des fropriétes physiques, chimi- 
ques et physiologiques égales ou analogues n'aient pas parfaitement une composition chimique identique, mais seulement une analogie de structure moléculaire résultant du mode de liaison réciproque des atomes. Et si l'on considère encore que les molécules des substances caractérisant les différenciations histologiques doirent être d'une grande complexité et, par suite, constituées d'un grand nombre d'atomes, on conprendra facilement que ces substances, qui jouissent de proIriétés égales ou presque égales, pourront être très nombreuses.

I'ailleurs, l'interprétation même de l'assimilation que j'ai proposée dans la $I^{\text {e }}$ partie de ce travail ne saurait être acceptée si l'on n'admet implicitement une très grande variation dans la constitution chimique de la substance vivante pendant la période de l'assimilation, et cela, sans que ces changements chimiques nous apparaissent par quelques caractères qui puissent être décelés par nos moyens le technique microscopique.

Le groupe des substances albuminoides auxquelles appartiennent les molécules de la substance vivante peut comprendre plusieurs millions de composés différents; et pourtant, ceux-ci nous apparaitront ì peu près égaux, ru l'insuffisance de nos moyens dinvestigation chimique. Ia technique microscopique ne peut pas nous réréler les caractères chimiques des composés de la substance virante. Nous ne devrons pas conclure, par exemple, que la chromatine des cellules animales wet véritales a une constitution chimique identique, parce qu'elle se colore dius toutes ces cellules par les memes substances colorantes!

Du reste, bien que nos moyens de recherches dans cette sorte l'investigations chimiques soient encore insuffisants, et que des difficultes tres graves s'opposent it la connaissance exacte les substances sécrétées par les cellules, on possède séammons des preuves que cette différence de composition chiminure, dins des substances jouissant des propriétés analogues, est un fait réel. 
On sait, par exemple, aujourd'hui que l'hémoglobine des diver's Vertébrés, quoiqu'elle possèrle dans tous les mêmes propriétés chimiques bien connues, et par suite, la même fonction physiologique, a cependant une composition chimique différente.

Je suis profondément convaincu qu'une pareille conclusion s'étendra ì toutes les autres suhstances de sécrítion au fur et à mesure que les connaissances chimiques de la biologie progresseront. Je crois même que les Biologistes seront forcément amenés à cette conclusion que cette diversité de composition chinique des substances jonissant des mêmes propriétés physiologiques n'existe pas seulement entre les espèces différentes des êtres, mais encore entre les différents individus de la mème espèce, et, pour un mème indiridu, entre les différentes époques de son existence, et entre les différentes parties de son corps.

On pourrait dire que c'est là une exagération. Cependant nous n'arons qu'à examiner les phénomènes les plus communs et banals, pour nous convaincre que cette conclusion est, très probablement, une vérité.

Je crois bon de citer quelques exemples, qui n'auront d'ailleurs d'autre but que de faire mienx comprendre la signification exacte de cette conclusion.

La substance contractile caractéristique des fibres musculaires striées jouit de la mène propriété physiologique chez. tous les Vertébrés. cependant, personne ne pourra nier qu'il existe une différence chinique très grande entre les diverses espèces; diffẻrence qui échiplera peut ètre aux moyens de recherche scientifique, mais qui se rérèle à notre sens du gout par une différence de savent très marquée. "est d'ailleurs ce que nous pourous constater ausi entre les indiridus: différents d'une seule espèce.

La substance contractile ne nous manifeste pas de differences appréciables dans ses fonctions chez le mème indiridu à rles phases direrses de son existence; et pourtant, quelle 
diversite de sitrour, par exemple, entre les muscles d'un reau de lait et ceux d'un boenf! Cette différence de saveur n'est-

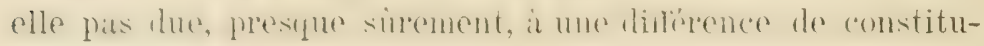
tion chimique?

Les recherches de la chimie biologique n'ont pas réussi

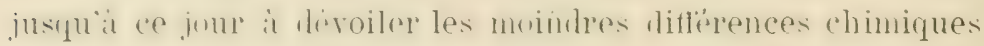
rntre les muscles des diverses parties du corps. rependant on ne pent nier que celles (ei existent. Xous en arons une preuve, far exemple. dans la dill̈érence de saveur entre les muscles lombatires filet de bendi) et les muscles de lat cuisse d'un mème hoeuf, un bien entre les muscles des cuisses et ceux des ailes d'un même poulet.

Je pourrais citel encore l'autres exemples analogues parmi les végétaux, mais je m'en tiens à ceux que je viens de mentionner. Qunique rulgitires, ils n'en sont pas moins instructils et ils suflisent à nous démontrer qu'il n’y a pas du tout d'exatgération dans ma conclusion ci-dessus énoncée.

Les considérations que je viens de faire ne devront jamais

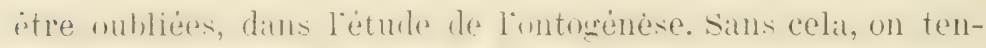
terait inutilement de trouver une explication de certains

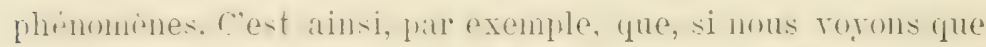
les collules loum blastule ont tontes la mene differenciation histologique, fuellos posingent toutes, par exmple, des cils ribratils, ou des flagellums, il ne faut pas en conclure que

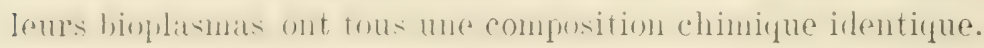
D’après ce que je riens de faire remarquer, une différence dims la constitution chimique hinmlasmatique de ces cellules est parlatement conciliable arec lougalité de leur diflérenciation histologique.

En conclusion, ce qu'il nous importe surtout d'établir c'est: flur les substances caracterisant les dillérenciations histologriques sont des produits de sécrétion du bioplasma des cel-

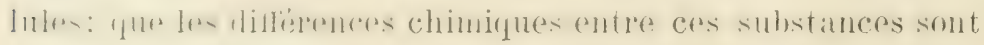
dues aux différences dans la constitution chimique des bioplasmas; qu'enfin, ces rlifférences chimiques ne se rérèlent 


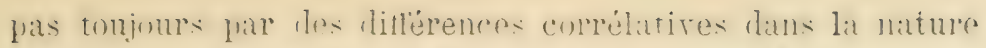

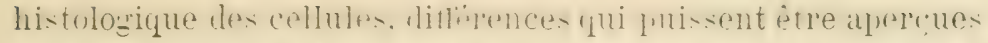

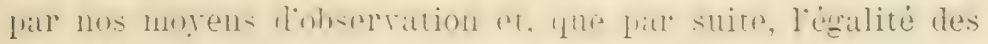

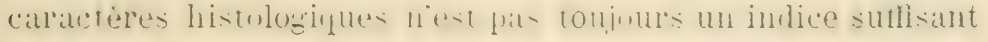
de l'égalité de composition chimique des bioplasmas.

On roit donc que lat diffirenciation hi-tuls ginne se réduit, en dernière analyse, a la diflërenciation chinique des bioplasmas des dirersps cellule- ré-ultant de lat sementutition de loeuf, et que. par conserquent. l'interpertation le ce tres ingurtant phe -

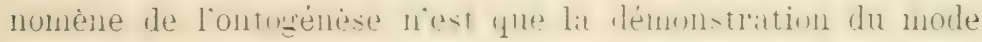
lar lequel cette ditlërenciation chininue punt setlectuer.

L'analyse de l'origine de la différenciation morphologique nous conduit à une conclusion analogue.

Nous arons tu au chapitre précédent que la différenciation morphologique consiste surtout dans la formation de plis-

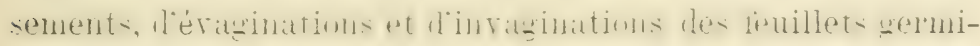
natifs. Or, ces feuillets sont bien des lames, mais des lames organiques, c'est-a-dire constituées de cellules, ainsi que PANder l'arait déjá remarqué. Bien que ces phénomènes de plissements soient donc un fait incontestable, on ne peut

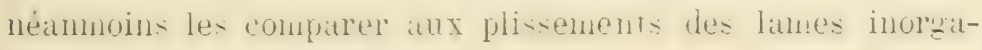
niques inégalement tendues.

Toute obserration embryolugiques, méne la plus simple, nuns

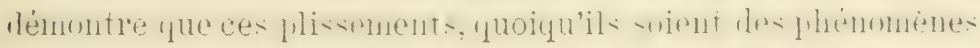

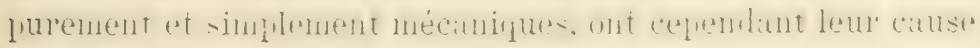

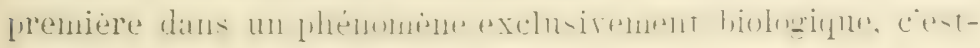
a-dire dans la prolifération cellulaire.

On sait, en effet, que les éraginations ou les inraginations

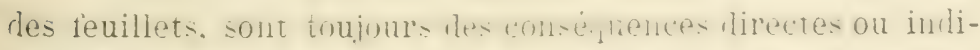

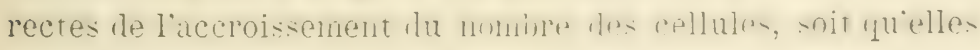
se manifestent dans l'endroit mème où l'accroissement a lieu

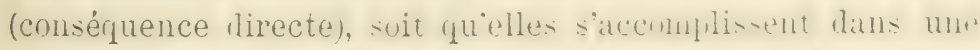

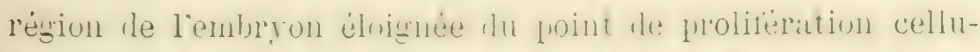
laire, mais toujours par l'action mécanique de celle-ci (conséquence indirecte). 
Chercher lit cause de la difrérenciation morphologique, c'est done la mème chose que chercher lir caluse de la prolifération cellulaire.

Or, celle-ci ne peut se faire sans assimilation préalable, condition indispensible pour le dédoublement biomoléculaire, pour le dédoublement biomorique et, par conséquent, pour la cytodiérèse.

si done nous royons que des plissements se produisent dans l'embryon, cela reut dire que la prolifération cellulaire est plus active; et si celle-ci est plus active, cela signifie que l'assimilation est, elle aussi, plus active, c'est-i-dire que les conditions phṛsico-chimiques nécessaires pour les phènomènes assimilatoires (conditions de vie) sont meilleures.

Mais nous avons $\mathrm{vu}$, au chapitre I de la $\mathrm{I}^{\mathrm{e}}$ partie de ce travail, que les conditions de vie sont de deux sortes: condition de rie intrinsèque et conditions de rie extrinsèques. La premiere est la résultante de la constitution chimique de la substance rirante; les secondes sont dommés par l'ensemble des conditions physico-chiniques du nilieu. Il est donc évident que, si la prolifération cellulaire est plus active, cela ne dépendra pas seulement des conditions extrinsèques, mais de la condition intrinsèque aussi, c'est-it-dire de la constitution chimique particulière du bionlasma des cellules oì l'on vient de constater une prolifération plus active.

La différenciation norphologrique étant une conséquence de la frolifération cellulaire dérive donc, elle aussi, de la constitution chimique du bioplasmal et son explication, tout comme celle de la différenciation histologique, se résout, en dernière analyse, at démontrer de quelle maniere certaines cellules de lembryon, issues par sermentation de la cellule-oenf primitive, ont acquis une constitution chimique de leu! bioplasma, capable d'une activité assimilatrice plus grande.

La diff̈renciation morphologigue se rérluit, en somme, elle allsi, at nus difremenation chimique du bioplasmat des cellules. 
Quant à la localisation des différenciations, nous pourons maintenant en trouver la cause efliciente d'après les considérations que nous venons de faire.

Pourquoi les différenciations histologiques et morphologiques se localisent-elles it les endroits déterminés et ne s'étendentelles pas à tout le corps de l'embryon?

Pourquoi, chez certains animaux, la localisation de ces phénomènes se manifeste-t-elle dans des aires à symétrie rayonnée et, chez certains autres, dans des aires à symétrie bilatérale?

$\mathrm{Si}$, comme je riens de le démontrer, la prolifération cellulaire, et les différenciations histologique et morphologique ne sont, en dernière analyse, qu'une conséquence directe de la différenciation chinique, il est érident que la localisation de ces différenciations n'est que la localisation de la différenciation chimique. si l'on voit donc que les phénomènes de prolifération cellulaire, et de différenciation histologique et morphologique se localisent a certains endroits de l'embryon, c'est parce que la différenciation chinique du bioplasma des cellules qui les produit est localisée à certaines cellules occupant, dans l'agrégat cellulaire qui constitue l'embryon, une position déterminée.

En résumé, nous pouvons donc conclure:

$1^{\circ}$ Chaque cellule rim ante possède toujours une diffërenciation histologique.

$2^{\circ}$ Il $n^{\prime} y$ a pas de différenciation histologique dans le sens absolu du mot, mais seulement dans un sens relatif $\dot{a}$ la phase à laquelle on considère l'organisme.

$3^{\circ}$ La canse des differenciations histologique et inoiphologique el de la protifération cellulaire, ieside dans la differenciation chimique du bioplasma.

$4^{\circ}$ La canse de la localisation des différenciations est la localisation de la différenciation chimique. 
$5^{\circ}$ Les quatre phénomènes föndamentanix de l'ontonénèse

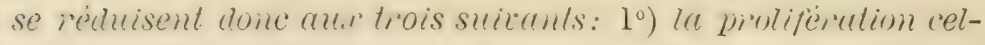

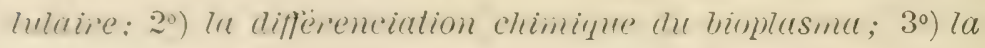
localisation de la différenciation chimique.

6. La differenciation chinique du bioplasina est donc le pitènoinène le plus important de l'ontogénèse. 


\section{Chapitre III.}

\section{L'otuf.}

SOMMAIRE: L'anité cellulaire de l'oeuf' fécondé - Son apparente absurdité -

La conception de la biomonade et son importance pour la compréhension do l'unité cellulaire de l'oeuf - La structure le l'oeuf et de ses parties - Le bioplasma - Le deutoplasma - Nécessité d'une exactitade la plus rigoureuse dans la distinction des partios ovulaires - Le vitellus formatif et le vitellus nutritif - L'action de la gravité et la disposition des parties de l'oeuf en. raison de leur densité - L'isotropie de l’oeuf - Résumé.

Arant d'atborder toute interprétation de l'ontogénèse, il nous reste encore à connaitre la constitution de loeuf, autant que le permettent nos connaissunces autuelles. Nous verrons mème que ces connaissunces, quoique inconplètes, sont suffisantes pour obtenir une explication satislatisante des principaux phénomènes ontogénétiques.

Fitisons abstraction, pour le noment, des phénomènes très complexes de la maturation des cellules génétiques, ce que je traiterai longuement dans une autre partie de ce travail, et bormons-nous à consirlérer l'veuf aprés la frénétration du spermatozoïde, c'est-á-dire après la fécondation.

C'est un fat incontestible que l'oeul, apres l'expulsion des globules polaires, est encore une cellule.

Il est aussi incontestable que le spermatozoude est une cellule autant que le spermatide, duquel il dérire.

En effet, ces deux éléments sexuels présentent toutes les parties qui caractérisent la callule. Hs sont ilonc des cellules.

Or, comme la fécondation est lit pénetration du spermato- 
zoide dans l'oeuf, les deux cellules sexuelles s'unissent dans cet acte, et l'veuf, après la fécomelation, ne sera plus constitué d'une seule, mais de deux cellules.

Fous arrirons donc, par ce raisonnement, rigoureusement logique, à la conclusion inévitable de la duplicité cellulaire de l'veuf. Et cependant, nous royons que, mème après la fécondation, loenf nous apparait toujours comme une cellule unique. L'apparition d'un seul fuseau de segmentation est une preuve suffisante de cette unité cellulaire de l'oetif fécondé.

C'est ici que nous pouvons, dès a présent, apprécier l'importance, je dirais mème la nécessité de la conception de la biomonade, telle que je l'ai exposée dans la $I^{e}$ partie de ce travail.

La cellule est une conception purement et exclusivement morphologique. Lal biomonade, au contraire, est moins une conception morphologique qu'une conception physiologique et dynamique.

La biomonade est un système symbiotique de biomores. Elle renferme donc implicitement l'ilée du nombre et de la nature bien déterminés de ces biomores et, en mème temps, l'idée des échanges mutuels qui doivent entretenir leur vie.

L'oeuf, après l'expulsion des globules polaires et arant la fëcondation, est une cellule et, en meme temps, une biomonade; le spermatozoulle est, lui aussi, une cellule et une biomonade. Mais si nous smpposons, ce que personne ne pourra contester, que les hiomores constituant cess deux biomonades sont différents entre eux, nous pouvons comprendre très ficilement que leur union pourra bien ne donner lien qu'it une seule biomonarle, c'est-i-dire a un seul système symbiotique de biomores, résultant de la fusion des deux systèmes difrérents qui constituaient l'oeuf et le spermatozoide.

Si donc au mot de cellule nous ne donnons qu'une signification purenent morphologique, ainsi qu'on le fait généralement aujourd'hui, nous sommes contraints d'arriver à la conclusion que l'veuf fécondé est une cellule double, ce qui est 
absclument contraire aux résultats de l'observation. Miais si nous attachons à la cellule la conception de la biomonade, nous comprenons parfaitement que l'oeuf fécondé peut bien n'être qu'une cellule unique, quoiqu'il résulte de l'union de deux cellules.

L'unité cellulaire de l'oeuf fécondé, est donc un fait réel et incontestable; mais nous ne la pourons comprendre exactement que par la conception de la biomonade. Grâce à celle-ci, il nous est possible de concilier entre eux deux phénomenes qui nous paraissent au premier aborl inconciliables: je reux dire l'unité cellulaire de l'oenf fécondé résultant de la somme de deux cellules.

Je m'en tiens, ici, à ces simples considérations et je me réserve d'expliquer ultérieurement, dans une antre partie de ce travail, comment se forment les deux cellules sexuelles et en quoi consiste la fécondation.

Pour le moment, nous derons faire abstraction de ces phénomènes et examiner seulement, si, dans l'oeuf fécondé, se trouvent les comlitions nécessaires pour le développement de l'organisme.

On sait que dans tout oeuf il faut distinguer toujours deux substances: le bioplasma et le deutoplasma.

Le bioplasma est la substance vivante. Il est constitué de biomores et ceux-ci de biomolécules, et comprend non seulement le noyau de l'oeuf, c'est-i-dire lit résicule germinative, mais anssi l'ensemble des biomores cytoplasmatiques qui sont groupés généralement autour du noyau. C'est donc dans le bioplasma que s'accomplissent les rrais phénomènes de la rie. Il est sans aucun doute lia partie la plus importante de l'oeuf. Le deutoplasma, au contraire, est une substance brute. Il est composé de matières spéciales sécrées par le bioplasma de l'oeuf avant l'achèvement de sa maturation. Ces matières ne sont le siegre d'aucm phenomene vital: cependant, elles foumissent, par leur présence, les conditions chiniques nécessaires à la vie du bioplasma. 
Il y a donc des relations très étroites entre la constitution du bioplasma de loesuf et la nature de son dentoplasma. Toutefors, il est indispensahle de fatre toujours une distinction bien tranchẻe entre ces deux sortes de substances ovlilaires.

Cne exactitude lat plus rigoureuse s"inpose alosolument dans l'emploi des termes relatifs a ces parties, et c'est cette exactitule qui manque parfois a la plupart des Biologistes, lorsuu'ils traitent de ces phénomènes. C'est ainsi, par exemple, qu'on appelle très souvent le «cytoplasma de l'oeuf", toute la partie de celui-ci en dehor's du noyau, et, par suite, la partie même qui contient le deutoplasma.

Or évidemment, cette expression est inexacte, et elle pourrait ètre une des causes empèchant l'interprétation des phénomènes ontogénétiques et notanment de certaines expériences d'ootomie.

En effet, le cytoplasma est la partie de la cellule en dehors du noyau; il est une partie de tout le bioplasma de la cellule: il est donc virant. C'est donc á tort qu'on appelle de ce nom le deutoplasma de l'oeuf, qui n'est pas vivant.

Le vrai cytoplasma de l'oeuf est l'ensemble des biomores constituant son bioplasma en dehor's des biomores karyoplasmatiques. Il n'est done qu'une partie tries petite de toute la masse ovulaire.

Il fiant se r'appeler toujour: yue l'oent' mûr, quoiqu'il possède parfois de grandes dimensions, n'est à l'origine qu'une cellule microscopintue, ef que son accroiscrment, ainsi quon peut le constater aisément par l'observation directe, n'est pas dû a l'augrmentation de la masse biophasmatique qui constituat la cellule-oeuf arant le commencement de son érolution, mais a la prorluction continue les substances deutoplasmatiques.

Il est donc évident que la masse bioplasmatique de l'oeuf, á l'achèvement de sa maturation, ne sera pas plus grande qu'au commencement. Elle est même diminuée, si nous considerons fur l'expulsion du denxième globule polaire réduit de la moitié la masse virante ovulaire. Il est vrai que la fécon- 
dittion, c'est-itrlire l'union du spormituzoide, compense cette diminution; mais, en tout cas, mème après cette compensation, la masise totale bioplatunatique de l'oeuf féconde ne sera pas plus grande que celle qu“il possedait lorsulil n'arait pas encore sécrété les substances deutoplasmatiques.

C'est donc une erreur que d'appeler cytoplasma orulaire ce qui n'est en réalité que du deutoplasma, et il faut absolument distinguer, au mons théniquement, ces deux parties, mime lorsque, pratiquement, cette distinction n'est pas possible.

J'avoue que, dans quelques oeufs, il y a parfois un mélange tris intime de parties bioplasmatiques et deutoplasmatiques: mais on ne peut nier" 'que, dans lat plupart des veufs, not amment dans ceux qui sont pourvus de deutoplasma abondant, la distinction entre le bioplasma et le deutoplasma est toujours possible et que, dans quelques cas, elle est mème facile.

Inas ces oenfs, apres l'anission du denxieme globule polaire, le bioplasma se rétracte, et tous ses biomores vont se grouper dans une petite partie de l'oeuf, formant la résicule germinative et son cytoplasma environnant. C'est ce qu'on roit d'ailleurs très distinctement dans les oeufs télolécithes.

Cet isolement du bioplasma orulaire persiste même jusqu'à la première sementation, oi les bionores binplasmatiques. s'orientant pour donner lieu à la cytodiérèse, contractent des rapports de position arec les particules deutoplatsmatiques et, par suite, s'entremêlent arec elles.

Peut être le lecteur n'arrire-t-il pas à comprendre, pour le moment, pourquoi jinsiste sur cette particularite de disposition des parties de l'oeuf. Il en rerra plus tard la nécessité.

II nous reste maintenant à examiner la constitution du deutoplasma.

On sait que celui-ci est formé de deux parties: le vitellus formatif et le ritellus nutritif.

Le vitellus formatif ne fait jamais défaut dans tous les oeufs, pourant être cependant plus ou moins abondant. 11 est généralement constitue d'un liquirle de nature albuminode, dans 
lequel peurent se trourer en suspension des granules très petits de substances spéciales jusqu'ici peu connues.

Soit it cause de sa constitution physique, soit encore a cause de l'absence presque complète de coloration, le vitellus formatif ne peut, parfois, etre distingué facilement du bioplasma. Il arrive même très souvent que le vitellus formatif et le hioplasma s'entrentent d'une maniere si intime, ou du moins yu'ils contractent des relations de position tellement étroites, que la distinction des limites de séparation des deux substances n'est absolument plus possible.

Le vitellus nutritif est formé, en prévalence, de granulations plus ou moins intmites. Il est quelquefois en très petite quantité, par exemple dans les oents alécithes; le plus sourent, il est tress abondant, ainsi que dans les oeufs télolécithes, et dans ce cas, il est généralement, à cause de sa densité plus grande, accumulé au pôle inférieur de l'oeuf.

C'est bien à raison qu'on appelle formatif la première sorte de vitellus. Il jone en effet un grand rôle dans la formation directe de l'embryon et dans la différenciation des cellules, ainsi que nous le verrons; tandis que le vitellus nutritif sert seulement de nutrition à l'organisme, lorspue la différenciation est accomplie ou presque accomplie. Celui-ci n'est en somme qu'une matière de réserve, qui joue un rôle tout-á-fait secondaire dans les phénomènes des différenciations ontogénétiques.

L'étude de la disposition que le bioplasma et le deutoplasma presentent danc l'oenf ast d'une impontance capitale pour nous, parce qu'elle touche de très près à la question de l'isotropie ou de l'anisotropie de l'oeuf, qui a été le sujet de tant de discussions biologiques modernes.

Or, cette disposition est variable suivant les oeufs des différents animatux, et elle est surtont dépendante de lat quantité et de la nature de la substance dentoplasmatique.

On sait, par exemple, que, dans les oeufs alécithes, le bioplasma et le deutoplasma sont presque mélangés entre eux, on du moins qu'il n'y a pas une accumulation bien marquée 
de l'un on de lautre dans un point ytrelconque de l'oeuf. On sait aussi, au contraire, yue, dans les neufs télolécithes, l'accumulation du deutoplasma au prile riatatif est bien visible et frappante. Mais ce qu'il importe surtout de remarquer, c'est que cette accumulation, ainsi que les expériences l'ont démontré très clairement, n'est due, dans lit plupart des cas, qu’à la différence entre les densités du bioplasma et du deutoplasma.

Cela est déja presque suflisant pour exclure une véritahle organisation dans loeuf, la disposition diffirente que ses parties présentent n’étant que la consëuluence de l'action le la gravité sur celles-ci et pourant, par suite, changer sans empècher le développement de l'organisme.

Dans la plupart des cas, la densité du bioplasma est infëpienre a celle du deutoplasma. s'il en est ainsi, le bioplasma occupera dans loeuf le prile supérieur et le dentoplasma s'accumulera au pòle inférieur.

Nais nous sarons que le deutoplasma est constitué du vitellus formatif et hu vitellus nutritif. Or, le vitellus formatif est généralement moins dense que le vitellus nutritif et, par suite, sa densite diffère de la densite du hioplasma toujour. moins que celle du vitellus nutritif. Il s'ensuit que, dans les oeufs, le vitellus formatif s'accumulera en prévalence au poile supérieur, c'est-à-dire au mème pôle où se trouve placé le bioplasma, et le vitellus nutritif, plus lourd, occupera au contraire le pôle inférieur.

Cela nous explique pourquoi, dans les oeufs, il y a toujours des rapports de position très étroits entre le bioplasma et le vitellus formatit; pourquoi ils sont toujours mintremilés bien intimement, ainsi que je l'ai deja fait remarruer, et pouryuni encore le renversement des oeufs ne peut produire des perturbations persistantes dans leur constitution.

- Il est en effet très évident que, si l'on renverse un oeuf, le bioplasma, le vitellus formatif et le vitellus nutritif, vu leur mobilité, repremlpont peu i pex leur poition réciproyue par rapport au degre de lemr densite et se disposeront dans 
le meime ordre quils araient dans l'oeuf normal. De cette manière, l'oenf' reprendra sa première structure, et les perturbations produites par le renversement seront effacées par laction seule de la gravité.

Par la, je ne reux pas aller jusqu'á exclure une constitution propre à l'oeuf inlépendamment de toute action de la gravité. Je ne nie pas, par exemple, que, à cause de l'arlhésion ou de la cohrsion ou hien de quelques autres actions moléculatres, les particules deutoplasmatiques puissent aroir un arrangement. spécial, et, par suite, que lovenf possède une structure dépendant directement de la mature des particules qui les forment; mais je tiens à remarquer que, daus la plupart des cas, la disposition de celles-ci n'est soumise qu'à l'action do la gravité, et fue nous pourons la comprendre parfaitement daprès ce seul principe, sans recourir à d'autres forces spéciales.

En tout cas, même en admettant que l'oeuf possède un arrangement de ses particules, déterminé par la gravité ou par quelques autres actions, quelles qu'elles soient, j'exclus absolument, dans mon interprétation des phénomènes ontogénétiques, que cette disposition puisse aroir une relation quelconque arec la disposition. des parties et des organes de l'individu qui en dérivera. En d'autres termes: je refuse à l'oeuf toute anisotropie ayant la moindre relation avec les difrérenciations ontogénétiques et je suis, au contraire, partisan absolu de son isotropie.

J'espère démontrer, dans cette partie de mon travail, que la structure de l'oeuf est beaucoup moins complexe que nous ne le pensons, et que les phénomènes ontogénétiques et les résultats des expériences peurent trouver une explication satisfaisante, mème en admettant l'isotropie de l'oeuf.

L'organisation de l'ètre issu de l'oeuf, quelque complexe qu'elle soit, n'a pas la moindre relation avec l'organisation morphologique de l'oeuf.

S'il existe dans celui-ci une remarquable complexité, assurẻment nous devons la chercher moins dans sa structure mor- 
phologique que dans lit constitution chimique de son dentoplasma et notamment de son bioplasma.

Il faut enfin remarquer que, de mème que le cytoplasma orulaire est pourvu de sa réserve deutoplasmatique, le noyau, lui aussi, peut posséler une réserve de substances nécessaires à sa nutrition, substances qui peuvent s'accumuler à son intérieur et en augmenter le rolume, ainsi que nous le royons dans la plupart des vésicules germinatives.

Mais il est bien entendu, que, mème dans ces substances qu'on pourrait appeler le «leutoplasma nucléaire » je n’admets aucune disposition spéciale ayant rapport a la disposition des parties de l'organisme futur. En peu de mots, l'isotropisme de l'oeuf ne s'étend pas seulement à la constitution qu'il possède en dehors de son noyau, mais à son noyau mème. Loent tout entier est isotropique.

En résumé. nous pouvons, conclure:

$1^{\circ}$ Bien que l'oeuf fécondé résulte de l'union de deux cellutes, (l'oeuf mù et le spematozoüde), il est néanmoins une cellule unique.

$2^{\circ}$ L'unilé celullaire de l'oeur fécondé, laquelle nous apparaît comme une absurdilé, est au contraire parfaitement compréhensible par la conception de la biomonade.

$3^{\circ} L^{\circ}$ oeur est constitue de deux substances: le bioplasma, substance vivante, et le deutoplasma, substance brute.

$4^{\circ}$ Le deutoplasna est presque toujours constitue de vitellus formatif et de vilellus nutritif:

5" La disposition du bioplasma, du vilellus formatif et du

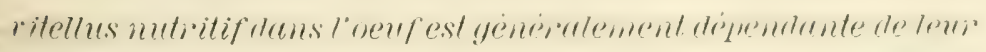
densité. Il s'ensuit que le vitellus de formation et le bioplasma contractent toujours des relations de position tres intimes.

$6^{\circ}$ Quelle que soit la structure de l'oeuf; elle n'a pas

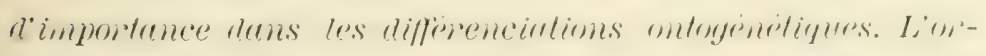
ganisation de l'être ne dépend pas de l'organisation morphologique de l'oeut. En un mot: l'oeuf. est isotrope. 


\section{Chapitre IV.}

\section{Les bases possibles de l'ontogénèse.}

SOMMAIRE: Existence dans l'oeuf des conditions chimiques nécessaires pour l'rssimilation - Nécessité des conditions physiques favorables - Importance et rôle du spermatozoïde dans la fécondation - Les bases possibles de l'ontogénèse: les développements biomoléculaires autogénétique, homogénétique, hétérogénétique - Examen de ces développements - Insuffisance des développements autogénétique et homogénétique pour l'explication des phẻnomènes ontogénétiques - Exclusion de ces modes de développement - Le développement hétérogénétique est la base de l'ontogénèse - Résumè.

L'examen de la constitution ovulaire que nous venons de faire dans le chapitre précérlent, nous lémontre qu'il existe dans l'oeuf deux substances: $1^{\circ}$ ) le bioplasma, substance virante, c'est-i-rlire capahle d'accomplir les phénomènes chimiques de l'assimilation; :2n) le deutoplasma et, plus particnlièrement, le vitellus formatif, substance brute, capable de réagir arec le bioplasma et de lui servir de nourriture. Il y a donc les conditions chimiques nécessaires pour toute réaction chimique.

Cependant, celles-ci, a elles seules, peuvent bien n'être pas suffisantes. Nous sarons en effet que toute réaction chimique exige aussi des conditions physiques spéciales, et notamment un certain degré de temperature, ou du moins une temprimature oscillant entre certaines limites, que nous appelons le maximum et le minimum. On sait aussi que ces limites sont rariables snivant la nature le lat réaction chimique qui loit s'accomplir. Il est donc évident que les réactions de l'assimilation entre lo bioplasma et le deutoplasma ne pourront commencer qu'alor's seulement que l'veuf serat placé dans des 
contitions physiques, et notiunment diuns des conditions de temperature firorahles, et tollos que la constitution chimique des deux substances l'exige.

Mais, comme celle-ci varie arec les oeufs des différentes espèces animales, nons jourons compremlre fatcilenent que le

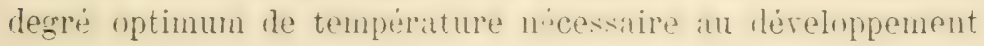
de l'oeuf doit, lui aussi, varier suirant les espèces que l'on considère.

On pourrait ici monjecter' : pournuni les réactions chintiques de l'assinilation et, par suite, du develnpenent ne commencent-elles pas dans la plupart des oenfs sans lëcomblation preatlable? Quel est le rile my ispienx que juue le spermatozoide?

La réponse à cette question exige malheureusement certaines connaissances sur la valour biolugique les bioplawnas de l'oeuf et du spermatozoide; connaissances que nous ne

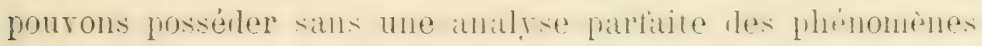
de la maturation sexuelle. Je dois donc rentoyer le lecteur à la troisième partie de ce trarail, ou je traiterai très particulièrement cet intéressant sujet.

Pour le moment, je me bornerai it lirène les fhénomènes de la maturation des cellules sexuelles aboutissent à ces resultats, que, apres les deux cytodirèses consieutires catractéristiques, loent' mur et le spermatozuile sont derenus deux biomonales incomplètes et complémentaires l'me le: l'autre. Incomplètes en tant que, à elles seules, elles sont incapables de se régénérer; complémentaires en tant que leur union forme une biononale complete, carable de se résénerer. au moins partiellement.

Or, nous sarons que toute biomonade est un système symbiotique de biomores, et je crois aroir démontré au chap. V de la $\mathrm{I}^{\mathrm{e}}$ partie, que la rie des biomores est rendue possible dans ce système, parce que les substances de sécrétion de certains dentre eux peurent servir de nourriture aux autres.

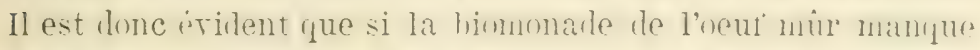

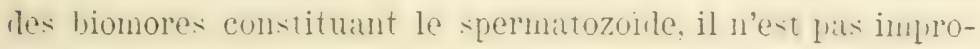


bahle yue les hiomores orulaires, i eux seuls, ne puissent pas acemplir leur dérelopnement et, par suite, que leurs biomolécules se puisent pas arrirer jusqu'au dédoublement.

Or, si celui-ci n’a pas lieu, les biomores et la biomonade non plus ne pouront se lédoubler et, par conséfuent, il n’y aturit lats te ertodieresse, cest-indire que la segmentation de l'oeuf' ne pourra pas s'accomplir.

Cependant je ne veux pas aflirmer que la fécondation soit toujour: une comlition indispensable pour le déreloppement des hiomolécules ovulaires. ('ela dépendra naturellement de la valeur des biomores du spermatozoïde par rapport aux hiomores le l'oent, c'est-it-dire de l'importance relative que lat frésence des biomores spermatozoïques peut aroir pour la nutrition des biomores ovulaires dans lo systeme symhiotique yu’ils constituent par leur union. Il n'est donc pas impossible que, dans certains cas, la nutrition des biomores de l'oent puisse s'acemplir, mème ì léfaut des biomores du spermatomirle, et alor's le dédoublement les biomolécules entranera le dérloublement des hiomores et celui-ci, la cytodiérèse.

La segmentation le l'veuf commencera, mème sans fécondation prealahle, ot donnera lieu a un de ces cas nom rares quon appelle, très sourent erronément, cas de parthénogénèse.

Je crois que ces considérations sont surlisantes pour domer une idée, quelque vagne qu'elle puisse être, du rôle que doit jouer le spermatozoile daus la fécondation, d'après non interpretation. Je regrette de ne pouroir ajouter ici al'atres explications, parce qu'rles exigeraient nécessairement la connaissance parfaite des phénomènes de la maturation sexuelle.

Revenons maintenant à l'oeuf fécondé, et supposons que les conditions physurue faromables an phemonemes chimiques de l'assimilation soient réalisées.

Ilors les hionolécules de l'ueuf, se nourrissant des substances Ju ritellus formatif, suivont leur diveloppenent, et aboutiont an déloublement en leux antres hionolécules. Cela entrainera 
le dédoublement des biomores et, atprès celui-ci, l'orientation biomorique produira la cytodiérése. Il s'ensuirra la segmentation de loneuf, suivant les lois rationnelles et suivant les solutions des problèmes que j’ai exposies dans la I" partie de ce travail.

Il s'agit maintenant de savoir si les biomolécules de l'oeuf se dédoublent en leux biomolécules igales entre elles et égales aux premières, ou bien en deux biomolécules égales entre elles et diférentes des premières; on bien encore en deux hiomolécules différentes entre elles ot différentes des prenières. En d'autres termes, il s'ayit de saroir si le léreloppenent suivi par les biomolicules est antogénétique, ou homogénétique, ou hétérogénétique (1).

Mais comme nos moyens actuels de recherche ne nous permettent pas de connaitre la constitution chimique des bioplasmas des cellules, nous sommes dans l'impossibilite de donner une réponse positive it cette importante question. Nous derons donc forcément recourir à des hypothèses.

T'outefois, celles-ci ne peurent être nombreuses, vu que tous les modes de développement possibles se réduisent aux trois types ci-dessus mentionnés. Il s'agit donc de roir lequel de ces trois modes de déreloppement biomoléculaire est suffisant pour nous permettre l'explication des phénoménes ontogénétiques. Par conséquent, nous les examinerons séparénent, en commençant par le développement autogénétique.

Nous pourons, pour plus de simplicité, représenter par des lettres la constitution chimique du bioplasma de l'veuf et des cellules qui dériveront de sil segmentation, e'est-il-dire lit nature chimique des bioplasmas résultant de l'ensemble des hiomolécules qui les constituent. Ainsi, par exemple, la constitution bioplasmatique de l'neuf peut itre représentée par la lettre $a$.

si le développenent biomoléculaire est antogénétique, les

(1) Veir I' Partie, Chap. II. 
constitutions bioplasmatiques des deux premières cellules résultant de lia segmentation, seront naturellement exales a celle de l'oeuf. La nature chimique des deux premiers blastomères derra done être indiqué frar ", ". En d'antres termes, lit fremière semmentation n'a fats été accompannée de Ja différenciation.

Cette première interprétation est généralement acceptée par la plupart des Biologistes, parce que quelques phénomènes et notamment les résultats de certaines expériences semblent plaider plutôt en sa fareur.

On sait, par exemple, que si l'on isole les blastomères issus des premières sementations de l'oeuf de plusieurs animaux (oursins, grenouilles, ete.), ceux-ci sont capables de former des cmbrrons complets, quoique plus petits. Ces résultats étant chtenus, roici le laisonnement suivi par les Biologistes: puisIue les blastonères isolés sont capables de produire le même effet final que l'oeuf entier, il faut bien admettre que la constitution des blastonères n'est pas difërente de celle de lonent. Ce ritisonnement, personne ne le peut nier, est en eflet très simple, ef tres logique. Il parait mème, an premier abord, qu'il doit exclure toute autre interpretation. Toutefois, je démontrerai tres clairenent dans les pages suirantes que les résultats ohtenus peurent itre expliques facilement, et d'une maniere plus complète, sans adnettre le développenent autogénétique.

l'ar contre, l'hypothèse de l'égalité de l'oeuf' et des premier's blastomères rencontre les dillicultés très grares, lorsqu'on reut explinuer les phénomènes ulterieurs de l'ontogenèse.

lin effet, si l'on admet que l'neut' est isotrope, et que le déreloplenent suiri par ses biomolécules est autogénétique, les deux jremiers blastomères seront égaux à l'oeuf non seulement pill leur constitution bioplasmatique, mais par la constitution reutoplasmatique aussi. s’il en est ainsi, les biomolixcules de ces hlistomieres lerront, olles aussi, suive le mîme développenent et, par conséquent, la seconde sergnentation 
donnera lieu ì quatre blastomeres égaux à l'oeuf, que nous pourons indiquer par la lettre ". Le mème résultat s'obtiendra après les autres segrmentations ultérieures, et l'agrégat cellulaire qui en dérirera, sera naturellenent composé de cellules possédant toutes la même constitution a de l'oeuf.

Comment expliquer alor's lia différenciation morphologrique et la localisation de cette différenciation?

Je suis bien disposé it supposer qu'après un certain nombre de segmentations, les substances deutoplasmatiques qui araient jusqu'ici produit le déreloppement biomoléculaire autogénétique soient épuisées, et que d'autres substances différentes puissent se substituer aux premières dans la nutrition du bioplasma et proroquer, par conséquent, un autre mode de déreloppement biomoléculaire. Mais, si ce phénomène a lieu, je ne rois pals pourquoi il derrait s'accomplir dans une seule des nombreuses cellules de l'agrégat. Puisque celles-ci sont toutes égales entre elles, ainsi que nous renons de le supposer, il n'y a pas absolument de raisons plausibles pour refuser aux autres les propriétés que nous accordons à l'une d'elles.

Dans ce cas, je ne saurais rraiment expliquer lal localisation des différenciations histologiques et morphologiques.

On a tenté aujourd'hui d'en donner une explication en faisant diverses hypothèses très peu scientifiques. On a supposé, par exemple, que les blastomères exercent entre eux des actions spéciales, d'où résulterait la différenciation. Mais je dois arouer que je ne puis arriver it comprendre, mème raguement, la nature de ces actions, ni leurs eflets non plus.

Vu le caractère purement expositif de mon trarail, je ne reux pas entrer dans des liscussions critiques sur les hypotheses des autres biologistes. Cependant, je ne puis m'empêcher le remarquer que, toutes les cellules itant égales, si une action quelconque est exercée par certaines d'entre elles sur les autres, la mêne action loit ître nécessairement exercée par ces dernières sur les premières. Et s'il eu est aiusi, nous 
sommes dans l'impossibilité d'expliquer la localisation des diflẻrenciations.

A ces raisons, qui forment un obstacle très sérieux à l'interprétation de l'ontogénese par le développenent autogénétique, on peut encore en ajouter une autre, moins positive et peut-ître plus philosophique, mais qui n'en est pas moins importante, je le crois, au point de rue scientifique.

Ainsi que nous pourrons mieux le comprendre plus tard, le but final de la formation des organismes, de l'ontogénèse, est colui, surtout, de créer un milieu interne tel que certaines cellules, que nous appelons les cellules germinatives, puissent arriver it régénérer ou l'oeuf ou bien le spermatozoïde dont elles sont dérivées. Si cela n'avait pas lieu, il n'y aurait pas de reproduction et, par suite, pas de vie.

Or, si l'oeuf était capable de se régénérer lirectement, ainsi yue nous le supposons dans l'hypothèse du développement autogénétique des ses biomolécules, à quoi bon la formation d'un oryanisme, quel qu'il soit? I)ans ce cas, l'oeuf suflirait de lui-même; il se trouverait dans les mêmes conditions qu'un nicrocoque, qu'une bactérie, en un mot, qu'un organisme inférieur unicellulaire capable de se régénérer directement en suivant le déreloppement autogénétique. La formation d'un ormanisme pluricellulaire, quelque peu complexe qu'il soit, serait un phénomène supertlu, lont je ne salurais trouver une explication saus recourir à des raisons plus ou moins téléologiques ou mystiques, et très peu scientifiques.

Je crois donc qu'alpés ces considérations, nous pourons exrlure de l'interprétation de l'ontogénèse l'hypothèse du déreloppement autogénétique des biomolécules de loeuf.

Cependant, si nous passons à l'hypothèse du dereloppement homogénitique, nous ne nous trouvons pas dans des conditions meilleures.

Dans ce cas, en elfet, puisque nous représentons la nature chintipue du bioplasma ovulaire far ", nous pouvons représenter celle du bioplasma des deux premiers blastomères par 
7), h, celle des quatre blastomères résultant de la deuxième segmentation par $c, c, c, c$, et ainsi de suite pour tous les autres blastonères. On voit facilement, que, dans cette supjosition, il y a bien de différenciation entre l'oenf et les blastomères; mais celle-ci est la mime dans toutes les cellules de l'agrégat cellulaire. Comment expliquer alor's la localisation ultérieure des différenciations? Nous trouvons ici les mêmes difficultés que dans l'hypothèse du développement autogénétique. Il fiut donc exclure de l'interprétation de l'ontorénèse l'hrpothèse du développement homogénétique (1).

Il ne nous reste maintenant à examiner que le développement hétérogénétique.

Nous sarons que le déreloppement biomoléculaire hétérogénétique aboutit à la scission d'une biomolécule en deux biomolécules inégales et différentes de la première. Par conséquent, nous pourrons indiquer la constitution chimique des bioplasmas des deux premiers blastomeres issus de la segmentation de l'oeuf, par les lettres $b, c$.

Or, je ferai avant tout remarquer que des trois modes de développenent biomoléculaire, ce dernier est, sans contredit, le plus simple et le plus fréquent parmi les phénomènes chimiques.

Je crois aroir démontré, au chapitre II de la Ie partie, que le dérloublement d'une molécule en deux autres égales entre elles est un phénomène chimique qui exige des conditions spéciales; conditions qui doivent résider en partie dans lat constitution mème de la molécule que l'on considère, en partie dans la composition chimique des substances qui doivent réagir avec elle.

Au contraire, le dédoublement d'une molécule en leux autres inégales est un phénomène chinique très commun, je

(1) Le développement homogénétique est suivi pent-être par quelques-uus do ces organismes unicellulaires infórienrs (pui vivent en colonies, dout les individus sont ou nous paraissent identiques. 
dirais même le plus commun parmi tous les divers changements chimiques de la matière. Il est donc fort probable que c'est sur celui-ci que les phénomènes ontogénétiques ont leur base; et cette probabilité acyuiert presque la valeur d'une certitule, si nous considérons que, par l'hypothèse du développement biomoléculaire hétérogénétique, les problèmes les plus ardus de l'ontogénèse peuvent trouver une solution satisfaisante très simple et très scientifique: ce que nous verrons dans les chapitres suivants.

En résumant, je conclurai:

$1^{\circ}$ Dans la constitution de l'oeuf fécondé existent les conditions chiniques néessaires pon les phènomènes de l'assimilation de la part de ses biomolécules.

$2^{\circ}$ Les biomolécules de l'oeuf ne peuvent suivre que l'un des trois morles de dérelopuement hiomoléculaire autogénétique, homogénétique, hélérogénétique.

$3^{\circ}$ Les drivelonpenents autogenélique et homngrinétique ne sont pas suffisents pou, nous erruliquer les phenomènes onlogénétiques.

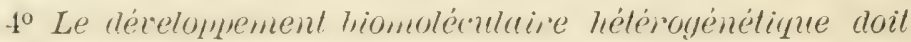
être nécessairement la base de l'ontogénèse. 


\section{Chapitre V.}

\section{Le développement hétérogénétique.}

SOMMAIRE : La potentialité évolutive de l'oeuf - Son origine et ses limites Les phases de l'évolution de l'oeuf - Le développement polyodique - Ses conséquences et son insuffisance ì l'explication des phénomènes ontogénétiques - Le dévoloppement monodique - Ses conséquences et son importance dans l'ontogénèse - Résumé.

C'est une notion chimique élémentaire et fondamentale que, les conditions physico-chimiques farorables étant données, la nature d'un composé quelconque résultant de la r'éaction entre deux corps est déterminée par la constitution de ces corps. On peut donc conclure que tout composé chimique préexiste potentiellement dans ses composants ou dans les corps dont la réaction donne lieu à sa formation.

Or, dans l'oeuf, les biomolécules du bioplasma et les substances constituant parr leur ensemble le deutoplasma ou, plus spécialement, la partie formative, le vitellus de formation, sont précisément des composés chimiques difrérents, capables d'entrer en réaction entre eux, lorsque certaines conditions physiques nécessaires à cette réaction seront réalisées. Alors, de lit part les biomolécules, commenceront les réactions caractéristiques de l'assimilation et les biomolécules subiront, par suite de celle-ci, res transformations chimiques qui aboutiront naturellement au dédoublement en deux biomolécules, que nous supposons différentes entre elles.

Or, la constitution chinique, quelle qu'elle soit, de ces deux biomolécules nouvelles sera évilemment dépendante de lit llature des réactions de l'assimilation, et celles-ci, à leur tour, de 
lit composition chimique du bioplasma et des substances du vitellus formatif qui leur ont servi de nourriture.

Fitisons, pour le moment, abstraction du phénomène du dédoublement, que nous examinerons sous peu, et considérons seulement la transformation subie par les biomolécules orulaires après la période d'assimilation.

Si nous appellons a l'ensemble des biomolécules constituant le bioplasma orulaire arant le commencement de l'assimilation, nous pourons indiquer parr th l'ensemble des hiomolécules du bioplasma après les réactions chimiques de l'assimilation. Ces leux lettres $\alpha$ et 7 représenteront la constitution chimique du bioplasma ovulaire avant et après la le période d'assimilation.

Les biomolécules du bioplasma $h$ se trouvant toujours en contact arec les substances deutoplasmatiques de l'oeuf, qu'on peut indiquer complexivement par $x$ et que je suppose toujour's les mèmes, parce qu'il n'y a pas de raisons de les supposer différentes, pourront entrer en réaction avec celles-ci et commencer ainsi une autre période d'assimilation.

Mais, si les biomolécules $a$, en réagissant avec les substances deutoplasmatiques $r$ de l'oeuf, ont domé pour résultat final, après la première période d'assimilation, des biomolécules d'une constitution 7, ces biomolécules 7, en réagissant arec les mêmes substances deutoplasmatiques $x$, arriveront à une constitution chinique quelconque, mais qui sera nécessairement diférente de h. J'appellerai donc e la constitution chimique du bioplasma après la deuxième période d'assinilation.

De même, les biomolécules c, en réagissant avec les substances deutoplasmatiques. $r$, aboutiront, après la troisième périonle d'assimilation, à une constitution quelconque, différente de ", et que j'appellerai d, et ainsi de suite. C'est dire qu’i chaque périorle d'assinilation, la constitution chimique du bioplasma sera lillérente de celle qu'elle possédait auparavant, et cette variation pourra continuer plus ou moins.

Ainsi donc, pour concréter nos idées, nous pouvons repré- 
senter la série de transformations suhies par le hioplasma ovulaire, par la formule suivante: $a \ldots . . . \quad b \quad c \ldots d \ldots e \ldots f \ldots g \ldots$ en se rappelant toujours yue ces lettrer representent la nature chimique de l'ensemble des hinnolécules a la fin de chaque périorle assimilatrice, et que chacune le ces étales est séparée de celle qui suit par une cytodiérèse que nous arons, pour le moment seulenient, négligée à dessein.

La série de ces transformations pourra néannoins s'arreter four une de ces trois causes: on bien, par l'epuisement des suhstances reutoplasmatiques nourrissant les biomolecules; on hien, parce que le bioplasma est arrivé, par les changenents successifs, à une constitution chimique telle que toute réaction arec le deutoplasma est ultérieurement impossible; ou bien encore, parce que les conditions phrsiques extérieures ne sont plus sullisantes a la réaction entre le dentoplasma et le hioplasma.

On roit, d'après ces considérations, que la nature chimique du bioplasma à la fin de la prenière période assimilatrice est dependante de la constitution " du hioplasma ovulaire arant cette periode et de la constitution r du dentoplasma. Or, comme cette derniere ne change pats, la constitution r du hioplasma après la deuxième périorle assimilatrice, sera dépendante de la constitution " et, par suite, de la constitution "b du hioplasina ovulaire. Te cette manière, "lépendra de c. " le $1, f$ le ete. En conclusion, charque numelle constitution acquise far le bioplasma sera la conséquence directe de la constitution que celui-ci possédait dans la phase précédente, ef la constitution dernière a laquelle il peut arriver sera la conséquence de celles qu'il arait amparatrant ot, par suite, de celle que possédait le bioplasma orulaire initial.

Nous pourons donc conclure que, dans l'oeuf, existe une po-

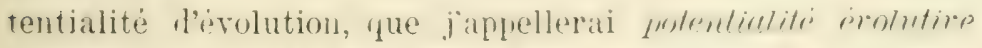
malnimer résultant de la constitution chinique du bioplasma et du deutoplasma, potentialité qui, étant réalisées les conditions physiques nécessaires, s'explique en amenant le bio- 
plasma orulaire, par une série de transformations suecessives, á posséder une constitution chimique tress difrérente de sal constitution primitive. Par conséquent, nous appellerons froIntion de loneuf la série de transformations que son bioplasma pent suivre a l'aide des substances deutoplasmiques contenues dans l'oeuf, et whases de cette érolution seront les constitutions chimiques différentes (7, c, 17, etc.) acquises successivement.

Cela étant posé, considérons maintenant ces transformations du bioplasma, non seulement en elles-mêmes, mais en relation arec la cytodiérèse qui, nous le sarons, s'accomplit à la fin de chaque période assimilatrice.

Puisque je suppose, ainsi que je l'ai dit au chapitre précédent, que la cytodiérèse est hétérogénétique, les deux cellules qui résulteront, après lit première période assinilatrice, du bioplasma ovulaire $\alpha$, seront différentes entre elles, et, comme nous arons indiqué la constitution chimique du bioplasma de l'une d'elles aree la lettre h, l'autre possèdera naturellenent un bioplasma de constitution différente de h. Mais quelle sera cette constitution?

lci encore, nous ne pouvons la connaître positivement; cependant, il est hor's de doute qu'elle sera nécessairement ou égale $i$ "ou différente de ‘, c'est-a-dire égale on différente de la constitution que nous arons attribuée au bioplasma après la deuxième périorle assimilatrice. Il n'y a pas d'autres sup)positions possibles.

Exaninons-les successivement toutes les deux et supposons, pour le moment, qu'elle soit diflérente de " et appellons-lat c'. Après la premiere cytodiérse, e'est-i-dire apres la première division de l'oeuf, les bioplasmas des deux cellules ou blastomeres résultants pourront donc itre indiquess par lo et r. Mais, comme je suppose que l'oenf est isotropique, la constitution du deutoplasma contenu dans ces deux blastomeres sera la même, c'est-d̀-dire $x$.

Or, nous arons vu que le bioplasma l, en réagissant avec le 
deutoplasma $r$, est capable de se transformer en $c$. Il est donc tout naturel que le hioplasma $c^{\prime}$, et par conséquent différent de 7 , en réagissant arec le mème deutoplasma $x$, ne puisse subir qu'une transformation différente de $c$.

Après la deuxième période assimilatrice, aura lieu une autre cytodiérèse, et, par suite, la division des deux premier's blastomères. Le blastomère 7 se divisera donc en un blastomère $c$, ainsi que nous l'avons démontré, et en un autre blastomère, lequel sera inévitablement égal à $d$ ou lifférent de celui-ci.

Supposons, ici encore, ainsi que nous l'atrons fait à la première division, que ce blastomère soit diflérent de $d$ et indiquons-le avec la lettre $d^{\prime}$. Le blastomère $b$ se sera donc divisé en $c$ et $d^{\prime}$.

A son tour, le blastomère $c^{\prime}$, en réagissant avec le deutoplasma $i$, arrivera i se diviser en deux atutres blastomères, qui seront naturellement différents entre eux, parce que nous supposons toujours que le développement est hétérogénétique, et différents des deux blastomères $c$ et $d^{\prime}$ dérivés de $b$. Le blastomere $c^{\prime}$ se divisera donc, par exemple, en deux hlastomères $d^{\prime \prime}$, et $e^{\prime}$.

Par conséquent, après la deuxième division, l'agrésat cellulaire dérivant de la segmentation de l'oeuf sera formé de quatre blastomères ayant la constitution bioplasmatique suivante: $c, d^{\prime}, d^{\prime \prime}, e^{\prime}$, e'est-à-dire qu'ils seront tous les quatre différents.

A leur tour, ces quatres blastomères, après une périorle assimilatrice, se diviseront et, suivant un raisonnement analogue à celui que nous venons de faire pour les deux premiers blastomères, on peut démontrer que $c$ se divisera en un blastomere $d$ et en un autre blastomère différent de e, par exemple $e^{\prime \prime}$; $l^{\prime}$ en $e^{\prime \prime \prime}, f^{\prime} ; d^{\prime \prime}$ en $e^{\prime \prime \prime \prime}, f^{\prime \prime} ; e^{\prime}$ en $f^{\prime \prime \prime}$, $g^{\prime}$, et l'agrégat cellulaire résultant après la troisième segmentation sera constitué de 8 blastomères tous différents entre eux.

On roit facilement que, par ce mode de déreloppement hétẻrogénétique, l'après lequel l'un des doux blastomeres issus 
de la crtodiérèse a une constitution chimique différente de celle que possèdera un des blastomères qui résulteront de la crtollérèse suivante, l’agrégat cellulaire dérivé de la segmentation de l'oeuf sera toujours formé, à une phase quelconque de son érolution, de blastomères différents entre eux. si fonc nous imaginons, par exemple, que l'évolution suivie par les hlastomères soit representée par des voies, sur lesquelles les blastomères s'acheminent rers la différenciation histologique définitive, ces roies seront aussi nombreuses que les collules de l'agrégat, et l'apparition le deux blastomères nouveaux après chaque cytodiérèse marquera aussi lapparition de deux nouvelles roies dans leur érolution.

Ce morle de développement hétérogénétique, je l'appellerai déreloppement polyodique.

Je ne sais si, dans l'énorme varièté des phénomènes biologiques naturels, il y a des organismes qui nous présentent ce mole de développement. S'il en était ainsi, toute différenciation histologique dans le corps de ces êtres ne derrait naturellement ètre représentée que par un seul élément, et, par suite, il y aurait autant de différenciations histologiques que de cellules. Mais dans l'état actuel de nos connaisiances biologiques, de semblables mpanismes nous sont inconnus. Le déreloppement polyodique n'a donc pour nous aucun intérèt; et cependant, je devais le mentionner afin le considerer tous les modes possibles de déreloppements que la nature peut nous présenter.

Revenons maintenant ì la prenière cytoriérèse de l'oeuf.

J'ai dit que, après celle-ci, il en résulte deux blastomères inéraux, dont l'un est bet dont l'autre est nécessairement, inéritahlement égal à eou différent de ce c'est-a-dire, égal à la constitution chimirue que le hioplasma orulaire a la potentialité d'acquérir à la deuxième phase de son évolution, ou hien différent de cette constitution.

or, nous venons de voir que si cette constitution de l'un des hastomeres ost differente de ", le développement est po- 
lyodique, et nous porte à des conséquences qui ne sont pas absolument alsurbes, mais qui ne peuvent pas expliquer les phénomènes ontonénétiques des olymismes que nous connaissons. Il ne nous reste donc qu'á faire l'autre des suppositions possihles, c'est-it-dire a suphoser que, les deux bastonères issus de la première division, l'un soit $b$ et l'autre égal à $c$ dans sa constitution bionlasmatique, ef par considuent, nous pourrons l'indiquer arec cette même lettre. Le bioplasma orulaire $a$ aura donc disparu pour faire place aux deux blastomères $b, c$.

A la deuxième segmentation, $b$ se divisela ell un blastomère $c$ et en un autre égal à d ou différent. Nais, comme nous venons de roir qu'en le supposant différent de $d$, on obtient le développement polyodique, il ne nous reste qu'i le supposer égal ì $d$.

De même, le blastomère $c$ se divisera à son tour en deux autres, dont l'un $d$ et l'autre égal à $e$ ou différent; et si, pour les raisons susdites, nous le supposons égal à $e$, les deux blastomères noureaux issus de $c$ seront $d, e$.

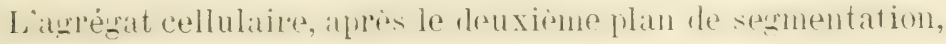
sera donc constitué des quatre blastomères: $c, a, d, e$.

Ainsi, si nous suirons le mème raisonnement, à la troisième cytodiérèse, chacun de ces blastomères se divisera en deux autres; c'est-i-dire: c en $l, e$; $l$ en $e, f ; d$ en $e, f ; e$ en $f, \theta$, et l'agrégat cellulaire r'ésultera formé de ces huit blastomères: $d, e, e, f, e, f ; f, g$.

Dans ce cas donc, en supposant que les deux blastomères issus de chaque cýtodiérèse présentent une constitution bioplasmatique égale à celle qui caractérise les phases immédiatement successives de l'évolution de l'oeuf, ainsi que nous l'avons démontré au commencement de ce chapitre, tous les blastomères ne parcourent pas, dans leur érolution, des roies différentes comme dans le déreloppement polyodique, mais une seule roie, et, plus précisément, la roie mème qui caractérise l'érolution de l'oeuf. 
J'appellerai donc ce mode de développement le déreloppement monodique.

On peut voir lideilement que, diuss le direloppement monodique, tous les bastomeres représentent, par leur constitution, une des phases de l'érolution de l'oeuf. On roit encore que, parmi ces blastomeres, quelque mombreux qu'ils solent, il y en a toujours un seul qui est plus arince que tous les autres dans l'érolution et que j'appellerai le blastomère en thite, et un autre qui est au contraire le moins avancé, et que j'appellerai le blastonère en quene, tandis que les phases de l'brolution, intermédiaires entre ces deux phases extrèmes, sont toujours représentées par un grand nomble de blastomeres, nombre qui va naturellement croissant au fur et it mesure que la segmentation progresse, et, par conséquent, à mesure que le nombre total des blastomères augmente.

D'autre part, on peut voir encore qu'à chaque division du biastomire en tîte, l'neuf progresse, non pars d'une semle, mais de deux phases dans son évolution, et qu'a chaque division du blastomere en quene, l'veuf abandonne une des phases primitives pour atteindre deux des phases successives de son érolution. Ire cette maniere, la distance entre le blastomère en queue et le blastomère en tête ra toujours croissant, en mène temps que la frogression de l'neul dans son érolution s'accélère à cause du développement monodique.

Je ne sais si le lecteur, par les seules considérations qui frreedrat, a deja connuris toute l'importance que le déveloprement monomique presente pour l'explication hes phenomènes ontogénétiques. l'eut-ètre n'arrire-t-il pas encore à comfrentre sil je venx aboutir arec cette nouvelle methorle denrisater l'ontugéneses. Cependint, je crois que, dans le cours de ce travail, il pourra se convaincre facilement que le déveluppenent momulique, tout simple qu'il est, noms permettra Hexpliquer aisement, arec frécision et sans l'airle l'hyputhress sréciales, les phénmenes ontogénétiques et l'en résoudre les problemes les plus compliqués. 


\section{En résumé :}

$1^{\circ}$ Louenf mosserte une potentinlitri érolutire, qui est deter-

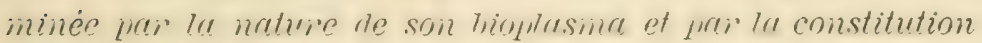

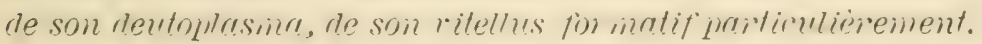

$2^{\circ}$ Si les conditions sont farorables, l'évolution de l'oeuf

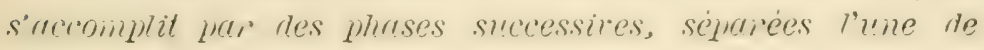
l'autre par la cytodiérèse.

$3^{\circ}$ Les blastomères issus de la segmentation de l'oeuf ne peurent suivie que deux modes de déreloppement: ou lé dé-

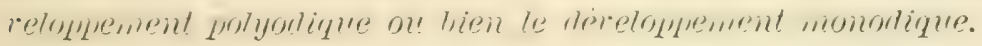

$4^{\circ}$ Le développement polyodique n'est pas suffisant à ex-

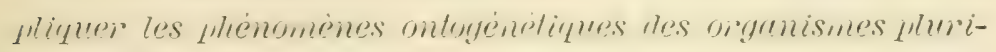
cellulaires commus. Il peut donc être exchu de nos considéiations.

5o Dans le déreloppement monodique, tous les blastomères suivent la mêne voie dans leur érolution, c'est-d-dire la voie qui caractérise l'érolution de l'oeuf.

$6^{\circ}$ Le déreloppement monodique est donc le seul qui puisse

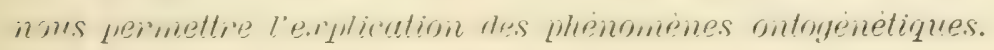




\section{Chapitre VI.}

\section{Le développement monodique.}

SOMnATRE: L'évolution de l'oeuf dans le dévoloppomont monodique - Lr ra pidité de cotte évolution - La phase limite et les phases intermédiaires L'hétérogéneitó de l'agrégat cellulaire résultant de la segmentation - Son retour possible à l'homogénéité - La déviation des collules de leur évolution primitivo - Résumé.

D'après ce que nous renons d'exposer dans les chapitres précédents supposons maintenant, pour concréter nos idées, qu'un oeuf quelconque soit capable de suivre une évolution, qui, par des phases successives $a, b, c, d, e, f \ldots .$. etc., l'amène jusqu'a la phase $l$. Si cet oeuf, ainsi que nous le supposons,

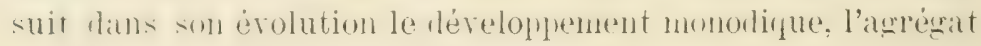
cellulaire résultant de sa segmentation sera constitué:

après la 1'segm. par les blastom, b
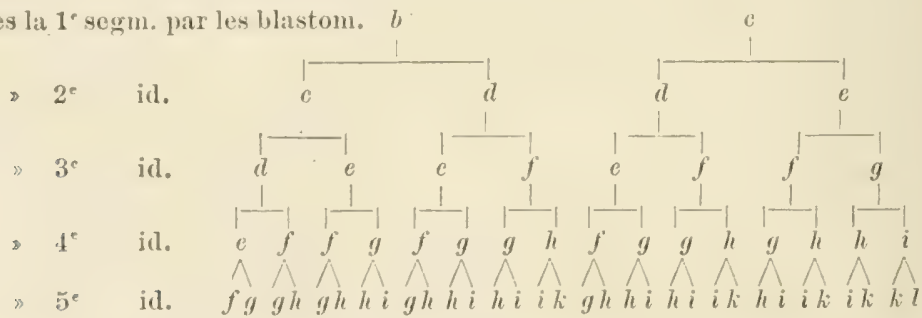

Après la $5^{8}$ segmentation, un blastomère de l'agrégat aura donc atteint la phase $l$, dernière limite de la potentialité érolutive de l'oeuf et, en supposant, pour le moment, que les cy-

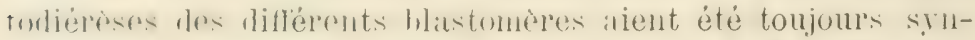
chroninges. l'asregat rellulaire résultera alor's comstitué de 32 hastoneres, dont un seul est encore i la phase fiet dont les antres se trourent dans des phases intermediaires, cest-it-dire 
5 dans la phase $/, 10$ dans la phase $h, 10$ dans la phase $i, 5$ dans la phase $k$.

Entre la phase $a$, point du départ de l'érolution de l'oeuf, et la phase $l$, dernière limite de sa potentialité évolutive, s'in-

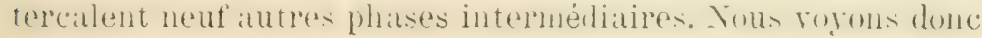
que toutes ces phases s'accomplissent par cinq périodes assimilatrices seulement et que le bioplasma orulaire franchit l'espace qui le sépare de la phase limite $l$, par cinq seules cytoliéreses successives, cest-it-rire arec une grande rapilite, limuelle noest que lat conséguence directe du déreloppenent monodique.

Or, comme nous arons démontré que toute cellule, à une phase quelconque de l'érolution d'un organisne, possèle une diflérenciation histolonique caractérisée par la nature chimique de son bioplasma, on peut dire que le blastomère $l$ possète une différenciation histologique l, et cette différenciation n'a été atteinte par ce blastomère qu'à la $5^{\circ}$ cytodié-

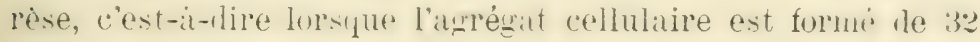
cellules. En outre, pour arriver ì cette différenciation, il a din passer prar les phases interminares. Nous pourons donc roir, dè il frésent, que toute niflërenciation histologique est etroitement lieje an nombres des cellules constituant l'argregat cellulatire all moment vil la diflérenciation fait son apparition, et qu'plle n'est que lit conséquence finale du nombre des phases intermédiaires de l'érolution de l'oeuf.

Puisque nous arons supposé $l$ la phase limite de la potentialité irolutive de l'oeuf, nous aroms arhnis inplicitement que le bioplasma l, par sa constitution spéciale, ne peut plus

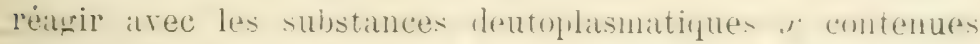
dans le hastomere I, mine en supposint fule cas sulstances,

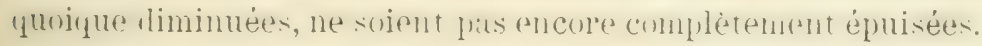
Le blastomère $l$ se trouvera donc arrêté définitivement dans son évolution. Il ne changera plus sa constitution. Il restera toujours l et ne pourra flus se diviser en d'antres blastomères. Mais il n'en sera pas de mème pour les autres blastomères. 
Le bioplasma du blastomère qui se trouve à la phase $f$ pourra bien encore réagir arec les substances deutoplasmattiques $x$ si celles-ci ne sont pas ípuisées, ainsi que nous pourons l'admettre. Or, comme les autres blastomères qui se trouvaient anpararant dans cette phase, après l'atsimilation, se sont divises en deux autres blastomères y, h, celui-ci, en continuant son assimilation, se dirisera, lui aussi, en leux blastomeres $y, h$.

De même, les 5 blastomères qui sont à la phase $g$ se diviseront il leur tour, chacun en deux blastomeres $h, i$. Ils donneront donc origine, par leur division, a j blastomeres het it 5 blastomères $i$.

Les 10 blastomeres $h$ en se divisant, chacun en deux autres blastomères $i, k$, produiront, par conséquent, 10 blastomères $i$ et 10 blastoneres $k$, et les 10 blastomères $i$ domeront, par leur division en k,l, 10 blastomères a la phase $k$ et 10 blastomères a la phase $l$.

L'angrégat cellulaire résultera donc constitué, après la $6^{\circ}$ division, d'un seul blastomère $g$, de 6 blastomères $h$, de 15 blastomeres $i$, de :0 blastomères h, de 10 blastomères l issus de cette $6^{\ominus}$ division, et d'un autre blastomère $l$ dérivé de la division précédente, c'est-a-dire de 11 blastomères l. A toutes ces cellules il faut encore ajouter les a blasiomeres $k$ issus de la division précédente et que nons derons considérer maintenant.

Ces j blastomères $k$ se diviseront-ils ultérieurement ou non? si nous armettons que l'assimilation de lat part le leur bioplasma soit encore possible, ils se diviseront encore pour domer origine, chacun, ¿̀ un blastomère l et it un antre blatstomère, qui sera nécessairement égal ì lou différent. Inans le premier cis, la cytodiérèse des blastonères $h$ sera homogénetique, ce que je ne crois pas probable, ru la dilliculté présentée par ce mole de développenent bionoléculatre. Inans le denxième cas an contraire, si l'on suppose le blastomère différent de $l$, et qu'on l'indique, par exemple, avec la lettre m, nous admettons implicitement dans l'oeuf une potentialité supérieure à celle 
que nous lui arons attribuée au commencement de ce chapitre: ce qui est contraire i notre hypothèse.

Qu'arriverat-t-il très frobiblement? (que ces blastonères $k$, ayant, d'une part, lat potent ialité de suirre leur érolution jusqu'a

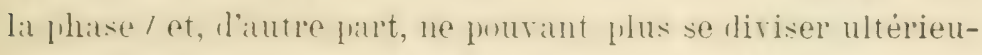
rement, se transtimemont en / sans se diviser et que, par suite, les 5 k produiront seulement 5 blastomères $l$.

bonc, aprés la 6 division, l'agrérat cellulaire dérivé de l'veut pusidelat is cellules représentant les phases de l'érolution de l'oeuf comprises entre $g$ et $l$. Les phases $b, c, d, e, f$ auront dispart complètement: mais la phatse linite $l$ ne sera plus représentée pall une seule cellule, mais par lis cellules, c'estin-rire que lat ditrerenciation histologripue, quelle qu'elle soit, caractérisét far lat enstitution du bioplatsmal, sera atteinte par 15 autres blastomères.

De même, à la $7^{e}$ division, le blastomère $g$ se divisera en $h, i$; les 6 blastomères $h$, se diviseront chacun en $i, k$; les 15 $i$ en $k, l$ et les $20 k$ se transformeront en $l$ sans se diviser. Le nombre total des cellules sera alors de 80 , dont 51 a la phase limite $l$.

Après la $S^{e}$ division, $h$ aura donné $i, k$; les 7 blastomères $i$ auront donné $\tau k$ et $7 l$, et comme les 21 blastomères $k$ de la dirision précédente se seront transformés en $l$ sans se diriser, il y ana dans l'anrégat des segmentations . $1+21+i=79$ cellules it la phatse limite/, et le nombre totil des cellules sera de 88 .

Enfin, à la ge dirision, $i$ se dirisera en $k, l$, les 8 blastomeres $k$ se seront transformés en $l$ sans se diviser, et l'agrégat résultera constitué le $7 !++8+1=88$ blastonères l et d'un seul blastomère $k$, qui, à son tour, se transformera en $l$ sans se di-

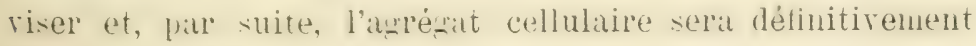
formé de 89 blastomères, qui se trouveront tous arricés à la phase limite $l$ de l'érolution de l'oeuf.

Nous rogons donc que, lans le déreloppenent inonodique, l'oeuf subit une différenciation des la première division, et, si 
des deux hastomeres 7 et ", dérives de la première cytodiérèse, nous considérons l'un d'eux, par exemple b, comme re-

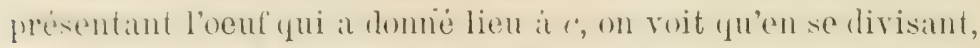
l'vente" n'al pas seulement produit une autre cellule dillérente, mais qu'il s'est, lui aussi, quelque peu modifié. On roit encore que les deux phases, dont l'une est lil plus arancée et l'autre la moins arancée dans l'érolution, ne sont représentées que par une seule cellule, tandis que les phases intermédiaires le sont toujours par plusieurs cellules; que, la division progressant, une cellule, la plus arancée, atteint, la première, la phase limite de la potentialité irolutire-de l'oeuf et, par conséquent, la diflérenciation histologique qui la caractérise; qu'enfin, si d'autres causes n'interviennent pas pour modifier le phénomène, toutes les autres cellules arrivent i cette même phase de l'érolution, ce qui fait que la différenciation histologique susdite ne sera plus représentée par un seul mais par plusieurs éléments. En outre, nous voyons encore que l'hètérogénéité des cellules constituant l'agrégat cellulaire, va toujour's s'accroissant jusqu'it l'apparition du premier blastomère de la phase limite, mais que, a partir de ce moment, elle ra au contraire en diminuant, jusqu'i l'instant oi l'agregat cellulatre anral alteint une homogéneité complete, puisqu'il ne sera plus constitué que des cellules à la phase limite,

Tout cela, évidemment, n'aura lieu qu'ì ces deux conditions:

$1^{\circ}$ ) que les substances dentoplasmatiques $x$, quoique diminuées, ne soient pas complètement épuisées;

$2^{\circ}$ ) que d'autres causes n'interriennent pas pour modifier l'accomplissement de l'évolution.

Mais si les substances deutoplasmatiques se sont épuisées, apros la fi" division par exemple, toute exolution ulterienre sera naturellement arrêtée, et l'agrégat cellulaire sera formé d'un certain nombre de cellules dans des phases diverses de l'évolution de l'oeuf, ainsi que nous l'arons démontré. Cet agrégat prourrat néanmoins constituer un organisme, c'est-it-dire une 
colonie d'individus différents, lesquels, par leurs fonctions vitales, pourront entretenir lit vir de tonte la colonie. Peut-itres avons-nous des exemples de colonies semblables dans quelques-mus de ces oryanismes infëriemes, dont les individus manifestent évilemment des différences flus on moins marquées.

si nous consilérons que tous les ornanismes puricellubires ne sont que des colonies de cellules vivant en symbiose, c'estit-dire des colonies où les diflérents indiridus s'entr'aident réciproquement (car les substances sécrétées par certaines cellules peuvent servir de nutrition a d'autres) et que la complexité plus ou moins grande de ce système symbiotique est dependante de l'hétérogénéité des individus qui le constituent, nous pourons facilement admettre que l'agrégat cellulaire issu de la segmentation de l'oeuf puisse former un système symbiotique et, par conséquent, un ormanisme pluricellulaire, quoique d'une très grande simplicité.

Nous arons jusqu'ici supposé que, pendant l'évolution de l'oeuf, il n'intervient pas d'autres causes capables de la modifier et nous arons vu quels en sont les résultats. Supposons maintenint le cas opposé et eximninons quelies conséquences en découlent. Il est évident qu'il n'y a pas d'autres cas possibles.

Supposons donc que, pendant l'évolution de l'oeuf, il puisse se former - par quel mode? nous le rerrons plus tard - des substances spéciales chimiques capables d'entrer en réaction arec le hioplasma de certaines cellules le lougregat cellubare.

Nous avons vu, par exemple, que, dans l'agrégat que l'on vient de considérer, les cellules $l$ ne peuvent plus suirre une évolution ultérieure, parce qu'elles ont atteint la phase limite de la potentialité évolutive de l'oeuf.

Mais si les substances nourelles, que nous indiquerons par $x_{1}$, peurent serrir de nutrition à ces cellules, alor's leur bioplasma pourra assimiler et arriver jusqu'au dédoublement biomoléculaire et, par suite, à la cytodiérèse. Le blastomère $l$ se divisera donc en deux autres cellules; et si nous supposons que le développement soit toujours hétérogénétique, on pourra 
représenter les nourelles cellules issues de la division de $l$ par $b_{1}, c_{1}$.

Or, si les substances $x_{1}$ ne sont pas aptes, par leur nature sprediale, a réagir arec les hioplatsmats des cellules $l_{1}, c_{1}$, celles-ci ne progresseront pak ultériemrement et s'arriteront dans leur évolution à ces deux phases. Par conséquent, après la $6^{\circ}$ dirision, aussitit que d'autres cellules a la phase $l$ apparaîtront dans l'agrégat cellukire, elles aussi, en assinilant les substances $x_{1}$, se diviseront à leur tour en $b_{1}$, $c_{1}$. Le nombre des cellules $b_{1}, c_{1}$, de la nouvelle lignée ira donc s'accroissant à chatyue thatse de l'érolution de l'oeuf, et cet accroissement surritera seulement lorsque s'arretera lit production des cellules I ou bien lor'sque les substances a seront épuisées. L'itgrézalt cellulaire, après cette nourelle prolifëration de cellules, sera done constitué d'autant de cellules $\eta_{1}$ et d'autant de cellules $c_{1}$ qu'il $y$ avait auparavant de cellules $l$, c'est-a-dire de 89 cellules $b_{1}$ et 89 cellules $c_{1}$, en total 178 cellules, dont aucune ne se troure plus dans les phases caractéristiques de l'érolution de l'oenff, mais dans les phases spéciales $7_{1}, c_{1}$. L'apparition des nouvelles substances de nutrition or a done détomrne les cellules / de la voie sur latquelle elles se trouraient anpararant, et qui représentait la phase limite de la potentialitie de l'oendip et a placé les cellules de lat nouvelle lignée datns une atutre direction suivant laquelle elles pourront arriver à une diflérenciation histologique qui sera éridemment différente de $l$.

En effet, nous arons supposé tout à l'heure que les substances $x_{1}$ ne sont pas aptes à réagir ultérieurement avec les cellules $b_{1}, c_{1}$. Mais si nous supposons, au contraire, ainsi que l'arons fitit pour l'oenf', que ces memes substances puiscent encore servir de nutrition mon senlement a ces nouvelles cellules, matis encore anx antres qui en deriveront apres leur division, toutes ces cellules, sous l'action de ces substances, fourront suive une simpe le transformations successives, dont lit nature sera dejendante, arant tout, de la constitution chi- 
mique du bioplasma des cellules /, leur point de répart, et puis de la constitution chimique les substances ${ }_{1}$. Le bioplasma $l$ possèdera donc, en présence de ces substances, une potentialité érolutive, tout comme l'oeuf, lui aussi, possistait aupararant une potentialité ívolutive résultant des constitutions de son bioplasmat et des substances deutoplatsmatiques a.

Nous pourons donc supposer que, ces conditions étant réitlisées, les hastomeres l possèrlent, en présence des substances $r_{1}$, une potentialité, qui peut les amener par des transformattions et divisions successires, jusyu'it une phase quelcompue, par exemple it la phase $l_{1}$, phase linite de cette potentialité; et, si le déreloppenent de ces blastomeres est toujours munoulique, anisi que je le suppose toujours pour les raisons que j’ai déjic exposées, on obtiendra, par la division de chaque blastomère $b_{1}$ une cellule $c_{1}$ et une autre $c_{1}$, et par la dirision de chaque blastomère $c_{1}$ une cellule $d_{1}$ et une autre cellule $e_{1}$.

De cette manière, le nombre des cellules de la nouvelle ligree ra saceroissant très rapilement au fur et a mesure que celles-ci s'approchent de la phase linite /, de leur potentialité érolutive. En mème temps, les phases caractéristiques de la potentialité de l'veul disparaitront peu it peu, a mesure que les antres cellules furogressernt dans la nourelle direction de leur érolution, et l'agrérat cellulare deviendra naturellement toujours plus riche en cellules.

Arrivées it la phase limite $l_{1}$ les cellules auront acquis une constitution bioplasmatique et, par consinuent, une differenciation histologique déterninée par la nature des changements subis pendant leur érolution: mais, a cette phase, elles s'arriteront éridenment, et, peu it peu, par des divisions successives, les autres cellules, qui se trourent dius des phises moins avancées, progresseront vers la phase $l_{1}$ et $y$ arriveront successivement. L'agrégat cellulaire résultera alors constitué de nombrenses cellules, et, plus précisément, de $89 \times 89=7921$ cellules posiélant la constitution bioplasmatique caractérisant la phase $l_{1}$. 
Tout celat, irilemment, aura lien si nous supposons que les substances in ne solent pas épuisées at que les cellules $I_{1}$ ne puissent pas trourer, dans le milieu ambiant, d'autres substances aptes a les nourrir. Mais si l'on suppose que de nourelles substances $x_{2}$ apparaissent dans ce milieu et que, par len constitution, elles puissent servir a l'assimilation des cellules $l_{1}$, celles-ci se diriseront en deux autres cellules, et de cette manière, on ohtiendra une troisieme lignée de cellules, qui, i leur tour, pourront pusséder une potentialité évolutive, capable de les porter, par des transformations et divisions sllcessives, jusqu'it une phase limite nourelle $l_{2}$, toujours en suirant le développement monodique.

Après cette troisième lignée de cellules, nous pourons, d'atprès le même raisonnement, en supposer une $4^{\text {e }}$, une $5^{\mathrm{e}} \ldots$ une ne, et les cellules pourront ainsi arriver à des différenciations histologiques très éloignées de la constitution de l'oeuf, leur point de départ; mais, pour arriver à ces différenciations, elles seront forcées de passer par les phases intermédiaires de leur évolution.

Tout ce que je viens de dire pour les blastomères ? de la $l^{\text {e }}$ lignée, pour les cellules $l_{1}, l_{2}$ etc. de la $2^{\circ}$ et $3^{\circ}$ lignée peut être appliqué aussi aux autres cellules précédant la phase $l$. Nous pourons supposer, par exemple, que les nouvelles substances nourrisantes, a a lieu d'agir sur les cellules l, puissent agir sur les cellules $f, g, h, i$ en provoquant un développenent monolique diférent de celui quelles auraient suivi par la simple action des substances deutoplasmatiques $x$. Le résultat sera analogue it celui que nous arons obtenu en considerant le blastomere l. Les cellules calpibles de réarir avec les substancess nourelles seront létournées de leur évolution primitive pour en suivre une autre, dont la nature sera tou-

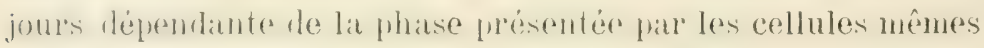
et de la constitution chimique des substances qui ont provoqué la déviation de leur évolution. 
De tout ce qui précede on peut donc conclure:

$1^{\circ} P a r$ le dérelomiement monorlique, les cellules dérivées de la segmentation de l'oenf atteignent tres rapidement la phase limite de la potentialité érolutire de l'oenf.

$2^{\circ} P(\eta)$ ce mode de déreloppement, toutes les cellules issues re la division surient une même direction vers la phase limile, el, si elles ne sont jas détominées de lewr direction pai dirutres causes, arirent toutes it cette phase.

30 Toute différenciation histologique, quelle que soit sa nature, ne sera done pas représentée par un seul, nais yar jusieurs éléments.

4. Dans l'agrégat cellulaire iésultand de la segnentation de l'oeuf, suirant le déreloppement monodique, il y a toujours une cellule plus arancée et une autie cellule moins arancée que les auties dans l'érolution.

$5^{\circ}$ La segnentation de l'oeuf produit dans les cellules de l'agrégat une hétèrogénète qui ra toujour's croissant jusqu'a l'apparition de la phase limite. A jartir de ce moment, l'hetèrogéneité peut diminuer jusqu'a disparâtre complètement.

$6^{\circ}$ A chaque division de la cellule la plus arancée, l'oeuf progiesse de deux phuses dans son érohution; it chaque division de la cellule la inoins arancée, l'oeuf alundonne une phase minitive de son érolution.

7o Si, pendant la segmentation de l'oeuf, des substances nourissantes nouvelles peuvent agir sur les cellules de l'agrégat dans une phase quelconque de leur érolution, celles-ci seront détournées de lent direction pirinitire el suivont une aulie érolution, qui les amènera it une différenciation chimique dependant de la phase it laquelle elles se trouvaient an moment de lew déviation el de la nature des substances qui ont provoqué celle-ci. 


\section{Chapitre VII.}

\section{L'asynchronisme de segmentation.}

SOMMAIRE : Impossibilité du synchronismo parfait dans la division des blastomères - Causes de cotte impossibilité - La durée de la période assimilatrice - La duréo de la période cytodiórésique - L'asynchronismo accéléré et l'asynclironismo ralenti - Effets de l'asynchronismo accéléré ot du développement monodique - La segmentation de l'oeuf' - La production des sillons - Le rythme de segmentation - La polarité et la symétrio bilatérale de l'agrégat cellulaire - Les cellules homonymes contemporaines - L'accélération de la segmentation générale - L'asynchronisme ralenti et ses effets - Le ralentissement de la segmentation générale - Résumé.

Afin de rembe parlititenent compréhensible lat théorie du développement monodique je n'ai pas voulu tenir compte, dans le chapitre précédent, de deux phénomènes qui accompatgnent la segmentation de l'oeuf', it satroir: l'asynchronisme le segmentation et la position réciproque des cellules. Il est donc nécessaire de les considérel maintenant, d'autant plus que ces deux phénomènes, et particulièrement le premier, ont me grande importance dans l'ontogénèse, importance qui n’a lais îé jusqu'ici reconnue et qui, je l'espère, ressortira dans toute sa valeur, d'après les considérations qui vont suivre.

En examinant le développement monodique, j’ai toujours supposé, pour plus de simplicité, que les divisions des différents blastomeres de l'agrégat cellulaire avaient lien simultanément. Mais il faut se demander maintenant si cette simultanitis est posibible ou du moins probable. Nous savons rue, lank lo développenent hétérogénétique, l'oenl' se rlivise en deux cellules difrérentes l'une de l'autre et différentes de l'veuf. res denx cellules so diviseront-elles simultanément ou bien dans des temps différents? 
Nous pourons domner ì cette question une réponse rationnelle en examinant attentivement les camses intimes qui provounent la division, ef que nous connaissons déjit d'apres l'exposé de la première partie de cet ouvrage.

On sait que le phénómène de la cytodiérèse ne peut pas commencer sans la division préalable des biomores du bioplasma. On sait aussi que la division des biomores ne peut se faire sans le dédoublement des biomolécules qui les constituent, et que le dérloublement des bionolécules exige, le la part de celles-ci, l'assimilation d'atomes nouveaux. Or cette assimilation, nous le sarons, consiste dans une série de réactions chimiques plus ou moins complexes, reations dont le nombre et la nature sont étroitement dépendants de la constitution des biomolécules d'une part, de la constitution des substances nourrissantes d'autre part, et enfin des conditions physiques dans lesquelles ces réactions s'accomplissent.

C'est une notion tout à fait élémentaire que les conditions physiques ont une grande influence sur la rapidité l'accomplissement les phénomènes chiniques. L'̉lectricité, la lumière et surtout la chaleur exercent sur les réactions chimiques une action très énergique en les provoquant, ou en les ralentissant, ou bien encore en les accélérant suivant le degré de leur intensité. Mais il est érident que, si l'on suppose que les comlitions physiques sont toujour's les mèmes, la rapilité ou Ia lenteur des réactions ne dépend que de la constitution chimique des substances réagissantes. Or, dans le cas que nous devons examiner, l'oeuf et les cellules qui dérivent de sil sermentation se trouvent dans les memes conditions physiques, ou, du moins, nous n'arons pas de raisons plausibles mor supposer que ces cellules, formant par leur ensemble un agrégat cellulaire très petit, puissent se trouver dans les conditions différentes. La durée de la période assimilatrice de ces cellules, c'est-it-dire des réactions successives de leur assimilation, ne sera donc dépendante que de la nature de leur binplasmat et le lat constitution des substances reuto- 
plasmatiques $x$. Nais comme ces dernieres sont, elles aussi, toujour's les mèmes dans toutes les cellules de l'agrégat (car nous avons supposé l'oeuf isotrope) il est évident que la durée de la périorle assimilatrice des diflërentes cellules ne dépendra que de la constitution de leur bioplasma.

Si donc la période assimilatrice du bioplasma $a$ de l'oeuf a une certaine durée, il est très probable que la durée de la période assimilatrice de la cellule $b$, ne sera pas la même, mais ou plus longue ou plus courte. De même, les durées des périodes assimilatrices des cellules $c, d$, $e$, etc. seront différentes entre elles et différentes de celles de $a$ et $b$, et la différence de durée entre ces périodes sera, très probablement, d'antant plus grande que la différence de constitution chimique entre les bioplasmas de ces diverses cellules est, elle aussi, plus grande. Nous pourrons même, par cette considération, juger de la diflérence hoplasmatique entre deux blastomères, différence qui échappe à nos moyens de recherche, d'après la différence entre les durées de leurs périodes assimilatrices. C'est dire que si deux cellules, daus les mêmes conditions physiques et se nourrissant des mêmes substances, accomplissent leur périorle assimilatrice dans des temps peu différents, il est très probable que leur's bioplasmas ne. sont que peu différents. Si, au contraire, leur's périodes assimilatrices ont une durée très diflérente, c'est que, probablement, lemrs hioplasmats possedent les constitutions chimiques, elles aussi, très différentes.

Supposons donc, pour mieux concréter nos idées, qu'un oeuf a ait été fecondé à minuit et que sa période assimilatrice soit de la durée de 2 heures. Aussitot que le bioplasma du spermatozoide s'est uni avec le bioplasma ovulaire et que les

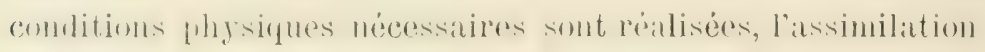
commencera. (Je me réserve de démontrer, dans une autre partie de ce travail, pourquoi, dans la plupart des oeufs, l'assimilation ne peut s’accomplir qu'après la pénétration du spermatuzode). A 2 hanes, les phénomenes de lassimilation se- 
ront achérés, les biomolécules et puis les hiomores subiront leur division et commencera la cytodiérèse. Alors nous verrons apparaitre le premier sillon de segmentation.

Ici, comme pour tous les autres sillons qui paraîtront plus tard, il faut tenir compte de certains phénomènes secondaires qui ne modifient pas substantiellement les résultats, mais qui, dans l'observation des faits réels, peuvent altérer, apparenment du moins, la segmentation.

On sait que, d'après mon interprétation, la cytodiérèse n'est que l'effet de l'orientation des biomores constituant le bioplasma, c'est-it-dire le noyau et le cytoplasma de la cellule. Or, cette orientation ne peut pas évidemment commencer a la périphérie, mais à l'intérieur de la cellule et doit s'étendre peu à peu jusqu'a la périphérie. Nous savons encore que, si la cellule contient des substances brutes, telles que les substances deutoplasmatiques, et si lo bioplasma, dans sa division, contracte arec les particules brutes des rapports de position, ces substances sont entrainées passivenent dans la division. Il s'ensuit donc que, du commencement de la cytodiérèse, c'est-à-dire de l'orientation des biomores du noyau et des astrosphères jusqu'a la division complète, s'écoulera un certain laps de temps nécessaire pour l'orientation progressive des autres biomores du bioplasma arec les particules brutes; et co laps de temps sera naturellement d'autant plus long que les particules brutes sont plus nombreuses, c'est-a-dire que le deutoplasma est plus abondant.

Par conséquent, si l'on observe la segmentation de l'oeuf, non pas d'après l'apparition des figures caractéristiques de la cytodiérèse, mais d'après l'apparition à l'extérieur des sillons de segmentation, on verra apparaitre ces sillons plus tard, et ce retard dans la formation des sillons à la surface de l'oeut sera d'autant plus fort que les substances dentoplasmatiques seront plus abondantes.

C'est pour cela que dans les oeufs télolécites, ou l'accumulation du dentoplasma est plus grande a l'un de ses priles, les 
sillons sétendent ì ce poile toujours plus tard qu'au poile opposé.

Il pour'ra mème arriver, dans certains cas, que l'orientation soit déjá acherée il l'intérieur de la cellule avant que la division soit parfaitement accomplie à la surface de l'oeuf et, par suite, arant que les sillons soient complets.

Il s'ensuit que si, dans l'examen' de la segmentation des

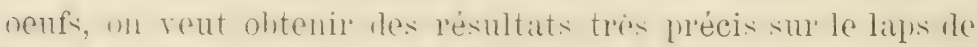
temps qui s̈écule antre les divisions des ditlërentes cellules, il faut calculer ces laps de temps d'après l'apparition d'une hes ligures callacteristiques de lat cytorliérese. par exemple, le la plaque équatoriale.

Revenons maintenant à notre exemple. L'oeuf, à 2 heures, commence donc sal premiere division. Celle-ci exige naturellenent, far alle-minne et indépenulamment des substances deutphasmatiques, un certain laps de temps pour s'acherer: car elle ent nne orientittion, et, par conséquent, un phénomène qui s'accomplit peu à peu. Mais afin de rendre plus simple

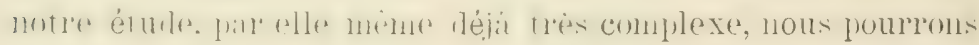
négliger ce laps de temps et supposer, théoriquement bien entendu, que la durée le la période de la cytodiérèse soit nulle. Celiı, d'ailleur's, ne modifiera pas les résultats auxquels on arrivera.

De la première dirision de l'oeuf $a$, résultent donc deux cellules $b$, c, dont les périodes assimilatrices, pour les raisons

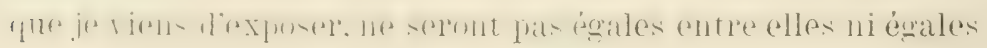
¿ celle de l'oeuf.' Elles seront donc plus longues ou plus conrtes.

Supposons-les plus courtes, et, plus précisément, supposons que la périorle assimilatrice de 7 soit de 5 minutes plus courte que celle de $a$, et la périorle assimilatrice de $c$ de 5 minutes plus courte que celle de 7 . La périole assimilatrice de $b$ aura ronc une rurée de 1 heure 55 minutes; la période assimilatrice de $c$, une durée de 1 heure 50 minutes.

I.e lecteur comprendra facilenent que ce n'est lá qu'une 
simple supposition pour mieux concréter nos idées. Dans la réalité des faits naturels, les difrérences entre les périodes

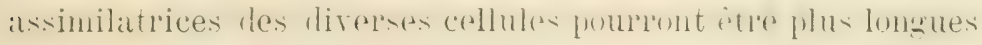
ou plus courtes que je ne viens de le supposer; mais le résultat final auquel on peut arriver ne changera pas substantiellement, ainsi que nous le rerrons plus tard.

De même, les différences de durée entre les direrses cel-

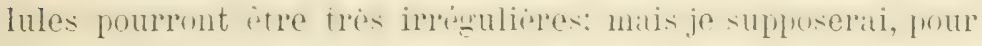
plus de simplicilé, qu'elles soient les mêmes, c'est-i-dire, qu'entre les durés les périnles assinilatrices des diverses phases: $a, b, c, d \ldots$ la différence soit toujours de 5 minutes.

Si done nous indiquons les phases de l'érolution de l'oeuf par des lettres, on aura:

pour la phase $a$, une durée de la période assimilatrice de $2 \mathrm{~h}$.

\begin{tabular}{|c|c|c|c|c|c|c|c|}
\hline$\gg$ & $\gg$ & l) & $\gg$ & $»$ & 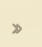 & $\gg$ & 1 h. $5 \bar{s}^{\prime}$ \\
\hline 》 & $\gg$ & $c$ & $\gg$ & $\gg$ & $\gg$ & $\gg$ & $1 \mathrm{~h} .50^{\prime}$ \\
\hline » & $\gg$ & l & $\gg$ & $\gg$ & $\gg$ & $\gg$ & 1 h. $45^{\prime}$ \\
\hline 》 & $\gg$ & $e$ & $\gg$ & $\gg$ & $\gg$ & $\gg$ & 1 h. $40^{\prime}$ \\
\hline$\gg$ & $\gg$ & $f$ & $\gg$ & $\gg$ & $\gg$ & $\gg$ & 1 h. $35^{\prime}$ \\
\hline$\gg$ & $\gg$ & ! & $\gg$ & $\gg$ & $\gg$ & $\gg$ & 1 h. $30^{\prime}$ \\
\hline » & $»$ & $m$ & $\gg$ & $\gg$ & $\gg$ & $\gg$ & 1 h. $25^{\prime}$ \\
\hline$\gg$ & $\gg$ & $i$ & . & $\gg$ & $\gg$ & $\gg$ & $1 \mathrm{~h} .20^{\prime}$ \\
\hline$\gg$ & $\gg$ & li & $»$ & $\gg$ & $\gg$ & $»$ & 1 h. $15^{\prime}$ \\
\hline$\gg$ & $\gg$ & $l$ & $\gg$ & $\gg$ & $\gg$ & $\gg$ & $1 \mathrm{~h} .10^{\prime}$ \\
\hline$\gg$ & $\gg$ & $m e$ & $\gg$ & $\gg$ & $\gg$ & $\gg$ & 1 h. 5 \\
\hline » & $\gg$ & $n$ & $\gg$ & $\gg$ & $\gg$ & $\gg$ & $1 \mathrm{~h}$. \\
\hline 》 & $\gg$ & 0 & $»$ & » & $\gg$ & $\gg$ & 0 h. วัว \\
\hline
\end{tabular}

Nous supposerons que la phase limite de l'érolution soit $p$. Cela étant posé, concrétons encore mieux nos idées par des figures schématiques dans lesquelles l'veuf est représenté par un cercle, les plans de division rerticaux par des droites rayonnantes, et les plans horizontaux par d'autres cercles plus petits. Ians ces figures, afin que l'on puisse voir l'oeuf entier, je le suppose étalé sur un plan et vu d'en haut, de 
manicre que le pòle supérieur se trouve au centre du cercle "1e prile inférienr a sa circontërence. Les cellules dérivées les sil sementation yni se troureraient nomalement an pole inférieur, nous les verrons donc à la périphérie, ce qui naturellenent prorluira dans tout l'agrégat une déformation, qui d'ailleurs n'a pas d'importance dans nos considérations.

D'autre part, nous le verrons sous peu, ces figures auront larantage de nous donner une explication de certains phénomènes qui ont ité observés dins less segnentations des oenfs et qui sont restés, jusqu'ici, inexpliqués.

Enfin, j’ajouterai encore une observation.

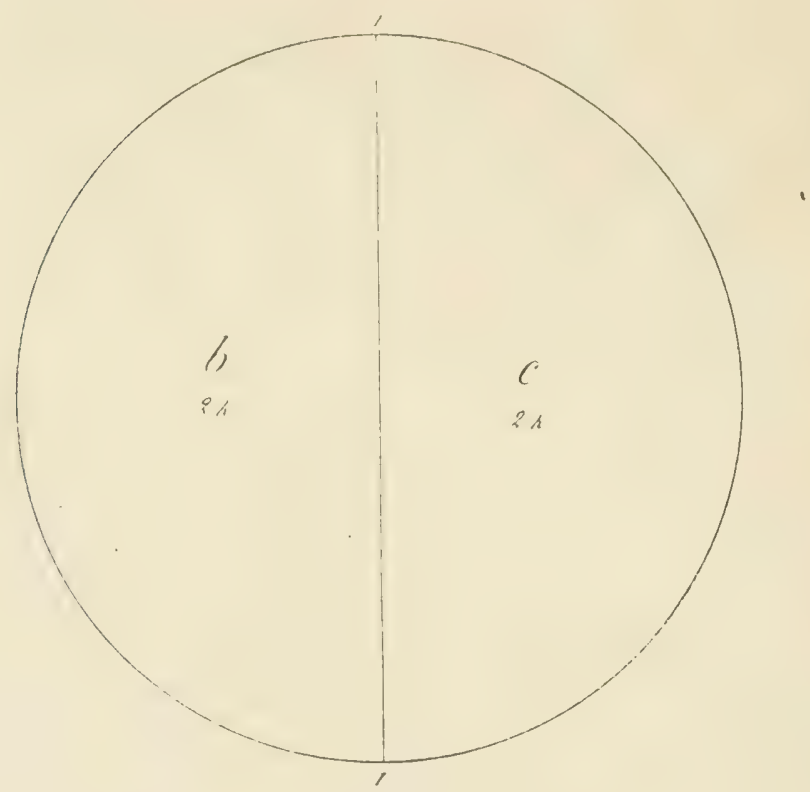

Fig. 1.

J'ai démontré, dans la $1^{\mathrm{e}}$ partie de ce travail, que les directions des plans de division ne sont dépendantes que des causes mécaniques extérieures à la cellule. J'ai aussi démontré qu'à cause de la division, les diflérentes cellules d'un 
atgrégat cellulaire peurent subir des déplacennents. Tont (n) les confirmant, je ne reviendrai pas sur ces questions; mais je ferai seulement remarquer que, dans les figures qui ront suivre, je ne tiens pas compte de ces déplacements ni de lat direction réelle des plans de division. Ainsi qu'on pourra le roir apres l'exposition de mon interprétation de l'ontogéneseses, la direction des plans de segmentation n'a pats, dams cer phenomène, l'importance que l'on y attache guénéralenent. Noms pourons done, sinon la négliger complètenent, la consilérer du moins comme un phénomène d'importance secondaire.

Donc, à 2 heures, a lieu la première division de l'oeuf a

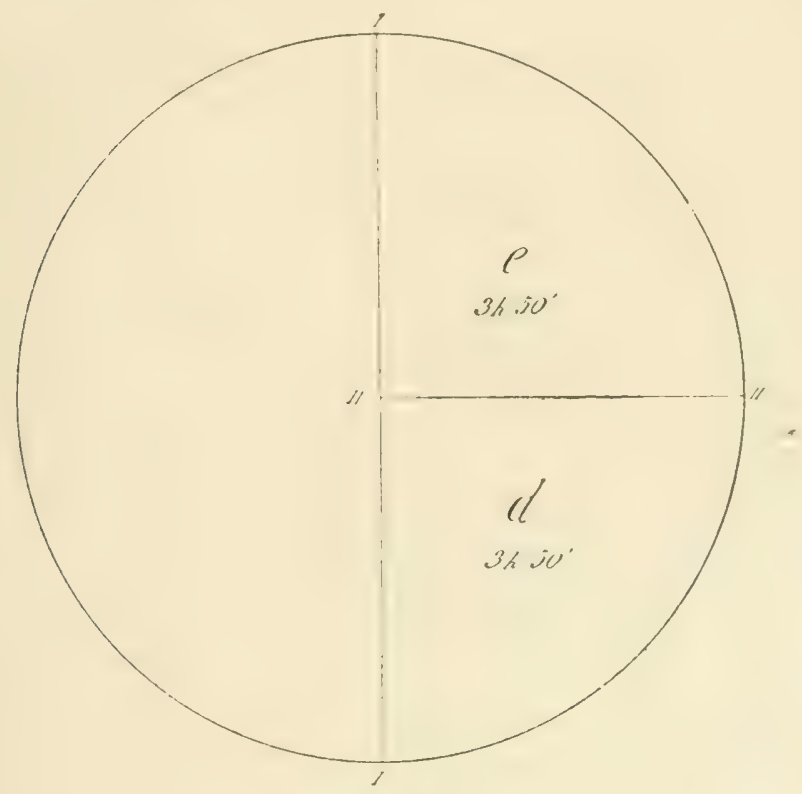

Fig. 2.

et de sa division naissent les deux cellules 7 , ( (figr. 1). Si l'oeuf est alécithe, le sillon apparaitra à peu près daus le même temps à sa surface; sí, au contraire, il est télolécithe le sillon apparatrat arant tout an prile supérieur, et puis nous le verrons sétendre peu à peu rers le póle inférieur qu'il 
atteindra si, le deutoplasma nétant pas trop abondant, la division est totale.

A 3 h. 50', le blastomère $c$ se divisera (fig. 2); mais le blastomère $b$ ne se divisera qu'il 3 h. $55^{\prime}$ (fig. 3). Par conséquent, le $\underset{\sim}{2}$ sillon de segmentation apparaîtra, avant tout, dans un des deux blastomères et s'étendra peu ì peu à l'autre. Mais, conme l'atcomplissement de la cytodiérèse exige un certain lizps de tempse évilemment plus long que 5 minutes, lit division du hlastonère l) commencera bien avant que celle du

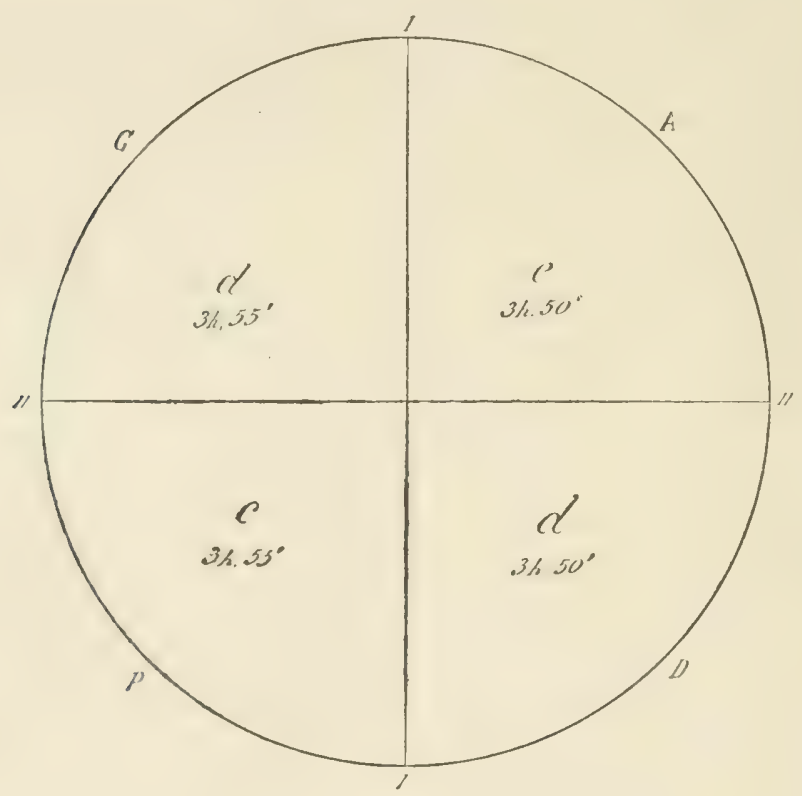

Fig. 3 .

blastomère $c$ soit acherée. Dans l'observation des faits réels, il nous paraîtra done que les deux divisions sont synchroniques, parce que nous verrons les blastomères tous les deux dans une des phases de la cytodiérèse: ce qui n'est pas précisement exact. Si d'ailleurs on observe la segmentation d'un oeuf télolécithe, on verra le sillon commencer dans un des 


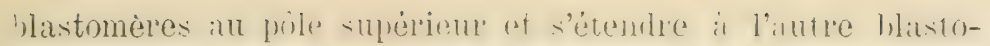

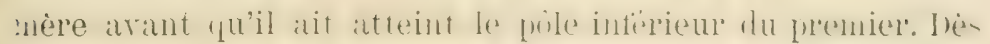
ce moment, il faut remarquer que, dans l'agrégat de 4 cellules, il y a une cellule $e$ la plus arancée, qui est née arant la cellule $c$ la plus arriérée dans l'évolution, et que les deux celiules $d$, qui se trouvent ‘ la même phase, n’ont dans leur âge qu'une différence de 5 minutes.

A 5 h. 30', le blastomère $e$ se dirisera, et nous relrons apparatre dans un des 1 blatstoneres le III" sillon de semgnen-

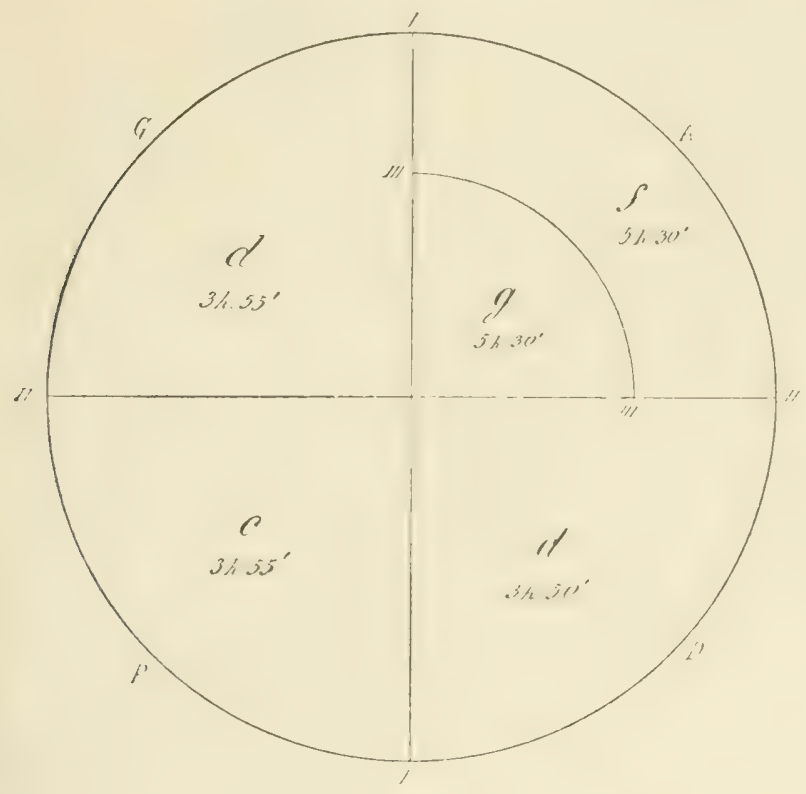

Fig. 4 .

tation (III, fig. 4). A 5 h. 35’, se rivisera le blastomère d né it $3 \mathrm{~h} .50^{\prime}$, et $5^{\prime}$ plus tard, e'est-it-dire a $5 \mathrm{~h}$. 40', se dirisera

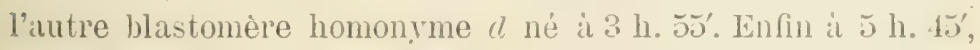
se divisera aussi le blastomère $c$. Par conséquent, le III ${ }^{\circ}$ sillon ne se produira pas au même moment dans tous les quatre blastomères, mais il apparaitra dans l'un d'eux et puis s'étendra peu à peu aus autres (fig. 5). 
Ici encore, pour les memes raisons exposées à l'égard du I" sillon, si l'orientation qui produit la cytodiérèse a une durée plus longue que lo minutes, le blastomère c commen-

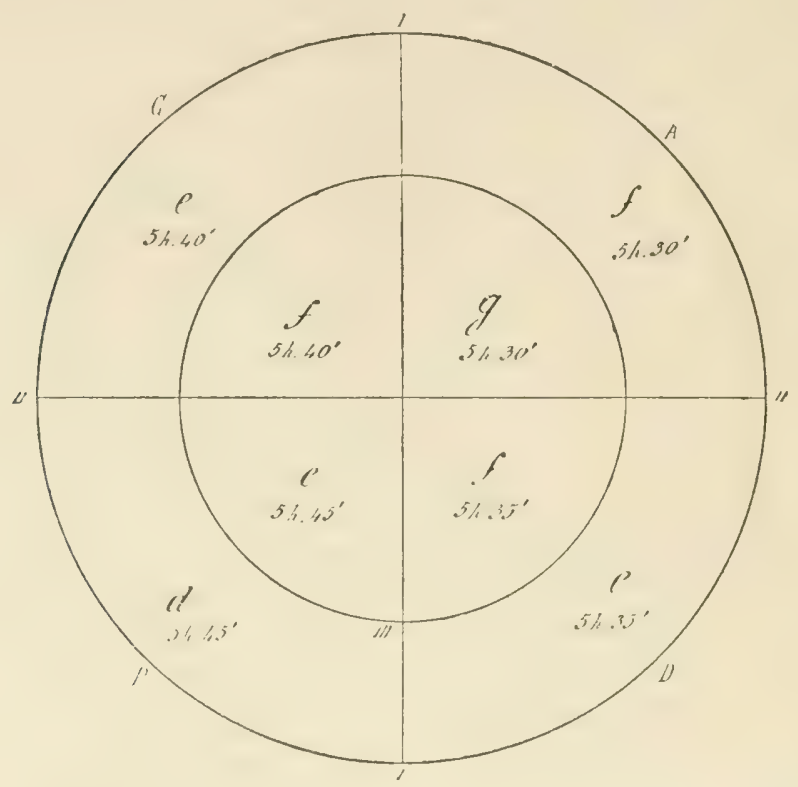

Fig. 5.

cera sa dirision arant que celle du blastomère $e$ soit acherée. Parl conséquent, nous rerrons encore les 1 hlastomères se diviser presque simultamément. cependint, ce synchronisme ne nous paraîtra pas aussi érident que dans le cas précédent; car nous rerrons les bastomeres tous les quatre en division, mais éridemment dalls des phases diverses de la cytodiérèse.

A 7 h., $g$ se divisera, et apparaîtra le IVo sillon de segmentation (IV, fig. 6), qui commencera au pôle supérieur et s’étendra du même côté vers le pôle inférieur; car à 7 h. 5', se divisera le blastomère $f$ né à $5 \mathrm{~h}$. 30'. Ce même sillon s'étendra aussi de l'autre côté, mais plus tard, à 7 h. 25 ', c'esta-dire lorsque $e$, né à 5 h. 45', se divisera à son tour. 
Cependant, avant que le IVe sillon ait atteint le pồle inférieur de ce lernier coté, et plus précisément a T h. 10', c'està-dire 10 minutes seulement après le $I V^{r}$ sillon, le $V^{e}$ sillon

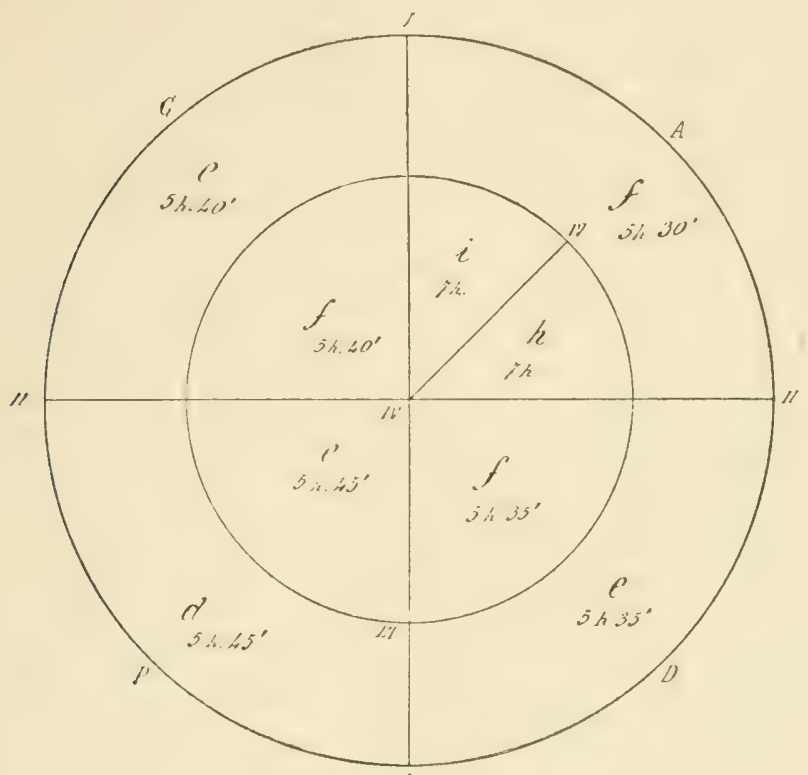

Firg. 6.

aura fait son apparition au pôle supérieur: car le blastomère $f$ : né à $5 \mathrm{~h}$. 35', se divisera ( $\mathrm{r}$. fig. 7 ), et ce sillon s'é-

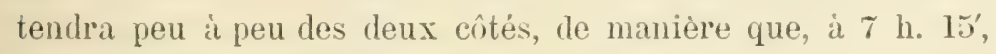
(fig. 8) il aura atteint le pôle inférieur d'un côté et se sera rapproché de ce pôle de l'autre côté, tandis que le IVe sillon qui est apparu avant le $V^{\circ}$ ne sera arrivé au pôle inférieur que d'un côté seulement.

Enfin à $7 \mathrm{~h}$. 20, le Ve sillon aura atteint le pôle inférieur de l'autre côté; à 7 h. 25', le lVe sillon commencera à s'étendre, lui aussi, de l'autre côté et à 7 h. 30', il sera arrivé au poile inférieur. I.es IVe et V" sillons de sementation seront ainsi acherés (fig. 9). 


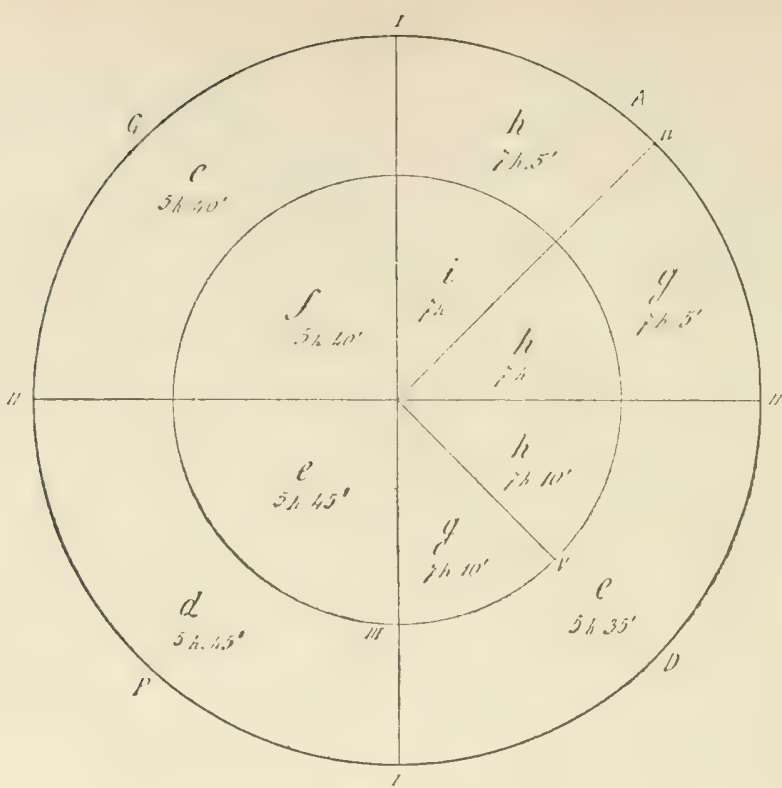

Fig. 7.

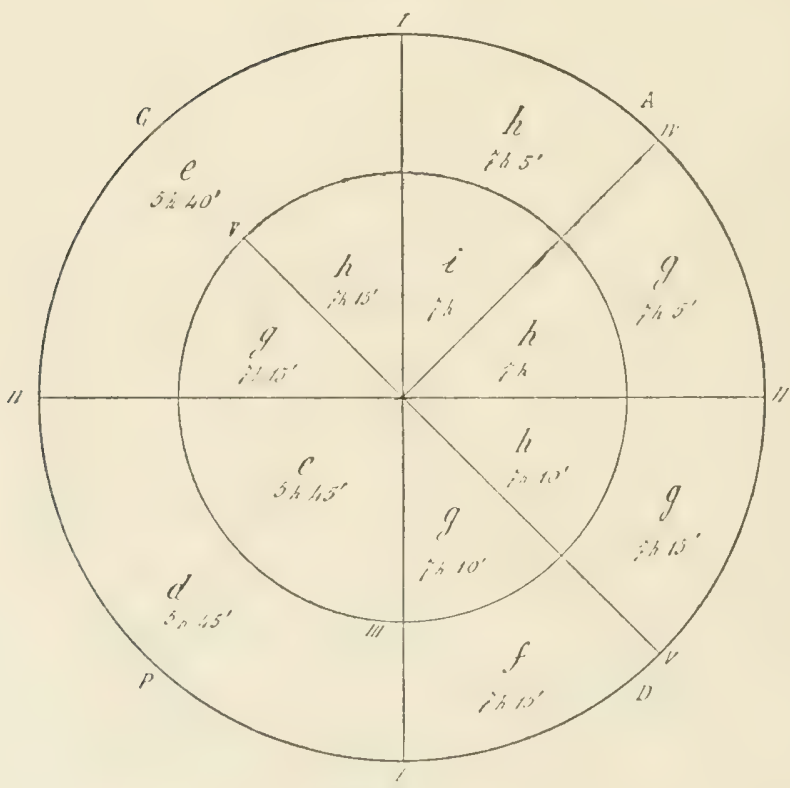

Figr. 8. 
Or, si l'on suppose que l'orientation des biomores et, par' conséquent, toute la période de la cytoliériese ait me durée de :3) minutes, l'asynchronisme de segnentation commencerat à se révéler assez évidemment dans la formation des IVe et

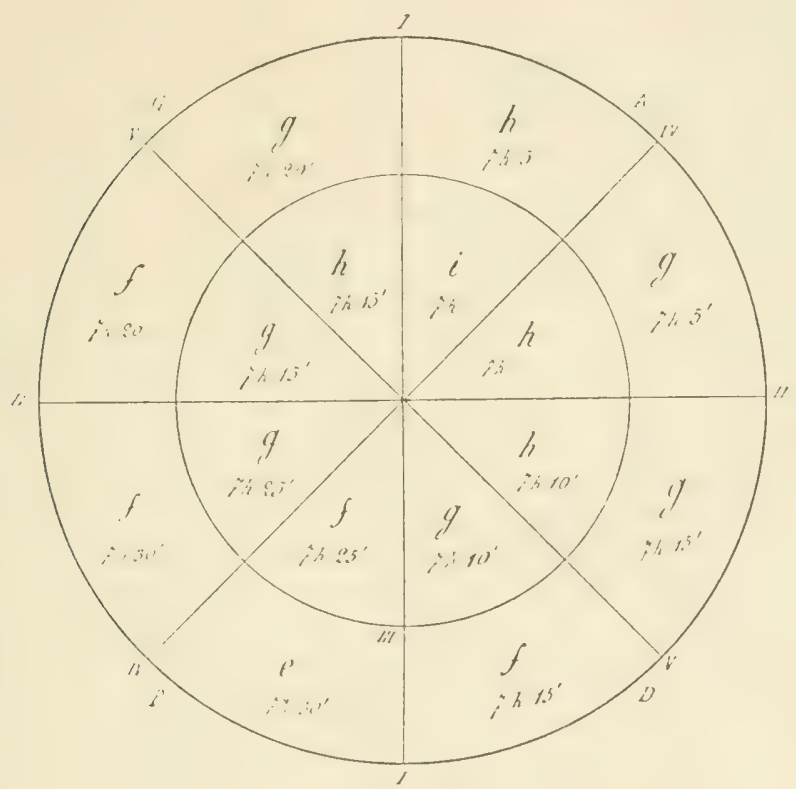

Fin. 9.

Ve sillons. En effet, lorsque nous rerrons apparaître les premières phases de la division, dans le blastomère $d$, le blastomixe g se serat complétement livisé, et les blastomères f se trouveront dans une des dernirres phatses de la cytoliérese.

A partir de ce moment, l'asynchronisme nous apparaitra toujour's plus évident; car les blastomères ne se diviseront plus simultanément, mais nous verrons leurs cytodiérèses s'accomplir cà et là dans l'agrégat cellulaire, apparemment sans ordre, en réalité dans un orilre déterminé par les durées de leurs périodes assimilatrices. C'est ce que les figures suivantes démontreront. 
Le lecteur qui comniat le mode de segmentation des oeufs de certains animaux, tels que les grenouilles, les tritons etc. ne pourra fare a moins de remarquer entre cette marche thémique de lat segmentation et colle des latits réels me anatlonge tres frappante. Iess ambryologistes qui ont ètudié ninutiensement la marche de la sementation daus ces oeufs ont remarqué, sans pouroir en donner une explication, cette inregularite dans la production des sillons, irregularité que nous pourons maintenant expliquer très facilement par l'asynchronisme de segmentation, dont je riens d'exposer les caluses.

D'ailleur's, cet astrnchronisme de segmentation est un phénomène constant dans tous les oenfs, mème dans ceux de certains animax, par exemple les Coelentérés, ou l'on croit généralenent que le srnchronisme est parfait. s'il en ètait ainsi, on derrait roir les cellules de segmentation de ces oeufs, se diviser toujours sinultanément à une phase quelconque de la regmentation, ce qui n'arrive pas. On sait 'n efret que les divisions sont presique synchroniques, ou, du moins, qu'elles parraissent telles an premieres phases de la segmentation, mais que ce synchronisme disparait, a mesure que la segmentation frongesse. Lal cause de ce sinchronisme apparent est due à ce fait, que la diffërence entre les lureses les périodes alssimilatrices des ditlërentes phases de lérolution de l'veuf est

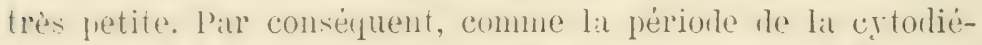
rese a mue durce beanconp plus longue que cette diflërence, il s'ensuit qu'aux premières phases de la segmentation, les cellules commenceront lenr dirision it des temps dillërents, mais bien atrant qu'elle soit atcherée dans quelques-unes d'elles. En examinant lanrégat cellulare, noms rerrons donc toutes ces cellules simultanément dans quelques phases de la cytodiérèse; mais, en réalité, ces phases ne seront pas exactement les mêmes. C'est seulement à des phases ultérieures de la segmentation, que l'asynchronisme pourra se réréler, c'esta-nlire lorsque la ditrérence entre les durées des périodes as- 


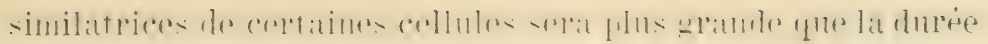
de la période cytodiérésique.

A \& h. $20^{\prime}$, c'est-i-dire $10^{\prime}$ seulement après l'achèvement du IVe sillon, commence le VIe, parce que le blastomère $i$, né à

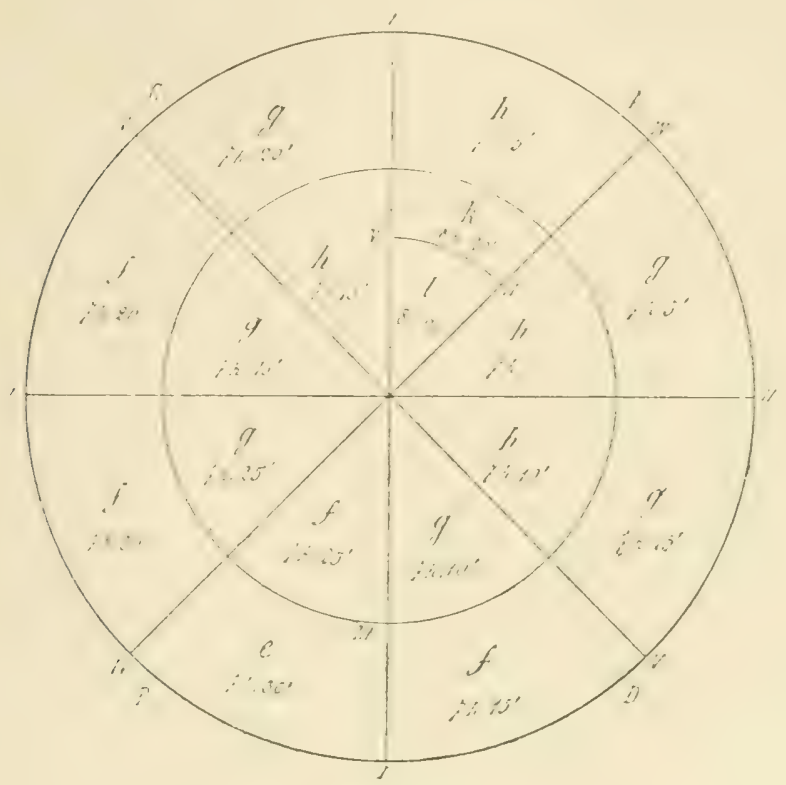

Fig. 10.

$7 \mathrm{~h}$., se divise à son tour (VI. fig. 10). La segmentation ra

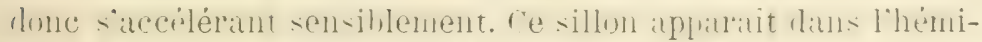

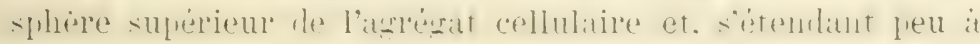
peu aux autres blastomères, it droite et it grauche, il sera com-

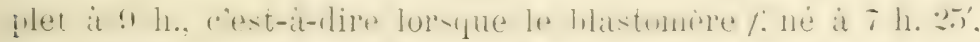
qui est le moins arancé de tous les blastomères supérieurs, arrivera, lui aussi, a se diviser (fig. 11).

Mais bien arant que ce sillon soit complet, a $8 \mathrm{~h}$. 30', paraitra le VII sillon, qui, lui aussi, s'étendra peu à peu à tous

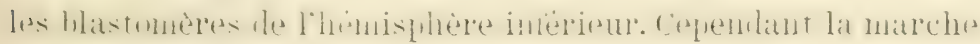
de ce sillon sera plus lente; car à $9 \mathrm{~h}$, elle ne sera pas encore 
acherée (fig. 11), et ne s'achèvera qu'à o h. 10', lorsque se dirisera le blastomère $e$, né ì 7 h. $30^{\prime}$.

I () h. 30', c'est-it-dire 20 minutes seulement après l'achèvement du VIIo sillon, le blastomère $l$, né à 8 h. 20 , se divisera et, par suite, apparaîtra le VII $\mathrm{I}^{\circ}$ sillon, au pôle supérieur. Ce

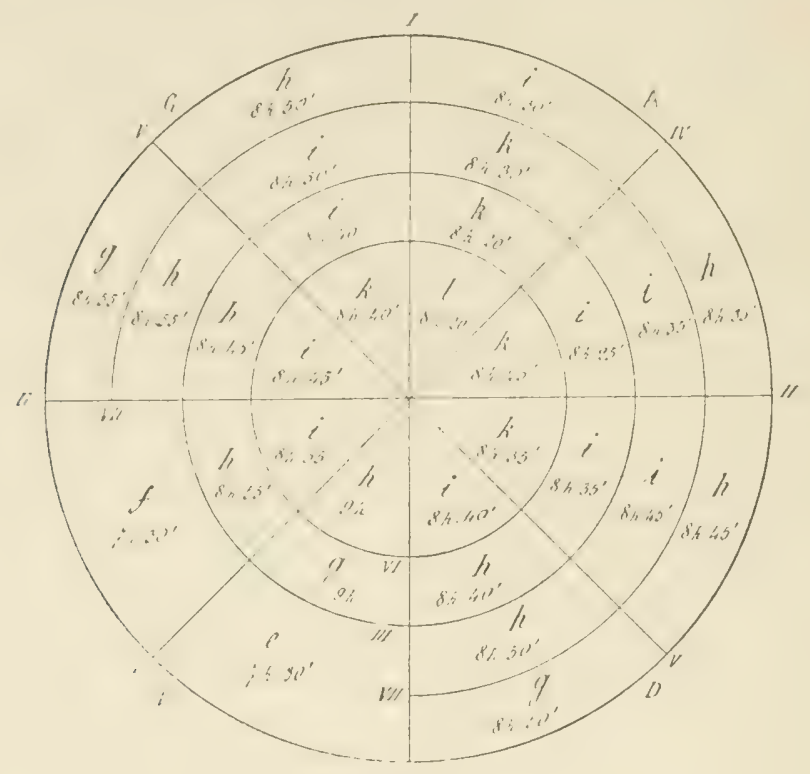

Fig. 11.

sillon s'étendra du pôle supérieur au pôle inférieur; mais a () h. 40, paraitra le IX, parce que se divisera le blastomère $k$, né ¿̇ 8 h. 25', et $10^{\prime}$ après, c'est-it-dire à 9) h. 50' se divisant $k$ né à 8 h. 35', apparaitra le $\mathrm{X}^{\mathrm{c}}$ sillon (fig. 12) et puis à 9 h. 55', se divisant $k$ né a 8 h. 40, se produira aussi le XIe. Ces quatre sillons rayonnants du pòle supérieur marcheront vers le pôle inférieur. Le VIII sillon marchera assez vite vers le póle inférieur; car à 9 h. $50^{\prime}$, et, par suite, $20^{\prime}$ seulement après sa première apparition, le blastomère $i$, né à $8 \mathrm{~h} .30^{\prime}$ se divisera a son tour, et le VIII sillon aura donc atteint le pòle inférieur de ce còté; au contraire, sa marche du côté opposé sera 
très lente, parce que le blastomère $\int$ né à 9 h. $10^{\prime}$ ne se dirisera qu'a 10 h. 45'. La division de ce blastomère complètera le VIIIe sillon.

Mais avant meme que ce sillon soit complet, à $10 \mathrm{~h}$. 30', la cellule $n$, née à 9 h. 30', se divisera et ainsi, tout près du pòle

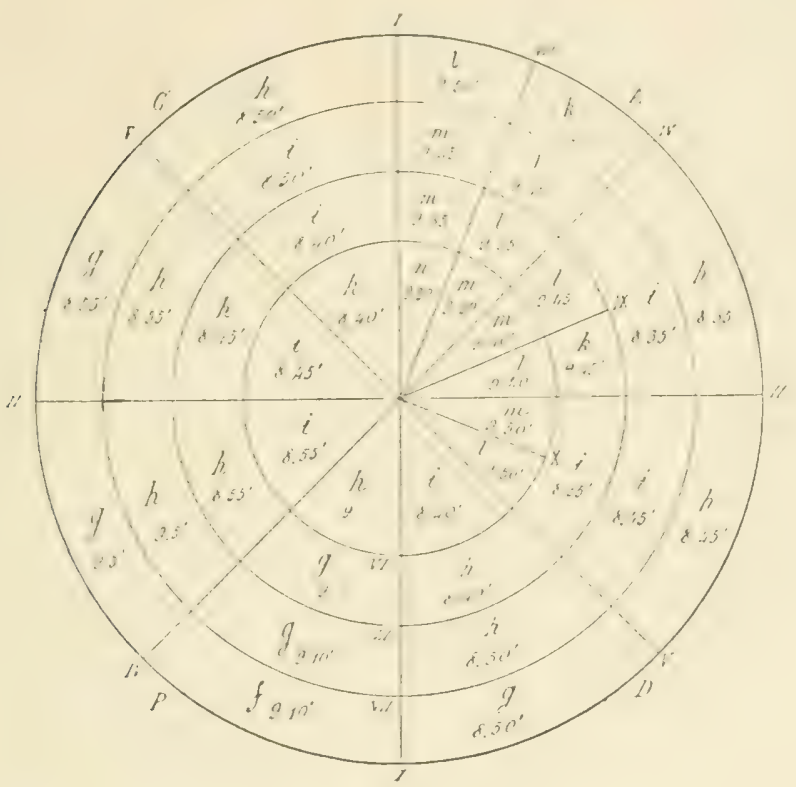

Fig. 12.

supérieur; paraitra le XII ${ }^{\circ}$ sillon. La segmentation, ainsi qu'on le roit, va toujours s'accélérant, particulièrement au pỏle supérieur.

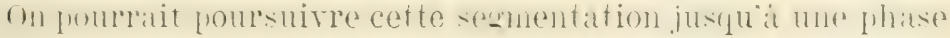

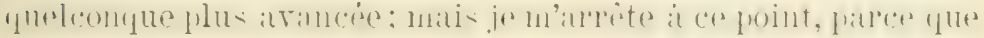

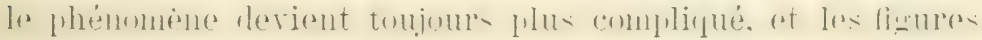
perdraient de leur clarté. D'ailleur's, ces phases de segmen-

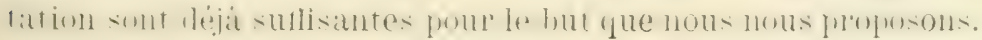

Examinons donc les conséquences qui découlent de l'asynchronisme de segmentation. 
Dés la II segmentation, l'agrégat cellulaire qui en résulte présente 4 cellules, dont deux $d$ se trouvent dans une mème phase de l'évolution de l'oeuf, et deux autres $c$, $e$, dans des phates dillërentes. Or', les deux cellules homonymes a sont presque contemporaines, parce que leurs igges ne different que

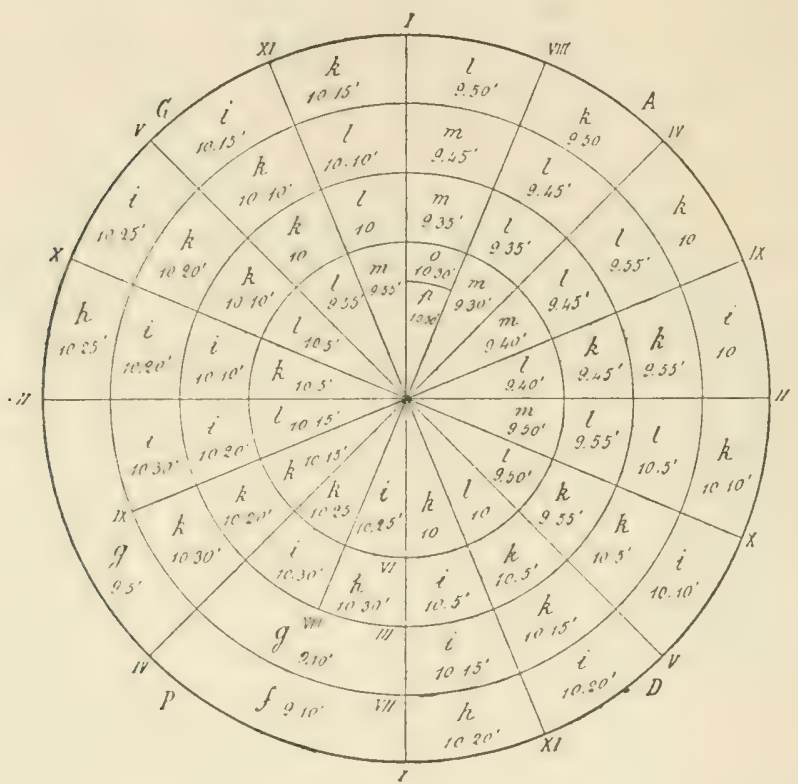

Fig. 13.

de 5 minutes. La cellule $e$, qui se trouve à une phase plus arancée de l'évolution et qui se diviser'a plus tòt, est née arant la cellule e, laquelle, an contraire, se trouve dans la phase moins avancée. A ce moment, les deux phases extrêmes $c, e$, ne sont séparées l'une de l'autre que par la seule phase intermérliaire $r$.

A la troisième segmentation, le blastomère plus avancé est $\therefore$ la phase $g$, tandis que le plus arriéré est encore à la phase $\therefore$ Entre les phases $c$ et $g$, il y a maintenant trois phases intermédiaires: $d, e, f$ (fig. 4). 


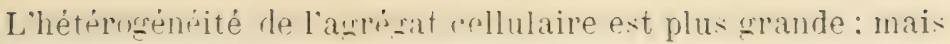
les blastumères ef reprosutant les phases intermediaires se trouvent dius: tous les t qualrants de lagrégat. Cependant il

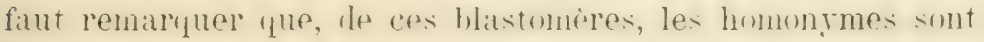
tous d'un àge différent, et, plus précisément, que, des trois blastomères f plus arancés dans l'solution, le plus àre, né a 5 h. 30, se troure dans un quadrant et les deux autres plus jeunes daus deux autres qualdants. De mime, de trois hastomères $e$ moins arancés que filans l'évolution, le moins àgẻ se trouve dans un quadrant et les plus àgés dans deux autres.

Après le Ve sillon (fig. 9), les deux phases extrêmes $e$, $i$ sont séparées par les trois phases intermédiaires $f, g, h$; après le VIII sillon, les phases extrèmes $f, n$ le sont par les 6 phases intermédiaires $g, h, i, h, l$, $m$; et à $10 \mathrm{~h}$. $30^{\prime}$, les phases $f, l$, par 8 phases intermédiaires.

Nous arons vu au chapitre précédent que, mème en sup-

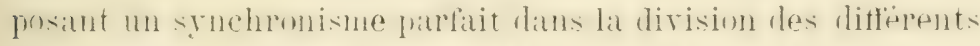

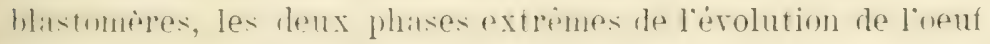
s'éloignent de plus en plus l'une de l'autre, à mesure que la spmentation [moresse. cet éloimentent progressif qui accé-

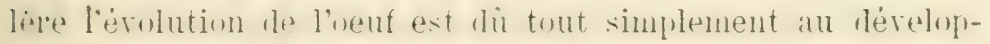
pement monodique; car, ainsi que nous l'arons ru, dans ce molr de déreloppenent, à chaune dirision des blastomères, loent progresse de leux phases lins son érolution, mais n'abandonne qu'une phase seulement.

l'asynchron isne desežnentation, et, plus précisément, l'asyn"hroni-me accéléré, tel flle moms renons de le comsilérer dins lexenple ci-rlevant expmes, en ajultant lone son action at relle du déreloppement monodique et en agissant dans le mème

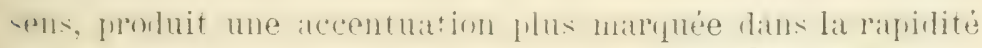
de l'érolution de l'oeuf et amène un des blastomères de l'agrégat cellulaire a la phase limite dans un temps plus court.

Dans notre exemple, une des cellules arrive a la phase $p$ a 10 h. 30', tandis que la cellule la moins arancée se trouve encore at la phase $f$. Cette cellule, à 10 h. 45', se dirisera a son 
tour pour donner lieu it deux cellules $/ 2, h$, dont la plus arrieree serat !. Or, en admettant que l'évolution de la cellule la mons arancer puisse se continuer tonjours régulierement, on peut démontrer qu'elle n'arriver'ait it la phase p' qu'it 20 h. 5', rest-it-1ire? h. 3.)' après l'aurivè de la cellule la plus arancée.

Ici il faut tenir compte que, pour plus de simplicité, nous avons supposi arrèté le développement it lo h. :3r', c'est-it-dire lorspue l'agrégat ne résulte constitur que de rie cellules. Mais. si l'on suppose que l'érolution puisse progressel' ultélien'enent, il est évident que, lorsine l'agrégat cellulares sera formé de quelyues centaines de cellules, la distance entre la cellule la moins avancée et la cellule lia plus avancée dans l'érolution seria beaucoup pius grande.

Si l'on examine la constitution de l'agrégat cellulaile a partir du moment où se produit le II ${ }^{e}$ sillon (figg. 3), jusqu'à sa dernière phase (fig. 13), on voit que toutes ses cellules sont contenues dans L quadrants . I, I', $D$, $(\dot{r}$, délimités par les sillons I, II, et que les cellules occupant le quadrant it ou bien représentent une phase de l'érolution de l'oeuf qui n'est pas représentée dans les autres quadrants, c'est-i-dire qu'elles n'ont pas leurs homonymes: par exemple, la cellule $g$ dans la fig. 5, la cellule $i$ dans la fig. 9, la cellule $l$ dans la fig. 11, les cellules $m, n$ dans la fig. 12 , et les cellules 0,1 dans la fig. 13; ou bien la phase qu'elles représentent est aussi représentée par d'autres cellules dans les autres quadrants, c'est-i-dire qu'elles possèrlent dans ceux-ci leur's homonymes: par exemple, la phase $f$ dans la fig. 5 , les phases $g, h$ dans la fig. 9, les phases $h, i, l$ dans la fig. 11 , les phases $i, k, l, m$ dans la fig. 13.

Cependant, si l'on compare les cellules du quadiant 1 avec les cellules homonymes des autres quadrants, on remarquera qu'elles sont toutes plus igrées que les autres. En effet, les cellules du quadrant 1 sont nées avant la naissance des cellules homongmes des autres qualdrants; par consérquent, elles soliviselont atant celles-ci, ot les phasess qu'elles representent 
disparaitront dams le qualrant I plus tit que dans les antres, de mème que les phases nourolles of plus atrancées de l'érolution feront leur première apparition dans ce mème quadrant $A$.

Une constitution analogue, mais en sens inverse, se retrouve dans le quadrant $P$. Fn eflet, dans celui-ci, sont contenues: $1^{\circ}$ ) la cellule la moins avancée daus l'évolution et qui n'a pas d'homonymes dans les autres quadrants; 20 ) d'autres cellules un peu plus avancées, mais qui possèdent leurs homonymes dinss les antres quarlrants. Cependant on remarquerat encore ici, que les cellules du qualrant $P^{P}$ sont toutes plus jeunes que les cellules lemm homonymes des antres quarlrants.

Enfin, si l'on examine les quadrants $D, G$, on constatera facilement qu’ils possèdent une constitution toujours identique, quelle que soit la phase de la segmentation que l'on veuille considérer, c'est-it-1ire qu’une phase queleonque de l'érolution de l'oeuf, représentée dans l'un de ces quadrants par une cellule, se troure ansi représentée dans l’antre par une autre cellule. En d'autres termes, toutes les cellules d'un de ces quadrants ont leurs homonymes dans l'autre. Mais ce qu'il faut remarquer surlout, c'est que ces cellules homonymes n'ont pas une différence d'àge très marquée, ainsi que nous l'avons vu pour les cellules homonymes des autres quadrants.

Au contraire, cette différence d'âge n'est que de 5 minutes, et elle ne sera janais plus grande que óninutes, quelle que soit la phase de la segmentation que l'on veuille considérer. Ces cellules sont donc non seulement homonymes, mais aussi contemporaines. Je les appellerai donc les cellules homonymes contemporaines. Ce n'est que dans ces deux quarlrants qu'on peut trouver de telles cellules.

Quelle est la cause de l'identité de constitution de ces deux quarrants et de la difrérence de à minutes dans l'àge de leurs cellules?

Nous la connaitrons facilement si nous remontons aux premières phases de la segmentation.

En effet, toutes les cellules des quardrants $D$ et $i$ derivent 
de la division des deux cellules primitives $d$ (fig. 3). Or, de ces deux cellules, l'une, celle du quarrant $D$, est née de la division du blastomère c et l'autre, celle du quadrant $G$, de la division du blastomère \%. Mais, comme nous avons supposé que la période assimilatrice de $c$ est de 5 minutes plus courte que la période assimilatrice de $b$, évidemment la cellule $l$ du quadrant $D$, dérirant de la division de e, naîtra ó minutes arant la cellule $d$ du quadrant $G$, laquelle dérivera de la division de 7 . Or, puisque ces deux cellules $d$ sont homonymes, tontes les cellules qui dériveront de leur division seront, elles aussi, homonymes, et, comme les cellules homonymes ont éridemment leur's périodes assimilatrices égales, les cellules issues de la segmentation des deux cellules a présenteront, rlans leur àge, la mème différence que les deux cellules primitives "l, e'est-ì-dire 5 minutes seulement, et cette différence ne changera jamais pendant toute la durée de la segmentation.

La différence dans l'àge des cellules homonymes contemporaines est donc dépendante de la différence de longueur' des périodes assimilatrices des deux premier's blastomères. Par conséquent, si l'on suppose que cette diffẻrence entre les périblos assimilatrices des deux premiers blastomeres est plus m moins grande, la difrérence dans l'igre des cellules homonymes sera, elle aussi, plus ou moins grande, mais, dans tous les cas, elle sera constante.

Au point de vue de la constitution des quadrants, on voit donc que l'agrégat cellulaire, it partir de la formation du $\mathrm{II}^{\circ}$ sillon, acquiert une polarité et une symétrie bilatérale, polarité et symétrie qui sont dues moins à la nature et à la position des cellules constituant les quadrants qu'à la diflérence de l'àge de ces cellules. Le caractère de la polarité dans l'agrégat cellulaire de la fig. 13 dérive de la présence dans le quarlrant $A$ des cellules $0, p$, et du fait que les autres cellules de ce quadrant sont toutes plus ìgées que leurs homonymes des autres quadrants; ou bien de la présence dans le 
quadrant p de la cellule $f$, et du fait que les autres cellules sont plus jeunes que leur's homonymes des autres quadrants.

Le caractère de la symétrie bilatérale résulte de la présence dans les deux quadrants $D$ et $G$ de cellules toutes homonymes entre elles; mais cette homonymie n'est que Mimportance secomtaire, barper que hes cellules homonymes existent aussi dans les autres quadrants. Ce qui donne a l'agrégat cellulaire la symétrie bilatérale, c'est surtout la contemporanéité des cellules homonymes.

Quelle est loririne de cette smétrie bilatérale! Lilpparition dans laugrétat collulatire des deux cellules d homon!nues et contemporaines après la formation du $\mathrm{II}^{\mathrm{e}}$ sillon.

Quelle est la cause de l'apparition de ces deux cellules? Le hérelopuenent nun whique. yni ast lit hase de mon interprétation de lontogénese.

En ellet, comme, dans ce monle de déreloppenent hétrogénetique, les denx cellules dérivant de la division d'une cellule mère relmésentent deux des philsas successires de l'érolution de roeuf, il sensuit nfressairenent, inéritablement, quaprèla livision des deux premiers blastmmires, deux des quatre cellules issues de leur segnentation se troureront dans la mème phase de lérolution, et la liffẻrence dans leur àre serat égale it la différence entre les durées des périodes assimilatrices des leux prenier: blastrmieres; en d'autres temes, elles seront homonymes et contemporaines.

Nous pouvons donc conclure que la cause de la symétrie bilitérale de l'agrégat cellulaire rémulant le lat segmentation he linsul est, tont simplenent, le léreloppenent monolique.

On pourrait m'objecter ici que la symétrie bilatérale de

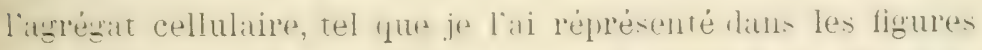
précerlentes, est la conséquencre non senlenuent du dérelop-

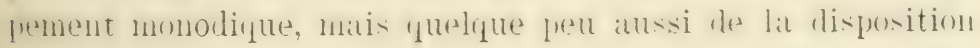
yu'ont les quatre blastomères $c$ d $d e$ dans la fig. 3; disposition qui est arbitraire et qui, par conséquent, pourrait bien être dillérente dans d'autres cas. Je peux démontrer très lacilement 
que, quelle que soit la disposition quon reuille supposer dans ces blastomères, le l'ésultat en est toujour's le même.

Il faut arant tout considérel que chacun des quatre blastomères $c d d e$ doit occuper une position dans l'espace.

Or, comme le deuximme plan de sermentation, soit à cause de la pression de la membrane vitelline des oeufs, lorsque celle-ci est présente, soit a callse de l'adhésion des blastontères, si la membrane ritelline manque, est toujours perpendiculaire, ou presque perpendiculare an fremiar plan, il s'ensuit nécessairement que les quatre blastomeres ne peurent aroir que les dispositions réciproques suivantes:

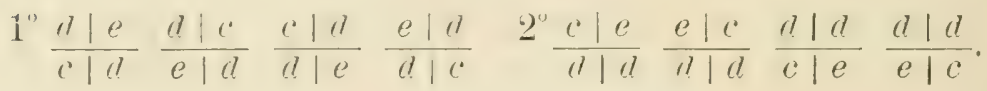

C"est dire que toutes les dispositions possibles se réduisent at deux types fondamentaux. Or, comme le premier de ces deux types est celui-là même que nous arons suiri dans la construction des figures précédentes, il ne nous reste maintenant à considérer que le deuxième.

Inus ce type, les deux blastomeres homonymes contemporains $d d$ occupent deux quadrants contigus, ainsi que les deux autres blastomères. Les cellules dérivées des blastomères d, c'est-it-dire les cellules homontumes contemporaines occuperont donc, elles aussi, deux quadrants contigus. Par conséunent, la stmétrie bilatérale de l'agrézat cellulaire ne sera pas effacée par ce noureau type de disposition.

Quant à la polarité, il est rrai que les deux blastomères 1. e qui lat diterminent et, par suite, toutes les cellules qui en rériveront, se trourent logées dans deux quadrants contigus, ce qui, de prime abord, paraît la détruire; mais si l'on consirlere que cette polarité n’est pas déterminér par les deux hastomeres at lat lisis, mais par un seul, on comprend facilement yutelle n'est gas du fout disparue dims re deuxieme type de disposition. I'atlleurs, nous anons matintes hois occasion, dans le cours de ce travail, de nous conranincre que la posi- 
tion réciproque res blastomères pst un cametere l'importance secondaire dans le développenent de l'ombryon et dans loorigine de sa symétrie.

Rerenons maintenant a l'asynchronisme de segrne+ntation.

Dans l'exemple que nous renons dis consilfirer, nous arons supposé que la diflerence entre lo durres les prériodes asimilatrices des diffërentes phases de l'arolution de l'veut est le 5 minutes, c'est-it-dire que cente ditlërence est tonjours constante pour toutes les phases. Nous arons ru, par les figzures précérlentes, que, dans ce can, les divisinns des cellules se succèrlent d'une manière spéciale hépendant précisément de cette diffërence. Lat succession de- livisions dims l'aurégat cellulaire constitue ce que nous fmurons appeler le, inthme te la segmentation. or, si l'on suppose que la différence entre les durées des périorles asinilatrices soit phus grande ou plus petite que J minutes, ou bien que cette ditlërence ne soit pas la mene pour toutes les phases, exilemment le rithme de la segmentation sera, lui ausi, différent, mais les conséquences finales ne changeront pas substantiellement.

Tous arons suppose jusquaci que lastnchronisme de sergmentation est accéléré, cest-d-dire qu'a mesure que l'oeuf progresse dans son érolution les durées des périorles assimilittrices des differentes phases sont graduellement plus courtes, et nous arons ru que, dans ce cas, la marche générale de la segmentation saccélère, que lat listance entre le blastonèrle le moins arancé et le hastomère le plus arancé dans létrolution saccroit de flus en plus et que les cellules dérivies de lit segmentation arrivent plus tit a la phase limite de lat prtentialité érolutive de l'oeuf.

Or, comme il n'y a que deux modes possibles d'asynchronisme, a saroir lastuchronisue accélére et latruchronisme

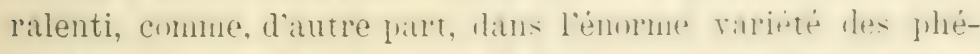
nomènes naturels biologiques, il fiatut consinlérer tom- les cats possibles, il ne mons reste maintenant it sximiner que l'astrochronisme ralenti, en supposant que les durées des périodes 
assimilatrices des phases de l'érolution soient graduellement plus longues.

Ici encore, afin de rendre parfaitement comparables les eflets de l'asynchronisme ralenti arec ceux que l'on vient d'obtenir par l'asýnchronisme accéléré, je supposerai done que loeut a possède une potentialité érolutive telle que, par les phases intermédiarres $b, c, d \ldots$. etc. il puisse arriver jusqu'à la phase limite $p$. Je supposerai de mime que la dillérence entre les durées des périodes assimilatrices des direrses phases de l'érolution soit de 5 minutes, c'est-i-dire que la période assimilatrice d'une phase quelconque soit de 5 minutes plus longue que celle de la phase précédente. Enfin, pour obtenir une comparaison parfaite arec les figures qui précèdent, je supposerai en outre que les blastomères les plus arancés occupent le pôle supérieur de l'agrégat cellulaire, c'est-ì-dire lil partie centrale des figures, et les blastomères les moins arancés le póle inférieur de l'veuf et, par conséquent, la périphérie des figures.

i.es durées des périodes assimilatrices des diflẻrentes phases de l'évolution seront donc les suivantes:

$$
\begin{aligned}
& \text { pour a. . . . . de. . . . } 0 \text { h. } 5 \mathrm{~s}^{\prime} \\
& \text {, l. . . . . . . . } 1 \mathrm{~h} \text {. } \\
& \text { "c...... . . . . . } 1 \mathrm{~h} .5 \\
& \text {, l. . . . . . . . . } 1 \mathrm{hl} .100^{\prime} \\
& " \text { e...... . . . . . . } 1 \text { 11. } 1.7 \\
& \text {, f. . . . . . . . } 111.90^{\prime} \\
& \text { } g \text {. . . . . . . . . } 1 \text { h. } 2 \mathrm{~s}^{\prime} \\
& \text { " h..... . . . . . } 1 \mathrm{~h} .30^{\prime}
\end{aligned}
$$

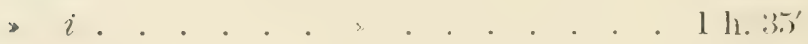

$$
\begin{aligned}
& \text { "k. . . . . . . . . . } 1 \text { h. } 10^{\prime} \\
& { }^{\circ} \text { l. . . . . . . . . l h. } 1 . \\
& \text { " } \\
& \gg n \text {. . . . . . . . . } 1 \text { ll. } 55^{\prime} \\
& \text {. o..... . ..... } 2 \mathrm{~h} \text {. }
\end{aligned}
$$


Cinquante-cinq minutes après la fécondation, paraitra donc le $\mathrm{I}^{\mathrm{e}}$ sillon, et l'oeuf $a$ donnera lien, par' sa division, aux deux blastomères b, $c$ (fig. 14).

Une heure aplès, e'est-it-lire it 1 h. Ju', se divisera le blastomère $b$, et puis, cinq minutes après, it 2 h., se divisera le

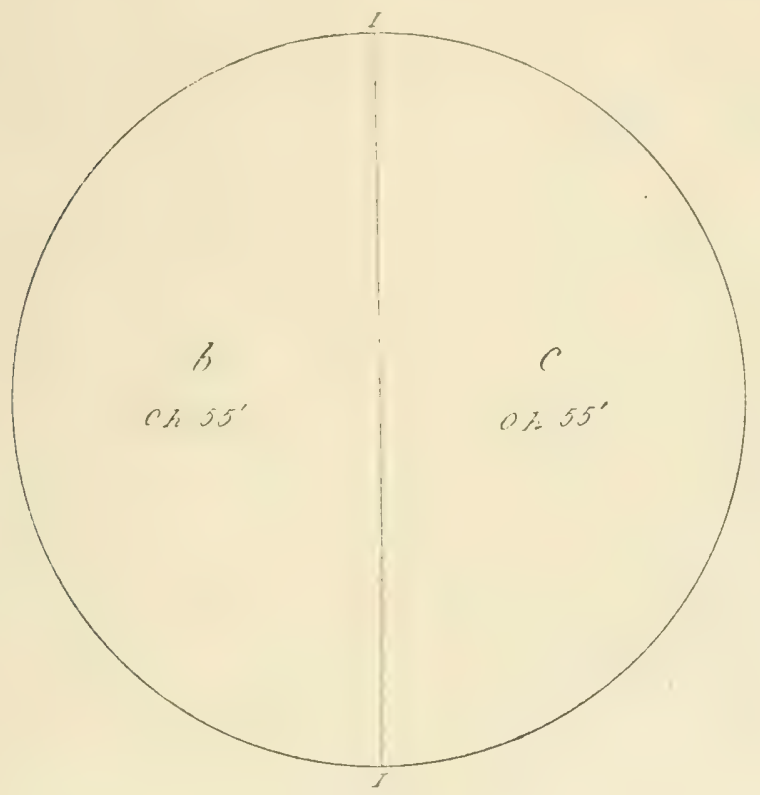

Fig. 14 .

blastomère $c$. Ainsi le II sillon sera complet et on obtiendra quatre blastomères $c, d, d, e$, parfaitement comme dans le cas de l'asynchronisme accéléré (fig. 3), sauf, bien entendu, l'ìge r'éciproque des cellules.

A 3 heures, paraîtra le III sillon dans le blastomère $c$ et il s'étendra peu ì peu, d'un côté et de l'autre, jusqu'au blastomère opposé e, qui ne se divisera qu'à 3 h. 1J'. Mais à 4 h. 10', le blastomère $d$, le moins arancé, se dirisera et, ainsi, commencera le IVe sillon (fig. 15). Avant que celui-ci soit complet, paraîtra le Vo sillon; et a 5 h. 25, lorsique les sillons IV et Ve seront achevés, commencera le VI sillon (fig. 16). 


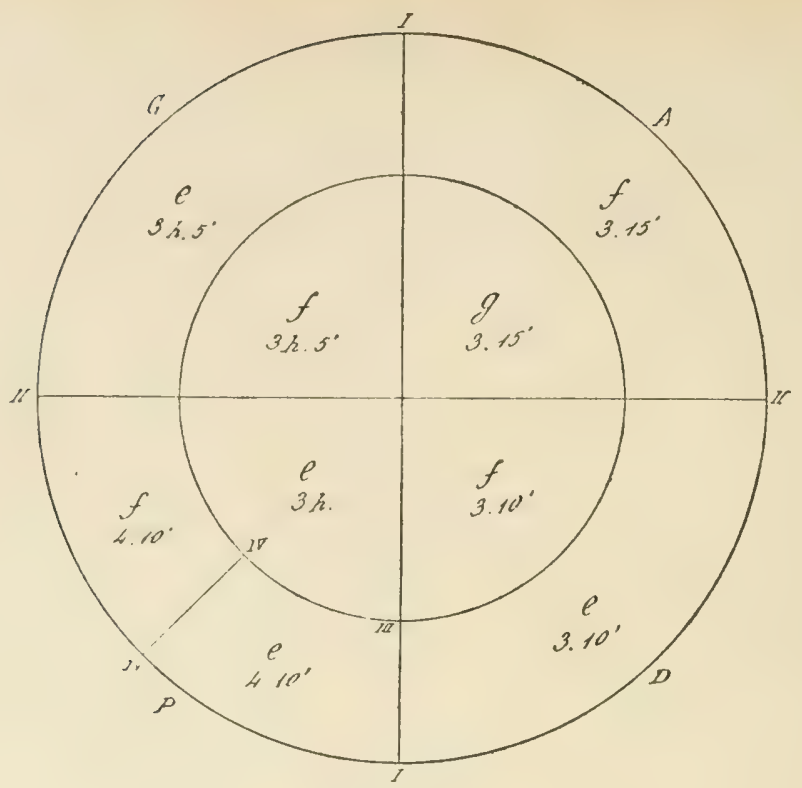

Fig. 15.

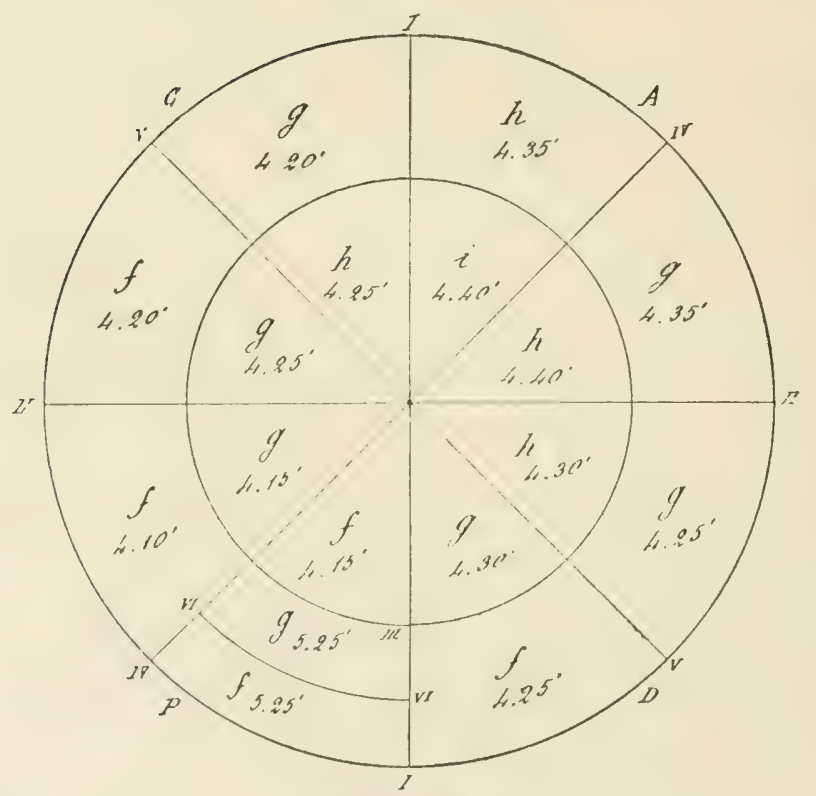

Fig. 16. 


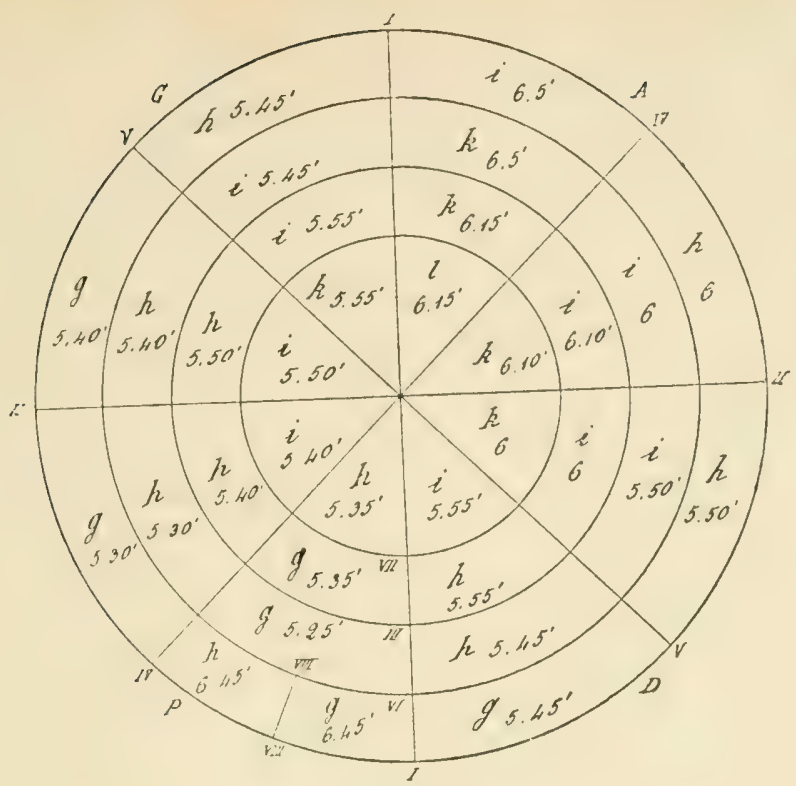

Fig. 17.

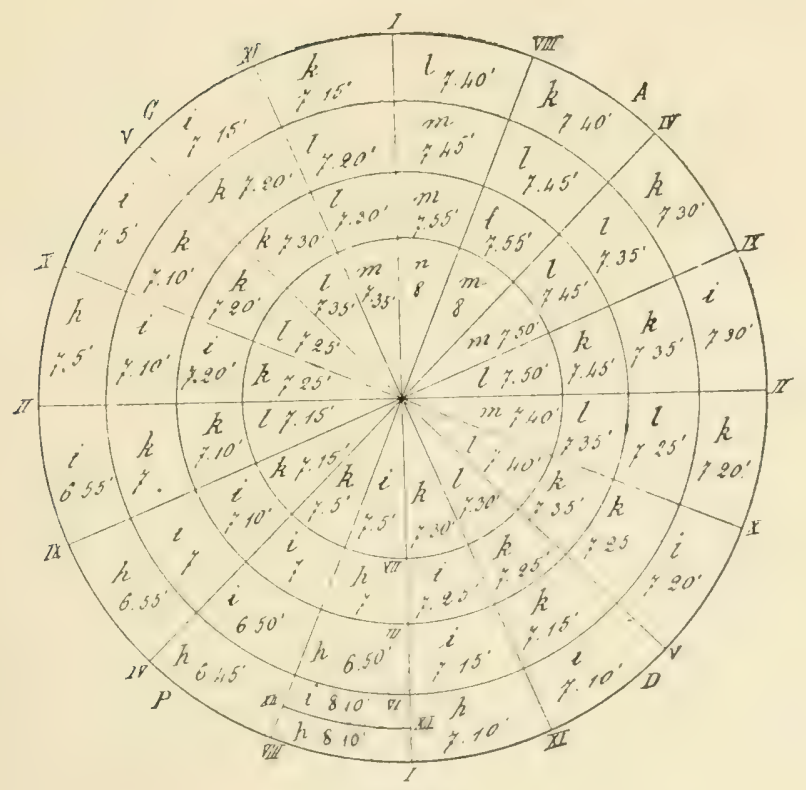

Fig. 18. 
A $6 \mathrm{~h}$. $45^{\prime}$, les T I et VII sillons étant complets, dans le blastomère f, le moins avancé, paraîtra le VIII sillon (fig. 17) et puis, successivement, les sillons $\mathrm{LX}^{\mathrm{e}}, \mathrm{X}^{\mathrm{e}}, \mathrm{XI}^{\mathrm{e}}$. Mais ceuX-ci, quoique parus plus tarl, se complèteront avant le VIII', qui ne serir acheré qu’i 7 h. $10^{\prime}$. A 8 h. 10', commencera le XII ${ }^{\circ}$ sillon. (fig. 18).

Celui ci s'étendra peu à peu d'un côté et de l'autre de l'agrégat cellulaire et ne sera complet qu'ì 9 h. 25'; mais, dans ce temps, paraitront aussi les XII ${ }^{e}$, IIVe et XVe sillons. A 9 h. $5 y^{\prime}$, le XVe sillon sera complet; mais $15^{\prime}$ minutes avant

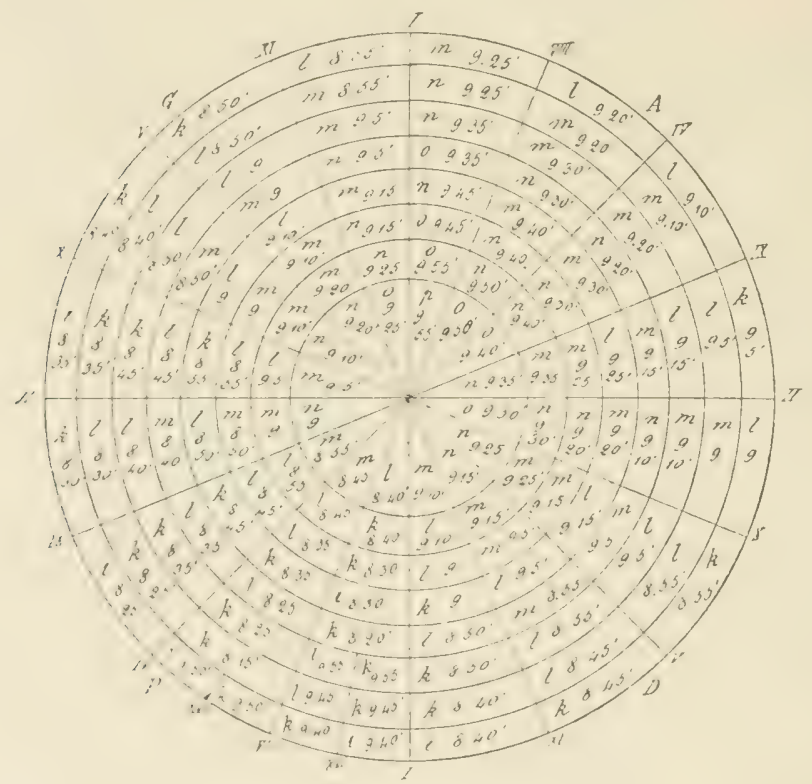

Fig. 19.

son achèrement, à 9 h. 40', aura par'u le XVIc sillon, suivi du XVII et du XVII (fig. 19).

A l'achèrement du XVc sillon, le blastomère le plus avancé a atteint la phase ple lérolution, c'est-it-dire la même phase qui, dans l'asynchronisme accéléré, a été atteinte par le commencement du XII sillon (fig. 13). 
Examinons maintenant ces figures (11-19) et comparons-les arec les fig. 1-1:3 de l'astrnchronisme accéléré.

Nous constaterons, arant toui, que le rythme de segmentation est le méme et que, jar conséquent, la marche de la segmentation est, elle aussi, la mème, abstraction faite, bien entendu, du lieu d'apparition des sillons. En eflet, dans l'asynchronisme accéléré, les sillons rerticaux, ainsi que nous l'arons vu, paraissent au pöle supérieur et s'étendent au pôle inférieur. Ici, au contraire, ces sillons suivent la marche inverse. Mais cela n'est dépendant que de la position que nous arons donnée aux blastomères dans nos fignures; car, si nous arions supposé les blastomères les plus arriérés situés au pòle supérieur et, par suite, au centre des figures, il est érident que la marche et le mode de segmentation ne seraient point différents dans les deux cas.

On constatera aussi que la constitution de l'agrégrat cellulaire est substantiellement la mème dans l'asynchronisme accéléré et dans l'astrnchronisme ralenti, c'est-i-dire que, dans tous les deux, elle présente une polarité et une symétrie bilatérale, symétrie dépendant de la présence, dans les deux quadrants $D, G$, des cellules homonymes contemporaines; polarité dépendant de la présence des cellules plus arancées dins le quadrant 4 , et des cellules moins arancées dans le quadrant $P$.

Cependant, si l'on fait un examen plus attentif de ces figures, (n) verra que, malgré cette analogie, elles présentent des difrérences qui, de prime abord, peurent paraitre sans importance, mais qui conduisent, au contraire, ì des conséquences très intiressantes pour le déreloppement ultérieur de l'embryon.

si l'on compare la fig. 13 arec la fig. 19, on rerra que, dans la première, la phase $p$ de l'érolution de l'oeuf a été atteinte par un des blastomères de l'agrégat cellulaire au commencement le la formation du XII sillon, c'est-ì-dire lorsque l'agrégat n'est constitué que de 62 cellules, tandis que, dans la seconde,

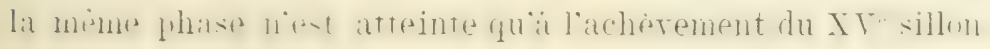




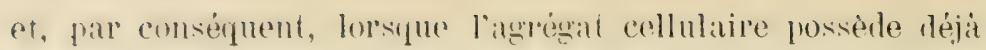
132 cellules.

On voit donc que, dans l'asynchronisme ralenti, il y a un retard dans l'évolution, quoique, dans notre cas, ce retard ne soit pas absolu mais seulement relatif.

En eflet, si nous considérons l'heure à laquelle la phase p) paraît dans les deux modes d'asynchronisme, nous voyons que, dans l'asynchronisme ralenti (fig. 19), cette phase parait a!) h. ns, tamdis que, dans l'asynchronisme accéléré (fig. 13), cette phase même ne parait qu’à 10 h. 30'. On dirait donc que, dans l'aspnchronisme ralenti, il y a une anticipation et non un retard dans l'évolution, ce qui est en contradiction arec ce que je viens de dire. Mais, en réalité, cette contradiction n'est qu'apparente; car si l'apparition de la phase $p$ se fait plus tot dans lasynchronisme ralenti, cela dépend de la différence entre less durées des périonles assimilatrices de l'oent, durees que nous arons supposies de 2 heures dans l'asynchronisme accéléré, et le jo minutes seulement dans l'asinchronisme ralenti.

Comparons maintenant les fig. 1-13 avec les fig. 14-19, et nous constaterons que, dans les deux séries, les effets de la segmentation sont à peu près les mèmes, it savoir qu'a mesure que celle-ci progresse, lat distance entro le blastomère le plus avancé et le blastomère le plus arriéré s'accroît de plus en plus, ce qui accentue la polurité de l'arrégat cellulare. Mais ce qu'il importe surtout de remarquer, e'est la constitution des quadrants de l'agrégat cellulaire dans ces deux séries.

Comparons la figure 9 arec la fig. 16 , et nous verrons que, dans toutes les deux, le blastomère le plus avancé $i$ du quadrant 1 n’est séparé du hlastomère le plus arancé g du quaIrant $P$ que par une seule phase intermédiaire h. Mais dans la fig. 9 , le blastomère $g$ du quarlrant $P$ étant né à $7 \mathrm{~h}$. 25', est plus jeune que le blastomère $i$ du quadrant $A$, qui est né à 7 heures; tandis que, dans la fig. 16, le blastomère $g$ du guadrant l' est plus agé que le blastomere $i$ du quadrant a, 
et, plus précisément, la diflërence ontre les ires de ces deux blastomères est de 25 minutes.

On peut facilement constater lit mime chose en comparant la figg. 11 avec la firg. 17. Les denx blastomères $i, l$ des quadrants I', I ne sont séparés que par me senle phase intermédiaire $h$ : mais dans la fig. 11, le blastomère $i$ du quadrant I' étant né à 8 h. 55 , est plus jeune que le blastomère $l$ du quadrant $A$, lequel est né ì 8 h. 20'. Au contraire, dans la fig. 17, le blastomère $i$ du quadrant $P$, né à $5 \mathrm{~h}$. 40', est plus agé que le blastomère $l$ né à 6 h. 15 .

Or, si nous comparons la différence entre les inges de ces deux hastomères $i, l$, nous verrons qu'elle est de 35 minnte's, c'estid-dire de lo minutes plus grande que dans le cas précionlent.

Enfin, si l'on compare la fig. 13 avec la fig. 18, on constatera que la diffẻrence entre les ìges des blastomères les plus arancés dans les deux quadrants $A, P$ est de 45 minutes, et, far suite, de 10 minutes plus grande que dans ies cas qui précélent. D'où l'on pent conclure qu'à chaque segmentation de ces deux blastomères, la liflérence entre leurs agres s'accroit de 10 minutes.

Par conséquent, comme, dans l'astrnchronisme accéléré, les piriorles assimilatrices des phases plus arancées deviennent successirement plus courtes; comme, l'autre part, le blastonere le plus arance du quadrant $I$ ', devient, a chaque sermentation, le 10 minutes filus jeme que le blastomère le plus arancé du quadrant $A$, il arrivera bientot un moment ou la différence entre les ages de ces deux blastomeres sera plus grande que la durée de la période assimilatrice du blastomère le plus arancé du quadrant $\mathcal{A}$. A partir de cet instant, la distance putre ces deux blastomeres ne sera plus représentée par une seule phase intermédiaire, mais par deux et, plus tard, par plusieurs phases. On peut done conclure que dians lastuchronisme arcéléé, la distance entre le blastomère le plus avancé du quadrant 1 et le blastomère le plus avancé du quarleant $I^{\prime}$ siccroit au fur et a mesure que la segmentation progresse 
et, comme conséquence, que, à parité des autres conditions, elle est d'autant plus grande que les cellules de l'agrégat cellulaire sont plus nombreuses.

Mais-dans l'asynchronisme rialenti, les choses se passent bien diflëremment. En eflet, il est vrai que le blastomère le plus arancé du quadrant $P$ est toujours plus àgé que le blastomère le plus avancé du quadrant 4 ; il est varai aussi que

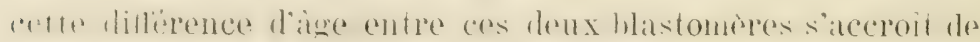
10) minutes a chacune de leur's divisions; mais, comme dans l'asynchronisme ralenti, la période assimilatrice d'une phase est de 5 minutes plus longue que celle de la phase précédente, comme, d'autre part, à chaque division, un blastomère abandome une phase pour entrer dans deux autres phases successires, dont la plus arancée a une période assimilatrice de 10 minutes plus longue que celle de la phase abandonnée, il s'ensuit que ce ralentissement de 10 minutes compense l'accroissement de l'àge; et, par conséquent, la distance entre les denx blastomères les plus arancés des quadrants $1, P$, sera trujoul's la même, c'est-i-dire que ces deux blastomères ne se:ont jamais séparés que d'une seule phase intermérliaire, quolle que soit la phase de l'érolution que l'on reuille considirer. C'est d'ailleur's ce qu'on peut constater facilement en examinant les fig. 14-19, et c'est ce que le lecteur pourra par lui-nême rérifier dans tous les cas d'asynchronisme ralenti qu'il lui plaira de supposer.

Tous pourons déduire des conclusions analogrues si nous comparons chacun des quadrants $G, D$ avec les quadrants $A, P$.

Dans la fig. 5 , le blastomère le plus avancé / du quadrant $D$ so trouve dans la phase qui précède immédiatement la phase g du quadrant 4 mais il est de 5 minutes moins âgé que celui-ci. Inans la fig. 9, le blastomère $h$ du quadrant $D$ représente une whase immédiatement précédente à la phase $i$ du quadrant A; matis il est de 10 minutes moins ìgé, c'est-à-dire que la dif-

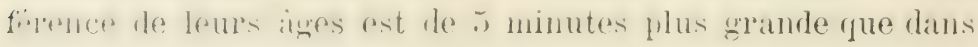
la fig. J. De même, le blastomère $k$ du quadrant $D$ de la fig. 11 
est de 15 minutes moins âgé que le blastomère $l$ du quadrant .1. On roit done qu’i chatue division de ces hlastonères, il y a un accroissement de 5 minutes dans la différence de leurs ìges. Or, tant que cette différence est inférieure à la longueur de lat périole assinilatrice du hastmiere le flus arancé du quadrant $A$, les deux blastomères les plus arancés des deux quadrants $D, A$ représenteront toujours deux des phases successives de l'érolution de l'veuf; mais des que cette diflërence sera plus grambe que la périonde assimilatrice du blatstomère le plus avancé du quatrant .1, les phases des blatstomères les plus arancés de ces deux quadrants ne seront

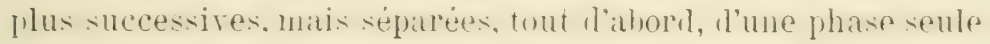
et puis de deux ou de plusieurs phases intermédiaires.

En d'autres termes, le résultat sera analogue à celui que nous arons démontré pour les quadrants $A, P$; mais, comme l'accroissement dans la différence des àges des deux blastomères des quadrants $D, A$, n'est pas de 10 , mais de 5 minutes seulement, éridemment il ne sera atteint que plus tard.

On peut arrirer à une conclusion analogue en comparant les blastomères les plus arancés des quadrants $P, D$. Le blastomere le plus atrancé du quadrant $I$ ' est toujours moins itgé que le blastomère le plus arancé du quadrant $D$, et la différence de leur's ages s'accroit de 5 minutes a chaque segmentation. Tant que cette différence sera plus petite que la périote assinilatrice du blastomere le plus arancé du qualrant I), ces deux blastonires reprisenteront deux phases successives de l'évolution; mais dès que cette différence sera plus gramle que cette périole, les phatses des loux blastomires les plus arancés seront séparées par une, deux ou plusieurs phases intermédiaires.

On peut donc conclure que, même à ce point de vue, la polarité de l'agrégat cellulaire lans l'astnchronisme accébér val saccentuant le plus en plus, at mesure que la sermentation progresse et, par conséquent, à mesure que les cellules deriennent plus nombreuses. 
Mais si, dans les fig. 11-19 de l'asynchronisme ralenti, on fait des comparaisons analogues it celles que nous renons de faire pour l'asynchronisme accéléré, on rerra fitcilement que les conséquences ne seront pas les mêmes.

En effet, le blastomère le plus avancé $f$ du quadrant $D$ (figr. 15) représente une phase immédiatement précédente a la phase du hastomère le plus irrancé g/ du quadrant .1; mais il est de J minutes plus ìgé que celui-ci. Or, cette différence dans leurs àges s'accroit de 5 minutes a chaque division de ces blastomeres; mais, pour les raisons que j’ai déjà exposées, ces deux blastomères représenteront toujours deux phases successires de l'érolution de l'oeuf, quel que soit le nombre des cellules constituant l'agrégat cellulaire à un instant quelconque de sa formation.

Lnfin, apres cette comparaison entre les constitutions de différents quadrants, il nous reste encore it examiner la constitution de chaque qualrant isolément. Mais cette comparaison n'est pas diflicile, et les conclusions se dérluisent tris aisément.

Il suffit, en effet, de considérer que chaque quadrant présente une constitution parfaitement analogue a celle de l'anréent cellulaire entier, c'est-it-rlire qu'en chaque quadrant, il y a un blastomère plus avancé, un autre moins avancé et d'autres blastonères représentant des phases intermédiaires, et que chaque quadrant, à son tour, pent être divisé en 4 parties et celles-ci, à leur tour, en 4 parties, et celles-ci encore en 4 parties etc. Evidemment, la division ultérieure possible de ces parties en 1 antres dépendra du nombre des cellules constituant l'agrégat.

par conséquent, chaule qualrant présente me polarité et une symétrie bilatérale déterminées par les mèmes conditions qui caratérisent la polarité et la symétrie bilatérale de tout l'agregat cellulaire, et chanue quatrieme de chaque quadrant grésente, lui ausi, une polinité ot une symétrie bilatérale analogues.

Or, dans chacun de ces quatrièmes de $1^{e}$, de $2^{\circ}$, de $3^{\circ}$, de 
ne ordre des quadrants, il y aura aussi un blastomere plus arancé que les autres dans l'évolution; et si nous comparons le blastomère le plus avancé du quatrième de quadrant contenant les blastomères les plus arancés arec le blastomère le plus arancé du quatrième contenant les blastomères les moins avancés (c'est-i-dire des quatrièmes du quadrant correspondant respectivement aux quadrants A, $P$ de tout l'agrégat cellulaire) on constatera que, dans l'asynchronisme accéléré et dans l'asynchronisme ralenti, existent parfaitement les mèmes relations d'àge et de phase d'érolution que nous arons constatées dans l'agrégat cellulaire entier, et, par conséquent, on pourra conclure que: dans l'asynchronisme accéléré, les blastomeres les plus avancés s'éloignent de plus en plus des autres, et que, dans l'asynchronisme ralenti, la distance séparant les blastomères les plus arancés des différents quatrièmes des quadrants se mantient toujours constante.

Le lecteur nourra comprendre la nécessité de ces comparaisons et toute la portée de ces conclusions dans les chapitres suivants.

Malheureusement, les résultats expérimentaux pouvant confirmer ces dérluctions théoriques que nous arons faites sur le rythme de segmentation ne sont pas très nombreux, parce que les Biologistes, ne pouvant comprendre toute l'importance de l'asynchronisme de segmentation, n'ont pas tenu un compte parfiatement exact du temps de l'apparition des sillons. Encore, fiut-il ajouter les difficultés de ces observations, difficultés qui vont naturellement s'accroissant a mesure que la segmentation progresse. Mais bien que les recherches faites à ce point de vue soient rares, je dois néanmoins mentionner ici deux travaux d'une grande importance pour la confirmation de nos déductions (1).

(1) Jorday E. O. and Eyclestixuer A. C. - On the Cleavage of Am. phibian Ova, in: Journ. of Morphology, Boston, Vol. IX, 1894, p, 407Ercleshymer A. C. - The early Development of Amblystoma, with observations on some other Vertebrates, ibidem, Vol. X, 1895, p. 343. 
Il sougit de l'étude de la segmentation des oenfis de quelques animaux, ou les instants de l'apparition des sillons sont enregistrés le plus soigneusement possible, et oi les auteurs arrivent à la constatation de faits très intéressants, sans qu'ils sachent en donner une explication.

Or, non seulement ces faits comcident presque parfaitement arec nos résultats théoriques, mais ils reçoivent de notre interprétation une explication naturelle et scientifique.

On constate avant tout que le laps de temps qui s'écoule entre la féconration et l'apparition du premier sillon de seagmentation n'est pas le mème pour les différents oeufs. Ce qui est naturel, d'après ce que nous venons de dire dans ce chapitre, ru que ce laps de temps dépend de la constitution bioplasmattique de l'oeuf et de la durée de la première période assimilatrice, lesquelles peurent itre différentes dians les divers oeufs.

On constate en outre que les divisions ne sont pas parfaitement synchroniques, et que l'asynchronisme est indépendant de la présence du deutoplasma plus ou moins abondant contenu dans les oeufs ou dans les blastomères. Aussi, les auteurs sont-ils annenés a conclure que la rapidité de la segmentation est dépendante d'une tendance innée et héréditaire du cytoplasme et du noyau. Mais nous venons de voir que cet asynchronisme, quoique indépendant de la quantité du deutoplasma, est tout simplement une conséquence naturelle et nécessaire de la cause même de la cytodiérèse, c'est-á-dire de l'assimilation, et le l'hétérogénéité de l'agrógat de segmentation, it la suite de son développement monodique.

Enfin on constate encore que cet asynchronisme, quoique feu ivilent an commencenent de la segmentation, devient filus manifeste, at mesure que celle-ci progresse. ce que nous venuns de démontrer of dexpliquer tres clairement par notre interprétation.

Certes, ii ne faut pas croire qu'entre les faits réels et les axemples therrigues il doit exister toujour's une coincidence fartitement exacte. Les exemples ne peuvent servir, dans ces 
cas, qu'i concréter nos idées at a laire nienx ressortir les conséquences des principes de l"interpétation. On doit donc les accepter et les juger cum grano salis, en tenant compte, d'une part, de lirrégularitis des phénonènes réels et, d'autre gart, le l'inexactitule des lonnese de l'obervation, diuns cette sorte d'expériences biologiques.

En résumé, nous pourons conclure:

$1^{\circ}$ Dans les cellules dérivées de la segmentation d'un oeuf,

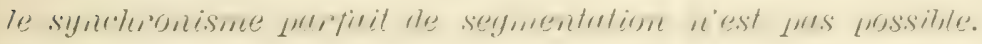
Même lorsque les segmentations nous paraissent synchroniques, en réalité elles ne le sont pas parfaitement.

$2^{\circ}$ Dans les divisions des cellules dérivées de la segmentation d'un oeuf, il y a donc toujour's un asynchronisme plus ou moins accentué. La cause de cet asynchronisme de seg-

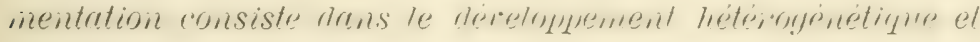

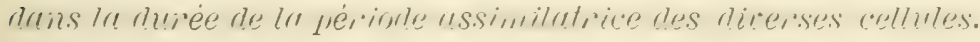

$3^{\circ}$ La valeur de cet asynchronisme produit le rythone de la segmentation.

$4^{\circ} L^{\prime}$ asynchronisme peut être ou accéléré ou bien ralenti: il n'y a pas d'autres modes possibles.

$5^{\circ}$ Aussi bien dans l'asynchronisme accéléré que dans

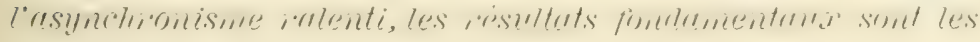
niêmes: l’agrégat cellulaire acquiert une polarité et une syinétrie bilatérale; car ces résultats sont la conséquence du léveloppenent monodique.

$6^{\circ}$ La syméti-ie bitatérale est moins la conséquence de la

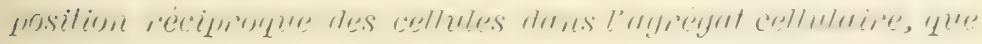
de la piésence, dans cet agiégat, des cellules homonymes ininlementrines.

7o Si l'asynchionisne est accéléré, la segmentation générale s'accélère aussi de plus en plus, la polarité de l'agrégat devient de plus en plus accentriée, et l’oeuf atteint plus tôt la

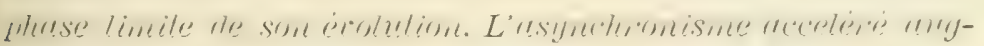
mente donc les effets du développeinent inonodique. 
So Si l'asynchronisme est ralenti, l'agrégat cellulaire présente toujour's une symétirie trilatérale; mais les effets du developrement monodique subissent en quelque mesure une attènution. La segrizentation générale se ralentil, lu polarité de l'agrégat est moins acrentuée, et l'oeuf alleint plus taid la phase limite de son évolution. 


\section{Chapitre VIII.}

\section{La première phase de l'ontogénése.}

SOMMAIRE: La spécificité orulaire - La limite dও la potentialité évolutivo de l'oeuf - La première lignée de cellules - L'entrecroisement des plans de segmentation et son importance dans la formation de L'agrégat cellulaire La production de substance de sécrétion de la part des blastoméres - La parenchymula - La morula - La blastula - Les conditions chimiques, physiques et mécaniques agissant sur la formation de la blastula - Action des substances accumulées dans la cavité de la blastula sur la direction des plans de segmentation - Importance secondaire de la forme de l'agrégat cellulaire, et importance capitalo de sa constitution - Résumé.

Loeul a donc effectur sa segmentation et, par un des blastomeres de lanrérat cellulaire qui en résulte, il est arrivé at la phase limite de sa potentialité érolutive.

Evidemment, nous ne pourons connaitre directement et exprimentalement cette phase limite; mais si lon considere qu'elle est intimement liée à la potentialité érolutive de l'oeut' et que celle-ci, comme je l'ai Témontré. est dépendante de la constitution du binplasma et de la nature chinique des sub-

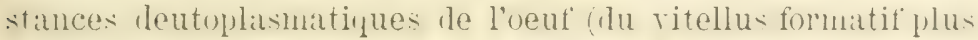
purticulierenent) on comprend facilement que, si les constitutions chiniques du bioplatinat de l'oeut, on de ses sulstances deutoplismatiques, ou de touter les deux at la tois sont dillitrentes, la phase limite sera, elle aussi, différente.

Il s'agit donc de saroir si les bioplasmats et les substances leutoplasmatiques des oeufs les dirers animaux sont égaux ou bien différents, cee que nums -ommes dans l'imposibilité de dimontrer expérinentalenent, ru l'insullisance de nos nuyen. atuels dinvestigation scientifique. Mais il sutlit de consirlérer, nème superticiellement, les phéllonènes ontogénétiques, pour 
se convaincre que cette différen ce entre les constitutions des oeufs des animaux, que cette spécificité ovulaire, comme nous pourons l'appeler, est une conclusion a laquelle on est forcément amené sinon parr les résultats des analyses chimiques, du moins par la logique des déductions et par une foule de preures indirectes. Comment pourrait-on expliquer, sans armettre cette spécificité ovulaire, que, de deux oeufs placés dins des conditions identiques du milieu extérieur, puissent dérirer deux animaux très différents entre eux? Je ne crois honc pas nécessaire de m'arrèter sur cette question, d'autant plus que je suis conritincu que les biologistes actuels ne mettront pas en doute cette spécificité, qui, d’ailleur's, s’imposo de plus en plus à notre esprit, à mesure que nos connaissances et nos expériences sur les phénomènes ontogénétiques doviennent plus nombreuses et plus rigoureuses.

Or, comme la potentialité érolutive de l'oeuf est léterminée par la constitution du bioplasma et les substances deutoplasmatiques, comme, d'autre part, ce qui caractérise la potentialité évolutive, ce sont les différentes phases do l'érolution et la phase limite, il s'ensuirra que chaque espèce d'oeuf possedera une potentialité érolutive il elle propre, et, par conséquent, son évolution sera caractérisée par une phise limite déterminée et par un certain nombre de phases intermédiaires.

Nais, conme, at parité des autres conditions, le nombre des cellules de segmentation, it un moment domné, est dépendant de la phase de l'évolution à laquelle l'oeuf est arrivé, et de la durée des périodes assimilatrices des diflérentes phases, ainsi rque je l'ai démontré dans le chappitre précédent, il s'ensuirra nécessairement que, lorsqu'un des blastomères de l'agrégat cellulitire atteindra la phase limite, à ce moment, l'agrengat résultera constitué d'un certain nombre de cellules, nombre, qui, je le répìte, sera dépendant du nombre des phases intermédiaires et du rythme do segmentation.

Par conséquent, dans charque espece d'oeuf, le nombre des 
cellules de l'agrégrat cellulaire résultant de sa segmentation à l'instant où la phase limite ra être atteinte, sera déterminé et constant. Mais quel que soit ce nombre, la constitution typique de l'agrégat cellulaire, comme nous renons de l'étitblir dans le chapitre précédent, sera toujours la même.

En disant que l'oeuf possèle une phase limite de son érolution, j'entends implicitement qu'il ne peut pas dipasser cette phase, si des conditions nouvelles n'interviennent pas pour modifier les conditions préexistantes. La potentialité érolutive do l'oeuf so réduit done, en dernière analỵse, à un phénomène très simple, c'ost-it-dire à donner origine, par des périodes d'assimilation et de dirision alternées, it un certain nombre de cellules, lesquelles, suirant le développement monorlique, représentent les diverses phases caractéristiques de l'érolution de l'oeuf. Nous avons vu, au chapitre V, comment cette potentialité pout être oxpliquée très facilement sans recourir à des forces spéciales hypothétiques; nous avons aussi vu qu'elle est une conséquence nécessaire et inéritable de la constitution de l'oeuf. Mais je crois bon d'insister sur la limitation de cette potentialité, afin de ne pas attribuer à l'oeuf une potentialité supérieure ì celle que réellement et rationnellernent il peut posséder.

L'oeuf, à lui seul, par son bioplasma et ses substances deutoplasmatiques (ritellus formatif) n'a donc que le pouroir de donner lieu it une seule lignée de cellules, que nous pourons appeler la première lignée; et si les phénomènes ne s'arrêtent pas it cette première phase de l'ontogénèse, c'est que d'autres conditions, que nous allons examiner sous peu, sont intervenues, modifiant le milieu dans lequel vivent les cellules de latgrégat cellulaire et rendant possible une érolution ultérieure.

Il est donc bien entendu que, lorsqu'une cellule de la prémière lignée arrive il la phase limite de la potentialité de l'oeuf, elle ne peut plus se diriser ulterieurement si des conditions spéciales n'interviennent pas; mais il est aussi très érident que, si les substances deutoplasmatiques ne sunt pas 
épuisées dans les autres cellules se trourant encore dans les phases intermédialires, nous n'arons pas de riasons plausibles four refuser it ces cellules la faculti de se diviser ultérieurement.

Aussi, fint-il admettre nécessairement que ces cellules des phases intermédiares continuent it se diriser et que, en suivant le déreloppement monodique, alles progressent dans leur érolution, atteignant, à leur tour, la phase limite de l'érolution de l'oeuf.

Examinons maintenant les conséquences qui dérivent de la formation de cette première limnée de cellules, c'est-it-dire les conséquences directes de la segmentation de l'oeuf.

Je crois aroir démontré, au chapitre IX de la $\mathrm{l}^{\mathrm{e}}$ partie de cet ourrage, que la direction des plans de segmentation des veufs est léterminée exclusivement par des conditions mécaniques, auxquelles les oeut's mènes ou les cellules qui dérivent de leur segrmentation sont sommis pendint la cýtodiérèse, en comprenant, bien entendu, dans ces conditions mécaniques, celles mèmes qui dépentent de la constitution ou de la torme de l'oeuf et de la présence ou de l'absence de membrane brute. Je ne reviendrai done pas sur ces problèmes; mais je ferai remarquer que, quelles que soient les directions des différents plans le segmentation, elles seront nécessairement ou parallèles on inclinées entre elles. Matis, comme les denx cellules issues de lat fremière division, ou bien sont contenues dins une menhrane, ou bien sont allerentes entre elles, il s'ensuit ividemment que, soit a callse le la pressimm, soit a callse de laulhésion, le deuxieme plan he semmentation est toujours perpendiculaire ou presque perpendiculaire an prenier. Par conséquent, les deux preniers plans de segmentation s'entrecroisent sous un angle dont la valeur pourl'a ètre trés diflérente suirant les cas que l'on comsilère; ralen' qui, d'ailleur's, n'a pas la moindre importance dans nos considérations.

Ce ru'il importe senlenient de remarquer ici, cest ques, si les plans de dirision étaient tous parallèles entre enx, les cellules 
de l'agrégat cellulaire qui en résulte formeraient une seule file, tandis que, les plans de livision śentrecroisant, l'agrégat cellulaire prend nécessitrenent me forne solihle, comme dans les oeufs hobblastiques, on. llu muins, une fimme limellate, comme dans les venfo méroblat tiques it sezmentation discondale.

En tout cas, lentrecroisement phus un moins régulier des plans de segnentation létemune, dinns latrregat cellulaire, la possibilité de la furmation a"une carité. Mair ce n'est pas lá la seule conséquence de la segmentation.

Nous sarons en effet que la dirision de la cellule et, par suite, la segmentation de loenf ne se fait liats sans assinilation préalable. Tous sarons aussi, et je l'ai démuntré dans la l" partie de cet ourrage, que l'assinilation est toujours accompagrnée de la sécrétion, c'est-it-lire que tonte biolonnlécule, en assimilant pour arrirer is soll déduublement, produit aussi des substances spéciales, que mus pourons considérer comme des produits de sécrétion et qui sont, nous l'arons ru, une conséquence directe du phénonène mème de l'assinilation. Il est donc érilent que toutes les cellules issues de la segmentation de l'oeuf, arant de se diviser, doirent assiniler et, par le fait même de l'assinilation, elles doirent produire des substances de sécrétion.

ur, ces substances penrent itre ou soliles, on liquides, ou grazeuses, et, pall suite, elles peurent diffuser ou non à l'extérieur de la cellule, suirant leur itat pligsique et suivant la nature de la membrane cellulaire, d'après les luis de l'osnuse.

C"est précisément de la production et de la rlifusibilité plus ou moins grande le ces substances sécrétées en relation avec la structure de l'oeuf et arec les conditions nécaniques réglant la segmentation, que dérivent les difrérentes constitutions morphologiques de l'agrérat cellulaire caractérisant les premières phases du déreloppement ontogénétique.

Le lecteur connremina firfaitenent que, ru le cirractère général de ce trarail, je ne pui prentre en consileration tous les cas si nombreux et si variés que la nature nous présente. 
Je suis donc forcé d'en examiner seulement quelques-uns en me proposant pour but, moins l'explication particulière de cess cals que l'indication des conditions, parfois apparemment négligeables, dont il funt tenir un compte exact pour comprendre lorigine de formations qui peurent nous paraitre dues a des forces spéciales biologiques, tandis qu'elles ne sont que des conséquences tout a fit naturelles de conditions physiques ou mécaniques.

Je supposerai donc, arant tout, que les substances sécrétées par les cellules de segmentation ne diflusent pas a l'extérieur de celles-ci.

Eridemment, dans ce cas, il ne se formera pas une cavité de segmentation ì l'intérieur de l'agrégat cellulaire, et celui-ci se présentera donc comme un amas de cellules, qui sera toujours plein, c'est-it-dire sans carité, mais dont la constitution pourra néanmoins ètre différente. Cela dépendra de la constitution de l'oeuf, et des phases de segmentation.

En effet, si l'on suppose que l'oeuf soit alécithe et dépourvu le membrane brute, le quatrième plan de segmentation sera tangentiel à la surface de l'agrégat cellulaire, ainsi que je l'ai démontré au chapitre IX de la $1^{0}$ partie de ce travail (1). Parr conséquent, a partir de ce moment, l'agrégat cellulaire présentera une couche superficielle de cellules et un amas d'autres cellules a l'intérieur de celles-ci. En peu de mots, on obtiendra ce qu'on appelle une parenchymula.

Si, au contraire, l'oeuf est pourvu de membrane brute accolée à lat surface de l'oeuf, le te plan de segmentation ne sera pals tangentiel mais radial, comme je l'ai démontré au même chapitre IX de la $1^{\circ}$ partie (2); et puisque les autres plans qui suivront anrès le quatriène auront une direction radiale toujours a cause de la membrane brute, il s'ensuivra naturellement que l'agrégat cellulaire ne sera constitué que d'une

(1) Grglio-Tos E. - Les I'roblemes de la Vie. 1e Partio, p. 255-256.

(2) Loo. cit., p. 258. 
seule couche périphérirque de cellules ot, par conséquent, on arrivera it la formation d'une momule.

Mais ce n'est pas seulement de la membrane qu'il faut tenir compte dans l'étule de ces premieres formations morphologiques de l'ontogénese. La grandeur relative des blastomères dans la segmentation inégale, et lasynchronisme de division de ces blastomères, jouent aussi un rôle de quelque importance. C'est ainsi, par exemple, que si certains blastomères sont plus petits et se divisent plus tit que les atutres, comme. d'une part, leur nombre s'accroit plus rapidement et que d'autre part ils adherent aux autres blastomères plus grands, ils tendront à couvrir ces derniers en simulant une gastrulation épibolique, ainsi qu'on le voit dans la segmentation des oeufs des Cténophores.

Ces exemples, je le répète, n'ont pour but que de rappeler l'attention des bioloristess sur less causes mécaniques pourant. provoquer des formations morphologiques diflërentes; causes que, malheureusement, nous ne pourons comnatre toujours parfititement, ce qui peut nous amener it des conclusions erronées sur l'interprétation des phénomènes ontogénétiques et sur la signification morphologique des premières phases du développement.

Ia supposition que je riens de faire, que les substances récrétées par les blastomeres ne soient pas diffusibles, bien qu'elle soit possible, est néammoins très rare. L'étude de la segmentation de la plupart des neuf's que nous connaissons nous revele au contraire que, plus ou moins tard, un liquide de sécrétion s'accumule à l'intérieur de lagringat cellulaire, et que ce liquide détermine la lormation l'une cavité qu'il remplit, c'est-à-dire d'une carité de segmentation (1). Je dirai

(1) On ne pent pas douter, même an point do rne théorique, que ces substauces s'accumulaut ì l'iutérienr de l'argrégat de segmentation ne soient produites par les cellıles mêmes de l'agrégat. Ce pluénomène a été constaté directement par LokB (Arch. $f$. Entivichelungsmech., 1895, vol. I, p. 461) dans les blastomeres de Arbacia. 
meme que lit probuction de substances diffusibles de la part Ho blastomeres ne fait jamais difaut, duoidu'elle puisse itre tris tamdire. Nons en arons un exemple dians la semmentation less outs des Mammitères, oir la formation d'un liquide à l'intérieur le l'agrégat cellulate n’a lieu qu'it segmentation très avancée.

En tout cas, l'accumulation d'un liquide à l'intérieur de l'anregat cellulaire determinera lia formation d'une blastule de corte prenière phase de l'ontogénèse, si commune et si caractéristique dans le déreloppement le la plupart des organismes animaux, et dont l'origine, ainsi qu'on le roit, ne réside que dans des phénomènes exclusirement mécaniques, chimiques et physiques, a saroir l'entrecroisement des plans de segrmentation, la proluction de substances de sécrétion, et la diffusibilité de ces substances à l'extérieur des cellules qui les ont sécrétées.

Or ces substances, dès qu’elles apparaissent dans la cavité de la blastula, jouent un ròle mécanique qui n'est pas sans importance dans la segnentation ultérieure des blastomères: rile dont on ue doit pas cependant exagérer la portée, et que nous pourrons apprécier dans sa juste valeur en analysant attentirement les combitions yui accompagnent la formation re ces substances.

Il fiut, arant tout, remarquer que l'oeuf, du commencenent de sa segmentation juscu'it la gastrulation, jouit d'une indéfendance presque absolue du milieu extériems. Je reux dire que, si l'on excepte l'oxygène, qui est, en partie du moins, fuisé í l'extérieur, toutes les autres substances chimiques servant à la nutrition du bioplasma de l'oeuf et des blastomères sont fournies par le deutoplasma, par le vitellus formatif particulierement.

Par là, je ne prétends pas nier que le milieu extérieur puice influencer de quelque manière le déraloplement de lomuf. J'mlmets parfaitement que les substances chimiques externes, et surtout les substances salines disioutes dans l'eau 
puissent exercer une action quelconnue sur les phènomenes de l'assimilation, mais je crois que ces actions sont moins l'ordre chinique que doritre phrsique. Je comprends, par exemple, que certains composés puisent, pal leur frésence, empècher ou ralentir les réactions carractéristiques le l'atssinilation on bien les troubler: nats tons ces phenomenes peurent itre expliqués par de simples actions physiques on physico-chimiques. En tout cas, il ne fiut janais oublier que, s'il est vrai que, par des expériences conrenables et par des substances spéciales dissoutes dans l'eatu dans lauplelle l'veuf est plongé, nous pourons influencer le léveloppenent de cet oeuf, cela ne proure pas encore que, dans les conditions naturelles, les substances qui se trouvent nomalenent dans le milieu extérieur puissent toujour's exercer une action quelconque physique ou chimique sur le déreloprenent de l'oeuf. En diautres termes, les actions que certaines substances exercent sur les phénomènes de l'assinilation dépendent de la nature spéciale de ces substances et de la constitution du bioplasma que l'on considere: par consirguent, elles n'ont qu'une valeur relative et on ne peut pas les généraliser.

Si donc, dans les conditions normales, l'oeuf et les blastomères lérivant le sa segrmentation ne puisent pas à l'extérieur, mais exclusirement dans le deutoplasma, leurs substances le nutrition, il sensuit érilemment que le rolume le l'algrégit cellulaire, à une phase quelconque de la segmentation, sera toujours égal au volume qu'arait l'oeuf arant le commencement de la segmentation. ("est ce que d'ailleurs on peut constater dans le déreloppenent des oeufs, du moins dans la phase de la blastulation.

Or, comme, at chaque période d"assimilation suivie de la cytodiérèse, les biomores se dérloublent et que par conséquent, la masse totale du biophasma derient double de ce qu'elle était aupararant, comme cet accroissenent de la masse hioplasmatique, conséquence directe de lassinilation, se fait aux dépens du deutoplasma, il s'ensuit éridemment que la masse 
dentoplasmatique doit d'antant diminner, que la masse biopatsmatique alugmente. P'all conséquent, durant la segmentation, il y aura un accroissement continuel du bioplasma et une diminution correspondante du deutoplasma.

Mais l'assimilation est accompagnée de la sécrétion et les substances sécrétées peuvent être consilérés comme dérivant des hiomolécules et, par suite, du hioplasmat. rependant si l'on considere que le bioplasma, à chapue périole d'assimilation, s'accroît aux dépens du deutoplasma, il faut conclure que les substances de sécrétion, quoique dérirant directement du bioplasma, sont néanmoins, en dernière analyse, des produits indirects du deutoplasma. Il est vrai, en effet, que ces substances sont sécrétées par les biomolécules; mais il est aussi incontestable que les matériaux pour leur fabrication, que les atomes, en somme, dont elles se composent, sont puisés par les hiomolécules dans le deutoplasma méme. Par conséquent, la formation et l'accroissement des substances sécrétées doivent itre accompannes d'une diminution correspondante de la masse deutoplasmatique.

Or, si les substances sécrétées, ainsi que nous le supposons dans ce cas, diffusent à l'extérieur des blastomères et s'accumulent à l'intérieur de l'agrégat cellulaire, le volume de la bistula qu'on obtiendra sera égal á celui de l'oenf arant la segrmentation; mais comme une partie du volume total de la blastula est occupé par le liquide que celle-ci contient. il s'ensuivra que le volume de chaque blastomère subira une diminution correspondant an rolume des substances qui diffusent, et que, par conséquent, l’accroissement du liquide ì l'intérieur de la blastula sera toujours accompagné d'une diminution équiralente du volume total des blastomères.

Toutes ces considérations sont indispensables pour comfrendre exactement l'action mécanique que le liquide de la blastula peut exercer sur la division des blastomères.

Tant que les blastomeres ne puisent pas leur nourriture dans le milieu extérieur, le rolume de l'agrégat ne peut aug- 
menter, et par suite, vu la correspondance que je viens de démontrer entre la diminution du rolmme dos blatstomères et le volume du liquide de la cavité de la blastula, le rolume de ce liquide ne sera jamais plus grand que la capacité de cette cavité. Par conséquent, ce liquide ne pourra exercer sur les parois de la blastula une réritable pression hydrostatique.

Or, si cette pression hydrostatique existait et qu'elle agit à l'intérieur de la blastula, celle ci derrait prendre toujours une forme sphérique: matis comme elle llexiste pas, lat forme de la blastula ne sera pas changée par lat présence du liquide, et se conseprera toujunts ézalde it colle meme que les conditions phrsiques ou mécaniques de l'oeuf forme de l'oeuf, présence ou absence de membrane hrute, surnentation égale on inégale) auront déterminée.

Toutefois, bien que lo liquide de la carité de segmentation ne puise pas modifier lat forme de la bistulat, lat segmentation nltérieure des blastomères pourra subir des modifications.

Il faut se rappeler toujours que la formation d'une cavité à l'intérieur de la hastula est un phémomène étroitement dégendant de l'accumulation du liquide. L'entrecroisenient des flans de segrnentation est une condition nécessaire do la formation d'une cavité, mais non une condition suflisante.

Il est une condition nécessaire en tant que, si cet entrecroisement n'existait pas, la formation de la cavité ne serait pas posible, comme je l'ai démontré; il est une condition par elle soulo insuflisante, en tant que, s'il ne se formait pas de liquide. et si celui-ci ne s'accumulait pas à l'intérieur de l'agrégat, les hastomeres ne secartoraind fats l'un de lautre pour permettre cette accumulation et, par suite, la carité ne se formerait pas.

L'accumulation des substances sécrétées à l'intérieur de l'agrégat cellulaire est done la cause rraiment efliciente de la formation de la carité de la blastula et, par conséquent, on comprend facilement que si cette cavité n'est jamais plus 
petite que le volume du liquide qu'elle contient, elle ne peut itre mon plus jamais plus gramile que ce rolume. En peu de mots, la capacité de la gastrula est toujours parfatement correspondante au rolume du liquide qu'elle contient, et, par suite, cette carité sera, ¿̀ un moment quelconque de son développement, parfaitement remplie de ce liquide.

Il s'ensuirra donc que si, par exemple, le fuseau de division liun des blastomeres prend une direction radiale, comme, à un certain moment de lia cytodiérese, lat cellule est forcée de $\therefore$ allonger (I Partie, p. 177, 2le loi rationnelle) dans la direction de l'axe du fuseau, le blastomere tendra à s'allonger dans la direction du rayon de la blastula. Et si cet allongement est empèché du crité extérieur, soit à cause de la membrane brute accolée a la hastula, soit a cause de l’athésion des autres blastomeres, le blastomère en dịision tendra a s'allonger du crité intérieur. Il sera donc forcé de farre saillie dans la cavité et, par suite, tendra de léplacer une partie du liquide qu'elle contient. Or, comme celui-ci ne pent être léplacé parce qu'il remplit parfaitement la carité, il subira une pression de lat part du hastomere qui fait saillie. Mais cette pression du blastomère sur le liquide, ru l’incompressibilité de celui-ci, se transformera en une pression équiralente du liquide sur le blastomere. Alors, sous l'action de cette pression, le fuseatu, qui, nous le savons ' ${ }^{\mathrm{e}}$ Partie, p. 175, 20 loi rationnelle de la cytodiérèse, est parfitement mobile dans la cellule, se déplacera ef prendra we direction plus ou moins oblique ou même tangentielle, d'après les conditions de pression et d'athésinn léterninés par la présence et l'état physiologique des hastomeres. Io plan de division ne pourra done etre parfaitement tangentiol, matis plus on moins oblique ou meme radial.

Le liquile de la carité de segmentation peut donc exercer we pressim hylrostatique sur les parois de la blastula; mais cotte fmosion se manifestera seulement lorsque les blastomeres, en se divisant, temdmont à faire sallie daus la cavité of comprimemont eux-menes le liquide contenu dans celle-ci. 
Le lecteur comprendra parfaitement que, par cet exemple et par ces considerations, je n'ai en d'antre intention que de faire comnaitre dans sit juste valesur quelle pst l'action que le liquide de la hastula peut exercer sur la segmentation. Les conditions et les raphorts mécaniques que la mature noms présente peuvent ètre parfois bien plus compliqués, et par conséquent, lat solution des problines hien plus dillicile on mème impossible, ru la dilliculté ou l’impussibilité l'apprécip ces conditions à leur juste valeur. Mais, en tout cas, il ne faudra janais conclure que la dispusition des collules dans l'agrégat cellulaire, la forme de cet agrégat et la direction des plans

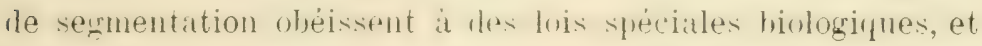
ne sont pas sommises exclusivenuent anx lois générales de la mécanique et de la physique.

Je ne m'arrêterai pas à considérer la formation de la blastula dans les oeufs it segmentation discondale. Il s'agit ici de phénomènes élémentaires lérirant de la présence d’une quantité de dentoplatina trup gramle en comparaison de la masse du hioplasnat. De lit l'impossibilité, de la part du deutoplasma, de contracter des rapports de position arec toute la masse deutoplasmatique pendant la cytodierese, et, par suite.

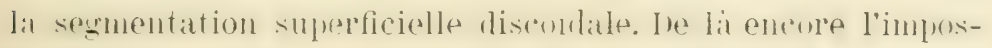
sibilité d'un entrecroisement complet des plans de semmentation, et, par consérquent, la formation d"un arrégat cellulaipo dont les cellules ne forment pas un amaś, mais, s'étalant à la surface de l'oeuf, constituent une lame cellulaire. Il s'ensuit que les parois de la blastula ne seront pas formées de tous côtés par des cellules, ainsi que nous le royons dans les oeufs a segmentation totale égale ou inégale; mais son plancher pourra être, en partie au moins, constitué par les substances mêmes deutoplasmatiques.

D'ailleurs, quelle que soit la forme de la blastula dans les différentes espreses danimatux, alle n'at, dans non interpréta-

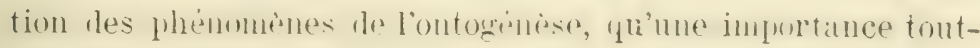
a-fait secondaire. 
La forme de la hlastula est dépendante, ainsi que nous renons de le roir, du morle d'arrangement des blastomeres qui la constituent, et d'autre part, ce mode d'arrangement est une stricte conséquence des conditions physiques ou mécaniques. Si dans une même espece d'animaxx, la forme de l'agrégat cellulaire résultant de la sermentation est constante, c'est que les oeufs de cette espece ont tous la meme constitution, et que les conditions physiques on mécaniques agrssant sur la segmentation sont toujours les mèmes. Si dans les diverses especes d'animaux, les aurégats cellulaires de la segmentation sont différents, c'est que less oeufis de ces espèces sont différents et, par conséquent, les conditions physiques on mécaniques de la sermentation peurent être, elles aussi, lifférentes.

L'agrégat cellulaire résultant de la segmentation, qu'il soit. une parenchymula, ou bien une morula, ou bien encore une blastula, n'a pats d'importance au point de vue morphologique. La forme de ce premier agrégat cellulaire ne peut influer sur la forme des oryanes qui se développeront ultérieurement, et si l'on observe que la blastula est une formation plus fréquente que toutes les autres et, par suite, plus caractéristique, la cause en est tout simplement au fait que je viens de faire remarquer, a saroir l'accumulation d'un liquide a l'intérieur de. l'agrégat.

Ce qui a la plus grande importance dans mon interprétation de l'ontogénèse, ce n'est pas la forme de l'anrégat cellulaire, mais sa constitution, c'est-i-dire la présence de blastomères telle que je viens de l'exposer au chapitre précédent; at comme cette constitution, ainsi que nous l'arons ru, est tout-i-fait indépendante des conditions physiques on mécaniques agissant sur lat segmentation, mais qu’elle dérive exclusirement du déreloppenent nonodique et de l'asynchronisme de segmentation accéléré ou ralenti, il s'ensuit évilemment que la constitution de l'arrégat cellulaire, qu'il soit une parenchymula, une morula ou bien une blastula, ne changera pas substantiellement. 
En résumant, nous pourons conclure:

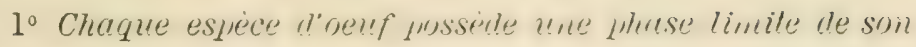
évolution déterminée par sa constitution.

$2^{\circ}$ La première phase de l'ontogénèse consiste tout sim-

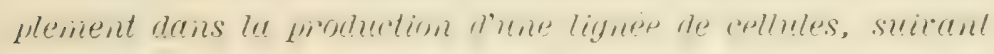
le développenent monodique. A ce phénomène se borne la polentialité érolutive de l'oenf.

$3^{\circ}$ La forme de l'agrégat cellulaire résultant de la segmentation est dependante des rmations chimiques, physiques et mécaniques dans lestruelles "lim la segmentation. Elle"

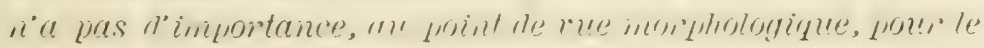
déreloppement ultèrieur de l'embryon.

$4^{\circ}$ Ce qui a la plus grande importance dans l'agrégat cellulaire de la segmentation, ce n'est pas sa forme mais sa

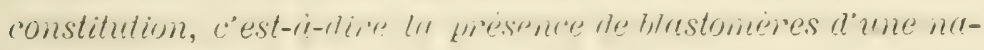
ture déterminée.

$5^{\circ}$ La formation de la blastula est une conséquence de

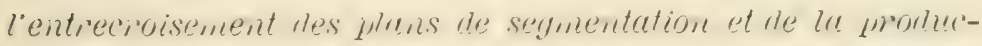

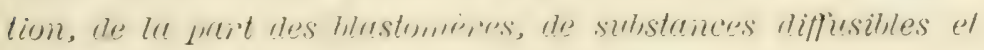
s'accumulant à l'intérieur de l'agrégat celiulaire.

$6^{\circ} L^{\prime}$ accumulation de substances ri l'intérieur de l'agrégat cellulaire peut infuer sur la direction des plans ultérieurs de segmentation. 


\section{Chapitre IX.}

\section{La deuxième phase de l'ontogénèse.}

SONMAIRE : L'oeuf et son milieu interne - Origine dece milieu - La probiose de l'ovocyte, et son importance dans la segmentation de l'oeuf' - La probiose des blastomères et la cróation du milieu interne de la blastula - Rôle de ce mileu dans la production de la deuxième phase de l'ontogénèse - La dellxième lignée de cellules - La différenciation histologique accompagnant la prolifération cellulaire - La différenciation morphologique - La gastrulation et ses causes mécaniques - La localisation des différenciations - Résumé.

Le résultat direct de la segmentation de l'oeuf est donc la formation d'un agriogat de cellules, dont une est arrivée à la phase limite de l'érolution de l'oeuf et dont les autres se trouvent à des phases diverses intermédiaires, ainsi que je riens de le démontrer. A l'intérieur de cet agrégat, sont contenues des substances spéciales, produits de sécrétion des cellules mêmes de segmentation.

Or, comme tout organisme, quels que soient sa complexité et le nombre de ses cellules, pent toujours être envisagé, au point de vue de sa constitution, comme un agrégat de cellules plus ou moins complexe, contenant à son intérieur un mélange de substances différentes, qui constituent son milieu interne, il est évident que nous pourons aussi considérer l'agrégat cellulaire résultant de la sermentation de l'oeuf' comme un ormanisme d'une tris rrinde simplicité, oi les cellules en sont les éléments constituants et les substances contenues à son intérieur en représentent le milieu interne.

Inintenant, arritons-bous quelyur peu it allalyser les causes primitives de la formation de cet organisme, et nous en tirelons des comnatissinces qui ne seront pas sans importance 
pour la compréhension des phénomènes ultérieurs de l'ontogénèse.

Quelle est la causes de la formation de ce petit organisme, la blastula, et de son milieu interne?

Nous le savons parfaitement. C'est l'oeuf. La nature des différentes phases de l'érolution de l'neuf, lat phase limito, les réactions de lassimilation, la nature des substances sécrétées et, par conséquent, la constitution du milieu interne de la blastula sont dérivées directenent de lit constitution de l'oenf. Celle-ci est donc la cause prinitive les éléments constituants, aussi bien que du milieu interne de la blastula.

Mais l'oeuf, lui aussi, peut être considéré comme un organisme unicellulaire. Il posienle un nilieu interne résultant de l'ensemble des substatices deutoplatsmatiques qu'il contient: il possède une constitution binplasmatique. Il a donc toutes les qualités nécessaires pour itre considéré comme un organisme, "quoique d'une très grande simplicité (1). Oi', quelle est l'origine de sa formation?

Noun sarons que les cellules greminales donnent naissance, par des dirisions répétées, aux orogonies; que ces orogonies, par d'autres divisions, donnent lieu it des cellules qui, après une phase d'iccroissement, deriement les orocrtes de premier ordre; qu'ia l'achèrenent de la phase d'accroissement,

(1) Afin d'éviter tout malentendu, je crois bon d'ajouter ici quelques éclaireissements sur la siguitication qu'on doit donner à l'expression de milieu interne de l'oenf. Nous savons quo l'oouf est coustitú de deux parties: le bioplasma et lo deutoplasma. Le bioplasma est l'ensemble de tous les biomores, particules vivautes de l'oenf, plongés dans le liquiclo interbiomorique. Ce liruide forme dunc le milien interne bioplasmatique de l'oeuf. Le deutoplasma au contraire est l'ensemble de toutes les particules brutes de l'oenf. Il constituo ce que j'appelle lo milieu interne de l'oeuf. Celni-ci ne doit donc pas être confondu avec le milieu bioplasmatique. Ces milienx sont tons denx à l'intérieur de l'oeuf; mais le milieu bioplasmatique est a l'intérienr mime du hioplasma, tamelis que le milieu constitué pas lo deutoplasma se trouve ì l'iutérieur de l'oeuf', mais à l'extérieur du bioplasma. 
Les orocrtes de premier ordre se trinstorment en orocytes de deuxieme ordre en émettant le premier globule polaire; et

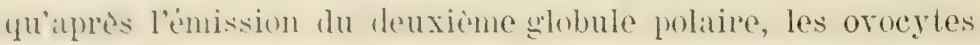
de denxieme ordere se transforment en ovules capables d'etre fëcondés, et, par sulte. de se transformer, par la lëeondation, en véritables oeufs, tels que nous les envisageons dans ces consilérations.

Les phases de cellules germinales, d'orogonies et d'orocytes représentent done autant d'étines que les cellules germinales doivent franchir pour arriver i la phase d'ovule, phase limite de leur érolution. Nous pouvons done conclure que la constitution de l'orule est la conséquence directe des phases qui précedent la formation et la maturation de l'ovule.

Nous savons encore que la phase d'accroissement, aboutissant à la formation de l'ovocyte de premier ordre, est caractérisée par l'accumulation, it l'intérieur de la cellule, d'une quantité plus ou moins grande de substances deutoplasmatiques, constituant, par leur ensemble, le deutoplasma de l'orule. Or, ces substances sont des produits que le bioplasma de l'ovocyte sécrète pendant toute la durée de la période d'accroissement. Il est vrai qu'it cette production peuvent parfois concourir indirectement d'autpes cellules, par exemple, les cellules folliculates de l'matre de certains animanx: mais, dans ces cas mêmes, on peut considérer la sécrétion de ces substances comme dépendant toujours directement du bioplasma de l'orocyte, en ce sens, que les cellules folliculaires ne produisent pas les substances deutoplasmatiques, mais, tres probablement, ne font que préparer les matérianx que le bioplasmal te l'oroçte doit transformer ultérieurement en deutoplasma.

Or, bien que l'ovocyte de premier ordre soit, au point de vue de son individuatité morpholugique, la méme cellule que l'orule; en d'autres termes, bien que nous n'apercevions pas de differences morphologiques accentués entre l'ovocy te arant

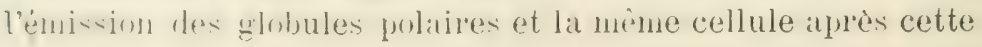


émission, il est néammoins évident qu'une différence très profonde doit exister entre ces deux phases de l'érolution d'une mème cellule. Je ne réserve de démontrer dans une autre partie de ce travail en yuni comsiste cette diférence: nous le remons quand nous etulierons les phénonènes intines de la maturation de loeuf. Mais, des a present, nous pourons nous convaincre de la réalité de cette diflérence an considérant tout simplement, que, si l'orocyte de premier ordre n'arait pas, avant l'émission des grobules poliares et, par suite, pendant la période daccroissement, une constitution bioplasmatique profondément diflérente de celle de l'orule, il derrait être, lui aussi, capable d'itre féconlé et de donner lieu au déreloppernent d'un embryon, tout comme lorule. Cee qui n'arrive pas, ainsi que nous le sarons.

Par conséquent, étant adnis que l'uroçte est différent de l'orule, comme les substances deutoplasmatiques sont produites par lorocyte, et comme ces mêmes substances servent plus tard de nourriture it loeul pendant la preniere phase du déreloppenent ontogénétique, ainsi que je l’ai démontré, il est évilent que nous sommes ici en présence de ce fait que les substances sécrétées par une cellule qui a récu arant, deviennent la nourriture liautres cellules qui virront après. En peu de mots, nous arons un premier exemple, assez frappant, de l'inportance que peut aroir dans l'ontogénèse le phénomène de la probiose, c'est-it-rlire de la vie antérieure des êtres, ainsi que je l'ai exposée dans la Ie partie de ce travail (1).

L'oeuf est done un orwanisme dont la constitution bioplasmatique est la conséquence directe des phases direrses de cellule germinale, dorognie, et d'orocrte, par lesquelles il est passé pour arriver à la phase d'orule, de même que la blastula ou l'agrégat cellulatre, quel qu'il soit, résultant de la segmentation, est un organisne dont la comstitution est la

(1) Giglio-Tos E. - Les Problemes de la Vie. $1^{\ominus}$ Partie, p. 105. 
conséquence directe des phases caractérisant la potentialité évolutive de l'oenf.

Les substances deutoplasmatiques formant le milieu interne de l'oenf' sont des prorluits que l'oeuf même sécrète en accomplissant son érolution qui l’amène ì sa structure définitive d'oeuf mûr, et les substinces fermant le milieu interne de la blastula sont des produits de sécrétion des cellules mêmes qui la constituent.

On peut done conclure que dans l'oeuf, aussi bien que dans la blastula, le milieu interne est une creation de ces organismes mêmes.

Cette création du milieu interne nous apparait d'une très grande importance, si l'on considère quelles sont les conséquences qui en découlent. En effet, si l'oeuf fécondé et dans des conditions physiques farorables peut se segmenter, suirre son érolution caractéristique et accomplir la première phase de l'ontogénèse, c'est graice i ses substances deutoplasmatiques. En sécrétant celles-ci, il a donc rendu possible son érolution ultérieure. La première phase de l'ontogénèse, la formation de la blastula, est donc l'effet direct de cette création du milieu interne de l'oeuf.

Il suthit maintenant de suirre un raisonnement analogue, pour comprendre parfaitement quelle est la cause de la deuxième phase de l'ontogénèse.

si l'orocyte de premier ordre, à sa phase d'accroissement, n'arait pas accumulé les substances deutoplasmatiques constituant le milieu interne de l'orule, celui-ci, quoique féconde, n'aurait pu se segmenter et accomplir la première phase de l'ontogénese, faute de la nourriture nécessaire pour l'assimilation de son bioplasma. De même, si, pendant la segmentation, les cellules de la blastula n’avaient pas sécrété les substances romplissant la carité de celle-ci, les blastomères, arrivés à la phase limite de l'érolution de l'oeuf, ne prourraient suive une évolution ultérieure.

La prossibilité d'un développement ultérieur de ces blasto- 
mères est dépendante de leur constitution spéciale et de la nature des substances de la carité de la blastula, tout comme la possibilité de l'évolution de l'oeul' est une conséquence de la constitution de l'ovule mûr et de ses substances deutoplasmatiques.

Pour expliquer la deuxiène phase de l'ontogénèse, nous n'arons donc qu'a supposer que les substances de la carité de la blastula puissent servir le nourriture au bioplasma des blastomères arrivés a la phase limite de l'írolution de l'oeuf. Et je crois qu’il n'y a lá rien d'impossible et rien de plus naturel.

On pourrait mobjecter que les substances de la carité de segmentation sont des proluits ile sécrétion des cellules de segmentation et que, par conséquent, il n'est pas probable qu'elles puissent servir de nourriture it ces cellules mêmes. Mais en réalité, cette ohjection n'á pas la moindre valeur.

En efret, ces substances sont produites par les cellules de segmentation, arant que celles-ci aient atteint la phase limite de l'érolution de l'oeuf, et par suite, lorsqu'elles se trourent encore dans l'une des phases intermétiaires. Mais comme, dans mon interprétation, je suppose que ces substances servent de nourriture au bioplasma du blastomere arrivé à la phase limite, par exemple au blastomère plans l'exemple que nous arons considéré, il est tout-i-fait possible et naturel que ces substances représentant des proluits de sécrétion des cellules b, (2, r, etc. puissent représenter les matériaux de nutrition pour la cellule $p$.

Ici encore, nous sommes en présence d'un phénomène parfaitement analogue á celui que nous arons considéré tout-àl'heure a l'égard de l'orule et de ses phases de préparation et de maturation.

C"est un fait incontestable que le bioplasma de l'oeut' mûr puise sil nourriture dans le deutoplasma; et pourtant, nous sarons parfatement que celui-ci est un prorluit de sécrétion de la phase d'orocyte le premier orilese par latuelle l'orule mûr est passé avant d'arriver à la maturation. 
Rien donc de plus naturel que d'armettre qu'entre les substances de la carité de segmentation et le blastomère it la phase limite se passe un phénomène tont-i-fait analngue. Il s'igrirait ici d'un autre phénomène de probiose, où la vie antirieure des cellules de segmentation préparerait le milieu interne nécessaire et firrorible i la rie des autres cellules qui virront plus tarl. En peu de mots, la première phase de l'ontogénèse serait la cause immédiate de la deuxième, en ce sens qu'elle donnerait lieu ì une cellule d'une nature déterminée, laquelle, se nourrissant aux dépens des substances mêmes sécrétées par les cellules de cette première phase, produirait la deuxième phase de l'ontogénèse.

Je supposerai donc, dans mon interprétation, que lorsqu'un blastomère arrive à la phase limite de l'érolution de l'oeuf, il acquiert une constitution bioplasmatique telle que les substances accumulées dans la carité de segmentation puissent entrer en réaction arec ses biomolécules et que, par conséquent, celles-ci puissent suivre un déreloppement hétérogénétique, tout comme nous l'arons supposé pour les biomolécules de l'oeuf.

Tous n'arons maintenant qu'i faire ici une application des principes énoncés au chapitre $V$ sur la potentialité évolutive de l'oeuf, pour comprendre fitcilement et parfaitement comment la constitution bioplasmatique du blastomere d'une part, et la nature les substances de la cavité de segmentation d'autre jart, doivent déterminer dans ce blastomère une potentialité érolutive, tout comme dans l'oeuf le bioplasma et les substances deutoplasmatiques déterminent la potentialité érolutive caractéristique de l'oeuf, ainsi que je l'ai démontré.

Cependant, conme toute potentialité évolutive est dépendante, d'une part, de la constitution du bioplasma, d'autre part, le la nature les substances nourrissantes, il est évident que la potentialité érolutive de ce blastomère ne sera pas égale à celle de l'oeuf.

En eflet, la constitution bioplasmatique du blastomère à 
la phase limite, dans notre pxemple du hastomere pl, ne peut itre égale a celle "le loueuf. Ine mine, la nature chimique des substances de la carite de sementation, que jappellerai $a_{1}$, neest pas identirue a celle des substances dentoplasmatiques ir de lonuf. La putentialité erolutire du hastomere 1 , résultant de sa constitution $p$ et de la nature $x_{1}$ des substances nourrissantes, seral donc diflérente de la putentialité érolutive de l'oeul, et nous pourrons lit representer, afin de mieux concréter nos idées, par la série des phases: $\ell^{\prime}, c^{\prime}, d^{\prime}$, $e^{\prime}, f^{\prime} \ldots m^{\prime} \ldots n^{\prime} \ldots o^{\prime} \ldots l^{\prime}$, ou la lettre $y^{\prime}$ est la phase limite de cette potentialité, et oi les autres en représentent les phases intermédiaires.

Je ne répèterai pas ici, pour plus de brièreté, les considérations exposées aux chapitres $V$ et VI pour démontrer la nécessité du dérelopennent monolique. J'r renroie le lecteur. Je me bornerili seulenent it établir que, lans ce cas, non moins que dans le précédent, l'hryuthèse dun dévelompenent polrodique nous amènerait ì des conclusions inconciliables arec les phénomènes ontogénétiques.

I'ailleur's, ce n'est pas senlement par analogie que je suppose que les substances de lil carité de segmentation foumissent la nomriture aux hastmères. Les faits mèmes qui font suite à la formation de la blastula sont une preuve érirlente qu’il s'agit d'un phénomène réel.

En eflet, si l'absorption de ces substances de la part de certains bistomères est un fiit réel, nous derons constater, après la formation de la hlastula, une diminution et mème une complete disparition srarluelle le ces suhstinces. or, cest précisément ce que nous constatons ficcilement rendant la gastrulation.

Le phénomène de l'invagination d'une portion de la fraroi de la blastula caractérisant la phase de la gastrulation, proluit érilemment et inéritahlenent. une diminution de la carité de segmentation. or, comme cette cavité est renplie de liquide, l'inragination ne serait absolument par possible sans 
une diminution de ce liquide: et si celui-ci diminue, puisque nous ne le voyons pas diffuser ì l'extérieur de la blastula, cest qu'il est absorbé par certatines cellules do la blastula mème.

Ia diminution de ce liquide peut itre plus ou moins forte: elle atteint son degré le plus éleré, lorsque la partie invaginée de la blastula, le feuillet interne, s'accole parfaitement au fenallet externe, de manitre que la cavité de segmentation devient tout-i-fait virtuelle. Evidemment, dans ce cas, le liquide de cette carite est totalement ou presque totalement dispartu:

Irais une preuve indirecte de l'ahsorption de substances de la carité de segmentation par les blastomères mèmes, nous l'arons aussi dans l'accroissement en surfice de l'agrégat cellulaire pendant la gastrulation.

Nous voyons en effet qu'à l'achèvement de la gastrulation, le feuillet interne est plus on moins accolé au feuillet externe et que le blastopore, d'abord large, se restreint peu à peu. La surface totale des cellules constituant la gastrula, c'est-d-dire la somme de la surface externe et de la surface interne de celle-ci, doit donc itre presque double on, du moins, bien plus grande que la surface qu'avait auparavant la blastula.

ret accroissement de surfice est di évidemment it l'augmentation du nombre des cellules constituant l'anrémat cellulare. Eit comme celle-ci est l'eflet de la division des cellules; comme, l'antre part, la division doit itre précédée de l'assinilation, il est évident que les cellules formant auparavant les parois de la blastula ont dì assimiler pour arriver it se diviser.

Or', si l'assimilation avait lien aux dépens des substances dentoplasmatiques, il y amrais tout simplement angmentation du nombre des cellules, sins accroissement de la surface de l'aprérat cellulate, ainsi que nous le royoms daus la formation de la blastula. Nais comme cet accroissement de surface est bien réel, il faut en conclure que les substances servant à lassinilation de cellules le la blastulat ne sont pas puisees 
dans le deutoplasma, mais on lehol's de celui-ci; et puisque, dans la plupart des cas au moins, l'agrégat cellulaire est, dans cette phase encore, indipendant du milien externe, ainsi que le prouve la constance de son volume, il est évident que la nourriture des cellules ne peut être fournie que par les substances de la cavité de segmentation.

D'ailleurs, je suis parfaitement convaincu que tout biologiste qui veut analyser attentivenent les phénomènes qui se passent dans ces phases de l'ontogenèse ne peut qu'arriver à des conclusions analogues ou concorilantes arec celles memes que je viens d'exposer. In reste, j'expère que les résultats des expriences ne tarderont pas a nous donner une preure directe de l'exactitude de mon interprétation.

A l'instant mème où le blastomère arrivé a la phase limite de l'érolution de l'oeuf, se nourrissant aux dépens' des substances de la cavité de segmentation, produites par la probiose des cellules précédentes, se divise et suit une érolution ultérieure, a donc lieu le commencement de la deuxième phase de lontogénèse. A part toute morification morphologique que ce phénomène peut entrainer, la deuxième phase ontogénétique est donc caractérisée par la simple formation d'une deuxième lignée de cellules, parfaitement comparable it la prenière lignée caractérisant la première phase.

Cependant, on comprend facilemsnt que cette denxième prolifération cellulaire doit être accompagnée d'une diflérenciation histologique.

bin effet, puisque la différenciation histologique, ainsi que je l'ai démontré an chapitre Ir, se réduit, en dernière analỹe, a me diflérenciation chimique du bioplasma des cellules, il est évident que les cellules de la nonvelle lignée, dérivant des réactions assimilatrices entre les suhstances , ${ }_{1}$ de lat cavité de sermentation et le biatomere 1 , differeront, par leur constitution bioplasmatique, les cellules de la première lignée lérivées des réactions assinilatrices entre le bioplasma a de l'oenf et ses substances deutoplasmatiques $x$. Cette différence 
de constitution bioplasmatiune poinrait hien ne pas se réréler far whe differenciation histologique saisissahle par nos moyens d'inrestigation, que cela nexclurait pas l'existence rélle de celle-ci, dans le sens que j’ai donné à ce mot au chapitre II.

Les substances de la carité de segmentation auraient done pour eflet, dans mon interpretation, de diriger les cellules de l'atgresat cellulatre, de lat hitstula, dans me roie nourelle les amenant à une autre diffẻenciation histologique.

Mais en même temps que ces phénomènes s'accomplissent, des monlifications dans la forme do l'agrégat cellulate doivent nécessatrement intervenir, à canse mème de la prolifération cellulaire et des conditions physiques et mécaniques dans lesquelles celle-ci a lieu.

On peut comprendre facilement que l'absorption des substances de la cavité de segmentation d'une part, et l'accroissement du nombre des cellules de l'agrégat de l'autre, doirent prorouper, en thise générilte, des modifications dans la forme de ce dernier. Mais dans l'éturle de ces morlifications, de meme que dans l'étude de la forme de l'agrégat cellulaire dérivant directement de lat segmentation, il faut tenir compte de toutes les conditions physiques et mécaniques pouvant influer sur ces phénomènes.

Prenons d'abord en considération un exemple simple et typinue. supposons une blastula lont les parois soient formées de cellules de la même grandeur et également adhérentes entre elles, et dont les substances de la cavité de segmentation soient liquides.

Tant que l'assimilation des blastomères se fait aux dépens des substances deutoplasmatiques contenues dans les blastomères mêmes, le volume du bioplasma de ceux-ci deviendra double à la fin de chaque période assimilatrice, mais le rolume total des biatomeres n'angmentera pas. Ils deviendront. même plus petits à mesure que la segmentation progresse, à cause des substances qu'ils sécrèteront et qui s'accumuleront dans la cavité de segmentation. 
Mais aussitot qu'un des blastomères, arrivé í la phase limite de l'évolution de l'veuf, se nourrira des substances de la carite de lat hastulat, celles-ci diminueront, tamlis que lo hlastomère, à la fin de sa période assimilatrice, aura doublé son rolume. Il exercera fonc inérithlement une presion sur les autres rellules de l'agrégat cellulatere et cette pression conttinuera quand il se divisera.

Or, si le liquide interne de la blastula n'arait pas diminué,

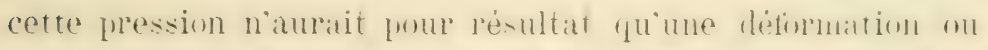
un aplatissement flus accentur dex atutes cellules de lat blatstula; mais comme le liquide interne à été en partie absorbé, et que, par conséquent, il est diminué, il s'ensuit nécessairement que la paroi de Ta blastula s'infléchira rers son intérieur. Il s'agit maintenant de déterminer le point de cette inflexion.

Or, si la paroi de la blastula est parfaitement homogène dans toutes ses parties, la détermination de ce point d'inflexion sera d'une dillioulté extratorlinaire et je lirai méme inpussilile. Fons serions, dans ce cals, en présence d'une question analogue a la détermination du print te rupture d'un fil farfaitement homogène et soumis it une tension. Mais comme l'homogénéité parfaite de la paroi de la blastula n'est pas posible, le point d’utiexinn, qui derimula le point d'invayi-

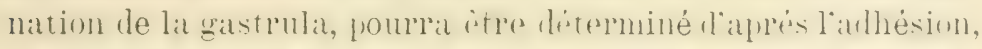
lat compresibilite, un les antres propriftés physiques dex cellules de la blastula, ou bien encore d'après les conditions

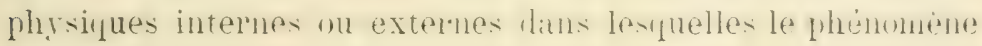
a lieu.

C'est ainsi, par exemple, que si la segmentation n'a pas été très inégale et que tous les blastomères aient à peu près le mème volume, une partie de la paroi de la blastula, comprenant un certain nombre de cellules, pourra s'infléchir et

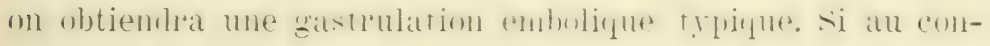
tratre la segmentation a été fotale mais tris inégale, lat partier de la bastula comprenant les blastomeres plus grands ne 
pounrar s’infléchir, à cause de la rigidité relative que cenx-ci opposoront d l'inllexion et, par conséquent, celle-ci se manifesterar al l'endroit oi cette rigidité sera plus faible. C'est ce que nous royons dans la gastrulation de la plupart des Amphibiens.

Dans le cas des oeufs à segmentation partielle discoüdale, anra lieu un phénomène analogue. Linflexion se produira évidemment a l'endroit de la résistance la plus faible à l'inflexion, et, par suite, aux limites de l'aire germinative avec l'aire ritelline. Nous en arons de nombreux exemples dans la gastrulation des Oiseaux et des Reptiles.

Lnfin, si l'invagination n'est pas mécaniquement possible, parce que la carité interne de la blastula est occupée par d'autres substances solides, ou bien encore parce que la forme de la blastula ne permet pas une inflexion typique de ses

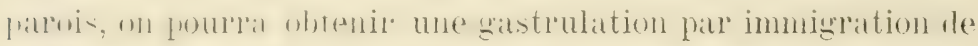

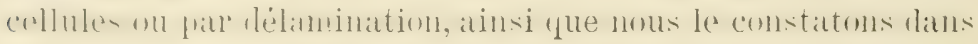
le développement de certains Coelentérés.

D'ailleur's, ici encore, tout comme pour la formation de la blastula, le lecteur comprendra facilement que je ne puis considérer tous les cas particuliers. Je ne fais au contraire

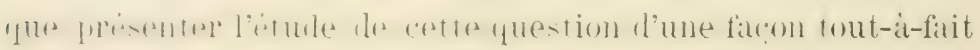

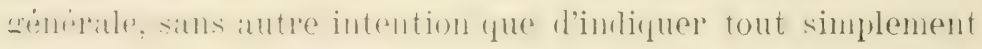
comment cette intéressante phase de l'ontogénèse peut être

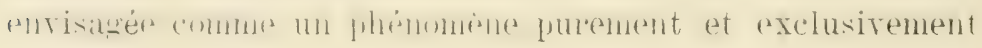
mécanique.

I)ans les difrérents cas spéciaux, le problème mécanique de la gastrulation ne pourra ètre résolu qu'en examinant et en appréciant à leur valeur exacte toutes les conditions physiques et mécaniques dans lesquelles il doit s'accomplir. Cependant, comme la connaissance et l'appréciation exacte de ces conditions sont d'une dilliculté extrème et parfois même impossibles, on sera tenté de voir dans la gastrulation un

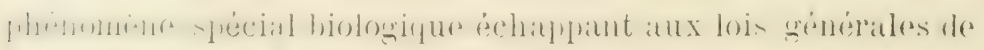

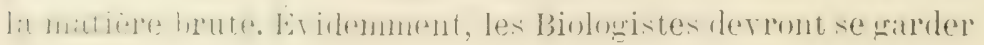




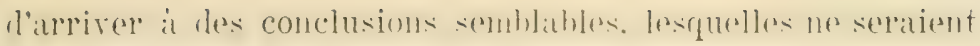
que la manifestation de la paurreté de nos commaissances.

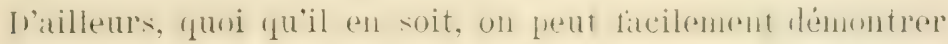
que le mole de la gatstrulation, et le point ciu l'invarination de la blastula pent aroir lieu, doirent être à peu près constants, pur une meme expece d'animatux. si l'on consilere, en eflet,

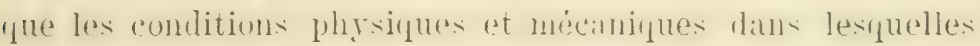
se trouve l'agrtagat cellulatre au moment he lat gastrulation,

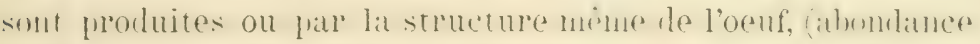
de deutoplasma, présence ou absence de la membrane, etc.), ou bien encore par le mode de segmentation, on comprend sans difliculté que, ces structures étant constantes pour une mème espèce d'animaux, les conditions de la gastrulation seront, elles aussi, constantes.

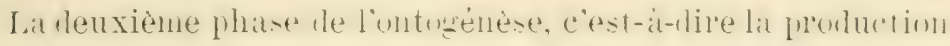

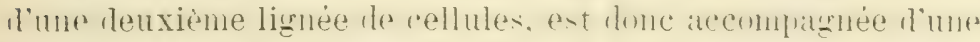
différenciation histolonique at dune diffërenciation morpho-

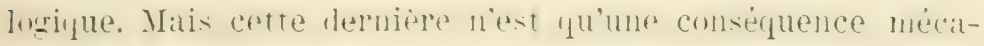
nique de la prolifération cellulaire et du mode par lequel celle-ci s'accomplit. Si l'on roit done que la gastrulation est une phase ontrgénétique commune it tous les Mritaznaires. c'est que, dans tous ces animaux, la première lignée de cellules est suivie par la production d'une deuxième lignée; si,

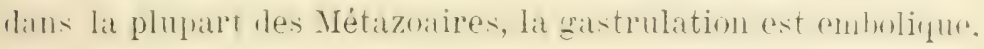
c'est que, dans ceux-ci, les conditions mécaniques réglant la formation de la deuxième phase de l'ontogénèse sont à peu près les mêmes. Ici encore, non moins que dans la formation de l'agrégat cellulaire de segmentation, on doit roir dans lat

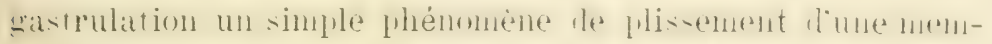
hrane obéiscant anx lois grènérales de la mécaniqur, qunique la cause de ce plissement, la proliferation cellulaire, soit naturellement un phénomène exclusivement biologique.

Il nous reste maintenant à démontrer que la prolifération cellulaire et les diffërenciations histologique et morpholoniqur. le cette leuxieme phase dr l'ontugénèse sont lucalisées dann 


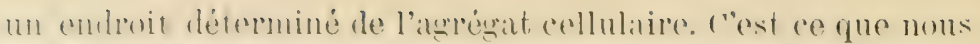
intulieroms particulierement dans le chapitre suivant. Cependant, je peux, dès à présent, faire remarquer que la locitlisation do ces phénomènes est me les conséquences les plus simples et les plus naturelles de mon interprétation de l'ontogénèse, c'est-à-dire du développement monodique.

11 suflit d'examiner lat constitution de l'agrégat cellulaire 16" semmentation, telle que je l'ai leprexentẻe dans les fogmes précédentes, pour comprentre que, si la nouvelle lignée de cellules est prorluite par la prolifération llu blastomère arriré ¿ la phase limite de l'évolution de l'oeuf, du blastomère $p$ ) dans notre exemple, comme ce blastomère possèle dans l’atgrrégit une place déterninée, quelle qu'elle soit, les cellules issues de ses dirisions devront naturellement occuper dans l’arrégat la mème place et les parties avoisinantes de celle-ci.

Lat prolifération cellulaire et la difrérenciation histologique ont donc une locališttion déterminée dans l'agrégat cellulaire, firce quelles se forment lä oü se trouve le blastomère arrivé ¿ la phase limite de l'évolution de l'oenf'. Mais il n'en est pas ftr méme pour la locilisation de la diflérenciation morpholorripue. ('elle-ci n'est pas hien érilente, parce que le plissement yui lat carratérise ne pent pas intéresser un point seul, mais tout l'agrégat cellulaire. Cepembant, la cause du plissement, la prolifération cellulaire, est sans doute localisée.

En lésumé, nous conclurons:

$1^{\circ}$ Les cellules dérivant de la segmentation de l'oeuf préparent, arec leurs produits de sécrétion, le milieu interne ale l'agrégal cellulaire qu'elles forment.

$2^{\circ}$ Les substances de ce milien interne servant de nour-

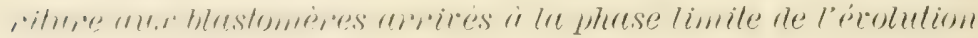
de l'oeuf, produisent une deuxiène lignée de cellules caraclé-isant la deuxiène phase de l'ontogénèse.

$3^{\circ}$ La probiose des cellules de la première lignée est la cruse de la jooduclion de la deuxieme lignée. In peu de 


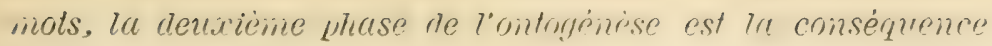
de la phase précédente.

$4^{\circ}$ La production des cellules de la denxième lignée est.

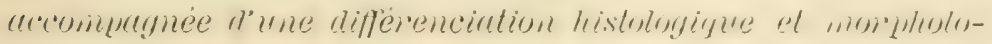
gique.

$5^{\circ}$ La différenciation morphologique, it saroir la gastrulation, n'est qu'un phénomène exclusivement mécanique.

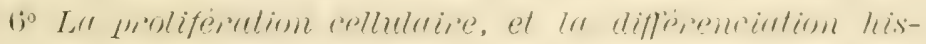

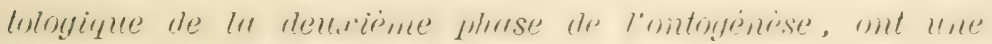
localisation déterminée dans l'agiégal cellulaive.

$7^{\circ}$ Celle localisation est une conséquence directe du développement monodique. 


\section{Chapitre $\mathrm{X}$.}

\section{L'origine de la symétrie rayonnée.}

SOMMAIRE: La production des lignées de cellules do la deuxième phase ontogénétique - La localisation de ces différentes lignées de cellules - L'asynchronisme accéléré et ses conséquences - L'asynchronisme ralenti et l'origine de la symétrie rayonnée - Conclusions générales - Résumé.

Afin de mieux fixel nos idées, revenons maintenant ì notre सxemple et appliquons-y ce que nous renoms d'établir dans le chapitre précédent.

Supposons done que le blistomere $\mu^{\prime}$ (firr. li3), se nourrissant des substances n produites de lit probiose des autres blastomères, soit capable de suirre une autre érolution dont les phases soient $l^{\prime}, c^{\prime}, d^{\prime}$ etc.... p p et supposons encore, pour plus de simplicite, que lastnchronisme de division de ces phases soit accéléré et que, tout comme nous l'arons supposé pour les phases d'érolution de l'oeuf, la durée de la période assimilatrice de ces phases soit:

\begin{tabular}{|c|c|c|c|c|}
\hline$\nu$ & $\gg$ & $b^{\prime}$ & » & l h. 55 \\
\hline$\gg$ & 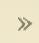 & $c^{\prime}$ & » & 1 h. 50 \\
\hline$»$ & $\gg$ & $l^{\prime}$ & » & $1 \mathrm{~h} .45$ \\
\hline 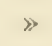 & $\gg$ & $e^{\prime}$ & 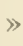 & I h. $40^{\prime}$ \\
\hline$»$ & $\gg$ & $f^{\prime}$ & » & $1 \mathrm{~h} .35$ \\
\hline » & $\gg$ & $g^{\prime}$ & 》 & l h. $30^{\prime}$ \\
\hline$»$ & $\gg$ & $h^{\prime}$ & $»$ & 1 h. 25 \\
\hline$»$ & $\gg$ & $i^{\prime}$ & » & 1 h. $20^{\prime}$ \\
\hline$\gg$ & $»$ & $k^{\prime}$ & » & 1 h. $15^{\prime}$ \\
\hline » & $»$ & $l^{\prime}$ & $》$ & 1 h. $10^{\prime}$ \\
\hline 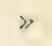 & $》$ & $m e^{\prime}$ & 》 & 1 h. $5^{\prime}$ \\
\hline$»$ & $\gg$ & $n^{\prime}$ & » & $1 \mathrm{~h}$. \\
\hline " & $»$ & $o^{\prime}$ & » & 0 h. 5 \\
\hline
\end{tabular}


Aussitôt que le blastomère pr aura pris naissance par division

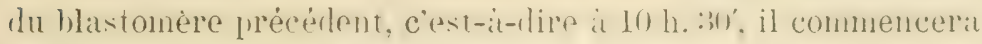

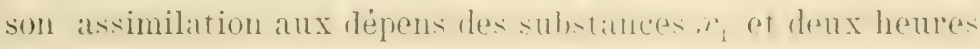
après, a 12 h. 30', il se divisera en deux cellules $7{ }^{\prime}, c^{\prime}$.

Mais l'assimilation du blastomère p ne pourra empêcher' l'assimilation des autres blastomères qui se trourent encore à des phases intermédiaires de l'évolution de l'oeuf. Pourru

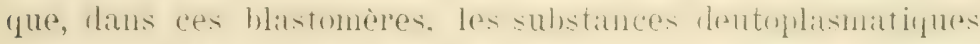

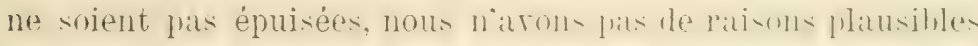

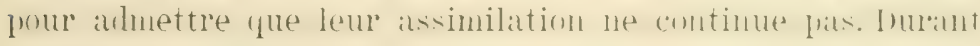
la période assimilatrice du blastomère p, les autres blastomères se diriseront done régulièrement, suivant le développement monodique et le rithme de segmentation que nous avons supposé.

A 12 h. 30', plusieur's autres blastomères auront done atteint la phase linite ${ }^{\prime}$ et, naturellement, eux ausi commenceront leur assimilation aux lépens des nouvelies substincess a frur se diviser en $b^{\prime}, c^{\prime}$.

La production de ces cellules de la ¡e lignée, qui s'entre-

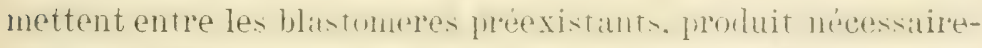
ment un déplacement de crenx-ci: natis connese cette froduction est accompagnée doune dininution less substanees le lat cavité

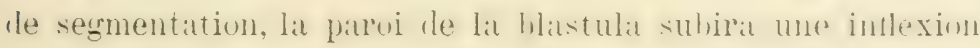
qui sera le commencement du plissement aboutissant à la gastrulation embolique.

Ce plissement commencera donc à $12,30^{\prime}$; mais il sera naturellement trés peu semsible. n'étant froduit que pal lit lormation de deux cellules seules.

Mais à 11 h. 25', le blastomère o (fig. 13) né à 10 h. $30^{\prime}$ se sera transformé en $p$ sans se diviser et commencera son assimilation aux dépens des substances $\alpha_{1}$. Deux heures après, à 13 h. 25', il se dirisera donc, lui aussi, en $\ell^{\prime}, c^{\prime}$. Le plissement commencé ì 12 h. $30^{\prime}$ subira donc une pause entre 12 h. 30' et 13 h. 25 , pour continuer it cette heure. Lais dans ce cas aussi, il ne sera que très peu sensible. 


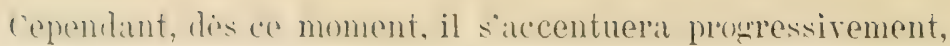
car lo blastomère $m$ (fig. 13) né ì 9 h. 30' se sera divisé à li h. 3is en deux blistomeres h, o. Ce dernier se tratusformera en $l$ it 11 h. 30' et deux heures après, a 13 h. 30', se divisera it son tour en $l^{\prime}, c^{\prime}$.

De même, le blastomère $n$ se dirisera en $0, p$ à $11 \mathrm{~h}$. 35', et

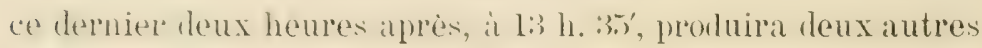
cellules $l^{\prime}, c^{\prime}$.

Mais sur ces entrelaites, le blastomère ul (tim. 13) né it 9 h. 33; se sera divisé en $n, \circ$ et celui-ci, né à 10 h. 40, se sera transformé en $p$ à $11 \mathrm{~h}$. $33^{\prime}$ et, par suite, il se divisera, lui aussi, en $l^{\prime}, c^{\prime}$ ì 13 h. $35^{\prime}$.

Dès ce moment, les blastomères arrivant à la phase $p$ et, par suite, se divisant deux heures plus tard en $b^{\prime}, c^{\prime}$, se succèderont sans cesse séparés l'un de l'autre par un laps de temps qui ne sera pas plus long que 5 minutes. Par conséquent, le plissement s'accentuera peu it peu et continuera sans d'autres pauses.

Mais à $14 \mathrm{~h} .20$, la cellule $c^{\prime}$, née de la division du premier

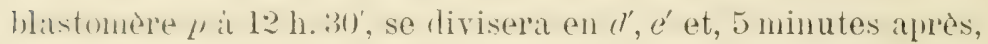
se divisera aussi la cellule $b^{\prime}$ en $c^{\prime}$, $d^{\prime}$. Dès ce moment, le plissement saccentucra plus encore, parce que a l'action des noureaux $p$ paraissant dans l'agrégat cellulaire et donnant lieu à autant de nouvelles lignées cellulaires $b^{\prime}, c^{\prime} \ldots p^{\prime}$, s'ajoutera l'action des proliférations des cellules de ces lignées.

Enfin à 2l h., c'est-î-dire 10 h. 30' après l'apparition du

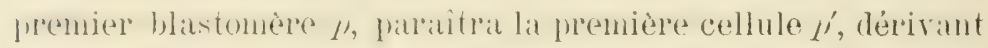
de la division du blastomère p né à $10 \mathrm{~h}$. 30', et des cellules issues de la division de celui-ci. Mais 55 minutes après, ¿t 21 h. 5̌s', paraîtra une autre cellule $\eta^{\prime}$ de la lignée des cellules dérivée de la division du blastomère $p$ né à 11 h. 25', à linpulle, le o en is minutes, feront suite les apparitions des cellules $q^{\prime}$ issues des autres blastomères $p$ nés à 11 h. 30', à 11 h. $355^{\prime}$ etc.

Pendant que les premières cellules $y^{\prime}$ paraissent succes. 
sivement, dérivant de charque biastomere l/, dautres cellules l' dèriveront de la division de lat minne lingée de cellules issues du blastomère $p$ né à 10 h. 30 '.

Si maintenant on considère qu'au fur et à mesure que lir segmentation progresse, le mème phénomène se probluit jour les autres blastomères $f^{\prime}$ nés après, on comprendrat facilement qu'en peu de temps les cellules a lat phase fi deviemdront très nombreuses et que l'agrégat cellulatire prendra une constitution tellement complexe que la représentation graphique en devient presque impossible.

Lai deuxième phase de lontogénése, la production de la deuxipme lignée de cellules, se prorluira donc tout conme la première, à cette différence près que, tandis que les cellules constituant ia première phase ne forment qu'une seule lignér. parce qu'elles dérivent iontes de la dirision d'une seule cellule, l'oeuf, les cellules de la deuxième phave le l'ontogénèse aplartiendront it plusieurs lignées, c'est-it-lire à autant de lignées qu'il y a de hlistomères de la première lignée arrivés it la phase limite, point de départ pour leur nourelle érolution.

Il est d'ailleur's bien érident que, comme cette nourelle éroIution est déterminée, d'une part, par la nature des blastomères a la phase linite - dans notre exemple, des blastomères $p$ - et, d'autre part, par la présence des substances nourrissantes de la cavité de segmentation - dans notre exemple, les substances. ${ }_{1}$ - quand celles-ci seront épuisées, lérolution derra forcément s'arrêter. Par conséquent, comme les nourelles lignées de cellules de la deuxième phase de l'ontogrenese ne sont pas toutes contenporaines, mais successires, une partie seulement pourra arriver it la thase limite p' de leur nourelle érolution arant l'épuisenent des substances nurrissantes $r_{1}$. Toutes les autres se troureront éridemment arreties dans une des phases intelmédiaires de leur érolution.

Il faut néannoins remaryuer que lat localisation de toutes ces lignées de cellules sera à peu près la même. En efret, comme les blastomères qui arrirent successivement a la phase 
j) sont contigus l'un it l'autre, ainsi que le lecteur pourra facilement s'en convaincre par l'examen les figures précédentes et par le mole du déreloppenent monodique, il arrirera necessitirement que toutes les nouvelles lignées de cellules seront, elles aussi, contiguiss et ne formeront, pour ansi dire, qu'une lignée seule au point de vue morphologique.

Ial constitution de cette lignée composée sera même typiquement analogue a celle de la premiere phase, sauf, bien entendu, le nombre plus grand des cellules. Mais ce qu’il importe surtout de remarquer, c'est que, dans la constitution de cette nouvelle lignée, nous pourrons constater la présence de cellules homonymes contemporaines, tout comme dans l'atgrégat de segmentation, el, par conséquent, cette symétrie bilatèrale que j'ai démontrée ailleurs comme étant une conséquence du déreloppement monodique.

Or, c'est précisément sur l'origine de la symétrie, c'estit-dire sur les rapports entre la symétrie de l'agrégat cellulatre de seymentation et la symétrie de l'animal adulte, qu'il faut maintenant s'arrêter quelque peu. Le lecteur pourra se convainere facilement que les symétries rayonnée et bilatérale sont dépendantes de l'asynchronisme de segmentation, et ce ne sera peut-être pas sans quelque étonnement qu'il rerra conment la disposition rayomnée des organes de certains animanx peut trouver dans mon interprétation de l'ontogéenèse une explication qui arrire parfois justu'aux détails les plus minutieux, et cela, sans qu'il soit besoin de recourir a les hypothèses spéciales, mais en analysunt seulement arec attention toutes les consérquences qui découlent naturellement de mon interprétation.

Revenons donc a notre exemple et à nos figures. Cela nous permettra de mieux fixer nos illées et de mieux comprendre ce que je reux demontrer. supposoms que lorsque une cellule de la deuxième lignée arrive à la phase $y^{\prime}$, elle ait acquis une constitution hoplasmatique telle, qu'elle puisse prorluire des cils vibratiles. C"est dire, en d'autres termes, qu'elle a acyuis 
une diflërenciation histologique specialle de cellule ribratile. Supposons encore, afin d'ajouter it ce citratetere histologinue un cartactere morphologique, qute cette rellule vibratile fitsie quelque peu saillie at lat surfice de l'agrégat cellulaire. On obtiendrat alors un petit tubereule ribratile formé d'uns seule cellule.

Aussitôt que d'autres cellules arrireront i cette même phase fi, elles deriendront donce, olles ausio, atutant de petittubereules ribratiles. Mais si ces nourelles cellules sont contigues entre elles et contiguris it lat premiere, elles ne formeront far leur ensemble yu'un seul tubercule d'atutant plus sprand que les cellules sont plus nombreuses. Au contraire, si ces cellules ribratiles paraissent en des points éloigntus l'un de l'autre, c'est-à-dire séparés l'un de l'autre par d'autres cellules intermédiarres, évidenment elles ne torneront pars un seul tubercule vibratile plus grand, mais autant de tubercules qu'il y aura de cellules. Il faut, en somme, se rappeler tomjours que loindividualité morphologinge d'un organe n'est gat: défemdante du mombre des cellules qui le constituent. mais de la position réciproque qu'ont ces cellules.

re print établi, consilérons les conséquences qui lériveront de l'asynchronisme de segmentation accéléré et ralenti.

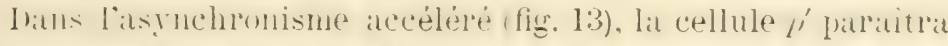
évilemment dals un point quelentque du quatrant A, éestil-dire dians un point du quadrant mime dans lequel se trourait le blastomère $p$, dont est dérivée la cellule $y^{\prime}$. Or, comme je viens de démontrer an commeneenent de ce chapitre que toutes les cellules $f^{\prime}$ res autres lignées seront nécessairement contigupis a celle-ci et contigurs entre elles, il s'ensuirra éridemment que toutes ces cellules ne formeront pas par leur ensemble plusieurs tubercules vibratiles, mais un seul tubercule, dont lat largeur s'accroitra an fur et it mesure que les

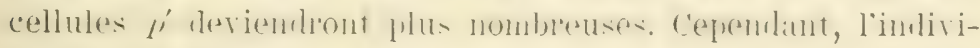
dualité du tubercule sera toujour's unique.

L'agrégat cellulaire présentera donc du coté du quadrant 1 
un tubercule vibratile dont la largeur pourrat néammoins s'accroitre. de maniere a s'étendre aux deux quadrants latéraux $D$ et $C$ et mème, è partie du moins, au quadrant $P$.

Malis dinns le cas de l'asynchronisme ralenti, les choses se passeront d'une manière bien différente.

Nous arons vu que, dans l'așnchronisme ralenti, à 9 h. 55', faubit dams le qualrant a le premier blastomère a la phase I' (lin. 19). ('elui-ci deviendral donc le point de départ d'une nouvelle lignée de cellules $p \ldots \ell^{\prime} \ldots c^{\prime} \ldots p^{\prime}$. En supposant que l'asynchronisme de division de ces cellules soit ralenti, 9 h. 55' après, c'est-i-dire it lo h. 50', paraîtra daus le quadrant d la première cellule $i^{\prime}$ et, par conséquent, le premier tubercule vibratile.

Mais à 10 h. $55^{\prime}$, le blastomère $n$ né à 9 h. et situé dans le quadrant $I^{\prime}$, opposé all quadrant 1 , se divisera à son tour en o, l' et, par' conséquent, paraitra dans ce quadrant le deuxieme blastomère 1 , qui donnera lieu à une autre lignée de cellules $p \ldots y^{\prime}$. A 20 h. 50 ', c'est-ì-dire une heure après l'apparition du premier tubercule vibratile dans le quadrant A, paraîtra donc le 2 tubercule dans le quadrant opposé $P$.

Or, si, dès à présent, nous comparons les résultats de lat localisation de cette différenciation f' dans l'asynchronisme accéléré et dans l'așnchronisme ralenti, nous verrons quelle remarquable différence en découle dans la disposition des organes de l'embryon.

En effet, dans l'așnchronisme accéléré, la deuxième cellule qui arrivera it la phase $j^{j}$ et se transformera en tubercule vibratile, sera necessairement contiguè a la première, ainsi 'fue je lati rémontré. Ces deux tubercules ribratiles n'en formeront donc qu'un seul, d'un volume double du premier.

Mais dans l'asynchronisue ratenti, comme les deux premiers tubercules se troureront dans deux quadrants opposés, et seront sipares far d'alutres cellules intermédiairos, ils formeront inevitablenent denx tubercules nettenent individualisés et bien distincts l'un de l'autre. En outre, l'apparition 
de ces deux tuhereules ne serat pas partititement simultanée. mais le premier paraitrat ditns le plualrant a me heure plus tôt que le deuxième dans le quadrant opposé.

Il faut maintenant remarquer que dans le quadrant $G$ (fig. 19), il y a deux blastomères $n$, dont l'un est né à 9 h. 5', c'esta-dire 5 minutes seulenent plus tarl que le blastonère n du quadrant $P$, et dont l'autre est né à 9 h. 10', et, par suite, 5 ninutes seulement plus tard que le prenier. Ire méme, lans le unarlpant $D$, il y a deux blastomères 12 , lont l'un est contemporain du deuxième du quadrant $P$, car il est né, lui aussi, ¿ 9 h. 10', et dont l'autre est seulement de 5 minutes plus jeune, étant né à 9 h. 15 '.

Lorsque ces quatre blastomères $n, 1$ h. 55 après leur naissance, se diviseront en ", l", chacun des quatre blastomeres donnera lieu à une nourelle lignée de cellules 1 ... pl', et comme les quatre blastomères $p$ sont séparés l'un de l'autre par l'antres cellules interposees, les quatre lignees de cellules qui en dérivent, seront, elles ausi, bien distinctes. Par conséquent, o h. jú après la naissance du blastonère p, paraitront dans lagrégat cellulatre quatre tubercules vibratiles localistes en des points distincts des deux quadrants $G, D$.

A ce moment, l'arrégat cellulare présentera done (; tubercules, dont le premier dans le quadrant t né à 19 h. $50^{\prime}$

\begin{tabular}{|c|c|c|c|c|}
\hline * deuxieme , & $\nu$ & $P$ & $»$ & 20 h. $50^{\prime}$ \\
\hline „troisième & D & $G$ & & 21 h. $40^{\prime}$ \\
\hline - quatrième & » & $D$ & 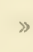 & 21 h. $45^{\prime}$ \\
\hline nquième : & , & $G$ & 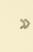 & h. $4 \mathrm{~s}^{\prime}$ \\
\hline xième & $»$ & $D$ & » & $1 \mathrm{~h} .50^{\prime}$ \\
\hline
\end{tabular}

res six tuhercules auront donc une disposition rayonnée et l'arrégat cellularie préventera donc, dès ce monent, mes. métrie rayonnée. Il faut néanmoins remarquer que ces six

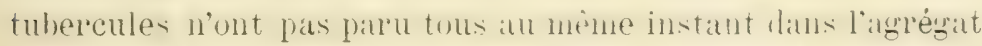
cellulaire. Le quatrième et le cinquième senlement sont rraiment contemporains, itant nés frécisénent it lat mime heure; le troisième, le quatrième et le sixième diffẻrant dans le temps 
de lente apparition de a minntes l'un de l'antre, c'est-it-dire datutant que nous avoms suppesie en diflërep les périodes assimilatrices des cellules. Cependant, si l'on consislere quane diflérence de sminutes senlement dans l'ordre de leur apparition est presque nérligeahle, on peut dire que les tuhercules $3^{e}-6$ e sont à peu près contemporains. D'ailleurs, comme la différence dans le temps de leur apparition est léterminée par la dillërence méme des périules assimilatrices des cellules nn compromdra facilement que, si celle-ci est plus petite, la contempquanéité des apparitions de ces tubercules sera plus érilente, quoique toujours seulement apparente et non reelle: si, au contraire, elle est plus urande, l'ordre de succession dans les apparitions des tubercules deviendra plus manifeste et. toute apparence de contemporaméité disparaitra jresque complètement.

Parni les six tubercules mentionnés, il n'y a que le premier yui ne soit pass lu tout contenporain des autres. Au contraire, il a paru une heure arant le deuxième. Il est donc bien listinctement flus igé que celui-ci et la différence dans l’àge de ces deux tuhercules ext précisément égale à la durée de lat périorle assimilatrice que nous arons supposée au chapitre VII, pour le blastomère $b$. Le lecteur pourra par lui-même se comvincre que, si l'on suppose une durée de la période assimilatrice du blastomère 1 plus gramde on plus petite qu'une herule, la difference entre les temps de l'apparition du batstomère $p$ dans le quadrant $A$ et dans le quadrant $P$, et, par suite, la diffẻrence entre les temps d'apparition des deux mentiers tubercules variera de la mème manière et sera toujomrs égale it la durée de la période asimilatrice du blastomère $b$.

l'ar conséquent, nums jourons conclure que le monent d'apfarition th prenier tubercule sora toujours nettrment séparé

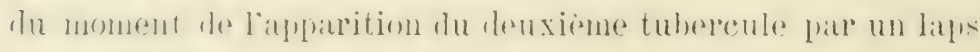
de temps plus ou moins long, égal à la durée de la période assimilatrice de lat phate immerliatmuent successive à l'oeuf, 


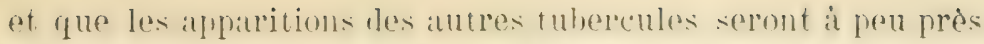
simultanées, n’étant separées l'une de l'autre que par un laps

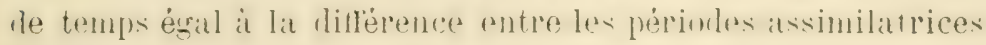
des différentes phases de l'évolution de l'oenf.

Je dois néammoins faire remarquer que ces conclusions ne leurent itre appliquées a tons les antres cas qu'en tenant un compte exact de tous les facteurs qui penvent concourir it les morlifier. "est ainsi, par exemple, que si lon smpuse que les diffërences entre les périorles assinilatrices des diflërentes phases de l'érolution de l'oeuf ne soient pas toutes égạles, ou bien encore, que le rythme de division des cellules de la deuxième lignée ne soit fas éral a celui des cellules de la premiere lignese, conme je viens de le supposer pour plus de simplicité, les conclusions ens seront quelque freu motifiées, tambis que le principe dont elles découlent restera exactement le même.

Mais ce qu'il y a de surprenant dans les conséquences dérirant de cette interprétation. cerst la coincidence parfaite yui existe entre ces résultats tout-it-lait thérniques et les données

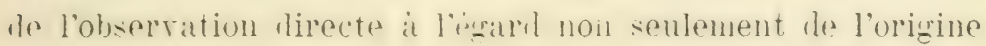
de la symétrie rayonnée des Coelentérés, mais aussi de l'ordre d'apparition des organes rle ces animaux. Il suffit, par exemple, d'examiner borde d'apparition des tentarules, tel

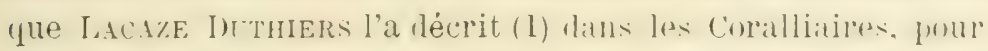

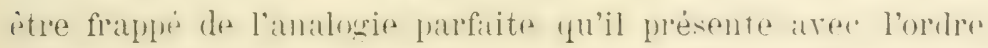
de l'apparition des tubercules ribratiles tel que je viens de le démontrer danc notre exemple. On doit surtum pemaryuer lastuchronisme d'aptrition entre le premier et le lenxieme tentacule, et la presque contemporanéité des autres.

On pourrait démontrer, en suivant toujours la mème méthorle, que d'autres tubercules derratient ses produire aree une

(1) Lacaze Dutmers H. - Développement des Coralliaires in: "Arch. de Zool. expér. ". Tome I, 1872, pp. 289-396. Voyez aussi: Délage et Hérocard - Truile de Zoolegie concrete. Tome II, 2 Partie. Paris, 1901. 


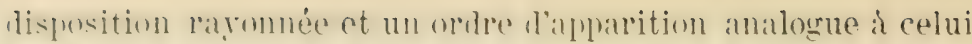
que je riens dexposer. Matis, pour celal, serait néessaire un agredgat cellulare constitue dun mombre de cellules bien plus grrand que celui de l'atrégrat de lit ligg. 19. Or cet agrégat, qui. d'ailleurs correspondrait évidemment mieux aux agrégats réels tels que nous les royons dans les observations embryoloriques, présenterait une telle complexité que sa représentation graphique deviendrait presque impossible.

D'autre part, il faut remarquer que le nombre plus grand des cellules peut, par lui-meme, coeteris puritus, fitre la cause d’un plus grand nombre de locilisations des différenciations morphologiques. En ellet, si le nombre des cellules de l'agrégat est plus petit (et ce nombre, nous l'avons ru, dépend de la phase limite de l'érolution le l'oenf et du rythme de dirision) le nombre des blastomères donnant lieu aux lignées de cellules de la deuxieme phase seri, lui aussi, plus petit et, par suite, les différenciations morphologiques, dans notre exemple les tubercules ribratiles, seront moins nombreuses.

En outre, comme nous arons ru que l'individualité des diffërenciations morphohgiques est dépendante de lat présence dans l'agrégat cellulatre de collules intormédiatres séparant les hastomeres somches des nurelles lignies de cellules, dans notre exemple les blastomères $\psi$, il est érident que, si les cellules de lagrégat sont plus mombrases les groupes des cellules de sépiration seront plus aramds et plus nombreux, tandis que si les cellules sont moins nombreuses, il pourra arrirel plus facilement que les blastomeres, souches des nouvelles ligrnés: de cellules, sient contigus et, par conséquent, 'fur les lignies de cellules issues d'eux ne donnent pas lieu it une autre dillérenciation morphologique indivisualisée, mais ne fissent yuaccroitre tout simplement la diflérenciation produite par la limée de cellules issues du blastomere contigu. De cette manière, on obtiendrait une seule au lieu de denx ou plusieurs différenciations morphologiques.

Enfin, je ferati mene remarquer que, lans les considérations 
que je riens de faire, je n'ai pas tenu compte, pour plus de

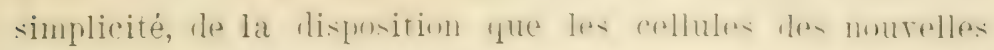

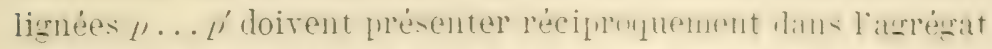
cellulatre. En supposant yur l'atruchronisne do division de ces cellules soit ralenti, tout comme colui des collutes do lat

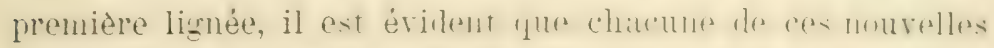

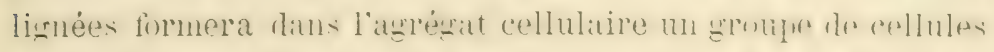
a des phases différentes intermèliaires entre piet fi, lont la

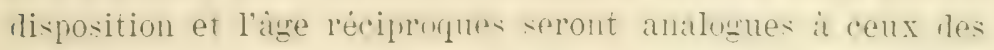
cellules de la fremiere lignée, comme nous l'arons rentrésenté dans la firr. 19. Dans ce cat, on peut fitcilement fréroir que lay-

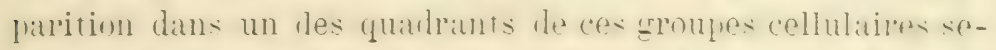

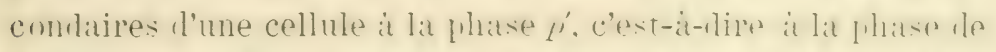

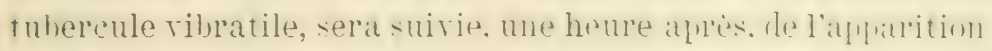

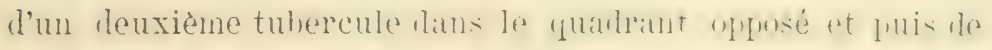
l'apparition d'autres tubercules dans les autres quadrants.

De cette manière, les phénomènes se compliquent de plus en plus et la complication devient telle qu'il est impossible

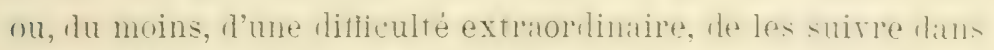
leur's létails, et, it plus finteraison, le les remrésenter cratphiquement par des figures.

Je me borne donc à conclure d'après ce que je riens de démontrer. que la cause ontogenétinue le la synétrie ritronnes? est l'asychronisme ralenti le la sermentation: que l'ugrtigat cellulaire de sesurentation présente dans sa constitution nrimitive une stmetrie hilatérale: que cette smuétrie lilittralte se rérèle encore dins l'apparition des ntlërenciations, mais qu'elle di-parait apparemnent et se tran-forme en une symétrie ranonnée, si lon tient annpte semlenent de la lomisation de ces différenciations.

Telles sont les conclusions sur l'origine de la symétrie rayonnée que je riens d'établir d'après un raisonnement logique et exclusirement thèrinue. en partant le mon interprétation de lontogendse. Iqukervation des fuits monerots dira si ces résultats theriques concundent arec les monées des 


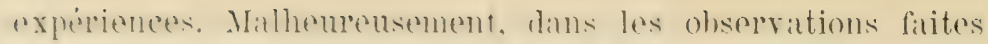
jusqu’jei. on n'a pas temu compte des facteurs qui probluisent, at mon avis, la symétrie rayomnée. J'espère que ce travail pourlat frovouner dans ce but, des recherches biologiques; mais je ne suis quels en seront les résultats, à cause des difficultés trex grlunkes quelles presentent. Cependiunt, la vérification de la conclusion fomdamentale ne sera pas diflicile. Je crois que les Biolonistes ponront assez aisément rérifier si, dians les animanx at strmetrie latromée, l'asynchronisme de serrmentation est rilenti, c'ext-it-rire si la segmentation générale le l'venf sulit un ralentissenent i mesure qu'elle progresse.

1)ailleurs, le lecteur complendra partaitenent que, daus ce trivali, je ne puis ne proposel d'autre but que d'indiquer le morle grenéral d'interpuétation des phémomènes de l'ontorénèse. Eridemment je n'ai fras la prétention d'expliquer tous les cas particuliers si nombreux et si variés que la nature mons presente. W'abord, la plupart de ces cas ne pourront être interprétés ou résolus que lorsque les commaissances sur leur nature intine seront plus profondes et plus minutienses; en second lieu, leur solution rorra tre fate pour chacun d'eux en particulier, en tenant exactement compte de tous les facteurs dont elle peut dépendre.

Mais je crois que, dans la base de mon interprétation, c'estit-dire dins le développement monodique et dans l'asynchronisme dra segmantation, les Binlogistes gromront trouver toutes

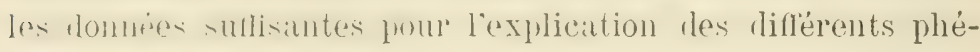
mmònes ontugénétiques, quelque rariahles qu'ils puissent ôtre, pourvu, bien entendu, que l'on tienne compte de toutes les romritions physigues, chimiques et mécaniques daus lesquelles ils s'accomplissent.

En résumant, nous conclurons:

$1^{\circ}$ Si l'asynchronisme de segmentation de l'oeur est accéléié, la première différenciation se localise ans un seul joint de l'agrégal cellulaire de segnentation. 
$2^{\circ}$ Si l'asynchronisme de segmentation est ratenti, la jine-

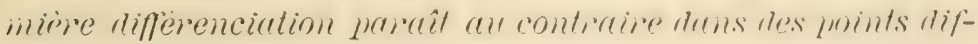
férents de l'agrégat cellulaire.

$3^{\circ}$ Ces points ont une disyrosition rayonnée: d'oir iésulle la symétrie rayonnée de l'organisme futur.

4. La symétrie rayonnée, considérée au point de vue ontogénétique, est done l'efret de l'asynchromisme de sèmentation ralenti.

$5^{\circ}$ Les apuaritims.s des différenciutions dans les différents points de leur localisation dans la symétrie rayonnée ne sont pas parfaitement simultanées, mais successives. 


\section{Chapitre XI.}

\section{L'origine de la symétrie bilatérale.}

SOMMAIRE: Effets possibles de la probiose des cellules de la première lignée

- L'asynchronismo accéléré - La polarité de l'ombryon - La symétrio bilatérale - Causes de cette symétrio - Préoxistence do la symótrio de l'embryon dans l'oeuf - Symétrio de l'agrégat cellulaire de segmentation ot symétrie de l'embryon - Rapports entro lo plan de symétrio ot les dux promiers plans de segmentation - Epoque de l'apparition de la symétrio bilatérale - Résumé.

La supposition du tubercule vibratile que je viens de faire au chapitre précédent n'ayant d'autre but que rle mieux concréter uos idées sur l'individualité des différenciations morphologiques et sur la localisation de celles-ci, nous pouyons maintenant l'atbandonner, et revenir ì l'examen des faits tels qu'ils se présentent dans la réalité.

La deuxième phase de l'ontogénèse est donc caractérisée par la probluction done deuxiene lignée de cellules, et comme cette lignée dérive du blastomère arrivé à la phase limite de l'évolution de l'oeuf et de la nature des substances sécrétées par les cellules de la première lignée, il y aura évidemment autant de ces nouvelles lignées qu'il y a le blastomères it la phase limite, et la production de ces lignées se continuera

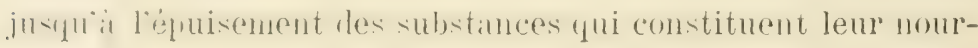
riture.

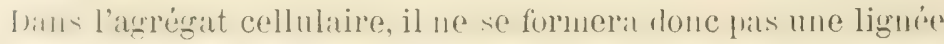

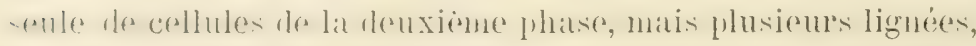
constituées d'après le même type, c'est-it-dire caractérisées

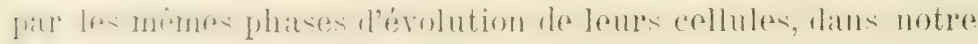
exemple par les phases $p \ldots b \ldots c \ldots l i$. 


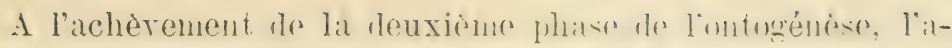

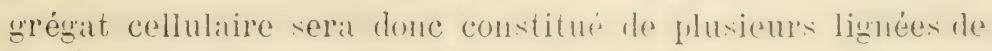
cellules de la deuxième phase: $p$... p' et d'un certain nombre

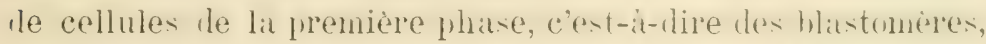
lespuels n'ayant pars encore atteint la phase linntep de lérolution de l'oeuf se trouvent encore dans une des phases intermédiaires rle celle-ci.

Si l'asyuchronisme de segmentation était ralenti, les nou-

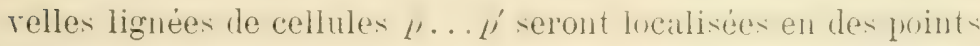
diflërents de lagrégat cellulaire arec une srmétrie ratomnée, comme je l’ai démontré au chapitre précédent. si, a contraire, l'asynchronisme de segmentation était accéléré, toutes les nourelles lignées de cellules $\ell_{\ldots} . . h^{\prime}$, quel qu'en soit le nombre, seront localiséer dans un seul endroit de l'agrégat cellulaire.

Faisous abstracticu pour le moment de la localisation de ces cellules, et examinons seulentent quelles sont les conséquences pouvant dériver de leur production.

bidemment, les celluless de la leuxiène lignée, tout comme celles de la première, produmont, pembant leur érolution et leur assimilation, des substances preciales le sécrétion qui, s'accumulant it l'intirieur le l'agrégat cellulaire, constitueront le milieu interue caractéristique de la deuxième phase.

Or, de deux choses l'une: ou bien ces substances ne pourront servir de nourriture it aucune des cellules constituant l'agrégat cellulaire, ou bien elles pourront servir de nourriture ì quelques-unes de celles-ci.

Dans le prenier cas, il est évilent que le dérelopuement sera forcément arreté. L'enbryon ne progressera pas ultérieurement dans son érolution ontogénétique. Nons n'arons donc pas à le considérer.

Inans le deuxiène cas, an contraire, la nutrition de quelquesunes des cellules de l'agrézat aux dépens de ces substances de sécrétion entran̂era éridemment une nourelle prolifiration cellulaire et en meme temps une nouvelle dillërenciation chimique. C"est ce que nous derons précisément exaniner. 
Il fiut remarquer arant tout que, lorsque ces nouvelles substances se formeront, il y aura dans l'agrégat cellulaire deux sortes de cellules: les cellules de la première lignée et celles de la deuxième lignée.

Or, parmi toutes les collules de la première lignée, les blastomères, quelunes-mes seront arrivées ì la phase limite de l'érolution de l'oeuf et celles-ci, i leur tour, suivront la nouvelle érolution $p . . . p$; les autres se troureront encore dans une des phases intermédiaires.

Si donc on suppose que les nouvelles substances de sécrétion puissent servir à l'assimilation d'autres cellules, il n'y a que deux cats possibles à considerer: ou bien ces substances ne peurent servir de nourriture qu’aux cellules arrivées à la whase linite caractéristique de la deuxième lignée de cellules, - dans notre exemple aux cellules $y^{\prime}$, - ou bien ces substances peurent servir de nourriture a certaines cellules de la première lignée it une phase quelconque de leur érolution. C'ela dépendra évidemment de la nature spéciale de ces substances en rapport arec la constitution bioplasmatique des cellules réagissant avec elles.

Considérons donc ces deux cas possibles.

Le premier cas, et c'est le plus simple, sera parfaitement analogue ì celui que nous arons considéré lors de la production de la deuxieme lignte de cellules. De méme que les substances sécrétées par la probiose des cellules de la première lignée fournissent la nourriture aux cellules de la phase limite de cette lignée et proroquent ainsi la prolifération des cellules de la deuxime lignée, de même, les substances sécrétées far les cellules de la deuxiène lignée, servant de nourriture aux cellules arrirées a la phase linte de cette même lignie, en provorquent la prolifëration et, par conséquent, la formation d'une trosirme lignée de cellules que nous pourons représenter par $y^{\prime} . . . y^{\prime \prime}$. Ici encore, nous arons un exemple de l'importance de la probiose dans les phénomènes ontogénétiques. La probiose des cellules de la deuxieme lignée serait donc la cause 
de la formation d'une troisieme ligné dr cellules. Celle-ci caractériserait la troisième phase de l'ontogénèse.

En poursuivant toujour's le même mode de raisonnement, nous pourons ficilement comprendre comment lat troisiemer lignée de cellules peut tomner lieu it une quatriène lignée, et celle-ci à une cinquième etc., où la probiose des cellules d'une lignée est la cause de la formation de la lignée suivante. Le phénomène ontogénétique consisterait donc, dinn ce cas, dans une série ininterrompue de lignées te cellules, se succédant dans un ordre déterminé car il est évilent qu'une lignée quelconque de ces cellules ne pourra se produire que si elle est précédée d'une antre lignée, limpelle, aree les suhstances sécrétées full ses cellules, puisse prérarer le milieu interne favorable au réveloppement de la lignée suivante.

Ie cette manière, on peut déjà comprendre parfaitement comment les différentes phases de l'ontogrnése, caractérisées par autant de lignées de cellules, sont intinement liées l'une à l'autre dans un ordre déterminé, et comment une phase ontogénétique quelconque ne peut itre atteinte qu'en passint par les phases intermédiaires.

Ainsi, d'une façon très simple et très logique, nous pourons obtenir une explication scientifique du phénomène le plus inportant et le plus caractéristique du déreloppenent enulnyonnaire, à savoir la succession des diflérentes phases par lesquelles l'embryon doit forcément passer atrant d'arriver it sa constitution définitire. Nous rerrons d'ailleurs, dins une autre partie de ce travill, comment ce phénomène ontogénétique, considéré an point le rue phylogénétique, peut nous donner un explication scientifique très clairo et rationnelle le la loi biogénétique fondamentale.

Mettons maintenant la prorluction le cette troisième lignée de cellules en relation avec l'astrnchronisme de sermentation accéléré ou ralenti, et nous rerrons quelles conséquences en découlent.

Dans l'asynchronisme accéléré, toutes les cellules py, souches 
des ligniess de lat deuxieme phase ontogénétique, sont localisées dins $u n$ senl et mime endroit de l'atgrézat cellulaire, ainsi que je l'at démontré. P'all conséquent, les cellules h', phase limite de lit denxieme lignee, selont, elles aussi, localisées dans un seul et mime emlroit de l'arrégat cellulaire: car toutes les cellules qui arriveront sucersirement it la phase limite $p^{\prime}$, seront contiguës entre elles. Les cellules $y^{\prime} \ldots p^{\prime \prime}$ de la troisieme lignte seront donc, elles aussi, of pour la même raison, locialisces dins un seul endroit de l'algrigat, ot on doit nécessarement arriver a la même conclusion pour la localisation des lignées des autres phases successives de l'ontogénèse.

Evidemment, dans ce cas, l'enbryon issu de la segmentation de l'oeuf ne présenteria las une réritable symétrie. Comme les différentes lignées de cellules se succèdent l'une à l'autre arant que les lignées précédentes aient achevé leur formation et à plus forte raison, avant qu'elles aient dispar'u complètement, il s'ensuivra naturellement qu'i une époque quelconque de son déreloppenent, le corps de l'embryon résultera formé d'une série de lignées de cellules, lesquelles pourront, par leur constitution et par leur disposition, représenter des différenciations histologiques et morphologiques diflërentes. Nais comme ces différenciations sont différentes l'une de l'autre, quelle que soit la disposition qu'elles pourront aroir, l'embryon ne présentera ni une symétrie bilatérale, ni une symétrie rayonnée, mais tout simplement une polarité. Et cette polarité sera d'autant plus marquée que les diverses diflérenciations présenteront une disposition en série. Le corps de l'embryon nous paraitra alol's comme constitué par une série d'organes. Nous avons des exemples d'une constitution semblable dans les colonies polymorphes des siphonophores (1).

(1) Le lecteur doit iei renarquer que je ne parle pas do la symótrie des partios de l'embryon, mais de la symétrio de l'embryon tont entier. 1)ans phisienrs Siphonophores, par exomple, los individus de la colonie ont uno symétrie rayonné; mais la colonie tont entière, représentant l'embryou issu de l'oenf, n'a pas de véritable symétrio. 
Dans l'asynchronisme ralenti, an contraire, comme la disposition des blastomères l" présente dans l'arrrégat une symétrie rạonnée, la mẻme disposition sera présentée éridenment par les cellules $p^{\prime}$ et les cellules $y^{\prime \prime}$, ainsi que par les autres cellules issues de celles-ci. L'embryon conservera donc toujours la symétrie rạonnée qu'il a acquise dès la deuxième phase de son développement.

Considérons maintenant le deuxième cas. Supposons que les substances sécrétées par les cellules de la deuxime lignéce puissent entrer en réactions d'assimilation arec les cellules de la première lignée.

Nous arons vu que, dans le déreloppenent monodique, tous les blastomères arrivent tour a tour a la phase limite de l'érolution de l'oeuf. Mais lor'sque quelques-uns y sont dejai arrives et ont commencé, par suite, leur nourelle érolution $p . . . y^{\prime}$, d'autres blastomères se trouvent encore ì des phases intermédiaires de l'évolution. Quel que soit donc le moment ou les nourelles substances $x_{2}^{i}$, produites par la sécrétion des cellules de la deuxième lignée, paraissent dans le milieu interne de l'agrégat cellulaire, il y aura, à ce même instant, des blastomères à la phase limite $p$ et d'autres se trouvant encore diuns quelques-unes des phases précédentes.

si donc l'on suppose que ces nouvelles substances puissent servir de nourriture it quelques-uns de ces blastomères qui n'ont pas encore commencé l'évolution $\not$....p', ceux-ci, en présence de ces substances nourrissantes, commenceront leur assinilation et, par leur's proliférations successives, toujour's suivant le développement monodique, produiront une autre limnée cellulaire, tout comme les hlastomères $/ 1$ ont pronluit la deuxième. Et cette nouvelle lignée sera constituée, elle aussi, d'une série de phases qui la caractériseront.

Mais comme les phases caractéristiques de chaque lignée cellulare sont dépendantes, l'une part, de la constitution hiorplasmatique de la cellule initiale, et, d'autre part, de la composition chinique des substances nourissantes, il s'ensuirra 
uẻcessialement que les phases de la nourelle lignée seront dependantes de la constitution du blastomere que nous suppusons en être le point de depant et des substances nourrissintes, in. Or, quel que soit ce blastomère, quamd même nous roulrioms supposer que ce soit le blastomère f', la nouvelle lignée cellulaire, que nous pourons indiquer tout hrièrement $x_{2} p$, sera nécessairement différente de la première.

C'est ce que d'ailleurs chacun peut comprendre facilement; car si le blastomère $l$, en se nourrissant des substances $\lambda_{1}$ a donné lieu it la lignée cellulaire $\ell^{\prime}$... lí, un autre blastomère l', quoique d'une constitution bioplasmatique identique, en se nourrissant des substances $\alpha_{2}$, différentes de $r_{1}$, derra produire nécessairement une lignée cellulaire différente de la lignée $p \ldots p^{\prime}$.

D'ailleurs, si nous roulons supposer que le blastomère initial de cette lignée cellulaire ne soit pas à la phase $p$, mais à une autre phase quelconque, par exemple, aux phases o ou " ou $m$, la différence entre ces lignées $x_{2} o$, ou $x_{2} n$, ou $x_{2} m$, et la lignée $p \ldots l '$, sera, á plus forte raison, encore plus marquée.

Quni qu'il en soit, il est très sûr que, en tout cas, la nouvelle lignée cellulaire que nous considérons maintenant sera diflérente de la deuxième lignée, ce qui est le point capital pour notre interprétation.

Cela étant posé, considérons maintenant les conséquences yui dérivent de la formation de cette lignée cellulate dans les deux sortes d'asynchronismes possibles.

Il faut nécessairement tenir compte, dans ces considérations, d'un phénonène dont la connaissance est absolument indispensable pour lit parfaite compréhension de ce que nous allons démontrer.

A l'instant oú nous le considérons, il y a done dans l'agrégat cellulate des cellules appartenant i deux ligneses difrérentos: les hlastomires, de la première lignée et les atutres cellules, de la deuxieme lignée. 
Or, il faut bien remarquer que ces dernires, lors de leur production, en s'entremettant entre les blastonères. en auront produit une séparation, de maniere que, tindis que l'arrégat cellulaine de segmentation, arant leur jroduction, itait constitué de blastomeres tous contigus les uns aux autres sans solution de continuité, ¿t présent cette continuité n'existera plus. Il s'agit, ainsi qu'on le roit, d'un phénomène nécanique très simple et, d'autre part, inéritable, mais dont l'inportance est grande pour les conséquences qui en découlent.

En effet, dans le cas de l'asynchronisme ralenti, les cellules de la deuxième lignée formeront dans l'agrégat cellulaire plusieurs zones radiaires s'alternant arec autant de zones interradiaires constituées de blastomères, ainsi que nous l'arons lémontré. Quels que soient done les blastomères que nous. supposons être le point de départ de la nourelle lignée en question, ces blastomères se troureront nécessairement inclus dans ces zones interradiaires, et les lignées cellulaires qui en dériveront auront naturellement cette mêne disposition.

Après la formation de ces numvelles lignées collulaires, qur nous pourons appeler les lignées interradiaires, l'agrégat celIulatire aura donc acquis une plus grande complication, en tant qu’il sera constitué do cellules de la deuxième lignée, firmant les lignées radiaires et de cellules de la nourelle lignée, formant les lignées interradiaires; mais la symétrie ratonnée caractéristique qu’il arait acquise dès la formation de la deuxième lignẻe ne sera nullement changée.

Il n'en est pas de même dans le cas de l'asynchronisme accéléré.

Comme je l'ai fiit remarquer au chapitre précédent, les cellules des différentes lignées I'..... l' qui, dans lo cas de l'asynchronisme ralenti, forment des groupes à disposition rayonnée et séparés l'un de l'autre par des blastomères, dans le cas de l'asynchronisme accéléré se trourent, au contraire, rimies, ne formant qu'un seul groupe. Lal cause, nous l'arons $\mathrm{vu}$, tient à ce fait, que dans l'asynchronisme accéléré, les 
bianduneres qui atrivent successivement it lat phase point de départ pour la formation des lignées l’... l', sont inévitahlement toms contigus et, par consémuent, toutes les lignées qui en dérivent, doivent itre, elles aussi, contiguès. Ce qui conduit naturellement it la formation l'un seul groupe cellulaire, et, par suite, it ce liat inéritable que l'agrégat le segmentation ne sera pats divisé an plusieurs zones interraliales far l'interposition des cellules des lenxiènes lignées, ainsi que nous l'arons démontré pour l'asynchronisme ralenti, mais qu’il ne présentera une interruption que d'un coite seulement et, plus précisément, du cuté oì se trourait le blastomère arrivé le premier à la phase $p$.

Il s'ensuit que, quelle que soit la phase que nous supposons ìtre le point de départ pour la formation de la nouvelle lignée que nous considérons maintenant, le blastomère qui la reprisentera se trourera nécessilirement d'un coté du groupe cellulaire de la deuxième lignée. De ce coité, se formera donc la nouvelle lignée et elle s'accroitra it mesure que l'autres blastomères arriveront successivement à cette phase.

Mais comne, dans l'agrégat de segmentation, nous l'arons ru, il y a des blastonières homonynes contemporains, il s'ensuirra que cinq minutes apres, an plus tard, une autre lignée illentique se formera et s'accroitra de la méme maniere de lautre coté du groupe de la deuxiène lignée. Par ce mole, celui-ci se troureria compris entre deux groupes cellulaires identiques entre eux et liffërents de lui. Nous pourons done appeler le premier le groupe mindian on imprair et les autres, les groupes bilatéraux ou pairs.

L'agrégat cellulaire présentera donc, dès ce moment, une symétrie bilatérale bien distincte.

Cepemant, il funt bien rematquer que cette stmétrie n'est pas du tout liée a la struétrie bilaterale de langrérat cellulaire, telle que jo l'ai fait ressortir au chapitre IX. J'entends

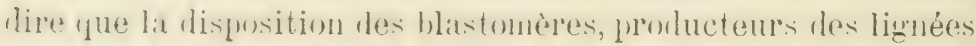
bilatérales, d’un cinté et le l'autre de la ligntrée médiane, n'est 
pas dépendante de la frésence de hatunères homonymes

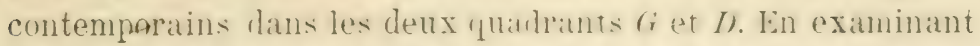
la fig. 13, le lectenr pourra fitcilenent se convainere que dans chacun des quarlants et, par suite, dans le quatrant I' aussi, existent des blastomères homonymes contempmatins, ce qui est d'ailleurs une conséquence directe du diveloppenent monodique. Il recomaitra l'exactitule parfiate de mes déeluctions: ef arrivera it se persualer que londigine le lat stuetrie rayonnée ou bilatirale n'est qu'une conséquence mathénatinue et mécanique des prentises yui constituent la hatse der mon interprétation de l'ontogénèse.

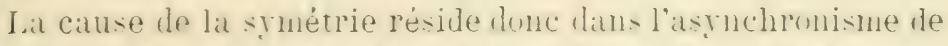
segnentation le loeuf et dans la faculté quiont les hastomères de donner lieu, en rrésence des suhstances spéciales constituant le milieu interne, it des liggnes de cellules diffrentes qui seront le point de départ pour la formation des futurs organes de l'embryon. or, comme cette fiaculé est léplendante de la nature des blastomeres et du nilieu interne. et comme celle-ci dépend, en derniere analyse, he lat constitution de lineuf, il s'ensuit érilemnent upue l'origine le la symétrie dérive de la constitution de l'oeuf. En d'autres termes, la symétrie le l'emhryon est léja préexistante dans linent mime.

Iais cette préexistence n'est pas fondée sur la structure morphologique le lineut. Il n'y a par dans celui-ci une disposition spéciale les particules par mapront at lat sumetrie le l'embryon qui en dérivera ; et bien que, tout récemment,

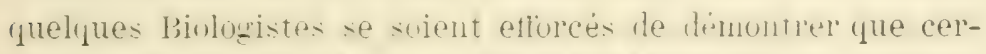
tains oeufs prisentent une smétrie bilaterale dans lentrotructure, je crois neimnoins que, mime ent anmettant que cette srmétrie existe réellement, dle n'a print le relations de causalité arec la symétrie de l'embryon.

Dailleurs, arant admis comme point de départ de mon interprétation, l'isotropisone de lonent, il est évirlent que je ne

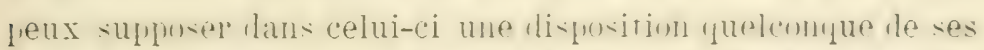
particules arant lit moindre relation arec la structure du 
futur embryon. Palr conséquent, en almettant, ainsi que je l'ai démontré, une préexistence de la symétrie dans l'oeuf, je me réfere exclusivement i la constitution chinique de son bioplasma, a sa constitution bioplasmatique, en écartant rigonreusement toute structure morphologique.

Lil sỵmétrie est donc un phénomène purement mécanique, lont lorigine est néanmoins basée sur la nature des blastomeres et sur leur's propriétés chimiques et, par conséquent, sur la constitution chimique de l'oeuf, dont ceux-ci dérivent.

Il faut done distinguer deux sortes de symétrie: la symétrie de l'agrégat cellulaire de segrmentation et la symétrie de l'embryon. La première, nous l'arons ru, est toujours une symétrie hilatérale, dérirant du déreloppement monodique et consistant dans la présence de cellules homonymes contemporaines dans l'arrégat cellulaire: mais cette symétrie n'est que passagère: car elle est destinée a disparaitre aussitôt que d'autres lirnées de cellules se substituent aux blastomères. Elle n’a l'ailleurs aucune importance pour la symétrie de l'embryon futur. Tous arons vu, en effet, que d'un agrégat cellulaire possélant cette șmétrie bilatérale peuvent dériver des animaux a symétrie bilatérale, autant que des animaux à symétrie rayonnée.

La symétrie de l'embryon, all contraire, ne se forme qu'a l'apparition de la deuxième lignfe cellulaire, s'il s'agit de symétrie rayonnée, ou des lignées paires, s’il s'agit de symétrie bilatérale; mais cette symétrie se conservera toujours: car il est bien évident que, quels que soient les orqanes que nous supposons dériver de ces lignées cellulaires, ces organes auront nécessairement une symétrie rayonnée ou une symétrie hilatirale, c'est-i-dire la mime srmétrie que présentent dans l'agrégat cellulaire ces lignées mêmes dont ils dérivent.

Gest ici que nous pouvons aborder une question très intérossante, qui a homné lieu, dans ces dernières années, à plusieurs discussions. Je veux dire l'orientation de l'embryon par rapport au premier plan de segmentation. 
Revenons donc it la fig. :3, ou le deuxiène plan de segmentation est acheris et ou laugrégat est constitui des quatre preniers blastonères. Ceux-ci sont éridemment compris entre le premier et le deuxieme plan de segmentation, formant ainsi quatre quadrants que nous royons marqués dans la figure susdite par les lettres $A, G, D, P$.

Or, comme le blastomère e du quadrant . 1 est le plus arancé dans l'érolution, il s'ensuivra nécessairement que, même lorsuue les cellules de l'agrégat seront plus nombreuses, le bastomère le plus arance se trourera néammoins foujours contenu dans le même quadrant $A$. Il est rrai que, pendant la segmentation et i cause de celle-ci, les cellules, en grissant les unes sur les autres, pourront changer leur position réciproque; j'admets mème que ce changement se fait, dans la llupart des cas, en mesure plus on moins mante. Mais cela, ainsi que nous le verrons, n'a pas d'importance, et servira même à expliquer plusieurs des faits observés.

Faisons done abstraction le ce déplacement et supposons que le blastomère le plus avancé se troure toujours dans le quadrant A. Evilemment, le blastomère de la phase limite de l'erolution de l'oeuf, (dans notre cas, le blastomère $1 /$ ) se trouvera dans ce mème quadrant, et, par conséquent, la deuxième lignée de cellules occupera, elle aussi, la même place dans l’agrégat cellulaire; car nous sarons qu'elle dérive de la prolifération ultérieure du blastonère $\%$. Or, comme cette lignée de cellules, dans le cas de la simétrie bilatérale, constitue, ainsi que nous renons de le voir, le mroupe cellulaire impair yui sera plus tarl flanqué des deux groupes cellulitires pairs, il en résulte nécessairement que les orwanes qui dériveront de ce groupe impair de cellules seront, eux aussi, inéritablement impairs, tandis que les urganes dérivés des groupes cellulaires pairs seront, eux aussi, pairs. P'al' conséquent, quels que soient les orwanes que nous supposons deriver de la deuxième lignée de cellules, qưils appartiennent a l'extrémité antérieure ou bien à l'extrémite postérieure de l'em- 
hryon, ce qui nata las d'importance, lopientation de l'embryon par rapport au premier plan de segmentation ne sera pas moins determinée: car ces ormanes impail's marpuoront évidemment la direction du plan de simatrie du futur enbryon.

Concrétons mieux nos idées. Supposons que des cellules issues du hlastomere plérivent les cellules qui derront plus tard constituer les parois du tube neural primitif. Celui-ei sera necessairement un organe impair; tandis que, si l'on suppose que les sucs coelomiques dérirent des groupes cellulaires pairs de la lignée intermédiaire, ces sacs se formeront naturellement en nombre pair, d'un côté et de l'autre du tube neural. Celui-ci marquera alors la direction du plan de symétrie de l'embryon, et cette direction, par rapport au premier plan de segmentation de l'oeul, sera la direction même qu'arait le blastomère $p$.

Or, comme le blastomère l' est dérivé de la segmentation du blastomère $e$ occupant le quadrant $A$, il occupera encore une place dans ce même quadrant. Peut-être, à cause de la pression des cellules environnanter pendant la segmentation, il aura subi des déplacements: tantrit il pourra se déplacer vers le premier plan de segmentation, ainsi que je l'ai supposé dans la fig. 13, tantôt il se déplacera vers le deuxième plan de segmentation, tantit encore il prendra une position intermédiatre entre le premier et le deuxième plan de segmentation. Le plan le symétrie du futur embryon sera, par conséquent, en relation très étroite arec ces déplacements. Si le blastomère $f$ a une position intermédiaire entre le premier et le deuxième plan de segmentation, la direction du plan de snmetrie de l'embryon sera, elle aussi, intermédiaire entre ces deux plans; si, au contraire, le blastomère $p$ se trouve tres rapproche du premier plan de segmentation, le plan de symétrie de l'embryon fera avec celui-ci un angle dont la valeur sera très petite et peut-être presque nulle et négli-

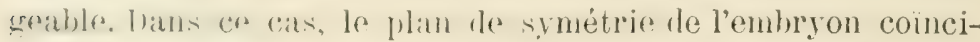
dera, applaremment au moins, arec le premier plan de segmen- 
tation. Enfin, si le blastomère $p$ se troure très rapproché du deuxième plan de segmentation, le plan de srmétrie de l'embryon tendra à coïncider arec celui-ci, c'est-i-dire qu'il fera avec le premier plan de segmentation un angle dont la valeur se rapprochera de $90^{\circ}$.

Si l'on pourait, pendant la segmentation, reconnaitre toujours, par quelques caractères, le blastomère le plus avancé, on pourrait facilement déterminer, d'après sa position, la direction du plan de symétrie de l'embryon. Mais, comme cela n'est pas possible, lu moins dans les conditions actuelles de nos moyens de recherche, il s'ensuit que la détermination exacte de la direction de ce plan de symétrie ne peut être faite a l'avance. Nous devons lonc nous en tenir a cette conclusion que la direction du plan de symétrie du futur embryon peut osciller entre les directions du premier et du deuxième plan de segmentation de l'oeuf, et coincider parfois arec l'un ou avec l'autre de ces deux plans.

Le déplacement que les blastomères peuvent subir pendint la segmentation et, par conséquent, la place que le blastonère le plus avancé occupe dans l'agrégat cellulaire et que le blastomère de la phase limite occupera définitivenent, sont dépendants des conditions mécaniques dans lesquelles la segmentation a lieu. Or, ces conditions, ainsi que je l'ai démontré (1), sont, en glande partie, déterminées par l'état physiologique des autres cellules de l'angégat cellulaire: elles sont donc très complexes et en même temps elles peuvent varier dans les différents oeuf's d'une même espèce. Il s'en. suivra que, dans les embryons d'une même espèce et issus des geufs provenant d'un mème individu, lorientation du plan de symétrie par rapport an premier plan de segmentation ne sera pas la mème, mais pourra subir toutes les oscillations possibles entre les limites que nous venons d'établir.

Il sulît d'examiner les travaux publiés jusqu'ici sur cet

(1) Giglio-Tos E. - Les Problemes de la Fie. 1' Partie, p. 227. 
interessant sujet, pour nous conraincre que ces conclusions theroriques coincident parfaitement avec les résultats de l'expérience. On sait en eflet que le plan de symétrie de l'embryon ne coincide pas toujours avec le premier plan de segmentation, mais que, alu contraire, il a bien sourent une direction intermédiaire entre le prenier et le deuxième plan de segrnentation, dans les cats mémes do développenent parfaitement normal. C"est ce que les tramax de liopscir (1), et de Crapp (2) ont démontré très clairement.

Lorigine de la șrmétrie bilatérale peut donc itre expliquée parfaitement sans recourir i l'hypothèse d'une constitution quelconque préexistant dans l'oeuf. Mon interprétation de l'ontugénèse est, à ce point de rue, beancoup plus satisfaisante; car elle nous permet d'expliquer exactement non seulement les cas de coincidence entre le plan de symétrie de lembryon et les deux premiers plans de segmentation, mais aussi les cas dans lesquels le plan de symétrie a une direction intemérliaire entre ceux-ci. Ces derniers cas, au contraire, ne peurent receroir aucune explication si l'on suppose, ainsi que le font plusieur's hiologistes, une relation étroite entre la constitution de l'oeuf et l'embryon futur, et que le premier plan de division partage l'veut en deux moitiés correspondant aux moitiés gauche et droite de l'embryon.

Les conclusions que nous venons d'établir pour la direction du plan de symétrie de l'embryon dans le cas de symétrie hilatérale, peurent aussi s’étendre aux animaux à symétrie rayonnée.

Vous arons ru, en ellet, que lil symétrie rayonnée ne levient telle qu'au fur et it mestre que le déreloppement progresse, mais quefrectivement, an commencement de sa formation,

(1) Kopscir F. - Ueber das Ferhältniss der embryonalen Achsen zu den dici orsten Furchungsebenen beim Frosch, in: Intern. Monatschr. Anat. Phys., XVII Bd. 1900, pp. 1-26.

(2) Chapp C. M. - Some proints in the Development of the Toud-Fish (Batrachus tane), in: Journ, of Morphol., rol. V, 1891, pp. 494-501. 
elle se présente comme une srmétrie bilatérale. Nous sarons, par exemple, que l'apparition des tentacules dans plusieurs Coelentẻrés n’est pas un phénonène qui s'accomplisie tout d'un comp arec une strnétrie rayonnée, nais qu'il débute au contraire arec une sumétrio bilatérale assez éridente. Le prenier tentacule fait son apparition quelque temps arant les autres, et est suivi par la formation du deuxième tentacule du coté opposé au premier, suivant une ligne qui peut itre considérie comme l’axe de sinnétrie de l'embryon. Les autres tentacules naissent par couple d'un cité et de l'autre de cette ligne, qui, par conséquent, se comporte parfaitement conme un axe de șrmetrie bilatérale. On sait même que, dans plusieur's cas, cette symétrie bilatérale originaire est encore reconnaissable lorsque l’animal possèle déjit parfaitement léveloppée la disposition rayonnée caractéristique de ses organes.

Or, suivant le raisonnement que nous venons de faire pour la symétrie bilatérale, nous pourons établir que, lans les animanx ì synétrie rạomée, le plan de leur symétrie bilatérale originaire aura une direction internédiaire entre le premier et le deuxième plan de segmentation, ou bien coincidant arec l'un ou l'autre de ces deux plans.

Je ne puis dire si les faits réels correspondent parfaitement is ces conclusions thérniques; car je ne connais pas de travaux qui, faits dans ce but, aient tenu compte le l'orientation de l'embryon des animaux rayonnés par rapport au premier plan de segmentation; mais j'espère que des recherches convenables, a ce point de rue, ne tarleront pas a apporter quelque éclaircissement dans cette importante question.

Il me reste maintenant it considérer les morlifications morphologiques accompagnant les phinomènes de prolifération cellulaire jusqu'ici considirés. Mais, je le répete, comme ces morlifications sont dépendantes des conditions mécanirques daus lesquelles elles s'accomplissent, nous ne pourons les déternine: sills une connaissance partaite de celles-ci. Cependant, 
si l'on s'en tient à la considération d'un cas très simple, si lon suppose, par exemple, que la segmentation de l'oeuf ait abouti a la formation d'une blastula typique, nous pourons nous convaincre facilement, d'après ce que je riens d'exposer au chipitre IX, que la formation de la deuxième lignée de cellules aux dépens du liquide de la cavité de segmentation entrainera la gastrulation par invagination, et cette invagination continuera pendant la formation des lignées interradiaires ou bilatérales.

Ians ce cas donc, la formation de la gastrula sera contemporaine arec l'apparition de la sýmetrie bilatérale, c'est-i-dire que cette sýmétrie, n'existant pas encore à l'état de blastula, se présentera dans l'agrégat cellulaire durant la gastrulation.

En résumé, nous conclurons:

$1^{\circ}$ L'origine de la symétrie bilatérale est dénendante de

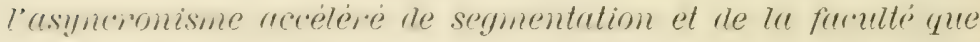

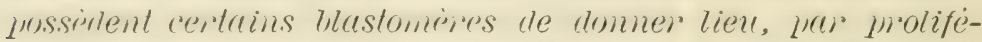
ration, à des lignées cellulaires spéciales, sous l'action de la mobiose des cellules de la deuxième lignée.

$2^{\circ}$ La symétrie du futur embryon est donc préexistante

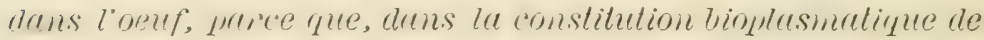
celni-io, resintent le rythme dle segrnentation et la nuture des blastomères qui en dériveront.

$3^{\circ}$ La symétrie est un phénomène purement mécanique

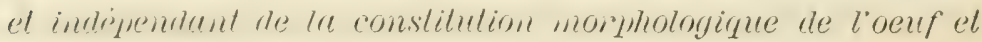
de la direction des premiers plans de segmentation.

$4^{\circ}$ La direction du plan de symétrie de l'embryon peut osciller, dans la même espèce, et dans les oeufs movenanl d'un même individu, entre les directions du premier et du

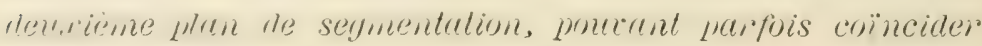
avec un de ceux-ci.

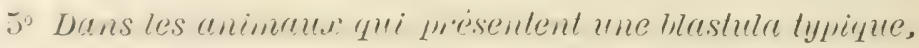
la symélrie bilaterale de l'embryon n'apparâ̂t qu'à la formation de la gastrula. 


\section{Chapitre XII.}

\section{Les phases ultérieures de l'ontogénèse.}

SOMMAIRE: Les principes de mon interpretation et lour examen - La symetrie de l'organisme et les symétries de ses parties - La complication progressive de l'embryon et de son milieu interne - L'automatisme des phénomènes ontogénétiques - L'organisme comme système symbiotique - Le fonctionnement des organes et la vitalité de l'organisme - L'équilibre symbiotique de l'organisme et ses conditions - Le renouvellement incessant de l'organisme - La mort - Les limites de l'existence de l'individu - Le rôle des stimalus physiques dans la détermination des différenciations - La localisation dans le temps et dans l'espace des différenciations histologique et morphologique.

Le lecteur qui a lu attentivenent les chapitres précédents aura pu connaitre parfaitenent les principes sur lesquels je base mon interprétation de l'ontogénèse.

Ces principes sont les deux suirants: $1^{0}$ ) le déreloppement monodique; $2^{\circ}$ ) la probiose des cellules.

Le déreloppement monolique est un des deux moles du déreloppement hétérorénétique, et nous arons ru, dans la I partie de ce trarail, que celui-ci est hasé sur un phénonene chimique très commun: la division d'une molécule en deux autres molécules inérales. Ce principe n'est done pas hypothetique: il est, au contraire, un des trois modes possibles et réels du developpenent bionoléculaire; il n’est pals basé sur une fropriété spéciale que j’attribue di la substance rirante, mais simplement sur une propriété chiniqute que l'on peut constater dans toutes les substances brutes, ru que, dans celles-ci, la dirision d'une molécule en deux autres inégales est un des phénomènes les plus communs.

Liautre principe, la probiose, est, lui aussi, un phénomène 
très frécuent daus la nature, dont on ne peut méconnaître l’importance, pour peu que l'on considère l'enchânement des fitres rivints et les rapports tress complexes existant entre eux au point de rue des moyens de leur existence. Je ne répeterai pars ici les exemples que j’ai déjil cités dans la I' partie do ce trarail, mais je ne hornerai a demander si de tels rapports ne peuvent pas exister entro les cellules mêmes d'un organisme. Un organisme pluricellulaire n'est-il pas un ensemble de cellules et, par conséquent, un ensemble d'organismes élémentaires vivant dans un milieu, le milieu interne, d'ou ils puisent leur nourrituro, précisénent comme l'ensemble des itres, le monde rivant, puise sil nourriture dans son milieu interne, lat nature? N'est-il pas, l'organisme, une symbiose très complexe de cellules, antant que le monde virant est une srmbiose des êtres? Pourquoi ne pourrons-nous pas supposer que les produits de sécrétion de certaines cellules de l'organisme servent à l'alimentation et au développement des autres? I) ailleurs, quoique les preures directes de ces rapports de nutrition entre les cellules d'un mème organisme ne soient pas très nombreuses, nous en possédons cependant quelquesunes dans les phénomenes de corruation des organes et dans l'inportance qu'ont certains organes, les glandes closes, par exemple, sur le développenent l'autres parties de l'être.

Je ne crois donc pas nécessaire de démontrer que ce deuxième principe de mon interprétation, la probiose, appartient, lui aussi, aussi bien que le prenier, al la catégorie des principes réels et naturels et qu'il n'est pas basé sur une hypothèse spéciale. Jespère que les Biologistes ne roudront pas nier l'existence et l'importance de la probiose dans les phénomènes de l'ontogénèse.

Cer leux principes étant admis, le développement ontogénétique se réduit, en lernière analyso, à une série ininterrompue et plus ou moins longue de proliferations cellulaires, c'est-ibrlire de lignées do cellules qui so succèdent dans un ordre déterminé. Chaque lignée a son origine dans une cel- 
Iule d'une lignée précédente et dans la nature dos substances chimiques constituant le milieu interne.

Le point de départ pour la formation des omganes résultant de ces lignées cellulaires est donc originairement une cellule seule; mais comme, dans le déreloppenent monolingue, l’apparition de cette cellule est suirie de l'apparition d'autres cellules qui arrivent a leur tour à la phase de la première, il s'ensuit que, dans l'etude du déreloppement des organes, il nous semblera parfois que ceux-ci tirent leur oririne non d'une seule, mais de plusieur's cellules. Cependant les recherches minutieuses embryologiques qui ont été faites jusqu' ici plailent moins en faveur de l'origine des organes d'un groupe de plusieurs cellules que d'une cellule unique. Lorsqu'on suit attentirement et rigoureusement la formation d'un organe dès sa première origine, on troure constamment que le point de départ originaire est une cellule seule. Or, cette constatation est en parfait accord arec le résultat théorique auquel nous sommes arrirés en partant de notre interprétation de l'ontogénèse.

On comprend d'ailleurs facilement que chaque prolifération cellulaire, chaque lignée de cellules de nouvello formation, toujours suivant le déreloppement monodique, donne lieu à la présence, dans l'agrégat cellulaire, d'un certain nombre de cellules dont la nature peut itre le point le départ pour une nourelle prolifération, et, par conséquent, pour une érolution difrérente et pour une différenciation ultérieure. Mais en nuème temps, la probiose de ces cellules pent, par les produits de sécrétion de celles-ci, servir à la prorluction des nourelles lignées cellulaires.

Or, si toutes ces lignées cellulaires, quel qu'en soit le nombre, se produisent suirant le développement monodique, ainsi que je le suppose, il en dérive nécessairement que nous derons appliquer à chacune d'elles les déductions et les conclusions possibles que nous renons d'appliquer à la première lignée. Dans chacune de ces lignées, il y aura donc une cel- 
lule qui sera la plus arancẻe dans l'érolution caractéristique de la lignée et d'autres qui se trouveront dans des pluases intermédiaires. Inus chacune d'elles aussi, le rythme de segmentation pourra être ralenti ou accéléré et, par conséquent, il donnera origne it une simple polarité, ou bien à une symétrie rayonnée, ou bien encore à une symétrie bilatérale, pourvu, cela est bien entendu, que se vérifient les conditions nécessaires, telles que je riens de les énoncer aux chapitres précédents.

Dans chaque groupe cellulaire formé par ces lignées de cellules, et, par conséquent, daus chaque formation morphologique ì laquelle ces groupes peuvent donner lieu, c'est-ii-dire, cn d'autres termes, dans chaque organe de l'embryon, nous jourrons constater une simple polarité, ou bien une symétrie rayonnée, ou bien encore une symétrie bilatérale, et cela, indépendamment de la symétrie de l'embryon, considéré dans son ensemble. Mais comme les parties de l'embryon peurent, it leur tour, présenter des parties secondaires et celles-ci des parties tertiaires etc., il s'ensuit que chacune de ces parties présentera une symétrie spéciale. P'ar conséquent, on pourra distinguer dans les or granismes des symétries d'ordre diflérent: une șrmétrie de premier ordre pour l'organisme considéré dans son ensemble; des symétries de deuxième, de troisième ordre etc. pour les parties secondaires, tertiaires, etc. de l'organisme même.

Dans l'immense variété que nous présente la nature, nous pourons trouver des exemples de ces dirrérentes sortes de symétries et de leurs combinaisons. J'en citerai seulement quelques-uns.

Ians plusieurs siphonophores, la colonie, c'est-it-dire l'ensemble de l'organisme issu de l'oeuf, ne présente pas une véritable symétrie, mais seulement une polarité, dans ce sens que les parties ne sont pas disposfes suivant une symétrie bilatérale ou rayonnée, mais originairement suivant une ligne unipue le long du stolon. Cependant, ces parties présentent 
une symétrie distinctement rarionnée. Nous arons donc ici un exemple de simple polarité de premier ordre combinée avec une șrmétrie rayonnée de deuxième ordre. I'autre part, certaines parties de leur corps, telles que les boucliers et les boutons urticants, présentent une symétrie bilatérale.

Dans d'autres Siphonophores, au contraire, dans les Disconanthes, par exemple, la symétrie de l'organisme est rayonnée ainsi que la symétrie de ses parties.

Dans la plupart des Coelentérés, la strmétrie rayonnée de premier ordre de leur corps est combinée arec la symétrie bilatérale de deuxième ordre des loges ou des cloisons ou bien encore des tentacules.

Dans les Vertébrés, Ia symétrie de premier ordre de leur corps est bilatérale; mais les parties de celui-ci possèdent ¿ leur tour ou bien une stmétrie bilatérale très distincte, ou bien encore une simple polarité. Le tube nerveux, par exemple, présente une synétrie bilatérale, qui se rérèle bien distinctement pendant son dérelopnement. Au contraire, les segments primoldiaux ne manifestent, dans leur développement ultérieur, qu'une simple polarité.

Je cite ces exemples pour fitire remarquer que la strmétrie des organismes n'est pas aussi simple qu'on lit considère généralement, et qu'elle peut résulter d'une combinaison parfois très complexe de strmétrie:s d'ordres diflérents. Or, toutes ces combinaisons peurent ètre expliquées très facilenent par l'interprétation de l'ontogénèse suivant le déreloppement monodique. On troure dans celui-ci toutes les conditions nécessaires pour l'explication de tous les cas possibles, même les plus complexes.

A mesure que le développenent progrresse, la constitution de l'embryon deviendra éridemment de plus en plus compliquée: car la formation de nouvelles lignées cellulaires provorquera une hétérogrénéité toujour's plus grande dans l'agrégat cellulaire représentant l'organisme. Tandis que, pendant la segmentation de l'osuf, l'agrégat cellularire ne résultera constitué que 
l'un certain nombre de cellules dans une des phases de l'érolution de l'oeuf, ainsi que je l'ai démontré, it la deuxième thase de l'ontogrinese, cette constitution sera plus complexe a cause do la formation de la deuxieme lignée cellulaire, et la complication s'accroitra arec la formation des autres lignées suivantes.

Il est riai que, à mesure que de nouvelles lignées cellulaires se forment, les cellules de lignées précédentes disparaissent peu i peu, en partie au moins, ou totalement, parce qu'elles arrivent graduellement à la phase qui est le point de lépart pour une nouvelle érolution; mais cette disparition, yui tendrait ì simplifier la constitution de l'organisme, ne sera pas suffisante pour compenser la complication qui va toujours croissant. Ainsi que nous l'avons ru, d'une seule lignée cellulaire peuvent dériver non seulement une, mais deux ou plusieur's autres lignées; par conséquent, la disparition d'une seule de celles-ci peut etre suivio par la formation de deux ou plusieurs lignées.

Nous devons arriver à des conclusions analogues pour le milieu interne.

Le milieu interne de l'oeuf, formé par ses substances deutoplasmatiques, est relativement assez simple; mais le milieu interne de l'agrégat de segmentation, constitué par les produits de sécrétion des cellules aux différentes phases de l'érolution de l'oeuf, doit itre inévitablement plus complexe. De mème, le nilieu interne de l'agrégat cellulaire, à la deuxième phase do l'ontogénèse, sera nécessatrement encore plus compliqué: car il contiendra, en partie au moins, les produits de sécrétion des cellules de segmentation et des cellules de la deuxième línée. Ie cette manière, le milien interne de l'embryon, lorsque les lignées cellulaires qui le constituent seront nombreuses, atteindra une constitution d'une très grande complexité.

Il est vrai, ici encore, rue la disparition de certaines substances chiniques alssorbées par les cellules qui s'en nour- 
rissent tendra à simplifier la constitution du milieu interne; mais, dans ce cas encore, ce phénomene ne pourra compenser la complication toujours croissante.

La faculté quiont les organismes d'arrivel' graduellement it une constitution très complexe en frartant d'une constitution heaucoup plus simple, cette fuculté, qui est une des manifestattions merreilleusos de la rie, n'est pas due it une furce spéciale du développenent, mais tout simplenent an morle mème du développement. Chaupe phase de l'ontoninese est la cause de la phase suirante, c'est-i-lire qu'elle prépare tuntes les conditions nécessaires pour l'accomplissement de l'autre phase.

Ce phénomène de préparation graduelle du milien interne apte au déreloppenent des phases successives peut arriver, dans quelques cas, jusqu'a la préparation non seulement des substances chimiques nécessiares à la nutrition, mais aussi le certaines conditions physiques indispensables pour l'accomplissement des phénomènes chiniques de lat vie. Nous en arons des exemples frappants et démonstratifs dans les animaux autothermes.

I)ans ces aninaux, les réactions chimiques nécessaires à l'entretien de la vie exirent, comme nous le savons, un certain degré de température. Or, tant que lorganisme se trouve dins les phases de son développement embryonal, les réactions chimiques s'accomplissant dans son nilieu interne, particulièrement lor's des premier's moments de l'ontogénèse, ne sont lats suffisantes, soit par leur petit nonbre, soit par leur natture, it développer la quantité de chaleur nécessaire à l'accomplissement des réations successires. Cette quantité de chaleur doit donc itre fournie jar le milien externe: dans les mamnifères, par le corps de la mère. Mais, à mesure que le déreloppenent progresse, les réactions chiniques, derenant plus nombreuses et plus complexes à cause de l'hétérogénéité toujour's croissante de lorinasme, peurent itre suflisantes at produire par elles-mèmes la quantité de chaleur nécessaire i l'entretien des phénomènes de la rie. 
On peut donc dire, en peu de mots, que l'organisme se perfectionne et se complique par lui-mème, pendant son déreloppement ontogénétique, en nous présentant, dans ce graduel autoperfectionnement, un exemple de cet automatisme qui nous frappe tant dans l'itude des phénomènes biologiques. Mais nous venons de roir dans ce cas que cet automatisme n'est pats la manifestation d'une force ou d'une propriété spéciale de la substance virante, mais tout simplement une conséquence naturelle du mode d'accomplissement des phénomènes vitaux.

Revenons maintenant à l'examen de l'organisme cellulaire considéré comme un système symbiotique de cellules, et nous en tirerons des conséquences qui, je l'espère, ne seront pas sans intérêt dans l'étude des phénomènes vitaux.

Tout système șmbiotique est constitué, comme nous le sarons, par trois facteurs: par le milieu ambiant, par la qualité des membres, ou unités constituantes, et, dans une certaine mesure, par le nombre de ces unités. Le fonctionnement du système symbiotique ne peut donc se faire qu'en tant que ces trois ficteurs existent et que les rapports entre eux sont cenx que leur nature exige. C'est dire que si l'un de ces facteurs subit un changement, les autres aussi doivent se modifier corrélativement, sans quoi le șistème symbiotique ne peut plus fonctionner.

Or, dans l'organisme, ces trois facteurs sont représentés par les liquides, sang, lymphe ou autre substance, dans lesquels baignent les cellules; par les cellules, dont le nombre est juscu'i un certain point déterminé pour chaque phase du dérelopjement et dont l'ensemble constitue les organes ou les tissus. (que ces cellules puisent leur nourriture dans le milieu interne, nous n'en pourons douter; qu'elles s'entr'aident réciprofuement dans l'accomplissement de leurs phénomènes vitaux en sécrétant des substances pourant servir d'alimentation aux autres, c'est ce que nous pourons arguer indirecteinent de l'étude des phénomènes de corrélation et directement 
de l'action physiologique de certains produits de l'organisme sur les autres organes.

La vie de l'orranisme considélé dans son ensemble est Ionc le résultat de la vie des cellules qui le composent; et si celles-ci virent, c'est que les conditions nécessaires au fonctionnement du sýstèmo symbiotique se trourent réalisées dans l'or ganisme mème. Tant que ces conditions existeront, l'organisme rirra; mais il périra inévitablement dès que celles-ci manqueront.

$\mathrm{Ol}^{\circ}$, le résultat de la rie des cellules constituant les organes est la rie de ces orianes mèmes: c'est ce que nous appelons le fonctionnement de l'organe.

La sécrétion des différentes substances de lat part les organes, par exemple, des sucs gastrique, entirique, pancréatique etc. par des cellules de l'estomac, de l'intestin, du pancléas etc. est une conséquence inéritable et directe de la vie de ces cellules, c'est-it-dire des reactions chimiques particulières caractérisant l'accomplissement de leurs phénomènes vitaux. L'absorption de certaines substances du sang par les cellules réuales et l'émission d'autres substances catractérisant l'urine ne sont que les manifestations des phénomènes chimiques d'où résulte la vie de ces cellules.

Dans ma conception, les fonctions des organes n'ont pas pour but la vie de l'organisme. Elles se font, au contraire, d'une manière absolument indépendante de ce but final; et si leur accomplisement aboutit a ce resultat merveilleux qui est la vie de l'etre, cela n'est qu'une conséquence nécessaire de la nature même de ces fonctions et des relations existant entre elles; relations desquelles dépend le fonctionnement de tout le système șmbiotique constituant l'ètre et, par conséquent, la ritalité de l'organisme. En peu de mots, l'existence et la fonction des organes n'ont pas un but, mais une cause; et la vie de l'organisme n'est pas leur but, mais tout simplement leur conséquence naturelle.

Certes, si l'on considère l'organisme pluricellulaire lorsqu'il 
a atteint son complet déreloppement et, par suite, au moment de sia plus grande complexité, il nous parait presque impossible qu'une constitution si compliquée et si merveilleuse se soit formée sans un but déterminé.

L'ensemble d'organes si diflérents et pourtant convergeant par leurs fonctions multiples it un efret unique, la vie de l'organisme; cet éfuilibre merveilleux dans le fonctionnement de tant de parties nous rend presque impossible la conception de l'organisme sans l'idie d'une finalite, et notre esprit se laisse facilement entrainer par les principes peu scientifiques de la tileologie. Mais je crois que ces difficultés disparaissent, en grande partie du moins, si nous considérons le mode de déreloppement de l'organisme.

J'ai démontré que, à cause du développement monorlique et de la probiose des cellules, l'ensemble de l'organisme va se transformant sans cesse par la formation de nouvelles lignees cellulaires. Il s'ensuit que tout organisme, considéré dans deux moments successifs de sa vie, ne peut jamais èire parfaitement égal à lui-mème. Mais ce changement continu dans les parties constituantes entraîne nécessairement, et nous l'arons vu, un changement dans le milieu interne, à cause des nouvelles substances chimiques que les cellules rersent dans celui-ci. I.e système symbiotique change donc a chaque instant; et si l'équilibre de ce systeme se maintient, si, par suite, l'organisme tout entier est vital, c'est que les relations entre les fircteurs du systeme symbiotique sont toujours telles que ces facteurs l'exigent.

On comprend donc facilement que le développement progressif de l'organisme n'est possible qu'en tant que ces conđitims d'équilihre existent; mais si elles font défaut, l'équilibre (xit lompu, le direloppement s'arrête et l'organisme périt.

supposons, par exemple, que pour une cause quelconque le milien interne ne soit pas parfaitement tel que, à un instant donné du développement, l'exige la prolifération de certaines cellules. Eridemment, celle-ci pourra subip ou hien de simples 
perturbations ou bien encore l'arrêt même. Cela dépendra naturellement de l'intensité de la morlification du milieu interme. Mais il est évident que l'organe dont la formation dépend de la prolifération susdite ou bien subira des déformations plus ou moins grandes, ou bien encore ne se formeria pas du tout.

Cette perturbation dans le déreloppement normal pourra par elle-meme itre sullisante prur rompre l'équilibre strmbiotique et le rendre impossible. Le développenent s'arrètera donc dès ce moment et l'oréanisme périra. Mais si elle n'est fas trop forte, le srstème srmbiotique, quoique altéré dans ses conditions, pourra néanmoins se maintenir et le réveloppement progressera.

Il faut cependant remarquer que, dans ce cas, l'anomalie dans la formation de la lignée cellulaire susdite pourra amener une altération plus grande dans le milieu interne; car nous savons que la nature de celui-ci dépend de la nature des cellules constituant l'organisme. Il pourra donc arriver que les causes de perturbation de l'équilibre symbiotique, insullisantes aupararant pour le ronpre complètement, saccroissent de plus en plus a mesure que le déreloppenent progresse, et acquièrent une intensité telle que la srmbiose et, par suite, la vitalité de l'organisme ne soit plus possible. Le phénomène de la mort se produira alors quelque temps apres la première altération du milieu interne, mais la cause frimitive n'en serat pas moins à celle-ci.

Tons arrirons encore it des conclusions analognes, si, an lien de supposer une altération dans l'un des facteurs du stistime symbiotique, dans le milieu interne, nous la supposons dans l'autre, dans la nature des cellules. Cirr, si l'on suppose, parr exemple, que, jour une cause quelconque, l'érolution d'une lignée cellulaire ne se fasse pas normalement, on bien cette lignée n’arrivera pas à donner liou a une cellule qui puisise itre le point de départ pour une autre lignée, et alors le diveloppenent s'arretera dans ce point et, peut-être, l'équilibre 
étant rompu, la mort s'ensuirra immédiatement; ou bien encore la constitution do cette cellule ne sera pas parfintement telle que les conditions du développement normal l'exigent, et alor's le systime symbiotique en subira des perturbations qui pourront le condinire plus ou moins tard a la mort.

Je crois que des cas semblables à ceux que je viens de considirer it un point de rue thérique, ne sont pas rares dans lat nature. Frappés de la perfection des organismes virants, nous sommes portés à roir daus l'ontogénèse un phénomène merveilleux par sa régularité et par la précision de son arrirée au but final, et nous négligeons les cas, assez nombreux, dans lesquels ce but n'est pas atteint et ou le développenent est interrompu plus ou moins tard. Je fais allusion à ces cas d'arortement yue nous pourons constater chez tous les animaux, et dont la cause ne peut résider que dans un développement imparfait. Mon interprétation le l'ontogénèse et la conception de l'organisme comme symbiose, nous permettent d'expliquer ces cas d'une manière rationnelle et scientifique.

I)'ailleurs, comme les facteur's du sýstème symbiotique, le milieu interne et les cellules, sont, dans l'ontogénèse, reliés entre eux par des rapports trés étroits et réciproques; comme la constitution du nilieu interne dépend de la nature des cellules qui l'ont produit, et que la nature de celles-ci dépend, en partie au moins, de la constitution du milieu interne au moment de leur formation, ainsi que nous l'arons ru, il s'ensuit que l'un de ces facteur's ne peut rarier salls prorofuer une Tariation dans l'atutre; mais il s'ensuit encore, en mème temps, que si l'un est normal, l'autre aura une très crrande prohabilité de l'itre, lui aussi. Et comme l'origine primitive du milieu intrune et des lignées cellulaires est l'oenf, duquel, nous le sarnns, dirrivent toutes les lignées de cellules constituant l'emhryon, il est évilent que, supposé normale la constitution de loouf et farorables les conditions dans lesquelles celui-ci derra se développer, il n'y a pas de raisons pour arlmettre yne le dereloppenent ne se poursuive pas nomalement. 
La condition essentielle pour le hiveloppement normal de l'organisme réside donc dans la constitution de l'oeuf, les agents extérieur's n’étant que les comlitions accessoires farorables a ce phénomène. Nous renrons d'ailleurs, dans une autre partie de ce trarail, comment cette constitution normale de l'oeuf peut être atteinte dans les organismes.

I)ans les considérations que nous venons de faire, j'ai toujours: distingué la période du développement ontorénétique des autres périodes de l'existence des organismes. Mais il est èvident que c'est lia une distinction tout-it-fitit scolastique et que je ne l'ai suivie que pour plus de commolité. I'ailleurs, cette distinction n'a pas de raison d'ètre au point de vue scientifique; car les phénomènes caractérisant l'ontogénèse ne sont pas, en dernière analyse, différents des autres phénomènes qui s’accomplissent pendant la vie de l'organisme juśru'a sa mort; et d'autre part, une distinction nette entre les périndes rle la vie des animaux n'est pas toujours possible, ru que les passages de l'une a l'autre s'accomplissent d'une manière gratuelle et insensible.

Lassertion du sarant Biologiste Driescn que " toutes les manifestions morpholoriques qu'un organisme présente jusqu'a sa mort doivent ptre comprises daus le domaine du déreloppement "est, selon moi, parfititement exacte, d'autant plus si l'on comprend le mot "morphologirque dans sa plus large signification.

Je dis a dessein «dans sa plus large signification» parce que les phénomènes que les orominisnes subissent pentant leur vie ne se révelent pas toujour's patr des changements de forme dans leurs parties, ou du moins, ces changements ne sont pas toujours apercerables par nos moyens d'observation.

Certes, la périorle dans lamuelle s'icemplissent les manston. mations organiques les plus frappantes est, sans contredit, la périorle entryonnare et la raison en est éridente. Inans celle-ci en effet, il y a une véritable formatisn l'organes, accomparnese d'une prolifération cellulaire très active qui accroit le nombre 
des cellules à mesure que le déreloppement progresse. Ces phénomènes sont dune hien visihles ef les changements de forme incontestahles. Mais les que les organes ont acquis le héreloppenent que les conditions du systeme symbiotique de lorganisme comportent, l'accoissement n'a plus lieu, et les changements de forme yu'ils subissent ultérieurement peurent itre tellement atténués qu'ils échappent presque completement a notre observation. Iess lors, faut-il amettre que les organes ne subissent plus le champements? Faut-il conclure que les béments constitumt les oryanes sont toujours les memes pendant toute la vie ultérieure de l'organisme? Ce serait lit une conclusion erronée.

Nous sarons, par l'étude des épithéliuns, des productions épithéliales, poils, ongles, etc, du sang, et de plusieurs autres tissus, que les éléments qui les constituent se renouvellent sans cesse pendant toute lat vie, et je suis parfatement convaincu qu'un phénomène semblable le renouvellement incessant ne tardera pas à ètre constaté dans les éléments des autres orwanes.

Tout organisme donc, alor's mème qu'il nous parait immuable, est, au contraire, le siege de mutations continues. Mais, comme les éléments noureaux oceupent la place de ceux qui ont disparu, ce renourellement peut aroir lieu sans entrainer des changenents de forme bien évidents, precisément comme le renourellement des grouttes, dans une chute d'aan, ne change pas la forme de celle-ci.

Mais, tout en admettant que ce renouvellement existe, une antre ruestion se présente i notre esprit. Sont-elles, ces cellules qui succèlent aux autres préexistantes, parfaitement illentiques it celles-ci? Posserlent-elles une constitution chimirque hioplatsmatique précisement égale it celle qu'araient les cellules qu'elles substituent?

Vus morns l'investigations chimiques ne nous permettent fras dr réponder a cette question par des preuves directes et positives; mais, faute de mieux, nous pouvons recourir à des indices indirects, qui, je le crois, ne sont pas moins sûrs. 
J'ai déjà en l'oecasion de faire remilruner, au chapitre II, que les caracteres d'oldre histologigue ne sont pas suflisants pour nous renseigner sur la véritahle constitution hioplasmatique des cellules, vu que des iléments de la mène nature histolorifues peuvent présenter des caracteres dordre chimirque hien différents. J'ai cité alor's des exmuples démontrant que, non seulemont dans les animaux d'especes diflérentes les élements histologirues de mème nature sont tres probablement différents an proint le vue chinique, mais que der diflèrences chiminues peut-itpe existent ausi entre les béments histologiquement égaux d’un mème animal dans les diverses parties de son corps ou dans les phases différentes de sa vie.

Or, cette différence entre les éléments histologiquement égaux d'un organisme dans les différentes phases de sa vie est une prenre érillente en fireur de l'inégalité chimique des éléments qui se substituent.

Nous pourons d'ailleurs en trouver d'autres exemples dans les manifestations les plus commmes de lat vio les organismes.

Les cellules de l'intestin des nouveau-nés ne sont pas, très probablement, ilentiques aux cellules intestinales le l'adulte: car si cette ilentits existait, on ne comprentrat pas l'impossibilité où sont les premières de digérer les substances que l'intestin de l'adulte digère très bien. Nous pouvons arriver a une conclusion analogue pour ce qui regarde les organes foie, pancréas, annexés à l'intestin.

On sait que les poils se renourellent sans cesse; mais il est bien facile de constater que les poils qui se substituent graduellement aux antres qui tombent, ne sont pas farfititement identiques à ces derniers. La différence entre eux est peut-

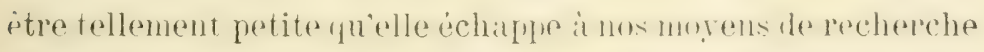
si nous examinons les poils dans deux phases successives de la vie; mais elle derient saisissable et manifeste, si l'on con-

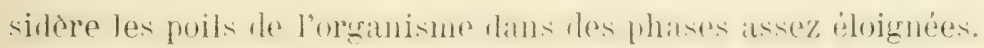
A une conclusion analogue nous arrivons par l'examen du renouvellement de la couche cornée de l'épiderme. 
Ces exemples, anxquels je pourrais encore en ajouter plusieurs autres tirés aussi hien les animaux que les végétaux, nous démontrent que, dans cet incessant renouvellement, l'organisme n'est jamais parfaitement éral a lui-mène dans deux differentes phases de sit vie. Mais je ne crois pas necessaire d’insister darantage sur ce lait, vu que les caractères mèmes yui mous permettent de juger de l'ige des ìtres sont précisément basés sur les propriétés diffirentes que les parties de leur corps présentent dans le cours de leur existence.

Tout organisme, ou, pour plus de précision, sa partie somatique, fallourt, dinss son existence, une courbe que l'on peut comparer à une parabole arant une le ses extrémités au commencement de sa vie, á l'oeuf, et l'autre à la fin de sa vie, à la mort. On roit que, l'après cette conception, la mort naturelle, ce phénomène si intéressant et si inexplicable, ne serait que le hout de la série des transformations caractérisant la vie les itres; et comme cette série est déterminée par la nature de ses transformations et, en derniere analyse, par l'oeuf qui en est le point le départ et la cause primitive, on comprend facilement comment le bout le cette série (et, par suite, la mort) est, lui aussi, déterminé par la constitution de l'oeuf, et comment, par conséquent, ce bout est divers dans les différentes especes et constant, lans certaines limites bien entendu, pour chaque espece.

L'interprétation des phénomènes ontogénétiques basée sur le déroloppement monolique, tel que je l'ai exposé aux chapitres précélents, non seulenent s'accorde parfaitement arec cette conception de la vie comme une série de transformations snccessives, mais nons amène directement et inévitablement it elle, en nous permettant en mène temps l'en domer une explication rationnelle et scientifique.

Vurs arons ru que, pendiunt la segmentation, l'veuf progresse grinlurlement dans son évolution caractéristique en en atteignant peu à peu les phases les plus élnignées, tandis qu’il en abandonne les plus prinitives. Ce phénomène, nous l'avons 
ru, est une conséquence directe du mole de drireloppenent monodique, que j'al posé comme base de l'nntogenèse. Les dirers blastomères peuvent itre representes comme des indivilus qui, tout en étant de constitutions dillérentes, se trouvent néanmoins en marche dans la même direction. Or, un phénomène analogue s'itcomplit et se poursuit dans toute l'érolution ontogénétique.

De même que nous arons marqué les diflérentes phases de l'érolution de l'oeuf par des lettres 1,7 , c etc. représentant la constitution bioplasmatique de chaque blastonère, nous pouvons alssi, pour mieux concréter nos ilées, marquer les diverses lignées cellulaires se produisant successivenent dans l'érolution on togrinétique, par des lettres $A, B, C, I$ etc., où ces lettres représentent l'ensemble de chaque lignée, caractérisé évilemment par la constitution bioplasmatique des celIules qui la forment.

Soit done A la première lignée collulaire, la lignée des blastomères derrivant de la segmentation de l'veuf. Il est évident qu'a mesure que la deuxième lignée cellulaire $B$ se formera, les blastomeres de la lignée $A$ abantonneront succesirement les phases de cette lignie pur passer lans une des phases de la lignée $B$, et ce phénnmène se poursuirra tant que les conditions le permettent.

Le même, les cellules de la lignée $B$, en donnant lieu, comme je l’ai expliqué, à d’autres cellules de la lignée $C$. abindonneront successivement les phases de lia lignée $l^{\prime}$ funr fatsser à les phases de la lignée $C$ : ćest-it-dire que les cellules de la lignée $B$ disparaîtront graduellement, it mesure que se forment les cellules de la lignée $C$, de mène que les blastomeres de la lignée 1 auront lisparu partiellement on totalenent par la formation des cellules de la lignée bi. Le mème phénomène se produira dans les cellules r. lors de lit tormattion de la lignée I, et pourra se continuer l'une maniere analogue, quel que soit le nombre des lignées que nous supposons constituer l'évolution ontogénétique. 
Or, il est evident que cette succession de lignées cellulatres ne peut se farre at l'infini. La proliferration cellulaire, comme nous le sirrons, est suburdomnée it l'assimilation, et celle-ci exige deux conditions essentielles: une constitution bioplasmatique apte it laccomplissement des phénomenes chimiques de l'assinilation, ef $u n$ milieu contenant les substances nourlissintes nécessatires à ces phénomènes. Tant que ces deux conditions existent, l'assimilation est possible et lat prolifération cellulaire aussi; mais des que l'une d'elles manque, lassimilation et, par suite, la prolifération ne pourront plus avoir lieu.

I es limites dans la formation des lignées cellulaires caractérisant l'érolution ontogénétique d'un organisme quelconque sont lone déterminées par la nature du milieu interne et par lil constitution des cellules mêmes constituant les lignées. Certes, ces limites, nous ne pouvons les connaître, ru que nos moyens dobservation sont absolument insuflisants pour ce but; mais nous pourons arguer qu'elles doirent exister assurement, et qu'elles doivent être diflérentes non seulement pur les diverses espèces doromismes, mais aussi pour les direr's indiridus d'une même espèce.

En effet, comme la constitution de chaque lignée cellulaire est dépendinte de la constitution de la lignnée précédente, il en suit éridemment que les constitutions de toutes les lignées dépentent de la nature de la première; et comme celle-ci dépend de la constitution de l'oeuf, toute la série des lignées dépend bill conséquent de la constitution de l'oenf. Or, j'espere dímontrer, dans une autre partie de ce travail, que la constitution chimique de l'oeuf ne peut itre identique dans les diver's individus d'une même espèce et, à plus forte raison,

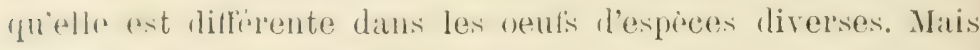
dès ce moment, il nous est facile de comprendre que l'interprétation de l'ontogénèse basie sur le développenent monorigue ferut mos "xpliquer parfaitment at scientifiquement comment lia nort est un phénomène inévitable et fatal, et 
comment l'existence des organismes a res limites, qui lui sont imposées par la constitution même lu corpse et par la nature des phénomènes biologiques.

Il nous reste à voir maintenant si untre interprétation est sullsante pour l'explication les phénomènes fondinentaux de l'ontogénesse, tels que je les ai énoncés dans le chapitre I de cette partie, à savoir: la prolifération cellulaire, les diflérenciations histologiques et morphologiques et la localisation des différenciations.

Je pourrais passer sous silence lit prolifération cellulaire, parce qu'on comprend facilement qu'elle n'est tout simplement que l'effet direct de l'assimilation, de lit part des cellules, des substances du milieu interne. Nais ce qui nous frappe plus vivement dans l'obserration du déreloppement ontogénétique, c'est que la prolifération cellulaire est d'une très grande irrégularité. La dirision des cellules ne se fait pas d'une manière régulière dans tout l'embryon et pendant toute la période de déreloppement; mais elle subit au contraire des oscillations. On constate, en effet, que les cellules en division sont plus fréquentes et plus abondantes dans certains points de l'embryon et a des phases determinées de l'ontogénèse.

Or, par mon interprétation de l'ontogrénèse, non seulement cet intéressant phenomène recoit une explication scientifique, mais il en devient même une conséquence tout-à-fiat naturelle. Puisque toute prolifération cellulaire exige deux conditions: la présence d'une cellule d'une constitution bioplasmatique apte it se nourrir aux lépens des substances spéciales du milieu interne, et la présence de ces substances dans le milieu interne même, quoi de plus naturel que la prolifération ne puisse aroir lieu qu'en des endroits détermines de l'embryon ou ces cellules apparaissent, et it des phases de la période de déreloppenent oi les substances spéciales se trouvent dans le milieu interne?

Mais c'est dans l'explication des diflerenciations histologiques et morphologiques et de leur localisation qu'on peut 
apprécier toute l'importance de mon interprétation, parce qu'elle nous permet d'expliquer d'une manière tres claire et fiacile ces phénomènes, qui sont, peut itre, les plus intéressants, les plus importants et, en même temps, les plus abstrus de l'ontogénèse.

Il fiut toujours se rappeler, dans cette étude, que les différenciations histologiques et morphologiques s'accomplissent dans des endroits détermine's de l'organisme et a des époques tixes du développement. Par conséquent, quelle que soit l'interprétation que l'on veuille donner de l'ontogénèse, elle ne pourra itre satisfaisante qu'à la condition de nous présenter une explication de ces phénomènes et en même temps de leur concomitance.

J'at démontré, dans les premier's chapitres de ce livre, qu'une différenciation histologique, quelle qu'elle soit, se réduit, en dernière analyse, à une différenciation chimique de la cellule: c'est-il-dire que celle-ci acquiert une constitution bioplasmatique bien déterminée, par laquelle elle sécrète des substances spéciales caractérisant précisément sa diflérenciation histologique.

Or, cette constitution chimique spéciale du bioplasma des cellules ne peut être acquise tout d'un coup. Le bioplasma des cellules ne peut $y$ arriver que peu it peu en passant par des phases intermédiaires.

Il est rrai que l'observation superficielle des phénomènes nous demontre, au contraire, que la diflerenciation histologique se lait presque d'un seul coup et lorsque la différenciation morphologique est accomplie, tandis que celle-ci se fait graduellement; mais c'est lid évidemment une simple apparence trompeuse, dont la cause en est at nos moyens d'investigation.

si nous royons que la différenciation morphologique d'un orratme s'acemplit peu ì peu, c'est que la formation graduelle de cet oryane se manifeste par un phénomene, l'atgrgmération de cellules, yue nous pouvons constater facilement par nos moyens de lecherhe. si nous constatons, au contraire, que la 
différenciation histologique d'une cellule se fait tout d'un coup, c'est simplement parce que les transformations successives que le bioplasma de cette cellule a subies dans toute la série des cellules, ses ancêtres, a échippé, completenent ou presque completement, ì nos observations.

Considérons, par exemple, l'histogénèse d'une fibre musculaire.

Comme cette fibre dérive, ainsi que tous les éléments de l'organisme, de la cellule oeuf, elle aura eu, elle aussi, une série plus ou moins longue de cellules, ses anceitres, constituant son arbre généalogique. Nais puisque la production de la substance contractile caractérisant la diflérenciation histologique de cette fibre ne peut avoir lieu que lorsque le bioplasma acquiert une constitution chimique roulue, il est érident que les cellules, ancêtres de la fibre, ne pouraient sécréter cette substance, faute de la condition indispensable pour sa production, c'est-i-dire de la constitution bioplasmatique.

Ce que nous appelons la rraie différenciation histologique de la fibre musculaire, caractérisée par la production de la substance contractile, se fitit lone dans cette fibre; mais celat n'exclut pas que les transformations nécessaires pour arriver a la constitution bioplismatique roulue se soient accomplies graduellement dans toute la série des cellules dont la fibre est issue. Il est même plus concevable et plus rraisemblable que ces transformations aient lieu et que la production de la substance contractile ne représente que la dernière phase de toute une période de préparation bioplasmatique aboutissant a la formation de la fibre musculaire.

La différenciation histologique ne serait donc que le résultat final d'une série plus ou moins longue de transformations chimiques du bioplasma cellulaire, et les divisions des cellules marqueraient les étapes de cette évolution rers un point déterminé. Nais comme la direction de cette évolution ost déterminée par la constitution des cellules et par la nature des substances chimiques du milieu interne, il s'ensuit que, 
l'apress cette interprétation, le stimulus qui agit sur les cellules pentant leur érolution et qui les dirige et les porte it leur dillérenciation caractéristique, est un stimulus de nature chimique.

Arec cela, je ne reux pas refuser absolument toute importance aux stimulus de nature physique; mais je crois qu'on doit les resierrer dans des limites plus restreintes que celles que plusieurs Biologistes leur accordent généralement.

Bien que je sois parfaitement disposé à admettre que les agents physiques puissent exercer une action sur la détermination de la diffërenciation histologique, je crois néanmoins que le résultat de cette action est toujours subordonné à la constitution chimique de la cellule qui la subit.

Considérons, par exemple, l'action de la lumière.

Nous sarons que la lumière, en agissant sur des cellules, peut provoquer la formation de pigments. Par conséquent, on dit d'habitude que cet agent physique produit des pigments. Mais c'est là, évidemment, une expression inexacte: car un examen plus attentif nous démontre que cette production est l'ellet de phénomènes chimiques proroqués dans la cellule par' la lumière, et que la cause réelle et efliciente en est ì la constitution chimique spéciale du bioplasma des cellules que nous arons considérées. Cela est si rrai que l'action de la lumiere ne donne pas les mêmes resultats dans toute sorte de cellules. La lumière n'est, dans ce cas, qu'une condition physique nécessaire ou bien utile pour l'accomplissement d'un phénomène chimique, de même que la chaleur l'est dans la plupart des reactions chimiques; mais le substratum rrai de ce phénomène réside, sans aucun doute, lans la constitution chimique des substances qui sont le siége des réactions.

lout en arlmettant donc que les agents physiques puissent joner un roile important dans la déternination des différenciations histologiques, je crois néammoins qu'ils ne représentent, dans ce phénomène, que les conditions nécessaires ou utiles pour son accomplissement, et que la cause fondamentale 
réside dans la constitution bioplasmatique des cellules et, par conséquent, dans les substances nourrissantes qui, en agris:ant comme stimulus chimigue, ont porté le hioplasma les cellules à une constitution déterminée.

Cette manière d'envisager le role des actions physiques dans la determination des différenciations me parait non seulement plus scientifique, mais plus exacte, et nous permet en même temps, en diminuant l'importance de ce rôle, de résoudre certaines questions qui ne pourraient receroir autrement une explication satisfaisante. Car, en subordomnant le rôle des actions physiques à la constitution chimique du bioplasma, nous donnons it celle-ci une importance capitale dans les phénomènes des diflẻrenciations histologiques; et, par conséquent, nous comprenons comment, dans certains cas, ces phénomènes peurent se passer de la réalisation les conditions physiques.

Il faut toujours se rappeler que, daus l'accomplissement des réactions chimiques, la cause efliciente réside dans la nature des substances réagissantes, ot que les actions phrsiques ne sont pas toujours des conditions indispensables, mais, parfois, seulement favorables i cet accomplissement. Très souvent encore, les actions physiques peurent se substituer l'une à l'autre et produire également le même résultat.

On sait que certaines réactions chimiques se font très rapidement at la lumiere du soleil, mais qu'elles ne s'accomplissent pas moins, quoique plus lentement, a la lumirre difluse. Pour d'autres réactions, il existe un degré optimum de température; mais entre certaines linites, c'est-it-dire au dessus d'un degré minimum et au dessous d'un degré maximun, ces réactions s'accomplissent égalenent. On sait encore que, dans d'autres can, à l'éléctricité, comme agent physique déterminant certains phénomènes chimiques, peut se substituer la lumière ou la chaleur.

Evidemment, la possibilité de l'accomplissement des réactions chimiques dans l'une ou dans l'autre de ces diffé- 
rentes conditions physiques est dépendinte de la nature des suhstances réagissantes; mais cos exemples, empruntés à la matière brute, nous démontrent que des relations analogues doivent exister entre les reactions de la substance vivante et les actions physiques agissant sur elle.

Ainsi, pour s'en tenir à l'exemple cité ci-dessus, tout en admettant que la lumiere peut proroquer dans certaines cellules la formation de pigments, nous pouvons néanmoins comprendre comment la production de cette substance est possible dans certains cas, à défaut même de cet argent physique.

Or, si lon admet que la diflérenciation histologique se fait peu it peu, par une préparation lente et graduelle, pendant laquelle les substances chimiques du milieu interne de l'organisme jouent le roile le plus important dans la détermination de la différenciation, on peut démontrer très facilement, d'après mon interprétation, comment la différenciation histologique s'accomplit dans un endroit déterminé de l'organisme et it des phases fixes du déreloppement.

Considérons, par exemple, une cellule, et supposons qu'une de ses descendantes, après être passée par les phases des lignées $A, B, C \ldots, N$ arrive a une phase $p^{n}$ de cette dernière lignée cellulaire, et qu'i cette phase, elle ait atteint une constitution bioplasmatique telle que sa diffẻrenciation histologique l'exige. Cette cellule, dès ces moment, acquerra les caractères propres de cette différenciation.

Mrais commes les cellules de la lignée $\mathrm{V}$ dérivent de cellules des lignnées précélentes.... $C$, et que les cellules de cette lignée $C$ lérivent de cellules de la lignée $B$, ainsi que celles de la lignée $I$, de cellules de la lignée A, il s'ensuirra naturellement que la position de la cellule $p^{n}$ dams l'arrégat cellulaire sera dépendante de la position des cellules de la lignée $N$, à laquelle elle appatient; que lat position de celle-ci dépendra de lat position les linnéess qui l'ont précédée, et ainsi de suite jusqu'à la lignée f, print de lépart les antres lignées. On roit donc

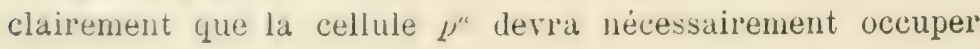


dans l'organisme une position hien literminee par la nature mème de sa differenciation histologique et par les phases qu'elle doit parcourir pour arriver all hout de son évolution.

Or, nous arons vu que cette érolution s"acemplit peu d peu par des transformations chimiques, dont les whases sont separées par des divisions cellulares, et que ces dirisions se succèdent d'une manière assez régulière, suivant un rọthme qui est le résultat des lurées des périodes assimilatrices des cellules. Il s'ensuivra, par conséquent, que la phase $p^{\text {in }}$ de la cellule susdite ne pourra etre atteinte quaprès un certain nombre de divisions cellulaires, nombre qui sera déterminé par le nombre même des phases par lesquelles lat cellule doit passer pour arriver it sa constitution definitive, et que, par suite, la différenciation histologique de la cellule $l^{\prime \prime}$ ne s'accomplira qu'après un certain laps de temps, à partir du commencement du développement.

Les différenciations histologiques et leurs localisations dans l'espace et dans le temps trouvent donc dans mon interprétation une explication suflisaute et scientifique. Par des raisonnements tout-a-fait analogues, on peut arriver a démontrer la localisation de la différenciation morphologique.

Si nous faisons abstraction de la différenciation histologique, nous pourons dire que tout organe n'est que l'agglomération de cellules. Or, cette agrglomeration est naturellement la conséquence directe de la prolitération cellulaire. On comprend donc facilement que la différenciation morphologique, résultat de lat prolifération cellulaire, doit, elle aussi, etre localisée dans des endroits déterminés de l'organisme et faire son apparition à des époques fixes du développement.

Malheureusement, nos connaisances sur les principes qui règlent la formation des organes et sur les causea déterminant leur forme caractéristique sont absolument insuffisantes: mais l'étude les phénomènes de l'ontogénèse nous démontre que, très souvent, ces causes et ces principes ne sont que d'ordre purement mécanique. 
Certes, les combitions micaniques dims lesquelles se troure un organe pentant sa formation sont, dams la plupart des cas, si complexes qu'il est prespue impossible de les apprécier à lemp juste valeur et, parfois meme, de les comnitre. La forme des cellules, leurs dinensions, leur's proprietés athésives plus ou moins accentuées, leur rythme meme de division, le roisinare diatres cellules ot les propriétés physiques ou chimiques de celles-ci otc. sont autant de causes qui peuvent influer sur la forme de l'organe en voie de développement; mais on pent ficilement se convaincre que toutes ces causes sont plus ou moins directement lépendantes de la constitution chimique des cellules. Nous avons done le droit de conclure que les causes mécaniques ou phrsinues déterminant la diflérenciation morphologipue sont, elles aussi, subordonnées it lit constitution chimique, et que celle-ci est, lans ce cas encore, la cause primitive et efficiente de la diflérenciation morphologique.

On roit done que, dans mon interpétation, la localisation des différenciations histologique et morphologique dans l'espace et dans le temps et la conconitance de ces différenciations sont les phénomènes qui découlent tout simplement des principes mèmes de l'interprétation. Lut, comme la vie extraenbryomnale de l'organisme n'est que la continuation du phénomène ontorénitique, atinsi que nous l'avons vu, on peut, maintenant, comprendre parfaitement pouryuoi et comment les caracteres somatiques font leur apparition dalls l'organisme à des époques déterminées de leur existence. 


\section{Chapitre XIII.}

\section{Les Problèmes de l’Ontogénése.}

SOMMAIRE: §I: Sur le développement de portions d'veuf (I Probléme) - § II : Sur le développement des blastomères isolés (II, III, IV Problémes) - \$ III: Sur le développement de grounes de blastomères (V P'roblème) - \& IV: Sur lo développement de portions de blastula (VI Problème) - $\$$ V: Sur le développement d'extraovats (VII Problème) - §VI: Sur le développement de blastomères imparfaitement isolés (VIII Problème) - §VII: Sur les résultats de la destruction de blastomères (IX, X, XI, XII Problèmes) - \& VIII : Des effets de la compression sur le développement ontogénétique (XIII, XIV Problèmes) - § IX: De l'action de la gravité sur le développement ontogénétique - $\$ \mathrm{X}$ : De l'action des agents physiques et chimiques sur le développement ontogénétique.

Nous avons ru dans les chapitres précédents que mon interprétation de l'ontogénèse, en se basant exclusirement sur les propretés de la matière et silns recourir à des hypotheses spéciales, peut nous donner une explication scientifique de tous les phénomènes caractéristiques et fondanentaux de l'ontogénèse, at savoir: de la sementation de loeuf, du rythme de segmentation, les phases de l'ontoginèse, des différentes sortes et combinaisons de symétries. de la prolifération cellulaire, des différenciations histologique et morphologique et de leur localisation dans le temps et dans l'espace.

Nous aroms donc les raisons plausibles pour croire que cette interpretation est exacte. Nais cela ne suflit pas encore. Il nous reste à démontrer que cette même interprétation, ses principes restant inmuables, n'est pas en desaccord avec les résultats de l'embryologie expérimentale.

Or, si non seulement ce désaccord n'existe pas, ce qui serait déjà suffisant pour la bonté de l'interprétation, mais si celle-ci 
nous donne encore une explication exacte et parfaite des résultats des expériences, les prenves en fitreur de son exactitule leviennent si nombreuses at acquibrent une telle importance que nous pourons esperer d'aroir interprété les phénomènes ontogénétiques d'une manière, du moins très voisine, de leur réalité.

Ians les pararraphes qui ront suive, je passerai en revue les diffërentes expérionces de l'embryologie expérimentale, expripiences que je présente ici comme des problèmes, dont la solution exacte doit dépendre des principes de mon interprétation, considérés comme des théorèmes.

$\$ I$.

Sur le développement de portions d'oeufs.

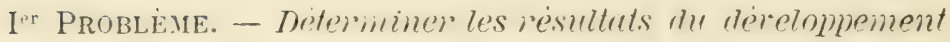
de portions d'oeufs excisées arant la segmentation.

Sorrtms. - Pour compreulre et léterminer les résultats le ce problème, il fiut arant tout aroir une idée absolument exacte de l'veuf et de ses parties, et surtout de ce qu'on entend géméralement sous la dénomination: cýtoplasme de l'ouf.

On indique d'habitude par ce mot tout le corps de l'veuf en dehors du noyau. Or, si nous exaninons attentivement la constitution re l'oeuf, nous pourons facilement nous convaincre que cette lénomination n'est pas parfaitement exacte.

Tout renf, quelle que soit sa petitesse, contient toujours des substancer deutoplasmatiques. Evidemment, lorsque celles-ci sont plus abondantes, ou bien encore lorsqu'elles sont accumulées dans une prartie de l'oeut, elles se rendent bien manifestes; tandis que si elles sont moins abondantes et uniforminent répandues dans tous le corps de l'venf, nous ne frowons guere les distinguer du bioplasmat ovulaire. C'est your cela qu'on dit généralement qu’il y a des oeuts sans 
deutoplasma, les oenfs alecithes: mits lo lentoplasma existe toujours, meme dius ces oeuls, quon anpellerait, par suite, plus exactement oeufs homolécithes.

Nons derous done distinguer dans l'vent deux substances: le bioplasma, substance vainent vivante et dans laquelle résile la propriété de l'assimilation, et le deutoplasma, substance brute, ne serrant qu'al l'assimilation du binplasma.

Or, si l'on examine lo morle de préparation le l'oeuf, on peut facilement se convaincre que la matse du bioplatinat. quel que soit le rolume total de l'oeuf, est toujours tres petite, parfois énomément petite, par raphort it la masse lu deutoplasma.

En efret, comme les cellules freminatives ot les oromonies menes, arant le commencenent de lit phase d'accroiscement, sont des cellules ne contenant pas encore de deutoplasma, elles sont tres petites, et leur rolune ne hipasse pats généralement le rolume de la plupart des autres ctullules de lorinaisme. Mais dès que la phase d'accroissement commence et que lorogonie subit sa hifërenciation caractéristique, le rolume saccroit. Cepenlant, cet accroissoment est plus le résultat de la production du deutoplasmal, sécrété frar le hioplasma de lorosonie, que d'une augmentation de la matice du bioplasma mème.

Il est rrai que celui-ci s'accroit pendant la phase l'accroissement; mais son rolume, au bout le cette phase, ne lépasse pas le double du volume qu'il avait aupararant. Or, le rolume total de l'oent est évilemment toujours do heancoup blus grand que le double du volume gu'il arait a la phate d'oromonite et l'excédent de ce volume est dû exclusivement à la masse du dentoplasma yui s'est accumulee dans l'oenf pendant la phase d'iaccroissement.

Or, dans les neufs, arant que lat formation du premier fustatu de sementation ait commence, la fetite masise lu binplatsma ovulaire se trouve accumulée dans une partie de l'oeuf, par exemple, an centre (oenfs alecithes ou centrolecithes), on bien encore à un les priles (oeuls télolécithes, et elle ne contracte 


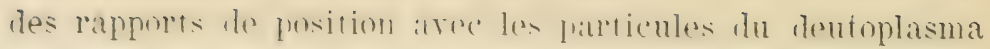
qu'alors seulement que le premier fuseau de sermentation se forme. Nous arons ru, dans la I Partie de ce travail,

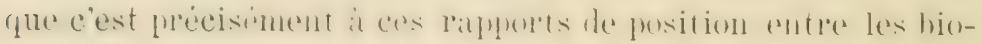
mores du hioplasma ef les farticules lu deutoplasmal gendant la cytodiérèse que l'on doit si, dans la segmentation, le deuwplasma est entrainé frasirement dins les ditrérents batstomères.

l'all conséquent, lor'sque, ditns les expériencess dootomie, on rxeise une portion l'nenf, si le premier fusean do division n'est pas encore formé, la portion excisée ne contient évidemment que de la substance deutoplasmatique.

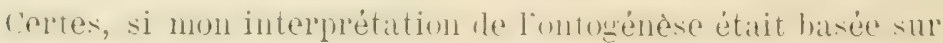
l'hypothèse de l'anisotropie de l'oeuf, je ne saurais comment expliquer le déreloppentent momal de celui-ci et lit formation dom embryon complet, apres l'excision doune portion du deutoplasma. Car il est très érident que si l'oeuf contenait dans son deutuplasma une distribution, quelle qu'elle suit, de praticules, ayant la moindre relation arec la structure du futur embryon, celui-ci derrait nécessitirement matnquer de quelques-unes de ses parties. Mais comme mon interprétation est basée sur l'isotropies las loeut, comme j'exclus absolument toute relation entere lit constitution morpholuginge de l'oente et lit structure de l'organisme qui dérive de sa segmentation, il est très

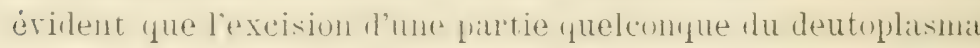

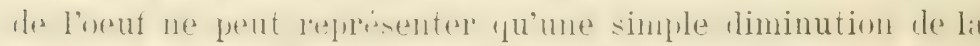
matse deutuplasmatique. (anels seront done les effets de l'excision de l'oeuf?

Le deutoplasinat, nou- le sitrons, n'est qu'me substance brute servant de nourriture au bioplasma de l'oeuf. Il est done indi-pensable pour son asimilation: at comme le dérloublement

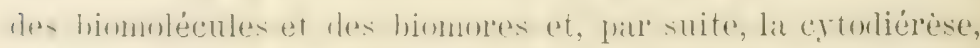
ne peut se faire sans l'assimilation préalable, il est clair que, si nous arrivions a soustraire à l'oeuf toute la masse deu-

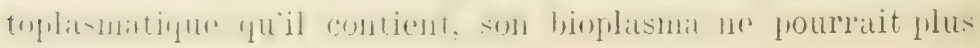




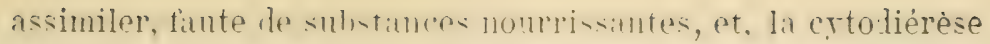
ne pourant s'accomplir, la sermentation n'aurait pas lieu.

Mais comme, dans les expériences d'ootomie, l'oeuf n'est

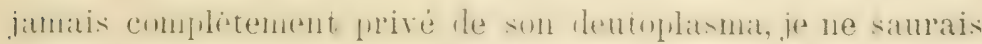

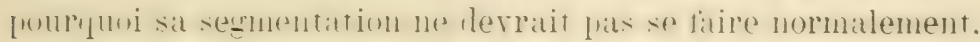

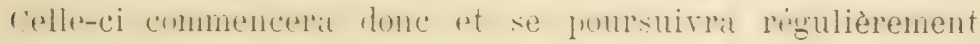
jusqu'à certaines limites.

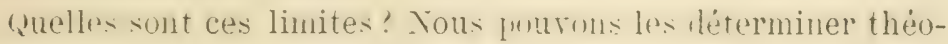
riquement d'une manière générale.

supposons, four nous en tenir toujours it lexemple cité anx chapitres précérlents, que las phates de lérolution le l'oenf " soient $b, c, d \ldots \mu$, et que celle-ci soit la phase limite de cette érolution. Cette phase ne purrat intre atteinte qu'après 7 di-

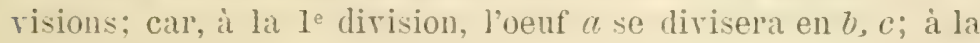
$2^{r}, c$ se diviserit en $d, e$; à la $3^{\mathrm{e}}$, e en $f, g$; a la $t$ e, $g$ en $h, i$;

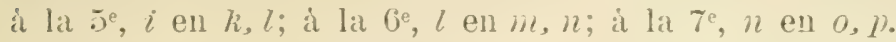

Or, chacune de ces divisions sera séparée de la suivante far une prérionle assintilatice, pentant latquelle le hioplasma

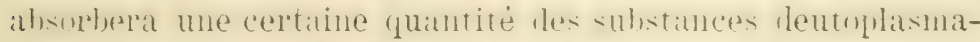
tiques. cet te quantitis représentera le minimun de deutoplasma imlispensible pour l'acconplisenente le levolution de l'oenf: car il est érident que si elle était inférieure à ce minimum,

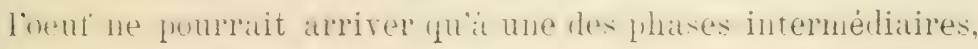
mais jumais à la phase limite.

si donc, dins les conditions normales, tous les oeufs ne

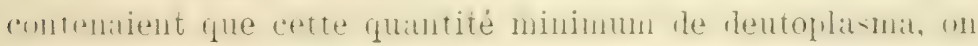
pourrait conclure que toute excision de l'oeuf, en diminuant

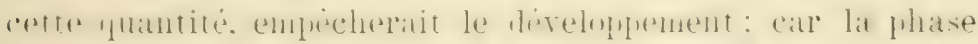
linite, puint de depart fump les antres evolutions, ne fourant ètre atteinte, le développement s'arrèterait. Dans ce cas, nous verrions la segmentation se poursuivre jusqu'a un certain point et puis s'arrêter.

Mais si l'on doit en juger d’après ce que nous constatons fré-

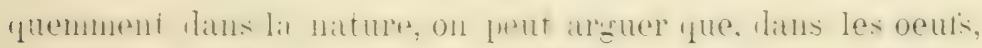
il y a frestue toujours une quantite le substances deutoplas- 
matigues de braturoup superieure a celle qui serait strictement nécessatipe. In eximen, mème superficiel, des phénomènes accomparnant le developpement des anmanx et des végétaux, wi, a l'achovement du déveluplement, dans les conditions normales, il y a un surplus de substances nourrissantes dont lorganisme $11^{\circ}$ a fias filit usige, nul demontre que lat nature, dans la préparation de ces substances, n'est pas du tout arare mais plutot très prodigue.

Or, cette surabondance de deutoplasma étant dunnée, on comprent fitcilement que, si, dans les expériences d'ootomie. lat partie excisée n'est pas trop gramle, le déreloppement de livent derrat se faire normalement et que l'embryon issu de celui-ci devra ètre complet et normal.

Mais si cette protie excisée est trop grande, c'est-it-dire si l'on sonstrait a l'oeuf une masse deutoplasmatique telle que ce yui reste le deutoplasma dans l'vent soit inférieur au minimum nécessaire pour l'achèrement de la série des phases caractérisant l'érolution de l'oeuf', il s'ensuirra évidemment que la phase limite de celle-ci ne pourra plus être atteinte et que le déreloppement de l'embryon sera nécessairement empêché.

Je dois, a ce propos, appeler l'attention des Biologistes sur certaines dérluctions et sur quelques expressions qui ne me paraissent pas parfaitement exactes.

Lal plupart des Biologistes qui ont étulié les résultats de ces intéressantes expériences d'ootomie, ayant constaté que la segmentation s'accomplit quand même, quel que soit le rolume le lit fratie le l'oent excisée, en ont conclu qu'alors mine que cette partie as tres grande, dans certaines limites hien entendu, le gurvor de léveloppement de l'veuf n'est pas amoindri. Mais l'exactitude de cette conclusion est tout-à-fait -nbrimuér it ce quon entend par pouroir de développement de l'oeuf.

Car si, par cette expression, on reut indiquer le pouroir inhérent it loeuf de lonnes origine à un embryon, la con- 
clusion ne me parait pas parfaitement exacte. Si l'on roit que l'oeuf, bien que privé d'une partie de son deutoplasma, se segmente également; si l'on roit une cette segmentation progresse juscyüa un certain point, on n'a pas le droit d'en conclure que le déreloppenent se pumsuivra de la mêne manière jusqu’a la formation complète de l'embryon. Nons arons ru que la segmentation n'est que l'effet de l'assimilation. Fille nous démontre lonc que l'assimilation s'est accomplie dans les cellules qui se dirisent; mais cela n'implique nullement qu'elle se poursuive dans les autres cellules qui en dériveront, et, par suite, que le phénonène du déreloppenent de l'embryon, lébutant par la segmentation, loive se continuer jusqu'a l'achèvement. Elle pourar, au contraire sarrêter à une phase quelconque de lérolution de l'nenf: et si cette phase n'est pas la phase linite, le déreloppenent ultérieur sera naturellenent empêché.

On peut whjecter ici que non seulement lat segmentation a lieu, mais qu'il se forme aussi une blastula typique, tout comme dans le léreloppement le loeuf entier. 'ette objection est sans valeur: car nous sarons que cette formation morphologique nest que la conséquence mécanique le la segmentation et le l'accunnlation d'un liquide a l'inférieur de la cavité de segmentation.

Or, comme je viens de démontrer que l'a-inulation doit se faire également dans les neufis excisés. pourru qu'ils contiement encore des substances deutoplatsmatiques, quoi de plus naturel que lat sécrétion de suhstancess speciales, conséquence directe de lassimilation, se fasse, elle anssi, mormalenent, et que ces substances s'accumulent it l'intripieur de longrégat cellulaire dérivant de lat sermentations, tout commue dans le déreloppement normal des oeuf's?

La formation d'une blastula typique, pas plus que la segmentation, nimplique donc point le développenent normal ultérieur jusqu'à l'achèvement de l'embryon.

Il nous reste maintenant i cunsidérer le rolume et le nombre 
des cellules dins les embryons dérivant des oeufs excisés. Quant au volume, il est érident que, comme l'exeision d'une furtion de l'oeuf aminue le rolume total de celui-ci, les cellules dérivant de sal sermentation présenteront, elles anssi, une diminution correspondinte: cin nous savons que, dans les oenfs it segmentation totale, le rolume des blastomères n'est qu'une portion du rolume total de l'oeuf. Si donc on compare les blastomères d'un embryon normal arec les blastomères d'un embryon dérirant d'un oenf excisé, on trouvera toujour's une diminution de rolume, correspontant it la diminution qu’a subie l'oenf. Cependant cette dillẻrence de volume n'existera pas it toutes les phases du développenent, et nous pouvons facilement nous en convaincre.

Le rolume total d'une cellule de segmentation est égal i la somme du volume du bioplasma et du volume des substances deutoplasmatiques qu'elle contient; et si cette cellule, dans les cats d'ootomie, est plus petite, c'est que lat masse deutoplatsmatique a subi une diminution; mais le volume lu bioplasma est toujours parfaitement égal à ce qu'il était normalement. En d'autres termes, la cause le la diminution du volume n'est pas dams la diminution du bioplasma, qui reste toujours le mème, mais dans le reutoplasma qui a été soustrait à l'oeul:

Par conséquent, tant que les cellules de segmentation et les autres qui suirront dims le déreloppement ultérieur, contiemlront des substances deutoplasmatiques, leur volume sera moindre que le rolume des cellules correspondantes dinss un embryon normal et à la mème phatse du déreloppement; mais. dès que les cellules ne contiendront plus de deutoplasmat, celui-ci ayant été absorbé peu à peu par l'assimilation du hioplasmat, leur rolume total sera égal au volume des cellules correspondantes dans les embryons normaux et à la même phase du développement. La différence dans le volume des cellules entre les embryons normaux et les embryons provenant d'oeuf's excisés, disparaîtra donc à une certaine phase de leur développement. 
Quant an nombre des cellules constituant lembryon issu d'un veuf excisé, nous pourons le déterminer avec une précision mathématique, pourru que lat phase du développenent a laquelle nous considerons les cmblyons puisse itre, elle anssi, léterminée avec la plus gramle précision possible: ce qui d'ailleurs peut grésenter des linleultés assez grares.

si nous pourions comantre patr yuelques canactères visibles lat phase limite le l'érolution le l'oeuf, nous pourroms choisir, comme terme de comparaison entre les emingons nomaux et les embryons d'oeufs excisés, le noment précis ou cette phase est atteinte par un des blastoneres. Mais, celat n'étant pas possible arec les morens actuels de recherche, l'on doit s'en tenir à un caractère morphologique aisénent reconnaissable. Lit comme, dans la plupirt des car, la première modification morphologique bien visible qui saccomplit apres la sexmentation ext loinvagination, qui dome lien a la gastrula, nous jourons choisir, comme terme le comparatison entre les embryons, l'instant même où cette invagination commence.

Nous arons ru, au chapitre VIII, que la gastrulation par invagination est un phénonène mécanique défendant de l'absorption des substances contenues daus la carrité de la blastulat de lat part des cellules de ha deuxieme lignere at que ces cellules dérivent du histomere anrivé à la phase linite de l'évolution de loeuf: Mais comme cette tormation de la denxième lignée cellulaire ne se fait pas ausitrit qu'un hatstomère ar-

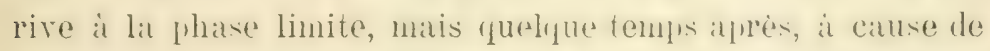
la périorle assintatrice nécessitipe all halstomère mètue pour se diviser; vu, d'autre part, que le phénomène norphologique de l'invagination ne pent se rembre manifietre que quelque temps après le commencement de lat formattion le la denxiome lignée cellulare, nous devons truir compte que, si linstant choisi peut servir à notre but dans certaines limites, il ne

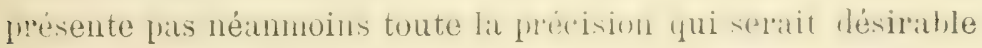
pour la comparaison des résultats qui nous intéressent.

Si donc, entre les résultats thériques que nous pouvons 
déterminer arec une précision mathématique et les résultats obtenus par les expériences, il n'y a pas une concondance parfiatement exacte, cela est dii à l'impossibilité ou nous sommes de comparer les embryons dans une même phase de leur déreloppement.

Nous arons vu, au chapitre VII, fig. 13, que l'agrégat cellulaire, dians l'așrnchronisme accéléré, it l'instant qu'apparaît un blastomère it la phase limite 1 , est constitué de 62 cellules, ot qu'au même instant, dans l'asynchronisme ralenti, les cellules de l'agrégat sont 13:2 (fig. 19), c'est-i-dire que le nombre. les cellules est dépendant, ainsi que je l'ai démontré, de l'asynchronisme et du rythme de segmentation. Or, comme l'excision d'une portion de l'oeuf, pourru qu'elle ne soit pats trop forte, ne peut influer ni sur l'asynchronisme, ni sur le rythme de segmentation, nous pourons conclure que l'agrégat cellulaire et, par conséquent, l'embryon résultant du déreloppement d'un oeuf excisé, doit présenter le mème nombre de cellules yu'un embryon issu d'un oeuf normal et considéré it la même phase de développement.

En conclusion, nous pouvons établir que si un oeuf est

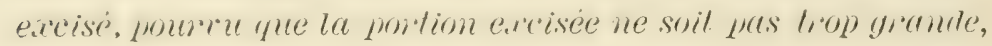

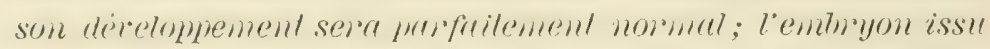
de cet oeuf sera complet, et le nombre des cellules sera le même que dans les embryons nomaux considèiés ì la mêne phase du développement; mais le volume des cellules de segmentation sera d'autant plus petit que le volume de l'oeuf aura élé diminué par l'excision.

Ces résultats, obtenus théoriquement en partant des principes de mon interprétation, concordent très bien arec les résultats des expériences de $\operatorname{Ror} x(1)$ et le Moradx (2). (es

(1) Roux WV. - Beiträge zur Enhwiolelungsmeohanili des Embryo. Einteitung und 1. Zur Orientinug über einige Probleme der Embryonalen Entwickelung, in: Zeit. Biol., 21 Bil, 1885.

(2) Morgan T. H. - Studies of the " Partial Larvas of Sphaerechinus, in: Arch. f. Eutwick., II Bd., 1896, pp. 81-126. 
deux sarants Biologristes ont constaté en effet, dans leurs expériences, que les embryons issus d'oeul's ootomisés, quoique plus petits, ne sont pas moins complets que les embryons normaux.

Quant au nombre des cellules constituant les embryons, les résultats obtenus par Morkin ne concordent pas parfaitement arec les résultats théoriques que je viens d'énoncer. Nais ici il fiut tenir compte que, dans la plupart des expériences de MoraAn, les fragments d'oeufs éticient peut-être trop petits et, par suite, au-dessous de la taille minima nécessaire pour le léreloppement normal, ainsi que je l'ai démontré. Cependant, on peut constater, par les mêmes expériences de Morgan, que, dans les larves provenant des fragments les plus grands, le développement et le rythme de segmentation étaient normaux et le nombre des cellules a peu pres égall à celui des larves normales.

Il faut d'ailleur's que de nurelles experiences solent faites sur cet intéressint sujet, pour nous permettre d'arriver à des résultats plus précis.

\section{§II.}

Sur le développement des blastomères isolés.

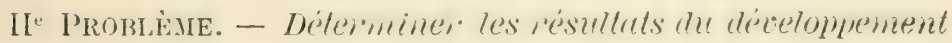
des deux premiers blastomères isolés.

Solution. - C'est surtout dans ces expériences que nous purrons constater l'aptitule de mon interprétation it donner une explication scientifique, de la plus grande simplicité et de la plus rigoureuse précision, de phénomenes aussi énigmatiques qu'intéressants, et qui n’ont reçu jusqu’a ce jour que les tentatives d'interprétation très hrpothétiques et peu scientifiques.

Revenons a l'exemple cité de léreloppentent normal et supposons donc que l'oeuf ", s'itant divisé en leux blastomères $b, c$, ceux-ci soient parfailement isolés l'un de l'autre. 
Y a-t-il des raisons plausibles pour supposer que ces blastomires ne poursuivent pas leur segmentation? Je ne le crois pas. Doit-on supposer yuapris l’isolement interviement des forces, ou des conditions, ou des mouvements internes permettant ou déterminant leur segmentation? Je ne le crois pas non plus. Ce serait une supposition tout-i-fait gratuite et nullement scientifique.

Quoi d'ailleur's de plus naturel et de plus simple que d'admettre que les blastomères isolér se comportent parfaitement comme s'ils étaient réunis?

Considérons les deux blastomères $b$ et $c$, et royons à quels résultats théorinues nous pouvons arriver sans l'intervention d'ancun autre principe que le développement monodique, uni est la hase de mon interprétation. I)ans ces considérations, je supposerai, pour plus de simplicité, que les blastomères soient éganx, c'est-it-dire dérivés d'une semmentation totale et égale. fous rerrons peu après quelles modifications peurent dériver dans les cas de segmentation partiale et inégale.

$1^{\circ}$ ) Le blastomère $b$, né a 2 heures, après 1 h. 55', durée de la périole assinilatrice de son bioplasma, se dirisera, tout comme s'il était uni à l'autre blastomère $c$, en deux autres blastomères $c$, $d$. Ce premier plan de segmentation aura donc lieu it 3 h. $55^{\prime}$.

Ces deux blastomères se diviseront, ì leur tour, après leur périorle diassimilation, et la segmentation se poursuirra réfrulièrenent, comme nous l'arous exposes au chalpitre VII, dans le développement normal; et si le blastomère 7 , après l'isolement, a repris sa forme sphérique comme nous le supposons pour plus de simplicité, et comme il arrive en efret presque toujours à défaut de l'adhésion à l'autre blastomère, qui en arate molifié la forme sphérique primitive, les plans de semmentation se disposeront conme dans l'oent complet, d'apris les déterminations que l'on trouve exposées au chapitre IX de la I Partie.

A 10 h. 30', c'est-à-dire à l'instant où, dans l'oeuf complet, 
le blastomère le plus arancé atteint lia phase limite ple l'évolution de l'oeuf, l'agregat cellulaire résultant de lat semmentation du blastomère isolé 7 se trouvera constitué comme nous le royons représenté dans la fig. 20, où l'on peut constater que le blastomère le plus arancé n'a atteint que

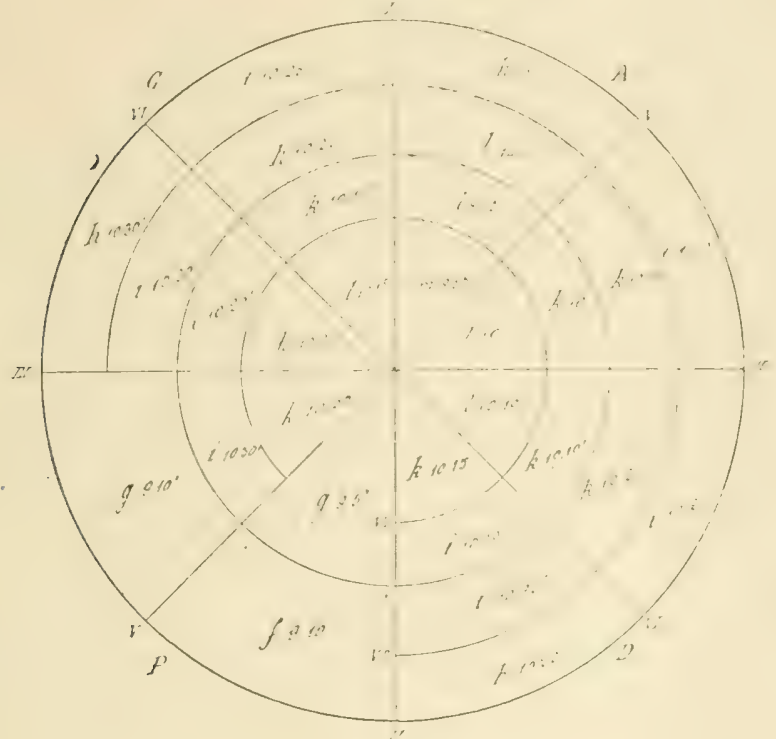

Fig. 20.

la phase $m$ de l'évolution de l'oeuf. Par rapport au développement de l'oeuf complet, le lérehnpement du blastomère b est donc en retard. A ce moment, le nombre des cellules de l'agrégat cellulaire est de 29.

Evidemment lat somentation progresseril, pourvu, bien entemlu, que la quantité des substances deutoplatsmatiques nécessabes a l'atsimilation soit sulfisante, of lat phatse limite p ne sera atteinte qu'i 11 h. 55', c'est-ì-dire 1 h. $255^{\prime}$ plus tard que dams l'vent complet. I ce moment, l'agrégatt cellulatre est composé de 59 cellules c'est-it-lire de 3 cellules de moins que 
l'agrégat resultant de lit segmentation de l'oeuf complet. Cet agrégat est représenté dans la fig. 21.

$2^{\circ}$ ) Considérons maintenant le blastomère $c$.

Ce blastomère, né, lui aussi, ¿̇ 2 h., se divisera à 3 h. $50^{\prime}$ en deux blastomères 1 , e, et ceux-ci i leur tour se diviseront,

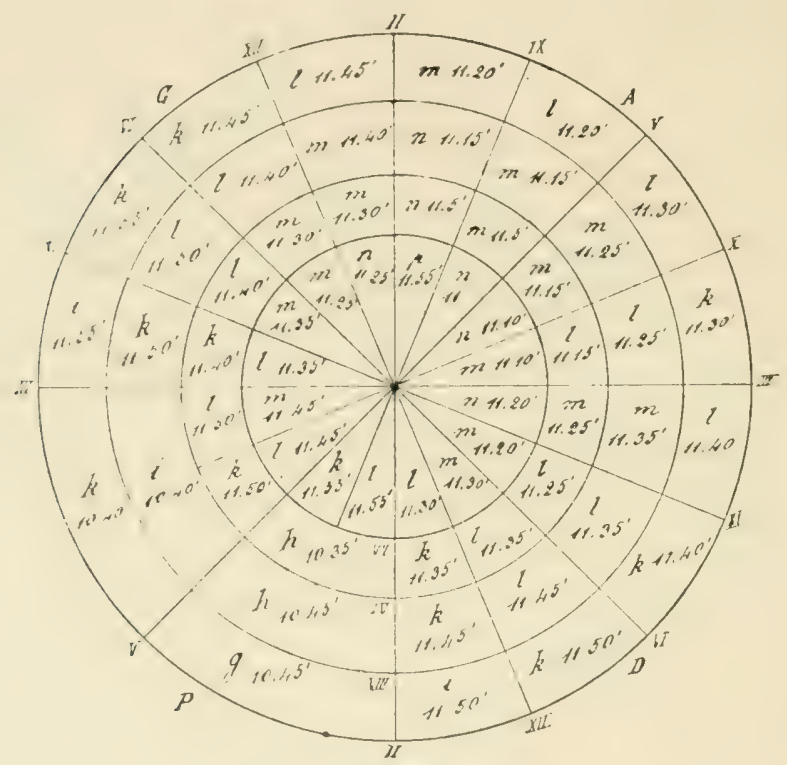

Fig. 21.

et la segmentation progressera tout comme dans l'oeuf complet. A 10 h. 30', le blastomère le plus avancé atteindra la phase limite $p$ de l'évolution de l'oeuf, c'est-i-dire qu'il arrivera à cette phase de l'évolution au même instant que l'oeuf complet. I ce moment, l'agrégat cellulaire sera constitué comme nous le vorons dans la fig. 2.2, et le nombre de ses cellules sera de :33, c'est-it-dire qu'il possèdera à peu près la moitié des cellules que possede l'arrégat lérivant de l'oenf complet considéré au même instant du développement.

Or, si nous comparons la constitution des deux agrégats 
cellulaires résultant de la segmentation des deux premiers blastomères isolés arec la constitution de l'agrégat dérirant de l'oeuf complet, nous verrons que cette constitution, sanf le nombre des cellules, est la mème, il cette exception près que, dans l'agrégat de l'oeuf' complet, le hlastomère fe moins arancé

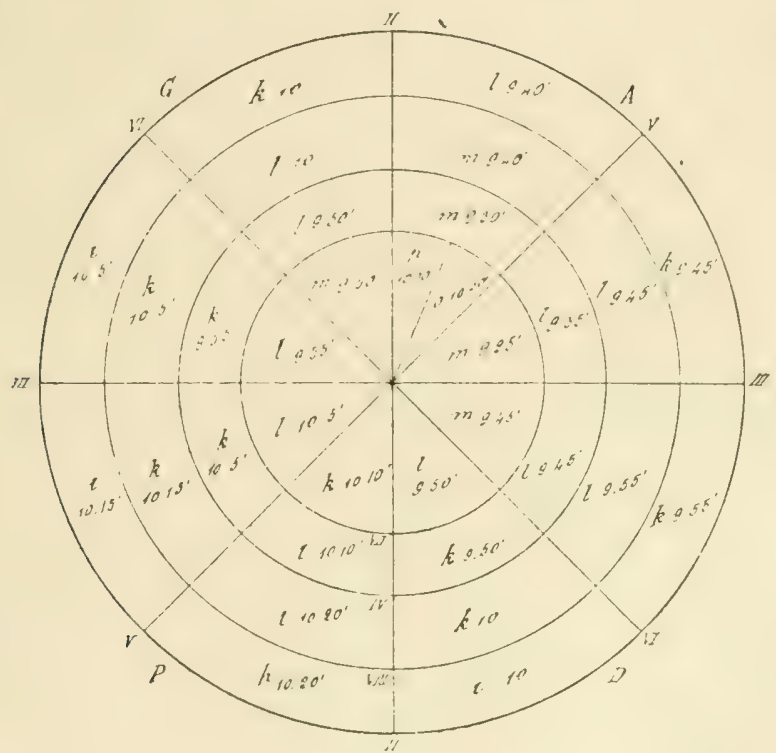

Fig. 22.

se trouve encore, a $10 \mathrm{~h}$. :30, dans la phase f, tandis que, dans les agrégats dérivant les blastomères isolés, cette phase a déjá disparu à l'instant oi la phase limite /l est atteinte, et plus précisément, que dans l'agrégat lérivant du blastomère 1, la phase la moins arancée de l'érolution le l'neuf est g of que, dans l'autre agrégat dérivant du blastomère ', la phase la moins avancée est $h$.

Mais comme le développement ultérieur est déterminé par la prolifération l'autres cellules provenant du blastomère le plus arancé, dont l'assimilation se fait aux dépens des suh- 
stances sécrétées par les blastomères et accumulées à l'intérieur de l'agrégat, il est érident que le développement se poursuira normalement. En eflet, puisque la constitution

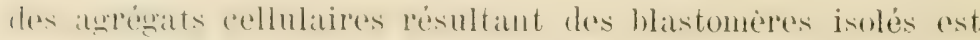
substantiellement la même que celle de l'agrésat de l'oeut complet, les substances siorétées seront, elles anssi, les mimes:

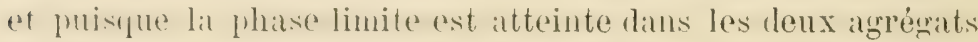
susdits, ces deux agrégats se trourent donc dans les mêmes conditions que l'agrégat complet. Il n'y a donc pas de raisons pour supposer que le léreloppenent ultériem ne puisise aroir lieu. Par conséquent, l'embryon qui en résultera présentera parfaitement la mème symétrie quue l'embryon dérivant de l'oeut' complet.

Cependant, il faut ici considérer que, si ces agrégats contiennent un nombre de cellules moindre que l'agrégrat issu de l'oenf complet, cela est dû à l'isolement des blastomères. Si l'agrégat dérivé du blastomère $c$ n'a qu'à peu près la moitié des cellules de l'agrégat normal, la cause en est à ce fait que, par l'isolement de ce blastomère, nous arons soustrait a l'agrégat toutes les cellules dérivant des divisions du blastomère $b$ et des descendants de celui-ci. Et si l'agrégat dérivé da b possède un nombre de cellules à peu près normal, quoique trujumrs moinlre, c'est que, par l'isolement, on a bien soustrat de l'agrégat toutes les cellules dérivant de la segmentation de $c$; mais comme le développement de ce blastomère $b$ est en returd par rapport au développement normal, le nombre des cellules se rapproche du nombre normal par les divisions s'accomplissant pendant 1 h. 25 , qui représente la valeur de ce retarl.

En d’autres termes plus généraux: si le nombre des cellules est plus petit que le normal dans le cas d'isolement des blastonnirres, cest que charue histomere isolé, considér comme un oeuf, doit parcourir un chemin plus court pour arriver it son but. Nais cette abréviation n'existe pas pour l'évolution des cellules des autres lignées. 
Tous avolls, pall exemple, supposes yue les phises de l'éro-

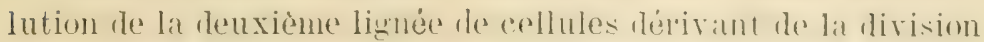
du blastomère $p$ sont $b^{\prime}, c^{\prime}, d^{\prime}, e^{\prime} \ldots p^{\prime}$. Évidemment, dans les conditions normales, lorspue la phase fi sera atteinte pill une des cellules, lo nombre lles cellules de la 2) lignée serar léterniné et rependiunt du mombre des phases et du rythme de division. Or, dius le développenext des hastomères isolés, le point de départ pour la formation le la 2 lignée est le blastomère $p$, et les phases de l'évolution sont les mèmes que nous venons d'indiquer', tout comme dans les conditions normales. Par conséquent, le nombre des cellules de la ₹ lignée, dans le développement des blastomères isolés, sera le mème que dans le developpement de l'oeuf complet.

Cependant il fiut ici tenir compte d'un antre tait, qui ne prut itre négligé pour la comprehension exacte des phémmuèmes.

Tous sarous que le déreloppement des cellules de la 2. lignnée se liat aux rejents ressubtancess sécrétées par les blastomères. Or, si le nombre de ceux-ci est plus petit, les substances se tromveront, alles ansi, an quantite moindre que normalement.

Nous avons vu en outre que la 2 e lignée de cellules, ainsi que les autres suivantes, ne sont pas des liggnées simples, mais multiples. J'al démontré en eflot, au chafitre $\mathrm{X}$, qu'après que lạ formation d'une 2 lignée cellulaire a commencé, d'autres blastomeres arrivant, eux anssi, il la phase $\mu$, point de départ pour cette lignée, suirront cette nouvelle érolution et, par conséquent, deux on plusions sipies des cellules se tronveront dirigées rers la mỏme phite limite fí de la 2 " lignée cellulaire.

Mais comme les cellules de cette nourelle lignée puisent leur nourriture dans le milieu interne, c'est-i-dire dans les substances sécrétés par les hastomeres, il es divlent que

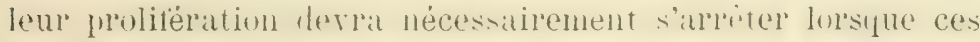
substinces nourrissantes seront épuisés, et pall conséruent, lo

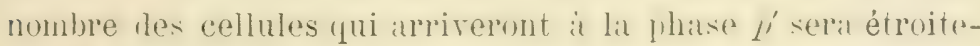
ment répendant de la quantite des substances nourrissintes du milieu interne. 


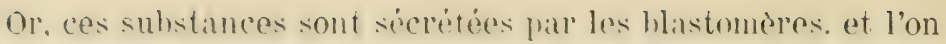
comprend fitcilement que, si ceux-ci sont moins mombreux, les sulfances aussi seront moins abondintes. Nous pourons, par (a)tséquent, en déduire que, dans le développenent du hlastomère isolé $/ 1$, ou les blastomères dérivant de sal semmentation sont i feine yuelyue peu moins nombremx que normalement, les cellules aussi qui arrireront i la phase f' seront à peine moins nombreuses que dins le cas de développement de l'oeuf complet; tandis que, dans le cas du blastomère isolé ce, le nombre des blastomères dérirant de sa segmentation étant a felu près la moitie du nombre normal, les substances sécrétées seront, elles ausi, en quantité à peu près moitié de la normale, et les cellules arrivant a la plotse $y^{\prime}$ seront en nombre a peu piès moitié du normal.

cette déduction logique que nous venons d"appliquer aux collules de la deuxième lignçe, nous pouvons l'étendre aux cellules des autres lignées et, par suite, aux cellules consti-

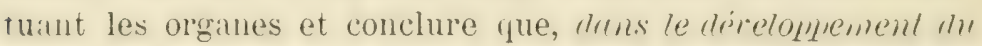

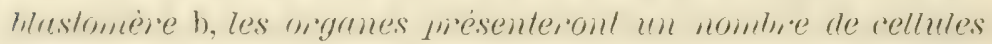
quelque peu inférieu" au nombre normal, tandis que, dans

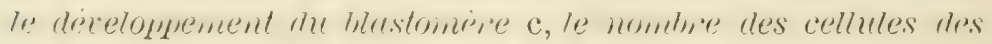
niganes sera à peu près la moitié du nombre nomal.

Guant au rolume des cellules constituant les embryons rérivés du développement des blastomères isolés, il est évident qu'il ne pourra être différent du volume normal.

Nous conclurons donc que tous les deux memiers blastowières isolés se développent noimalement et pervent donner. lieu i la fommation d'un embryon complet, dont les cellules ont le meine colume que dans l'embryon provenant de l'oeuf

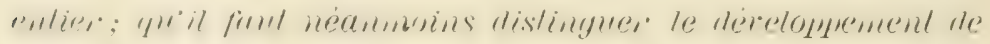
ces deux blastomires: car dans l'un, l'achèrement de la segwentation est quelque peu retardé, et la phase de la gastrulation est alleinte loisque les cellules de l'agrégat de segmenfation sont quelyue peu inferientes au nombre nomal, tandis que, dans l'autre, l'acherement de la sergnentalion n'est jas 


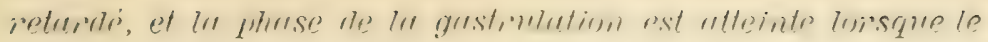

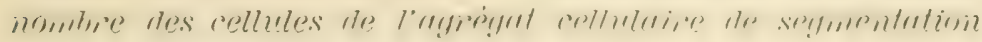
est a peu press la moitie du nombre novmal; que le nombre des cellules des arganes, dans ce dernier cas, doit être à peu pì̀s la moitié du nombire des cellules constituant les mêmes

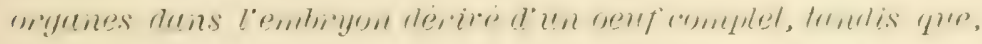

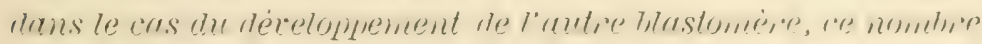
sera ci peine quelque peu inférieur au nointhie normal.

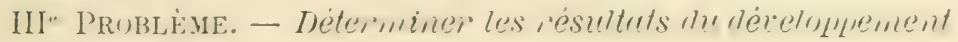
des quatie memiers blastomères isolés.

Solutiox. - La solution de ce probleme est aussi simple ¡ue celle du probleme précident. Ici encore, il n'est mas nécessaire de supposer des phénomènes spéciaux. Les quatre hlastomères isolés se lévelupreront parfaitement comme sils étaient réunis.

Revenons donc a notre exemple, oi les yuatre blastomeres r, r, 1, e ifig. 3) constituent lagrégat cellulaire arres le $\mathrm{I}^{\mathrm{a}}$ plan de segmentation, et considérons-les séparément.

1) Le blastomère $c$, né à 3 h. 55', se divisera, ¿̇ 5 h. 45', en 1, e, et ceux-ci poursuirront rérulièrenent leur segutentation. A 12 h. 2̌’, un des blastomères arrivera i la phase linite $l^{\prime}$ et, it ce moment, l'agremat cellulaire le segmentation sera constilué comme il est représenté dans la fig. 23, et posiedera :3:3 cellules, cest-it-dire it feu rorés la moitié du nombre normal.

Comparons maintenant cet agrégat arec les autres dérirant de la segmentation de loeut complet et des leux preniers blastomères isoles, et noms en verrons resortir les fats tris intéressants et presque inattendus.

Sous pourons constater arant tout yue, dans ce cas, la phase limite de l'érolution de l'oent' est atteinte une heure mó flus tard que dans les déreloppements lo l'veuf complet et du hlastomère $c$ isolé à la phase les reux preniers blastomères, et 30 minutes encore plus tard que lans le déreloppenent du 
blastomere $b$ isolé. Le retard subi dans ce cas pour atteindre la phase limite de l'érolution de l'oeut est done encore plus grand que dans le cas précédent.

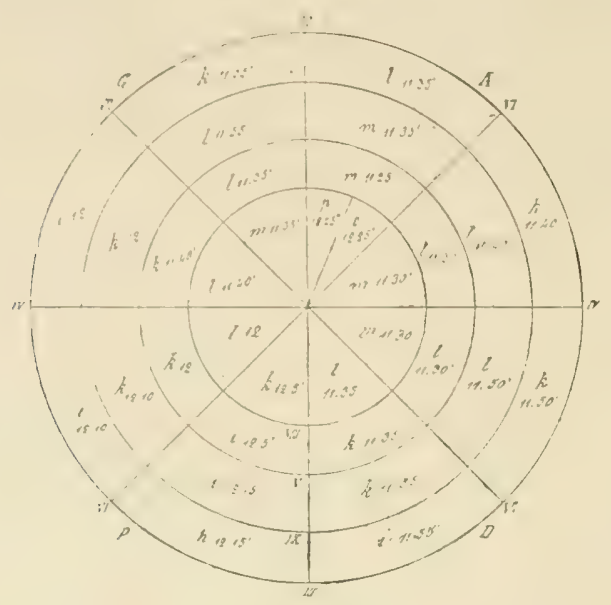

Fig. 23.

En second lieu, si nous comparons la fig. 23 avec la fig. 22, nous pourons constater que la constitution de ces deux agrégats cellulaires est parfaitement identique par rapport soit an nombre des cellules, soit it la nature de celles-ci, mais que, dans l'agrégat de la fig. 2:3 dérivant de $\frac{c}{t}$, (1) tous les blastomeres sont de 1 h. $55^{\prime}$ plus jeunes que les blastomères correspondants dans l'agrégat de la fig. 2.2 dérivé de $\frac{c}{2}$.

$2^{\circ}$ ) Le blastomère $d$, né à 3 h. $50^{\prime}$, se divisera, à 5 h. $35^{\prime}$, en $e, f$ ot ceux-ci poursuivront régulièrement leurs divisions

(1) Pour plus de brièveto, jontends par $\frac{b}{2} \frac{c}{2}$ les blastomères $b$, e isolés $\therefore$ la phase de denx blastomeres; par $\frac{c}{4} \frac{d}{4} \frac{e}{4}$ los blastomeres $c$, d, e isoles à la phase do quatre blastomeres, etc. 
ultérieures, de sorte qu’a $11 \mathrm{~h}$. 50', un blastomere arrirera à la phase limite ple l'érolution de l'veut. A ce moment, l'agrégat cellulaire re segmentation seril constitue comme nous

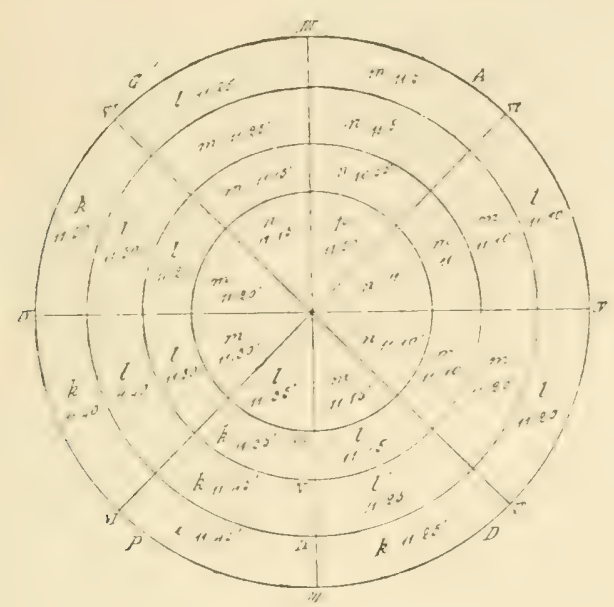

Fig. 24.

le royons représenté dans la figr. 21. Les cellules seront au nombre de 32.

Dans ce cas, la phase limite est atteinte 1 h. $20^{\prime}$ plus tard que dins le développenent de l'oeuf complet et du blastomere $\frac{c}{2}$, 5 minutes seulement plus tît que dans le dérelopuenent de ${ }_{2}^{-}, 35$ minutes plus tôt que dans le déreloppement de $\frac{c}{4}$. Le nombre de cellules est le mème que daus l'agrégat dérivant de la segmentatiou de $\frac{c}{f}$, c'est-it-dire it peu près la moitié du nombre normal.

$3^{\circ}$ ) Le blastomère $d$, né ì 3 h. 55', c'est-ì-dire 5 minutes seulement plus tard que le frécédent, donnera évidemment lieu, par sa segmentation rérgulière. ì un agréégat cellulaire identique à celui de la fir. 2t, dont toutes les cellules seront 
néammoins plus jeunes de ä que leurs correspondantes de l'agrégat de la fig. 21. Aussi la phase limite de l'évolution de l'oeuf ne sera-t-elle atteinte qu'i 10 h. 55 '.

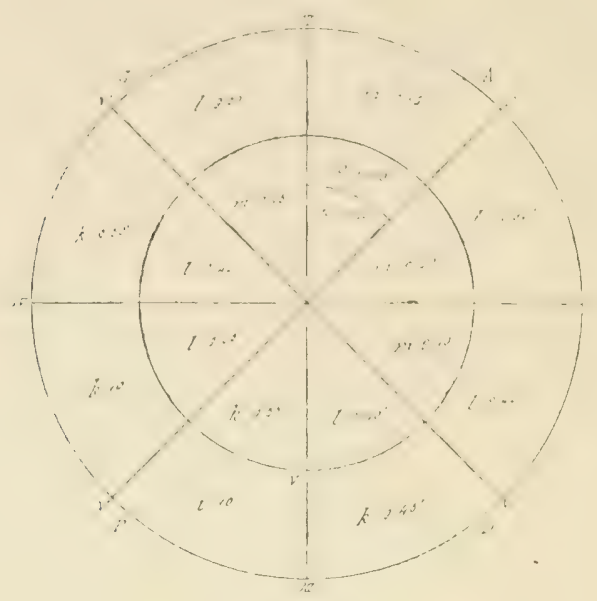

Fig. 25 ,

$\left.4^{\circ}\right)$ Enfin le blastomère $e$, né ì $3 \mathrm{~h} .50^{\prime}$, se divisant régulierement, Iomera origrine it un agremat cellulaire tel que nous le représentons dans la fig. 25 , où la phase limite $p$ est atteinte à 10 h. $30^{\prime}$ c'est-à-dire ì l'heure même où elle est atteinte dans le léveloppement de l'veuf complet. Dans ce cas, le mombre des cellules de cet agrégat cellulaire n'est que de 17, c'est-à-dire à peu près $\frac{1}{4}$ du nombre normal.

Si maintenant nous comparons entre eux ces quatre agrégats cellulaires dérivant du développement des 4 premiers blastomères, nous royons que, it part le nombre des cellules, leur constitution est la même et qu'elle n'est pas' substanliellenent dinérente de la constitution de l'agrégat dérivant Jr la semmentation le l'oeuf complet. Par conséfuent, en parlant toujours des principes de mon interprétation de l'ontogringas. an peut conclure ot démontrer logiquement que le 
développement ultérieur de ces quatre hlastonères donnera orizine it un embryon complet, quoique proportionnellement plus petit. Ici encore, on roit clairenent que cette conclusion n'est qu'une conséquence logique et inéritahle dec principes de mon interprétation.

Quant au nombre des cellules des organes de l'embryon, nous pouvons fatre ici des considérations analognes a celles que je viens de faire au II problème.

En supposant que, la dirision etant égale et totale, les quatre premiers blastomères soient éraux, la quantité de deutoplasma contenue dans chacun d'eux sera naturellement $\frac{1}{t}$ de la yuantité totale contenue dans l'oeuf complet.

Mais comme le nombre des cellules des organes qui se forment pendant les prolifërations ultérieures dépend, en partie au moins, de la quantité de substances nourrissintes contenues dans le milieu interne; comme d'autre part, ces substances sont proluiter par les cellules mines précédentes et sont en quantité proportionnelle au nombre de ces cellules, on comprend facilement que, dans les agrégats cellulates dirivant des blastomères $\frac{c}{4}, \frac{d}{4}\left(3 \mathrm{~h} .50^{\prime}\right), \frac{d}{4}\left(3 \mathrm{~h} .55^{\prime}\right)$, où le nombre des cellules de segmentation est ì peu près la moitié du normal, les substances sécrétées par cescellules seront, elles aussi, i peu près la moitié de la quantité normale. Par conséquent, les organes présenteront un nombre de cellules à peu près la moitié du normal.

Au contraire, dans le développement du blastomère $\frac{e}{4}$, le nombre des cellules de segmentation est semlement it fent pris $\frac{1}{4}$ du normal, et la quantité des substances sécrétées frar cess cellules sera donc, elle aussi, $\frac{1}{4}$ de la quantité normale. Par conséquent, le nombre des cellules des orranes de l'embryon sera, dans ce cas, $\frac{1}{4}$ seulement du normal. 
Nous pourons donc conclure que, si l'on isole les quatre

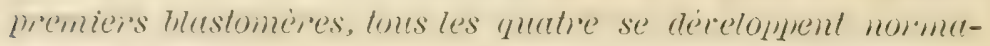

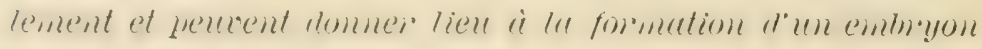
complet, dont les cellulles ont le mêne volume que dans l'embiyon provenant de l'oeuf entier; qu'ici encore, il faul néan-

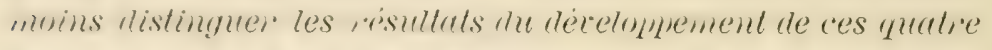

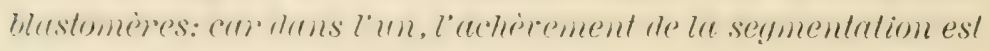
de beancoup retardé; dans deux autres, il est moins en retard et duns le quatrieme, il ne l'est pas du tout: que, dans trois de aes blastomires, le nombre des cellules de segnentation (it la

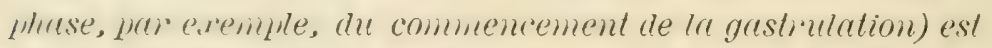

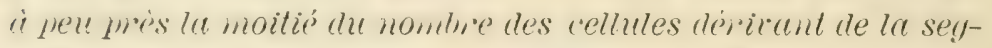

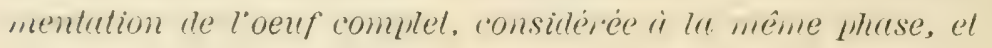
que dans l'autre, ce nomure est à peu près $\frac{1}{4}$ du nomal; et que. pur conséquent, celle mrine jroportion pouna être conslatée entre les nombres des cellules des organes.

IV PRoBlíme. - Délerniner les iésultuts du déreloppenent des huit premiers blastomères isolés.

Solution. - Les huit premier's blastomères sont (figr. 5) d, $e\left(5 \mathrm{~h} .35^{\prime}\right), e\left(5 \mathrm{~h} .40^{\prime}\right)$, e (5 h. $\left.45^{\prime}\right), f\left(5 \mathrm{~h} .30^{\prime}\right), f\left(5 \mathrm{~h} .35^{\prime}\right)$, $f\left(5\right.$ h. $\left.40^{\prime}\right), y$. Considérons leur développement séparément, comme nous l'arons fait dans les problemes préciedents.

$\left.1^{\circ}\right)$ Le blastomère $d$ isolé, se développera, lui aussi, normalement, et un des blastomères dérrivant de sa segmentation arrivera à la phase limite $p$ à 13 h. 45', c'est-ḋ-dire 3 h. 45' plus tard que dans le développenent de l'oeuf complet. A co moment, l'agrégat cellulaire résultera constitué comme il est représenté dans la fig. 20. Ses cellules seront au nombre de 32 , c'est-ì-dire ì peu près la moitié du nombre normal, et égal au nombre des cellules des agrégats dérivant de $\frac{c}{2}, \frac{c}{4}, \frac{a}{4}$.

$2^{\circ}$ ) Le blastomère $e$, né à $5 \mathrm{~h}$. 35 , se développant normalement, donnerat origine à un agrégat cellulaire comme nous 
le voyons représenté dans la fig. 27. Isil phaso limite pl est atteinte a $12 \mathrm{~h}$. 15', et, par suite, $1 \mathrm{~h} .45^{\prime}$ plus tard que dans le développement de l'oeuf complet. ses cellules seront au nombre de 17 , c'est-it-dire a peu près $\frac{1}{4}$ du nombre normal.

30) Le blastomère $e$, né i $5 \mathrm{~h}$. $4 \mathrm{y}^{\prime}$ donnera lieu éridemment a un agrégat cellulaire identique au précérlent. Les cellules

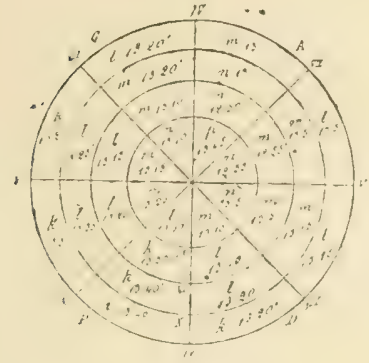

Fig. 26.

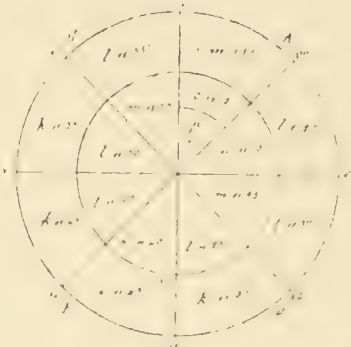

Fig. 27.

seront néanmoins plus jeunes de sminutes et la phase limite sera, par suite, atteinte it 12 h. $20^{\prime}$.

$\left.4^{\circ}\right)$ Le blastomère $e$, né it 5 h. 15', produira un arréégat cellulaire identique au précédent. Cellules plus jemes de à minutes. Phase limite atteinte à 12 h. $25^{\prime}$.

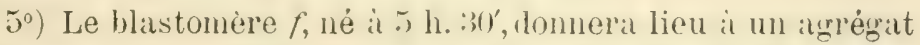
cellulaire conme il est représenté dans la fig. 20. Tombre des cellules 16. Phase limite atteinte it $11 \mathrm{~h} .45$.

$\left.6^{\circ}\right)$ Blastomère $f$, né à 5 h. 35'. Agrégat cellulaire constitué comme le précédent. Toutes les cellules de j) minutes plus jeunes. Phase limite atteinte à 11 h. 50 .

70) Blastomère f, né a jo h. 10'. Agrégat cellulaire constitué comme le précédent. Cellules de in minutes plus jeunes. Phase limite atteinte à 11 h. $55^{\prime}$.

8") Enfin le blastomere y, né it 5 h. 319, donnela lieu, par sa segmentation normale, it un argrégat cellulaire tel qu'il est 
représenté dans ia fig. 29, où l'on voit que la phase limite est atteinte a l'heure normale, it 10 h. 30', lorsque le nombre des cellules est it peine de 9, cest-it-dire i peu pres $\frac{1}{8}$ du nombre normal.

En répétant ici les mèmes considérations qu'aux solutions des problemes précédents, nous pourons arguer que tous ces

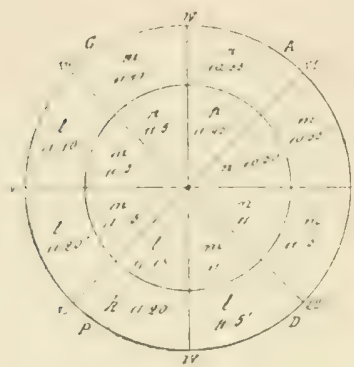

Fig. 28.

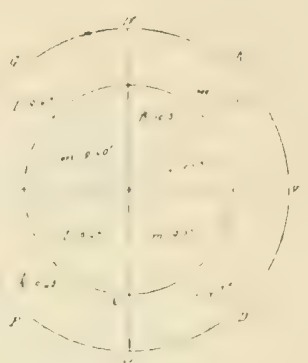

Fig. 29.

blastomères isolés doivent donner origine, par leur développement, a un embryon complet, et, par suite, a huit embryons.

Yous arons donc, ici encore, le droit de conclure que les hnil premiers blastomères isolés se développent nomalement et

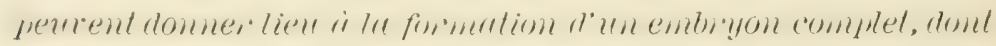
les cellules ont le même rolume que dans l'embryon nomal; que dans un de ces blastoméres, l'achèvement de la segmenlation subit un retard hrès accentué, et dans ce cas le nombre des cellules de segmentation est it peu près la moitié du normal; que dans trois autres blastomères, le retard est moins accentué, et le nombre des cellules de segmentation est à peu j)ès $\frac{1}{4}$ an nomual; que dans trois autres, le retard est moins accentué encore et le nombre des cellules de segnentation est encore ì peu près $\frac{1}{4}$ du normal; enfin que dans un seul de ces huil blustomeres, lacherement de la sergnentation se fait 
stins iretard, et dans ce cas, le momitive des rellules de semmontation est à peu jiès $\frac{1}{8}$ du nomal; que, par conséquent,

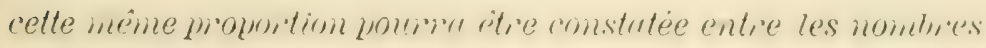
des cellules des organes.

Je ne crois pas nécessaire d'exporer les résultats du déreloppement des 16 ou des 32 premiers blastomeres isolés; car les solutions que je riens de donner des froblèmes précédents sont sullisantes pour nous lémontrer que quel que sott le nombire des hustomires, centi-ci, isolés, se derelonpent toujouris

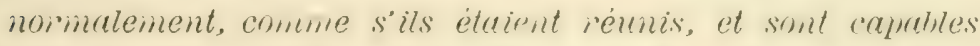
de donner lieu, pur leur dévelopturenent, is lu. formation d'un embryon complet, pourvu, bien entendu, que lat substance deutoplasmatique contenue dans chacun d'eux ne snit pas inférieure au minimum nécessaire au déreloppement.

Quant au nombre des cellules constituant l'agrégat cellulaire a l'achèvement do la segmentation, fout hologiste pourra, avec la plus grande facilité, la déterminer dans tous les cas arec précision en suirant le processus tric simple que j'ai suiri moi-mèn e lans. les cas précédents, c'pst-it-lire en suirant le développement monodique et en tenant un compte exact des durées des périodes d'assimilation des cellules.

Maintenant, si nous comparons les résultats obtenus par less solutions de ces problemes, nous pourons constater ces fats très intéressants: que, parmi les blastomères isolés, il n'y en a qu'un seul qui ne subisse pas de retard dans son développement et que, dans ce cas seulement, le rapport entive le

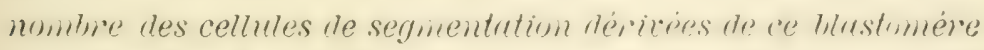
et le nombie des cellules de segmentation dérivées de l'oeut

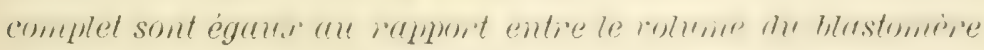
el le volume de l'oeur; que dans tous les autres blastomères, on constale un retard dans leur déreloppement; que ce retard est de plus en plus accentué, a mesure que les blastomères isolés sont plus nombreux; que, dans ces cas, le rupport entre

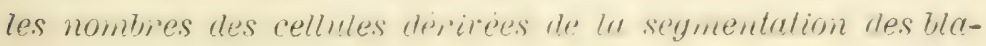




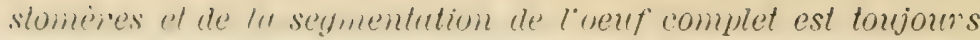

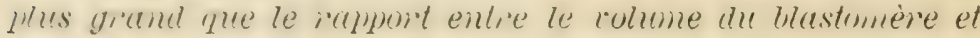
le volume de l'oent.

bans les solutions des problemes précédents, nous arons tomjour's supposé, pour plus de simplicité, que la segmentation est totale et égale et, pall suite, que les blastomères isolés sont égaux entre eux par leur rolume, et en outre, que la yuantité de substance deutoplasmatique contenue dans chaque blastomere est suflisinte pour son développement complet. Nous devons maintenant faire quelques considérations sur la chance que chacun des blastomères peut avoir d'arriver a l'itchèrement de son léreloppement, et sur l'influence que la quantité de substances dentoplasmatiques et la segmentation inégale et partiale peuvent exercer sur la possibilité de développement.

Nous avons démontré, à la solution du Ier Problème, que pour l'achèrement de la segmentation de l'oeuf', il est nécessaire et indispensable yue la quantité de substances deutoplasmatiques ne soit pas inférieure à un minimun, et que ce minimum est en rapports très étroits arec les phases le l'érolution de l'oouf piu lesyuelles les blastomères doivent pisser pour arriver a la pliase limite. P'ur conséyuent, si les phases de l'érolution sont plus ou moins nombreuses, les cellules de l'agrégat cellulaire de segmentation seront, elles aussi, plus on moins nombreuses, et le minimum de deutoplasma nécessaire pour l'achèrement le la segmentation sera plus ou moins élevé.

Or, dins le cas de l'isolement des deux premier's blastomeres, la segmentation étant supposée égale, la substance deutoplasmatique contenue dans chacun d'eux est la moitié de la quantite totale de cette substance, contenue dans l'oeuf. Mais le blastomere ", pour arriver it la phase limite 1 , doit produire 6) cellules, c'est-à-dire it peu près le nombre des cellules que prorluit lat segrnentation rle l'oeuf complet, aux dépens de la masise totale du deutoplasma. Au contraire, le blastomere c, prom arriver a la même phaso limite p, n’a qu'à produiro 33 
cellules, c'est-à-dire ì peu près la moitié du nombre normal, et cela, aux dépens d'une quantité de deutoplasina, moitié de la normale, mais érgale ì la quantité contenue dans l'autré blastomere 7 . Lequel de ces deux blastomeres aura donc plus de chance d'arriver a la phase limite? Evidemment le blastomère $c$. Nous pouvons même dire que celui-ci doit arriver sûrenent a l'achèrement de sa segmentation: car il ne doit pas produire, par ses divisions, plus de cellules que dans la sermentation de l'oeuf complet, aux dépens de lat même quantité de deutoplasma.

Au contraire, le blastomère l" derant prorluire, par ses segmentations, presqu'autant de cellules que l'oeuf complet et ne contenant que la moitié de deutoplasma, nous pourons dire qu'il arrivera probablement à l'achèrement de sa segmentation; mais nous ne pourons l'assurer comme dans le cas précédent.

Par des considérations analogues, nous pourons conclure que dans l'isolement des quatre premier's blastomères (', d, d, e, il n'y a que ce dernier dont l'acherement de la segmentation puisse être assuré : car celui-ci, bien qu'il ne contienne que $\frac{1}{4}$ de la substance deutoplasmatique, ne doit cependant produire qu'it peu près $\frac{1}{4}$ du nombre normal des cellules de segmentation, tandis que les autres trois blistoneres $(\cdot, l, d$, ne contenant, eux aussi, que $\frac{1}{1}$ de deutoplasma, et derant néanmoins produire, aux dépens de celui-ci, à peu près la moitié du nombre normal des cellules, n'ont que la probabilité d'arriver a la phase limite de l'évolution de l'oeuf.

De même, dans l'isolement les huit preniers blastomères, ou chacun deux contient $\frac{1}{8}$ de lit masse totalo deutoplasmatique, l'achèrement de lal segnentation ne peut itre assuré que pour un seul, le blastomère $y$, yqu ne doit produire qu'd 
peeu pres $\frac{1}{8}$ du nombere total des cellules de segmentation. Des 7 autres blastoneres, un, le blastomère d, doit on produire à peu près la noitié, et les 6 autres a peu près $\frac{1}{4}$ du nombre normal. Le blastomere d aura lone moins de probabilite que les autres d'arriver à acherer sa segmentation.

Pour les blastonéres $\frac{c}{2}, \frac{e}{4}, \frac{y}{8}$, l'achèrement de la segmentation est donc assuré; pour les autres blastomeres, il est seulement probable. Mais parmi ces derniers, le degré de probabilité est à peu près le même pour les blastomères $\frac{b}{2}, \frac{c}{4}, \frac{d}{4}, \frac{e}{8}, \frac{f}{8}$, parce que le rapport entre le nombre des cellules qu'ils doivent produire par leur segmentation et la quantité de substance deutoplasmatique qu'ils contiennent, est à peu près le mème; tandis que ce derrré est moindre encore dans le blastomere $\frac{d}{8}$, parce que celui-ci, ne contenant que $\frac{1}{8}$ de la masse totile du deutoplasma, doit cependant produire à peu près la moitie du nombre normal des cellules, c'est-à-dire $4 \times \frac{1}{8}$.

Toutes ces conclusions ne s'appliquent qu’a la segmentation des blastomeres, e'est-didire à la formation de la première lizrnée de cellules, lesquelles se forment aux dépens des substances deutoplasmatiques. Il nous reste donc à considérer les phases suivantes, c'est-it-dire la formation des lignées cellulaires ultérieures.

Considérons donc la deuxième lignée.

Celle-ci, nous le savons, se forme par la prolifération du blastomere " arrivé a la phase linite et des autres blastomeres qui arriveront successivement it la même phase. Mais les substances nécossaires it l'assimilation des cellules de la 2. lignée ot, par suite, at leur prolifération, sont fournies par la probiose des cellules dr la première lignée, des blastomères; et, par conséquent, la quantité de ces substiances est propor- 
tionnelle au nombre des hastonères. Inone, dans les agrégats cellulaires résultant de la sumentation des hatstomeres isolés, la quantité de ces substances sera a peu pris: uormale dans: $\frac{b}{2} ; \frac{1}{2}$ de la normale dans $\frac{c}{2}, \frac{c}{4}, \frac{d}{4}, \frac{d}{8} ; \frac{1}{4}$ de la normale dans $\frac{e}{4}, \frac{e}{8}, \frac{t}{8} ; \frac{1}{8}$ de la normale dans $\frac{g}{8}$.

Or, la deuxieme lignée cellulaire est constituée, ollo aussi, d'une série de phases dont la première est $p$, et les autres $l^{\prime}, c^{\prime}, d^{\prime} \ldots p^{\prime}$, comme nous les arons représentées pour plus de précision et pour mieux concréter nos ilées. En supposant le mème rythme de division que dans l'oeuf, nous pouvons donc déterminer que, à l'instant oi la phase p', phase limite de la 2 lignée cellulaire, sera atteinte, il y aura dans l'agrégat cellulaire au moins 62 cellules de la $2^{e}$ lignée.

On comprend d'ailleurs que si cette phase linite n'est pats atteinte, le développement te lat lignée cellulaire suivinte, dont le point de répart est dans cette phase, ne sera pas possible. D'autre part, on comprend aussi facilement que si los substances contenues dans l'agrégat cellulaire sont en quantiti inférieure au minimum nécessaire pour l'assinilation de toutes les cellules le la 2 lignée, la phase li ne pourra itre atteinte et que, par conséquent, le développement devra s'arrêter.

Lesquels des blastomeres susilits auront donc plus de chance de poursuivre leur développement ultérieur?

Evidemment, l'agrégat cellulaire du blatonere $\frac{b}{2}$, contenant dans son intérieur une quantité presque normale de substances nourrissantes, aura la plus grande prohabilité de poursuire son déreloppenent régulierement. Mais cette prohabilite serat moindre dans les atgregats dérirant des autres. blastomeres, oi lat quantité des sulustances nourrissantes sora intërieure à la mormale, et devientra très petite dans l'agrémat collulaire du blastonere $\frac{4}{8}$, oi ces substances ne seront que $\frac{1}{8}$ de la quantité normale. 
Des deux blastomeres $\frac{7}{2}, \frac{c}{2}$, le premier aura plus de probabilite de poursurre son dévoloppement que le deuxième; pour les quatre blastomeres $\frac{c}{4}, \frac{d}{4}, \frac{d}{4}, \frac{e}{4}$, le degré de probahilité sera ì peu près le mème nour les trois premiers, et moindre pour le quatriemo: et pour les huit blastomeres

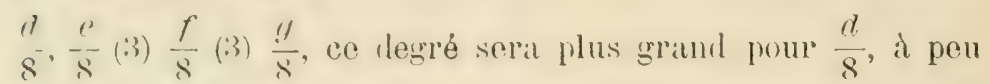
pris lo mime pour les six hlastomeres $\frac{e}{8}(3), \frac{f}{8}$ (3), et plus petit pour le blastomère $\frac{y}{8}$.

Il est d'ailleurs évident que, si nous comparons entre eux ces hlastomeres isolés a trois statles diflérents de segmentation. de l'oeuf, la probahilité de poursuirre régulierement leur déreloppement est plus grande dans les blastomères isolés au starle 2, moindre dans ceux isolés au starle 4 , et plus petite encore dans les autres isolés au stade 8.

Ce qu’il y a l'intéressint et de remarquable dans ces conclusions, c'est que les blastomères $\frac{c}{2}, \frac{e}{4}, \frac{g}{8}$, dont l'achèvement de la segmentation est assuré, ainsi que nous venons de le démontrer, sont précisément ceux-lì mêmes qui ont le moins de probahilité le poursuivre leur développement, tandis que les autres, pour lesquels la probabilité d'arriver à l'achèrement de leur segmentation est plus petite, ont, au contraire, plus de probahilité l'accomplir régrulierement leur développement, pourvu, bien entendu, qu'ils arrivent a achever leur segmentation, sans quoi, naturellement, le développement ne pourra avoir lieu ultérieurement.

Rapportons ces conclusions a des cas concrets et supposons 'fu'il s'agisse d'especes d'animax où la segmentation amene à la formation d'une blastula typiquo. Les cellules formant les parois de lat blastula appartiendront donc à la première lignée de cellules. Lat formation de la 2 lignée par l'absorption des 
substances contenues dans la cavití de la hastula amènera l'invagination caractéristique de la rastrulation, invagination qui ne pourra aroir lieu si la phase limite de l'rolution de l'oeuf, point de départ de la ?a. lignée collulaire, n'est pas atteinte.

Si la 2 lignée cellulaire, dont la formation ost la cause de l'inragination, peut ètre acherée, c'est-it-lire si sa phaso limite caractéristique est atteinte par une de ses cellules, comme cette phase est le point de lépart pour une troisième lírnée cellulaire, celle-ci se formera et amenera dans l'agrégat cellulaire une autre formation morpholonique quelle qu'elle soit, laquelle nous inliquera que le déveloprement se poursuit d'une manière normale. Mais si cette phase limite le la 2 lignée cellulaire n’est fas atteinte, la 3" lignée ne pourra se faire, et nous rerrons le développenent s'arrêter au stade de la gastrulation.

İes blastomères isolés présentent lonc, dans leur déreloppement, deux moments critiques constituant deux dificultés qu'ils doivent surmonter; mais ceux qui peurent surmonter facilement la première de ces diflicultés ont plus de peine a surmonter la seconde, tandis que ceux qui ont plus de peine it vaincre la première peurent vance la seconde plus. facilement.

Yous pourrons donc roir, dans les expériences sur l'isolement des blastomères, que, si cenx-ci sont isolés au stade de 2 blastomères, tous les deux suivent très probablement léur développenent nomal; mais si l'isolement a lieu au stade de t, de 8 , ou de lo blastomères, le déreloppement complet se fera

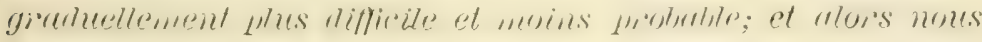

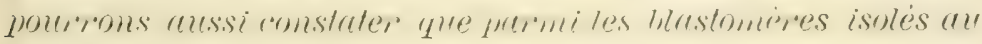
même stade, quelques-uns, - et ce seront les plus en retard dans l'achèvement de la segmentation, dont le nombre des cellules sera plus grande par rapport aur aulres - naririreront qu'd domer une blastula, et lewr développement s'ariêtera a celle phase; tandis que d'auties - et ce seront ceux 
qui ne subiront point de retard et dont le nombre des cellules de segmentation sera plus petil - ariveront jusqu'a l'achèvement de la gustrulation, mais, jaifois, ne depasseront pas ce stade de leu développement.

Ia comnaissance du rôle que la portion de substance deutoplasmatique contenue dans ehaque blastomere joue dans le dórelopenent, nous permet maintenant de juger exactement les resultats yu'on obtiendrat dans les expririences sur l'isolement des blastomères lorsque la segmentation est inégale.

Vous arons ru que le nombre des collules de segmentation dérirant de chaque blastomere n'est pas dépendant de la portion de dentophasma yu'il contient, mais de la nature bioplasmatique yu’il présente à l'instant le son isolement. Cefrendant nous venons de roir que le deutoplasma joue un role important, en fournissant au hlastonère la substance nécessaire à son assimilation et, par suite, à sa segmentation. Il ext lonc érirlent qu'une portion plus grande de deutoplasma fiacilitera beaucoup l'achèrement de la segmentation des blat stomeres qui, pour arriver a ce but, doivent produire de nomhreuses cellules, dams notre cas, fat exemple, des bastomeres $\frac{1}{2}, \frac{c}{4}, \frac{d}{4}, \frac{d}{s}, \frac{e}{8}, \frac{f}{8}$, tandis qu'une portion deutoplasmatique plus grande que $\frac{1}{2}, \frac{1}{4}, \frac{1}{8}$ de la masse totale n'exel'cera pas un action sensible sur lo dievelopement des blastomeres $\frac{r^{\circ}}{2}$, $\frac{1}{1}, \frac{!}{8}$

Mais comme le deutoplasma n'a pas d'importance pour le diveloplement do la 2 lignée collulare, colui-ci répendant de la quantité des substances sécrétées par les blastomères,

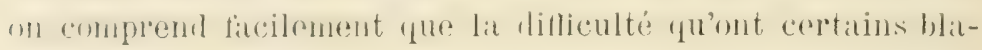
stomeres $\left(\frac{c}{2}, \frac{e}{4}, \frac{y}{8}\right)$ de poursuirre leur développement à cause de la petite quantité de ces substances, n'est pas di- 
minuée par une flus aramle quantite de deutoplasma. Aussi royons-nous que, comme l'action bienficisante les substances deutophasmatiques se horne it faciliter laacherement de la segrmentation, elle ne s'exercera que sur les blastomères qui, par leur constitution bioplasmatique, trourent des diflicultés pour surmonter ce premier stale de leur léreloppenent, ainsi que nous l'avons dimontré. Mais comme ces hlastomères sont précisément ceux mêmes qui rencontremnt le moins de difficultés dans le déreloppenent ulterieur, il est érident que le role joue par le deutoplasma dins cos expériences d'isolement est généralement assez inportant. On comprent d'ailleurs que, si l'atction bienfaisante d'une plus arande frortion de deutoplasma est déja sensible dans le cas de summentation totale et inégale, elle est d'autant plus forte lans les cas de segmentation discoïdale, où le blastomère isolé et laissé in situ, et, far conséquent, le seul qui so troure dans des conditions farorahles an déreloppenent, posside presine totalement la masso deutoplasmatique de l'oeuf.

Je m'arrête à ces considérations, parce que je crois que les Biologistes, en s'appurant sur les principes le mon interprétation, pourront eux-mênes, par de simples dérluctions losriques, résoudre tour it tour les problèmes analognues anx précédents qui, érentuellement, se présenteront daus le cours de leurs expériences. Cependant je reux, arant do laisser cette intéressante question, ajouter une rematrue, qui, peut-itre, ne seria pas sans importance prur l'explication de certains phénomenes.

Si nous comparons entre eux les agrégats cellulaires résultant de la segmentation le l'oenf complet (fir. laz) et les

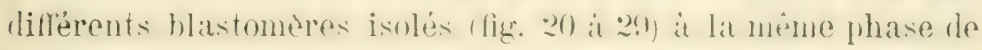

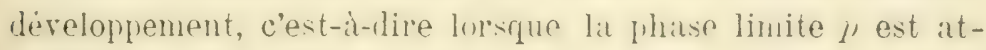
teinte par un des blastomères, nous royons que la phase la moins arancée dans l'agrenat dérivant de l'neuf complet (fig. 1:3) est $f$, tandis que dans l'agrégat lerivé de ?2, c'est $y$, (fig. 2l); 
dans ceux dérivés de $\frac{c}{2}, \frac{c}{4}$, c'est $h$ (fig. 22 et 23 ); dans ceux dérivés de $\frac{a}{t}, \frac{e}{t}, \frac{a}{8}, \frac{e}{8}$, c'est $i$ (fig. 2.2-27) et dans ceux dlérivés de $\frac{f}{8}, \frac{y}{8}$, c'est $k(f i g$. 28 et 29$)$. Nous constatons, en conclusion, qu'à mesure que l'isolement des blastomères a lieu at un stade plus arancé ho la segmentation de l'oeuf, la phase la moins arancée, repurésentée lans l'agrégat cellulatre à l'achèvement de la segmentation, est quelque peu plus rapprochée de la phase limite de l'érolution le l'oeuf. Quelle sera done la conséquence de ce fait?

supposons que, dans le léreloppement de quelques animaux, le blastomère le plus arriéré, dans notre 'cas, par' exemple, le blastomère $f$, puisse être le point de départ pour la formation d'une ligrnée cellulaire quelconque, de laquelle dérivent. plus ou moins tard, certains orwanes on certatins tissus. Il est évident que cette lignée ne pourra plus se former dans le déreloppenent des blastonères isolés: car, dans ce cals, bien que le milieu interne présente les substances nourrissantes nécessaires à sa formation, il manquera néanmoins la condition essentielle four cette formation, c'est-it-lire le blastomère qui doit itre le producteur de toute la lisnée. l'ar conséquent, l'emhryon sera privé plus tard des oryanes ou des tissus auxquels certe lignée aurait domé丶 liesu dans le déreloppement nomal. Il ne sera donc plus parfaitement complet. Mais comme ce blastomère n'influe pas sur la symétrie générale de l'embryon, celui-ci présentera toujours sa symétrie caractéristique.

J'ai jusqu'ici considéré les résultats du développement des blastomères isolés dans le cas seulement ou l'asynchronisme

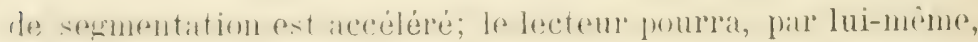
lépéter le même processus pour l'asynchronisme ralenti. Il se convaincra facilement qu'on arrive à des résultats parfaitement analogues aux précédents. 
Notons en passant, ce qui d'ailleur's ressort tout naturellement de mon interprétation le lonturénése, que le type de segmentation, c'est-it-dire lapparition de micronères et de macromeres a une phate domnée le lat segmentation, n’a qu'une importance tout-it-fait secondaire et n'influe pas absolument sur les résultats définitils du déreloppenent. La segmentation inégale et, par suite, la formation de nicromeres et de macromeres, est due a des relations physico-chinuiques qui ont lieu entre le bioplasma et les particules du deutoplasma. Elle tient donc a la constitution chimique du hioplasma, al la nature des particules deutoplatsmatiques, il la distribution de ces substances dans l'veuf, et a la direction du fusean de dirision, ainsi que je l'ai démontré dans la I Partie de ce trarail (1). Les microneres fourront donc, dans le déreloppement des blastomères isolés, itre inférieurs an nombre normal ou mème manquer completement, sans que, far ce fait, on puisse conclure d'arance à un développenent anormal de l'embryon futur.

I'ailleurs, les Biologistes pourront, l'apries mon interplétation, déterniner aussi le nombre des micrmeres, en tenant compte exactement des conditions dans lespuelles ces microméres se forment dans la segmentation normale.

Maintenant, si le lecteur lésire se convaincre le la parfinte, je rirai mème de létonnante concinlence entre ces résultats théoriques et obtemus par de simples dinluctions logiques at mathématiques de mon interprétation, at les résultats exper-

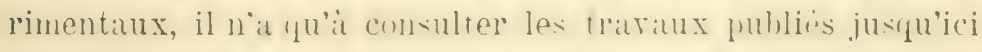
sur cet intéressant sujet. C'est naturellement arec une sa-

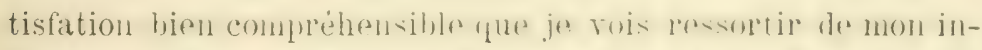

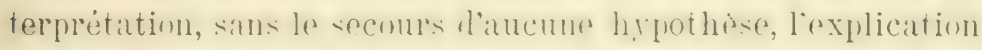
parfaite, juspue dins les details les flus minutienx, de fro-

(1) Grguro-Tos E. - Les Problemes de la Vie. 1e Partie, Chap. VII, $\S$ VII, p. 232 et suiv. 
blemes hioloriques tres complexes, dont les tentatires de solution avaient jusqu'ici échoué complètement.

Les sarants Biologistes Driescir, Morgan, Wilson, ZoJa,

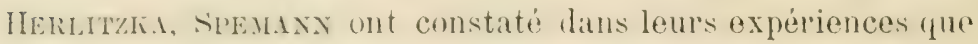
le diveloppement des blastomeres isolés aux stades : $2,4,8 \ldots$. se poursuit normalement, comme s'ils itaient remnis, et qu'il aboutit à la formation d'un embryon complet.

Inresch a expérimenti sur les oeul's d'Oursins (Echimus mi-

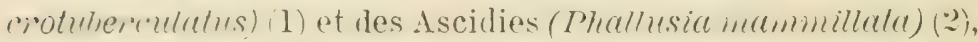
et WrLsox sur les oenfs d'Amuntoms (3). I.es expériences de ZojA ont été faites sur les oeufs des Méduses (4), avec des résultats analogues à cenx qu'araient obtenus les deux Biologistes préédents. Cepondant le travail de Zora mérite un examen spicial, à cause de certaines particularités de dréreloppement dignes de quelques considérations.

Irans le déreloppement des blastomères $\frac{1}{?}$ de rlytia flavimulu, il ohtient des embryons complets, mais plus petits que les normaux. Cependant, si nous consirérons les dimensions qu’il en donne (pag. 58 1 ), nous pourons facilement constater qu'elles ne sont pas parfaitenent la moitié de celles de l'embryon dérivant de loeuf complet, mais seulement quelque peu infirieures à celles-ci. De même, les embryons derivant des blastomeres $\frac{1}{1}$ n'ont pas des dimensions $\frac{1}{4}$ des nomales, mais a peu près noritié de celles-ci (palg. Js8). Quant au nombre des cellules constituant l'agrécrat de segmentation et les durées

(1) Drimscr H. - Entwickelungmeohanisole Studien, in : Zoitsch. f. wissons. \%ool. LIII 13d., 1891, p. 160.

(2) Drimscu II. - Ton dor Entwichelung einzolner Ascidionblastomoron, in: Arch. f. Entwickelungsmech. I Bd., 1895, p. 398.

(3) Vrrson E. B. - Amphioxus and the Mosaic Theory of Development, in: Jouru. of. Morphol. Vol. viII, 1893, p. 579.

(1) ZoJA R. - Sullo sviluppo dei blastomeri isolati dallo Hova di alcune meduse, in: Arch. f. Entwickelungsmech. Bd. I, p. 577, et Bu. II, p. 1. 
du développement, il est trés intéressant de remarquer qu'il constate que le nombre des cellules de segmentation des blitstomères $\frac{1}{2}, \frac{1}{4}, \frac{1}{8}$ est à peu près $\frac{1}{2}, \frac{1}{4}, \frac{1}{8}$ du normal, et que l'achevement de la segunentation ust en retard dans les embryons dérivés dos blastomères $\frac{1}{4}$, et plus en retard encore dans coux dérivés des blastomères $\frac{1}{8}$.

Bien que ces données, leut ére, ne soient pas parfaitement et rigoureusement précises, à cause des litłicultés qu'on rencontre dans ces expériences. par exemple, dans la numération des collules et dans le choix d'une phase fixe et constante pour tous les embryons, elles sont néanmoins suffisantes pour nous lémontrer que la coincidence mtre ces resultats experimentaux et mes résultats théoriques est remarquable.

Herlitzka, en opérant sur les oeufs des Amphibiens (1), obtient des blastomères $\frac{1}{22}$ de l'reuf de Tritom ristatus des. embryons absolument nornaux. I'lus tarr, en répétant ses expériences sur le mème animal (?), il constate que de chacun des deux premier's blastomères isolés, on ohtient des embryons entiers, ș̣métriques et, sauf la taille, parfaitement normaux; que ces embryons sont die taille plus petite que les embryons issus de l'oeuf complet, mais plus grande que la moitié de ceux-ci; enfin que les cellules de ces embryons ont leur rolume normal.

SPEMaxi 3i, en opérant, lui ausii, sur les oeufs de Tiriton, arrive à des résultats analogues.

(1) Herlitzka A. - Contributo allo studio della capaoità ovolutiva dei due primi blastomeri nell'uovo di tritone, in: Arch. fo Entwickelungsmech. B.I. II, 1896, ए. 352 .

(2) HerLutzk A. - Sullo sxiluppo di embrioni completi da blastomeri isolati di nova di tritone, in: Arch. f. Lntwickelungsmech. Bd. IV, 1897, p. 624.

(3) SPEMAxy H. - Entwickolungrphysiologische studien am Triton-Ei, in: Arch. f. Entwickelungsmech. Bd. III, 1901, p. 224. 
Mais les recherches de Infescir et de Murgax sont les plus importantes, parce quelles portent sur des particularités de déreloppement qui nous intéressent l'un manière toute spéciale.

Driescu (1) constate que la blastula, la ganstrula, le Pluteus issus d'un blastomère au statle 4 des oeufs d'Oursins, n'ont pas $\frac{1}{1}$ mais $\frac{1}{3}$ au volume normal; que le développement est plus lent à mesure que les blastomères sont isolés à une phase plus avancée de la segmentation.

Morgax, en expérimentant sur les oeufs de Fundulus (*)), obtient d'un seul des deux preniers blastomères isolés un embryon complet, plus grand que la moitié, mais plus petit que tout l'embryon normal, et, plus précisément, d'une taille i peu près égale aux $\frac{2}{3}$ de l'embryon issu de l'veuf complet.

Dans un autre travail (3), il constate que les larves d'Oursins issues d'un blastomere du stade ״ ne renferment guère que lil moitié du nombre des cellules de la larve normale; que celles provenant d'un blastomère du stade $t$ en contiennent un peu plus du quart, et que celles provenant d'un blastomere du stade $S$ en contiennent sensiblement plus du huitième.

Mais des recherches plus riłroureuses sur le développenent de l'Ambinams (1) lui permettent de constater que le nombre total des cellules présentes dans les larves dérivant des blastomeres $\frac{1}{2}$ est environ les $\frac{2}{3}$ du nombre des cellules de la

(1) Driescir H. - Die isolirten Blastomeren des Echinidentoimes, in : $\Lambda$ reh. f. Entwickelungsmech. Bd. X, 1900, p. 361 .

(2) Morgan 'T. H. - Experimontal Stucties on the Teleost Egg8, in: Auat. Anz. Bd. VIII, 1893, p. 803.

(3) Morgax 'T. H. - Sludies of the "P'trtial "Larvae of sphaereohimu, in: Arch. f. Entwickelungsmech. BH. II, 1896, p. 81.

(4) Morgan 'T. H. - The number of cells in lavere from isolated blastomeres of Amphioxus, in: Arch f. Entwickelungsmech. Bd. III, 1896, 1. 269. 
larve normale, et que la longueur de ces larves est les $\frac{2}{3}$ de ladite larve normale; que les larves provenant des blastomères $\frac{1}{1}$ montrent environ une moitié des cellules de lat larve normale et que leur longueur est moitié moindre.

Enfin Morgan, revenant tout récemment sur cette intéressante question, arrive, par des recherches plus rigoureuses, à ces conclusions: que les embryous de timenomenesles raviegatus, issus des biastomères $\frac{1}{2}$ et $\frac{1}{1}$, doml ln grestrulation

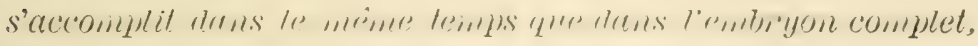
invaginent ì peu près $\frac{1}{2}$ et $\frac{1}{1}$ du nombre des cellules qu'inragine l'embryon normal; que les mbryons issus des blastomères $\frac{1}{2}, \frac{1}{4}$ et $\frac{1}{8}$, dont la gastrulation se fait plus tard que alans l'eminyon complet, invaginent un nombre de cellules proportionnellement plus grand que le normal (1).

Jo désire surtout fixer l'attention des Biologistes sul les résultats de ce dernier trarail de .InRG.Ax, parce que, ayant été déterminés arec plus de précision et de rigueur que les précédents, ils concordent d'une manière inerveilleuse avec mes résultats théoriques.

Je suis profondément conviaincu que les expériences futures confirmeront l'exactitula le mon interpretation et des solutions les problèmes précrents: mais afin que les résultats expérimentaux soient parfaitement semblables aux résultats théoriques, il est absolument nécessaire quu les expériences soient faites avec la plus gramle rigueur et precision. ()is derrat tenir un compte exact, antant yue possible, non seulement du développement d'un ou de quelques-uns, mais de toms les blasto-

(1) Morgan T. H. - The proportionalo Developmont of partial embryos, in: Arch. f. Entwickelungsmech. Bd. XIII, 1902, p. 416. 
mères isolés; du moment précis où commence lat gastrulation dans les ombryons issus des blastomères isolés, en les comparant avec un embryon issu de l'oeuf complet dont la segmentation ait commencé an mème instani que dans les oeufs dont les hlistomères ont été isolés; enfin la numération des cellules devra ètre fatite avec la plus rigoureuse précision et, ce qui est le plus important et peut être le plus diflicile, ì la même phase de développement dans tous les embryons.

\section{§III.}

\section{Sur le développement de groupes de blastomères.}

T"PRoBlève. - Déterminer les résultats du dévelomement de groupes de blastomies sépares au stade de quatre mastomères.

Solution. - La solution de ce problème est aussi simple que celles des problèmes précédents.

Les quatre blastomères étant $c, d, d, e$, les groupes do blastomères ne peuvent être que les suivants. $\left.\left.1^{\circ}\right) c d ; 2^{\circ}\right) d e$; $\left.\left.\left.3^{\circ}\right) d\left(d ; 1^{\circ}\right) c e ; 5^{\circ}\right)\left(d e: 6^{\circ}\right) d e d ; 7^{\circ}\right) c a d$.

Considérons-les séparément.

$\left.1^{\circ}\right)$ Les doux blastomeres $c$ né à $3 \mathrm{~h} .55^{\prime}$ et $d$ né à $3 \mathrm{~h} .50^{\prime}$ quoique séparés des autres blastomeres, n'en poursuivront pas moins leur segmentation. A $11 \mathrm{~h}$. 5o', un des blastomeres arrivera a la phase limite $1 \%$. La segmentation est achevée et it ce moment, l'agrégat cellulaire est constitué de jir cellules, comme il est représenté dans la fig. 30.

J'acherement de la segmentation subit done un retard. Il a lieu en mème temps que dans le développement du blastomère isole $\frac{d}{4}$; mais l'agrégat cellulaire présente un nombre 16) cellules bien supérieur à celui-ci, et quelque peu moindre

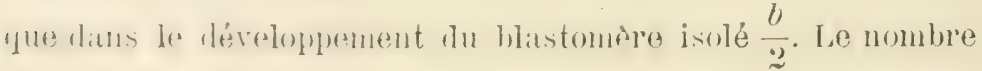


des cellules est donc moindre que le normal, mais bien supérieur à la moitié de celui-ci.

Si, au lieu de considérer le blastomère $d$ né ì $3 \mathrm{~h}$. 50 , on considere l'autre blastomère $d$ né ì $3 \mathrm{~h}$. 5a', on comprend aisément que l'agrégat cellulaire résultant sera égal à celui

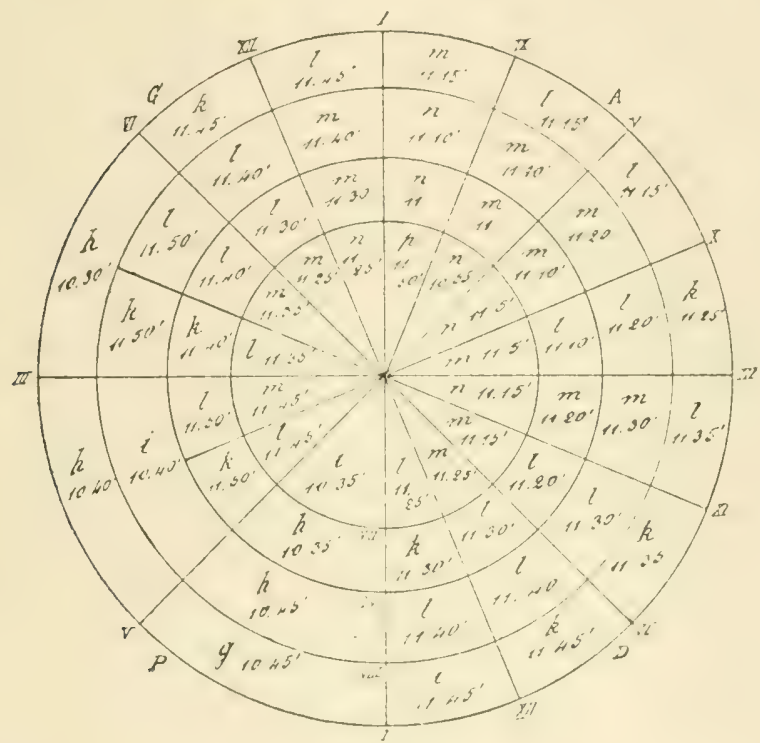

Fig. 30 ,

de la fig. 30, et que l'achèrement de la segmentation aura lieu it 11 h. 55 '.

Le développement ultérieur se fera, ici encore, comme dans l'oeuf complet, et on obtiendra la formation d'un embryon complet, quoique un peu plus petit que le normal, mais plus grand que la moitié de celui-ci.

$2^{\circ}$ La segmentation régrulière du groupe des deux blastomeres $a$ né à $3 \mathrm{~h}$. כૅว̆ et $e$ né à 3 h. 50 sera achevée à $10 \mathrm{~h}$. 30', c'est-it-dire à lat même heure que dinns le déreloppenent de l'oeuf complet ot, par suite, sans subir de retard. A ce mo- 
ment, l'agringat cellulaire (tig. :31) prossedera 3:3 cellules, c'estit-dire it peu près la moitié du nombre normal, et, précisément, le mene nombre que lans le déreloppement des blastomères isolés $\frac{c}{2}, \frac{c}{4}, \frac{d}{4}, \frac{d}{8}$. Il faut remarquer que, dans ce cas, où

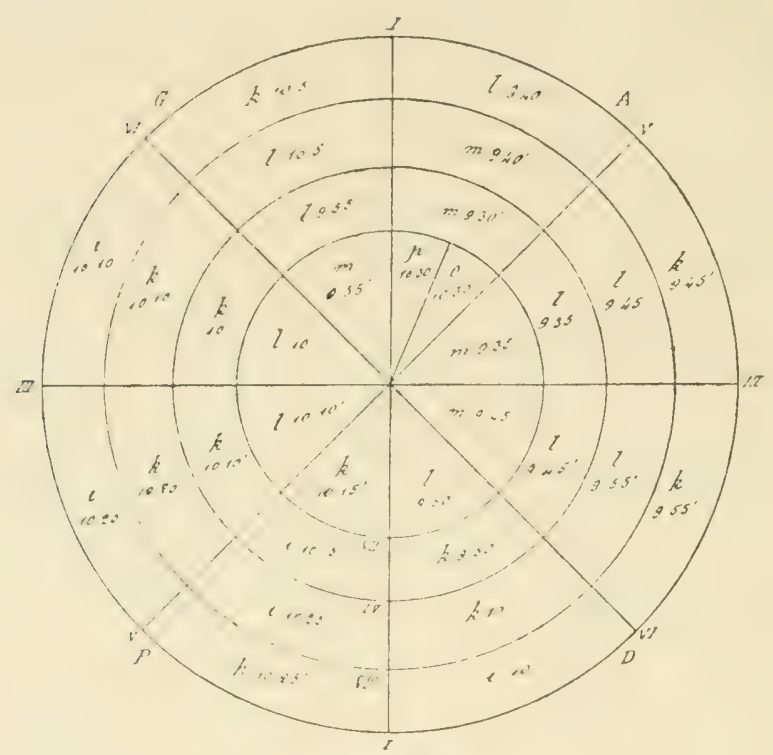

Fig. 31.

l'achèrement de la segmentation ne subit pas de retard, le rapport entre les nombres les cellules de l'agrégat de segrmentation de $\frac{d l e}{1}$ et rle l'oeuf complet est a peu près le même que le rapport entre les volumes du groupe des blastomères et de l'oeuf, c'est-ì-dire $\frac{1}{2}$.

$3^{\circ}$ ) Les deux blastomères homonymes $d$ né à $3 \mathrm{~h} .50^{\prime}$ et $d$ né à 3 h. כૅ, donneront lieu, par leur segmentation, à un agregat cellulaire tel que nous le royons représenté dins la 
fig. 3:2, ou la phase linite $\mu^{\prime}$ est atteinte i $11 \mathrm{~h}$. al', lorsque les cellules sont au nombre de ri:. L'achèvement de la seg. mentation est donc retardé, comme dans le léveloppement du blastomère isolé $\frac{d}{4}$ et du grruupe des blastomères $\frac{c d}{t}$; mais le

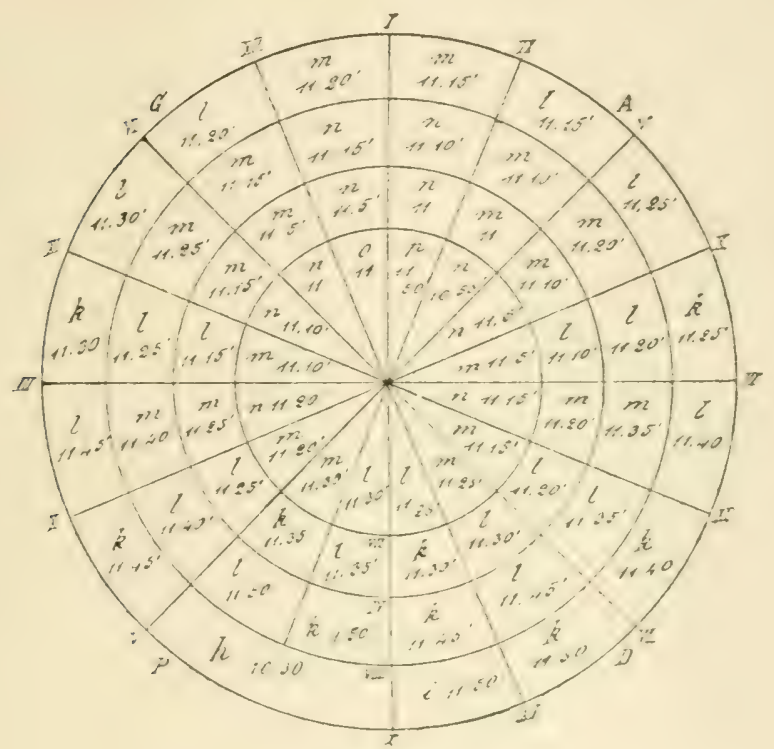

Fig. 32.

nombre des cellules non seulenent n'ent pas inférieur an nombre normal (92), mais il lui est mine quelque peu superieur (63).

Nous pourons donc arant tout arguer dès à présent que ces deux hastonè̀es aurout muins le probabilité que les autres darriver it lachèrenent le leur segmentation: car, possérlant spulement lit molié de toute la substance deutophasmatique de loeuf, ils doivent néannoins froduine un nombre de cellules jlus framl que l'veut complet. Mair ce qui mérite une consideration tout is tait farticulière. c'est lat con- 
stitution de cet incrénil cellulare, parce qu'elle nous amene it des conclusions et it des résultats inattendus sur les conséyuences possihles du développement ultérieur. Ln effet, si nous lit comparons arec les constitutions des autres agrégats collulaires, nous pourons constator une différonce sensible.

Tandis que, dans les autres agrégats, il n'y a que deux quadrants dans lesquels on puisse trourer des cellules homonymes contemporaines ou, pour plus de précision, d'une différence de sminutes soulement dans leur ìre), ici, au contraire, nous royons que toutes les cellules, d'un côté et de l'autre du premier plan de sermentation, sont homonymes et contemporaines, de sorte que cet agrégat présente une symétrie bilatérale très accentuée par rapport au premier plan de segmentation.

Quelles seront donc les conséquences qui en dériveront?

Nous savons que les cellules de la 2 lignée dérivont de la prolifération des hlastomères arrivés à la phase limite et que ces cellules forment, dans l'agrégat de segmentation, un groupe impair marquant le plan de la sýmétrie bilatérale du futur embryon. C'est donc aux dépens de ce groupe de cellules impair et médian qu'apparaitront plus tard les parties antérieures et postérieures de l'embryon.

Yous sarons encore que, bien que la 2" lignée cellulaire soit, non pas simple comme la première, mais multiple, à cause des hlastomères qui arrivent successirement it la phase limite, l'unite du groupe cellulaire ost néanmoins conservée, parce que lew blastomeres arrivant it la phase limite, sont toujours, dans l'asynchronisme accéléré, contigrus l'un à l'autre, et, par conséluent, les lignées cellulaires issues de leur prolifération sont, elles aussi, contigü's et ne forment qu'un seul groupe de cellules.

Or, dans le déreloppement de l'oeuf complet, aussi bien que des blastomères isolis ou des autres groupes de blastomeres yue: nous arons jusqu'ici considérés, il ne ponrait se former qu'un seul "roupe de cellules de la "2" lignée. C'est ce que le 
lecteur fumera constater farilement, soit en supposant dans

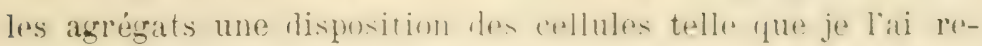
présentée dans les figures précédentes, soit encore en supposant une disposition difrirente, purru, cela est bien entromu, que cette disposition soit toujours obtenue d'après les principes du déreloppenent momolinute. l'ar conséduent, les embryons issus de ces arrezats ne peurent être que des embryous simples.

Mais dans l'agrégat cellulaire lérive du erroupe des hlastomeres: $\frac{d d}{4}$. les choses pourent se pasier hien diffiemment.

Nous royons qu'à 11 h. 50', un des blastomères, dérivé de la

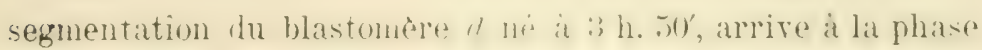
limite $p$ (fig. 32). Mais le blastomere $o$, né à 11 h. et dérirant de la segmentation du blastomère d né à $3 \mathrm{~h} .55{ }^{\prime}$, à 11 h. 55 , se transformera à son tour, sans se diviser, en $\% .111$ h. 55', et par suite, 5 minutes seulement après le blastomère $d$, né

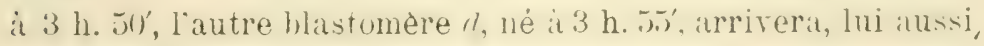
d la phase limite $p$ de l'érolution de l'oeuf.

Or, deux cas seulement sont possibles: $1^{\circ}$ ) ou les deux blastomeres arrivés d la phase limite f' et lérivant chacun alo la segmentation des deux blastumenes d sont contigus, ou hien: $2^{\circ}$ ) ils ne sont pas contigus, mais plus ou moins blogués l'un de l'autre et séparés par des cellules interposées.

Jans le premier cas, les deux lignees cellulaires promuites par la proliföration de ces blastomeres, sclont, tles alussi, contiguës et ne formeront. frar suite, yu'un seul grmpe collulaire. L'embryon qui en dérivera ulléieurement seru donc un embiyon simple, dont le plan de symélive coüncidera arec le plan d'adhésion des denix blastonières.

Jans le deuxiène car, au contraire, les deux lignées collu-

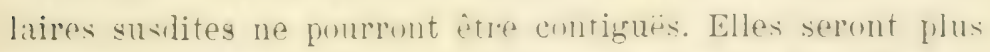

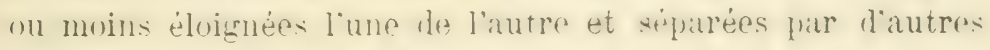
cellules, et chacune dolles regresenterat le groupe merlian impair d'un embryon. Par conséquent, dans tout l'arrégat cel- 
lulaire issu de la secmentation de $\frac{d d}{t}$, il y aura deux groupes midians impairs, separres l'un de l'autre, qui, par le déreloppement ultérieur, donneront lieu à deux embryons.

lans ce cas, on arrivera donc al ce résultat inattemdu, que le

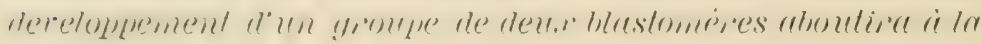
formation de deux embingons au lieu d'un seul, et que chacun de ces embryons sera à peu mès la moitie de l'embryon nm,nul. Mais comme les deux blistomeres sont adhérents, les deux entryons seront, enx-aussi, adhèrents. On obtiendra donc un embriyon double.

Quant à l'orientation réciproque de ces deux embryous, elle dépendra de la position qu’araient les deux blastomères pl dans lagrégat cellulaire. Si ces deux blastomères sont peu èloignés

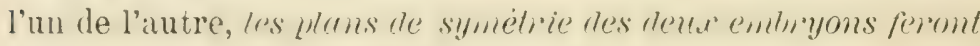
entre eux un angle petit el, par suite, une moitié d'un embryon sera plus ou moins incomplète et soudée avec la moitié contiguë de l'autre entriyon, elle aussi, plus ou moins incomnlète. si les deux blastomères sont plus éloignés, l’angle compris entre les plans de symétrie des embryons étant plus grand, la soudure des embryons s'étendra sw' une portion moins longure de lewr coins, et la valeur de cet angle pouvant ariver jusqu'a $180^{\circ}$, les plans de symétrie des deux embryons se liroureront dans ce cas sur une même droite, et la soudure des embryons se fera par les extrémités de leurs corps.

J'aroue que j'ai été moi-mème profondément surpris d'arriver à ce risultat ausi intéressant qu'inattendu. Nalheureusement, les expériences faites jusqu'à nos jours sur le déreloppement de groupes de blastomères au stade 1 sont plutrit ralres. Aussi, ne connais-je pas de résultats expérimentaux concidant arec mes résultats thérorques. Qnoi qu'il en soit, la solution que je viens de donner est une conséquence logique, mathénatique et inéritable les principes de mon interprétation. Ies experiences futures démontreront si mes résultats 
théoriques sont exacts ou inexacts; ce sera lit une preure pour ou contre mon interprétation.

En tout cas, il faudra remarquer que, ce curieux résultat ne pouvant ètre obtenu qu'ilece deux blastomères spéciaux des 1 ennstituant l'agrégat cellulaire, on ne fmurrat arriver

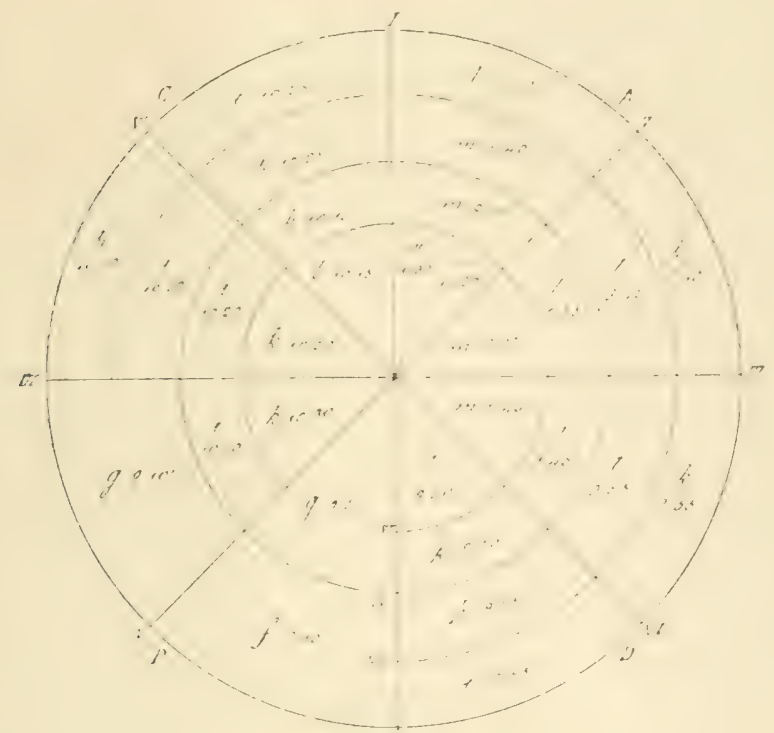

Fig. 33 .

it des résultats expérimentany concluants quen faisant de nombreuses expériences et en séparant les blastomères dans toutes les directions possibles.

$\left.4^{\circ}\right)$ Les deux blastomères c né à 3 h. $55^{\prime}$ et e né à 3 h. $50^{\prime}$, (n poursuivant leur segnentation nomale, donneront lieu a l'agregat cellulaire que f'ai représenté dans la fig. :3:3, ou lat fhase limite est atteinte it $10 \mathrm{~h}$. 33', c'est-it-dire sans retard sur le développenent ae l'oenf complet. A ce moment, le nombre des cellules est de 31). Il est donc it peine quelque peu moindre que la moitié du normal.

Il faut remarquer dans ce cas que l’agrégat cellulaire ne 
présente plus cette stmétrie hilatérale que nous pourons constater dams les autres arrigats. Mais si l'on eomsidere que derte symetrie de laterpirat de segmentation n'at pas d'importance peur la symétrio de l'embryon, ainsi que je l'at démontré au chapitre XI (par. 171), nous pourons facilement conclure

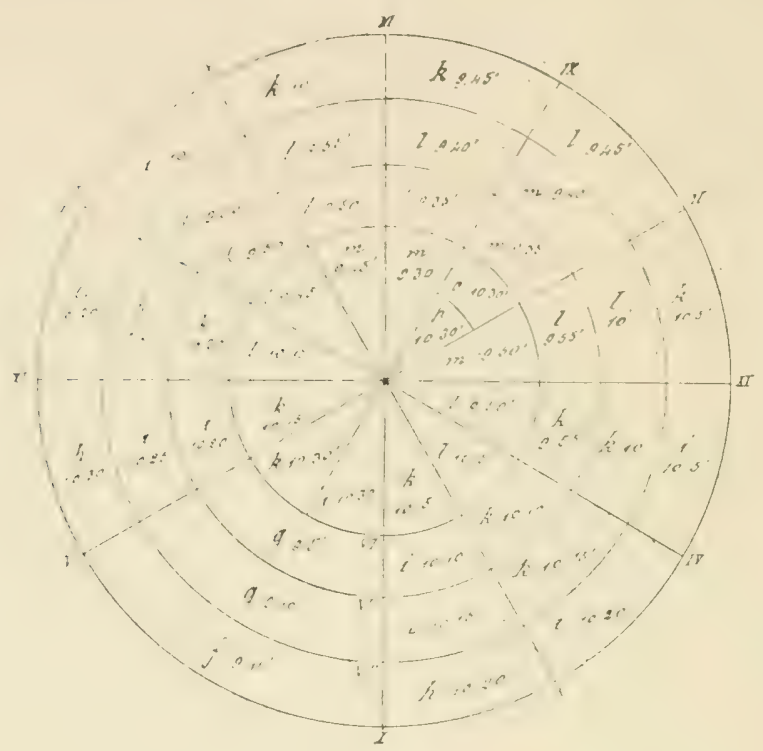

Fig. 34 .

que, dans ce cas encore, le déreloppement ulterieur domera lien it la formation d'un embry complet, quoique plus petit et à peu près la moitié du normal.

$\left.5^{0}\right)$ Les trois blastomères c né à $3 \mathrm{~h} .555^{\prime}$, d né à $3 \mathrm{~h} .50^{\prime}$,

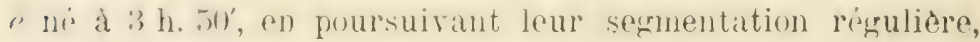
après lat réparation du blastomere d né a 3 h. 5os', donneront lieu it l'arrigat cellulate de la fig. 31 , oil la phase linite ${ }^{\prime}$ est atteinte à 10 h. 30' et, par suite, sans retard sur le développement dre l'uraf complet, et oir le nombre des cellules est de 46, c'est-à-dire à peu pres $\frac{3}{4}$ du nombre normal. 
La constitution do ent anderat on substantiellement lat

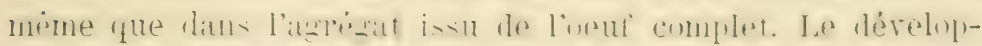
pement ultérieur donnera donc lieu à la formation d'un emhryon complet, dont le nomber des cellules sera i freu pros les $\frac{3}{4}$ du normal.

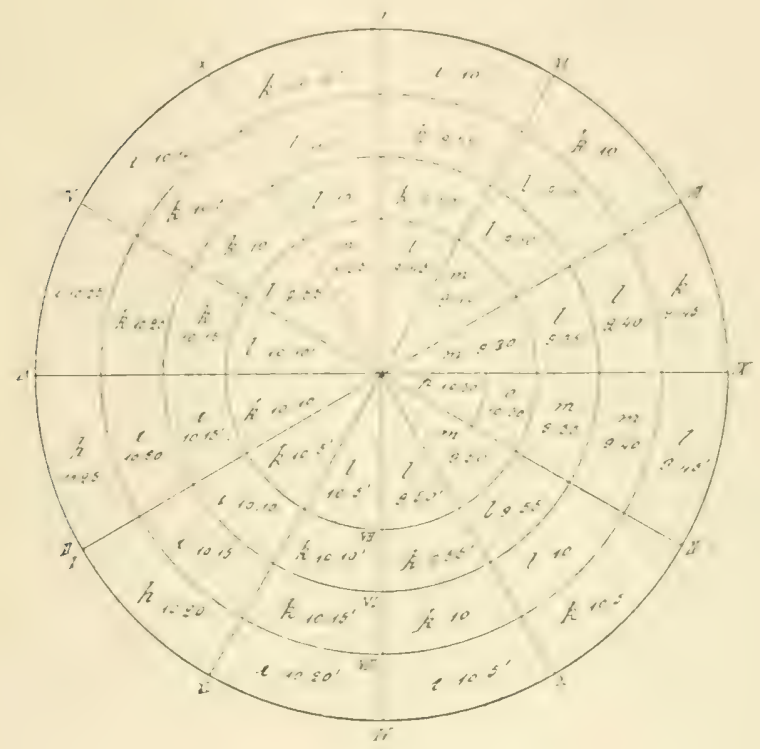

Fig: 35.

$\left.6^{\circ}\right)$ Les blastomères $d$ né à $3 \mathrm{~h} .50^{\prime}$, $l$ né à $3 \mathrm{~h}^{\circ} 55^{\prime}$, $e$ né

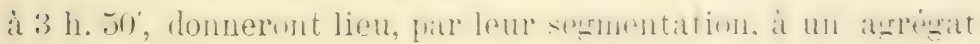
cellulaire où la phase limite sera atteinte sans retard sur le

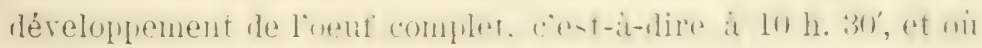

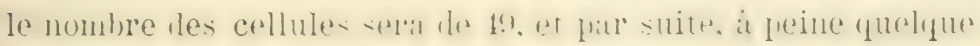
peu plus grand que les $\frac{3}{4}$ du normal.

Ici encore, l'embryon issu de cet agrégat sera complet (fig. 35). 
$\left.7^{\circ}\right)$ La segmentation des blastomères $c, d$, $d$, sera acherée a 11 h. 50', et, par suite, avec un retard de 1 h. $20^{\prime}$ sur celle he loent' complet. L'agrégat de segmentation sora constitué de a) cellules, c'est-it-dire d'un nombre de cellules a peu près une fois et demie plus grand que le normal (fig. 36).

Il est donc évident que ce groupe de blastomeres aura moins

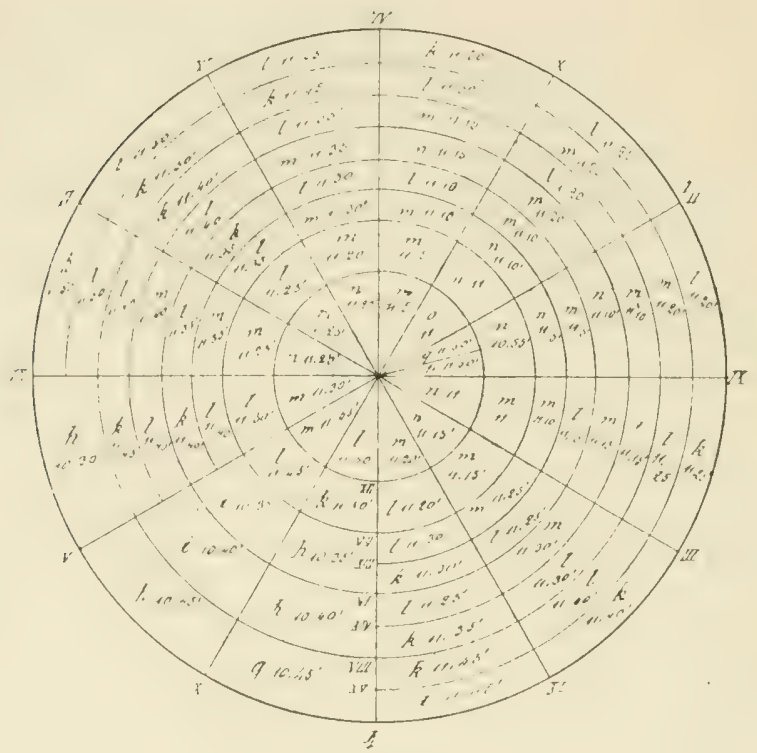

Fig. 36 .

de frobabilité que les autres d'arriver à l'achèvement de sa sergmentation, parce que, ne possélant que les $\frac{3}{4}$ de la masse totale de deutoplasma de l'oeuf; il devra produire un nombre de cellules de semmentation une fois et demie plus grand que le normal.

Mais si l'on suppose que, malgré cette pauvreté de deutoflisma, il puisse foursuive son développenent ultérieur, on derra alors appliquer a ce groupe les considérations faites 
pour le groupe $\frac{d t}{1}$ sur les risultats posibihles de développement, c'est-i-dire que, dans ce cas encore, il pourra se former ou bien un embryon seul, ou bien deux embryons soudés entre eux par une porlion plus ou moins longue de leu's corps.

En conclusion, le développenent de gpoupes de blastomères au stade 4 pourra aboutir aux résultats suivants: $1^{\circ}$ ) les groupes de deux blastoneres donneront lieu le plus sourent i la formation drun embryon seul et complet, mais parfois it la formation de deux embryons soudes jur une portion de leurs corpss; ils pourront retariej l'acherement de leur sergmentation, ou bien l'aecomplir dans le même lemps que l'oent' complet; dans les groupes ou ce retard a lieu, te nombre des cellules de segmentation el, par suile, dans une certaine mesure, le nombre des cellules des oryanes, sera ì peu près normal ou quelque pen infèrieur; dans les groupes, au contraire, oil l"on nobserve pas de retard, les cellules de segmentation seront en nombre it pert mres égal it la moitie du nommal, et le nombre des cellules des organes peubétre quelque peu supevieni a la moitie du nombre normal; zo) les groupes de trois

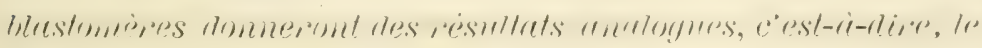
plus souvent, des embryons simples et complets, mais parfois des embryons doubles; ceux qui ne subissent pas de retard dans l'achèrement de leur segnentation domeront toujours lieu ì la formation d'un embryon simple, et le nombre des cellules de segmentation et, par suite, ie nombre des cellules des organes, sera à peu près les $\frac{3}{4}$ du nombre normal ou quelque peu supérieur aux $\frac{3}{4}$; ceux, uu contraire, qui subiront du retard dans lachèrement de leur segmentation auront moins de probabilité de se développer ultérieurement. et powront domner lieu à la fomation d'embryons douldes. Je crois pouvoir m’abstenir de donner les résultats du dé- 
veloppement des eroupes de hlastomeres separes an stade s, parce que tout Biologiste pourra les obitenir par lui-même d'après la méthode très simple que j'ai suivie dans la solution du problème précédent. Il pourra constater que les résultats seront il peu près analogues it ceux que je viens de déterminer, sauf, bien entendu, le nombre des cellules de l'agrégat de segmentation et des organes des embryons.

Les expériences sur le développement de groupes de blastomères ne sont pas nombreuses; aussi, ne pouyons-nous guère espérer y trouver une oonfirmation de tous les résultats théoriques exposés. Mais le lecteur pourra se convaincre que la coincidence entre une partie de ceux-ci et les résultats expérimentaux que nous connaissons ì présent, n'est pas moins parfaite que dans le cas de déreloppement des blastomères isolés.

Driesch, en expérimentant sur les oeufs d'Oursins (1), obtient d'un groupe de trois blastomères séparés au stade 4, un embryon complet. WiLsur arrive à des résultats semblables en opérant sur des groupes de blastomères aux stades 4 et $S$ de

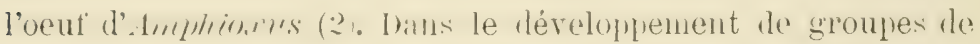
blastomeres au stade 4 , il a obtenu des embryons doubles, triples et quadruples (1. jsis); mais malheureusement, ces résultats ne peuvent être acceptés sans discussion: car l'isolement des groupes n'a pas été complet, ainsi que l'auteur mème l'avoue dans son travail.

Zosa (3), en opérant sur les veufs de Liriope mucronalu, a whtenu le développenent de groupes de denx hastonères au stade 4 et de 4 blastomères au stade 8 , aboutissant i la formation d'embryons normaux, quoique plus petits.

(1) Driescir H. - Entwichelungsmeohanischo Studien, in: Zeitschr. f. wissens. Zool. Bd. LV, 1892, p. 5.

(2) Wrison E. B. - Amphioxus, and the Moscic Theory of Development, Journ. Morph. Vol. viri, 1893, p. 579.

(3) ZoJA R. - Sullo sniluppo dei blastomeri isolati dallo nova di alcune meduse, in: Areh. f. Entwickelungsmech. Bd. II, 1896, p. 12. 
Morgan (1), en comptant les cellules d'un agréerat de segmentation dérivant de groupes de 2 blastomères au stade 4 , peut constater que le nombre de celles-ci est plus grind que la moitié du normal.

$$
\S I V \text {. }
$$

Sur le développement de portions de blastula.

VIe PRoBLine. - Déterminer les résultats du développement de portions de l'agrégal celludaire de segmentulion.

Solutron. - Considérons l'agrégat cellulaire de segmentation à l'instant où la phase limite rient d'être atteinte par un des blastomères, et supposons que cet agrégat soit fragmenté. Les fragments peuvent être, natuiellement, deux ou plus de deux, égaux ou inégaux. D’autre part, l'agrégat peut être divisé suirant plusieur's directions diffẻrenies.

Examinons ces cas séparément et supposons toujours, ce qui est bien entendu, que dans la séparation des portions de l'agrégat, le liquide de la cavité de segmentation ne se perde pas; car il est évident que lit perte totale de ce liquide emprecherait le déreloppenent ultérisur. Xons satrons d'ailleurs que dans la plupart des cals, - i l'opération de la fragmentation est exécutée soigneusement, cette perte n'a pas lieu, parce que les limbes des morcenux conpes se soudent entre eux tout naturellement.

$1^{\circ}$ ) L'agrégat de segmentation est divisé en deux portions égales, suirant le premier plan de segmentation.

Reportons-nous á l'agrégat de segmentation de la fig. 13, et supposons-le divisé suivant la ligne I-I. On obtiendra deux parties de volume égal à droite et à gauche du plan de division, et les limbes de ces parties se soudant entre eux, on

(1) Morgan ' I. II. - Studies of the « P'artial》 Larvae of Splearrechinu, in: Arch. f. Entwickolungsmoch, Bd. II, 1896, p. 101. 
aura la formation de deux blastulas, dont chacune sera la moitiẻ de l'agrégat entier.

11) Lat blastulat $\frac{1}{?}$ droite contient 33 blastomères, dont uu se tromve at la phase limite $\%$. Eridemment, ce blastomere, en puisint sia nourriture dims les substances du liquide de la blastula, donnera lieu, sans retard, à la formation de la $2^{e}$ lignée cellulaire et, par suite, à la gastrulation, qui est la conséquence directe de l'absorption de ce liquide et de la formation d"une autre lignée cellulaire. La gastrulation dans cette moitié aura donc lien a la meme heure que dans la blastula entiere.

Ici, par des considérations analogues à celles que nous arons faites sur le développement des blastomères isolés, nous purons aryuer que, les substances nourrissantes de la cavité de semmentation n'étant que la moitié de la quantité normale, le nombre des cellules se produisant aux dépens de ces substances ne pourra être, lui aussi, qu’à peu près la moitié du normal. Mais la constitution de l'agrégat cellulaire étant substantiellement la mème que dans l'agrégat entier on comprend facilement que cette blastula $\frac{1}{2}$ donnera lieu à un embryon complet, dont les cellules auront le même volume que dans l'embryon normal, mais seront en nombre ti peu près égal à la inoilié the normal.

b) La blastula $\frac{1}{2}$ gauche, ¿t l'instant où elle vient d'être séparée, c'est-i-dire it 10 h. 30, n’a que 29 blastomères; mais aucun d'eux n'a atteint la phase limite $p$. Le plus avancé se trouve à la phase intermédiaire m, qu'il a atteinte à 9 h. 55 '. La gartrulation ne pourra donc commencer tout de suite. Elle ne commencera qu'alors qu'un des blastomères de cette blastula arrivera i la phase limite $p$.

Or, le blastomère m né à 9 h. 55', après 1 h. 5', durée de sa période d'assimilation, se dirisera en $n, o$. Cette division aura lieu à $11 \mathrm{~h}$. Le blastomère $o$, après sa période d'assimilation 
dont la durée est de כ̌š, se transformera en $p$, et cela aura donc lieu it 11 h. 55 '.

A cette heure seulement, et pas arant, pourra se former la :2 lignée cellulate of enmmencer, par" suite, lat gastrulation, qui, dans ce cas, commencera 1 h. $25^{\prime}$ plus tard que dans le développement de la blastula normale et de la blastula précédente $\frac{1}{2}$ droite.

Mais pendant que le blastomère $m$, par deux divisions suc-

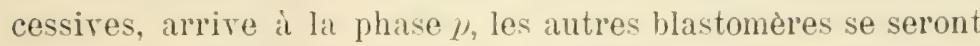
divisés, eux aussi, et ì l'instant où la phase $p$ est atteinte, le nombre des blastomeres ne sera plus de :?, mais de 60, tout comme dans le déreloppentent du hastomere isolé $\frac{b}{2}$ (fig. 21). Dans ce cas, it y aur donc un retard dans l'achèvement de la segmentation et, par suite, dans le commencement de la gastrulation; mais le nombre des cellules de segmentation sera seulement quelque peu moindire que dans la blastula entiere.

Cependant on peut, ici encore, se convaincre facilement que, la constitution de l'agrégat étant substantiellement la même que dans le cas normal, le développement ultérieur donneru lieu a un embryon complet, dont le nombre des cellules ne sera

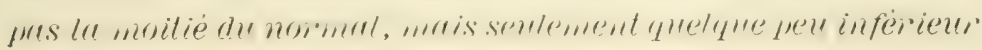
ii celui-ci.

$\left.2^{0}\right)$ L'agrégat de segmentation est divisé en deux portions égales suivant le deuxièmé plan de segmentation.

L'agrégat de la fig. 13 est divisé suirant la ligne II-II, en deux moitiés, dont l'une supérieure et l'autre inférieure.

(.) Iai blastulat $\frac{1}{2}$ supérieure contient :33 blastomères, dont wil est a lit phatse 1 . Elle commencera siuls retard lat gastrulation, tout comme dans la blastula $\frac{1}{2}$ droite (a), et l'embryou issu de son déreloppentent seral complet et possèlera à peu plès la moitié du nombre nomal de cellules. Liagregat 
ie segmentation sera constitué comme celui dérirant du déreloppement du groupe des blastomères $\frac{d e}{4}$ (fig. 31 ).

(1) La blastula $\frac{1}{2}$ inférieure ne contient que 29 cellules, tout comme la blastula $\frac{1}{2}$ gauche $(b)$. Elle ne pourra commencer tout le suite sa gastrulation, parce qu'elle ne contient aucun blastomere it la phase \%. Celle-ci ne sera atteinte qu'i 11 h. $50^{\prime}$, et, par suite, avec un retard sur l'heure normale. I ce moment, le nombre des cellules de segmentation est de 5s, tout comme dans le dexeloppenent du groupe des blastomeres $\frac{c d}{4}$ (fig. 30). L'embryon issu du développement de cette blastulit sera complet et posserdera un nombre de cellules seulement quelque peu moindre que le normal.

$\left.3^{\circ}\right)$ L'agrégat de segmentation est divisé en leux portions égales suivant le troisième plan de division.

L'agrégat de la fig. 13 est divisé suirant le cercle III. Il en résulte deux blastulas: l'une centrale, l'autre périphérique. (l)ius la réalité des faits, le troisieme plan de segmentation itant généralement horizontal, ces deux blastulas devraient itre appelées supérieure et inférieure; mais pour m'en tenir toujours anx figures données, je les appellerati centrale et périphérique).

c) La blastula $\frac{1}{2}$ centrale contient 33 blastomères, dont un ast at la phase $\%$ Mémes conclusions que pour les blastulas $\frac{1}{2}$ droite $(a)$ et $\frac{1}{2}$ supérieure $(c)$. Gastrulation sans retard; embryon complet; nombre des cellules à peu près la moitié du normal.

1) La blastula $\frac{1}{2}$ périphérique ne contient que 29 blastomirnes, dont atucun n'est a lit phase limite. Le blastomere le plus 
avancé se trouve ì la phase $m$, qu'il a atteinte à $9 \mathrm{~h} .45$. La phase limite ne sera atteinte at, par suite, la gastrulation ne

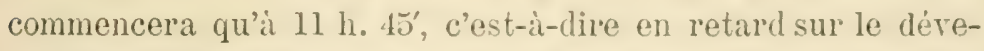
loppement de la blastula mutione. I re moment. le nomulure des blastomères sera de $\pi$. Inne dévelophement d'un embryon complet arec un nomber de cellules quelque peu mointre que le normal.

$\left.4^{\circ}\right)$ L'agrégat de segmentation est divisé en deux portions égales suivant un des plans méridiens de segmentation.

Il est érident que, quelle que soit la direction suirant laquelle l'agrémat cellulaire sera divisé en deux portions éwales, une de ces portions contienrira un hiastonere a la phase limite, et l'autre ne contiendra que des blastomères à des phises intermédiaires, dont l'un sera jus on moins rapproche de la phase limite. Par conséquent, la blastula $\frac{1}{2}$, qui contiendra le blastomère ì la phase limite à l'instant où la séparation est faite, commencera sa gastrulation d̀ l'heure normale, et l'embryon issu d'elle aura un nombre de cellules moitié du normal; l'autre blastula, au contraire, retardera plus ou moins sa gastrulation, et le nombre des cellules de sermentation sera tonjours plus grand que lat moitie du normal, quelque pen moindre que celui-ci, ef d'autant plus grand que le retard dans le commencement de la gastrulation est plus accentué.

Si maintenant mous supposons que l'agrégat de segmentation soit divisé en deux portions égales, non à l'achèvement de la segmentation, mais à un moment quelconque de sa formation et, par suite, arant que la phase limite soit atteinte par un de ses blastomères, il est érident que les résultats seront parfaitement les mêmes que les précédents. Car, dans l'une de ces portions qui contiendra le blastomère le plus arancé de tous les blastomères de la blastula entière, l'achèrement de la segmentation et, par suite, ìa gastrulation aura lieu à l'heure normale avec un nombre de cellules moitié du normal; 
dams l'autre, au contrare, l'achirement de la segmentation et la gastrulation seront plus ou moins retardés avec un nombre de cellules quelque peu moindre que le normal.

En conclusion: si lagrégul de segmentation est divisé en deua portions égales suivant une direction quelconque, toutes les deux domneront lieu it la formation d'un embryon complet, dont les cellules auront le volume normal; mais une seule de ces portions arivera it la phase de gastrulation sans retard, tandis que l'autre subira un retard plus ou moins accentue. Dans la portion où la gastrulation ì lieu sans retard, le nombre des cellules de segmentation et des organes de l'embryon sera di peu près la moitié du nommal; dans la portion, au contraire, oit la gastrulation a lien avec retard, le nombre des cellules de segmentation et des mrganes sera plus grand que la moitié du nommal, quelque preu moindre que celui-ci et drautant plus grand que le retard a été plus accentué.

Passons maintenaut ì la détemnination des résultats du déreloppement de portions de l'agrégat de segmentation lorsque celui-ci est divisé en deux portions inégales.

supposons, par exemple, que l'arrégat de segmentation de la figg. 1:3, soit divisé en deux portions inégales, dont l'une plus petite comprenant le quadrant .1, et l'autre, les trois quadrants (i I'I).

La portion $A$ contient 17 blastomeres, c'est-à-dire à peu près $\frac{1}{1}$ des blastomères de touto la blastula; mais un de ces hastomères se trouve a la phase limite et, par suite, la gastrulation dans cette bastula commencera sans retard, et l'embryon qui en dérivera sera complet et possedera un nombre de cellules à peu près $\frac{1}{4}$ du normal.

L'autre portion GPD, au contraire, contient 45 blastomères; mais aucun de coux-ci ne se trouve, à l'instant de la division, a la phase limite. Celle-ci ne sera atteinte qu'à 11 h. Jo' et l'arrégat possèlera, i ce moment, 90 blastomères, 
tout comme dans l'agrégat dérivant de $\frac{c d d}{4}$ de la fig. 36. I)ans ce cas, par des considérations analognes á celles que nous arons faites sur les résultats du déreloppenent des groupes des blastomères $\frac{d l}{4}, \frac{c d d}{4}$, nous pourons arguer que le développement ultérieur pourrat donner lieu it lat tormation ou bien d'un embryon simple, ou bien encore d'un embryon double.

Supposons maintenant que la division de l'agrégat de la fign. 13 ait lieu suivant less plans. XI-II, de manière que lat portion plus petite contiemne tous les hastoneres du qualrant $A$, et une partie des blastomères du luadrant ( $\dot{r}$, en tout 21 blastomères. Cette portion, contenant un blastomère à la phase limite, pourra commencer sil gatstrulation sans retard; et, comme elle possède ì peu près $\frac{1}{: 3}$ des hlastonires de tout l'agrégat, l'embryon qui en dérivera sera complet, mais ne possèdera qu'i peu près $\frac{1}{3}$ du nombre normal des cellules.

L'autre portion plus grande contient, au contraire, 41 blastomires, c'est-it-dire à peu pris les $\frac{2}{3}$ le tous les blastomeres le l'agrégat; mais aucun de ces hlastomères ne se trouve a la phase limite. Elle derra done retarder sa matroulation jusqu'it 11 h. 50', lorsque le hlastomere le plus arancé, qui, a 10 h. 30', se trourait encore à la phase $m$, atteindra it son tour la phase limite. Inans ce cas, le développement ultérieur donnera lieu i la formation d'un embryon complet, dont les cellules cependant seront en nombre supérieur aux $\frac{2}{3}$ du normal.

Je ne puis considérer tous les cas possibles de division le l'agrégat de segrmentation en deux portions inégales suivant les différentes directions. Il sera facile au lecteur de résoudre lui-neme tour a tonr tous les forblènes qui pourront se présenter, par la méthode très simple que j’ai suivie dans les solutions précédentes. Il pourrat tonjours constater que si 
l'uyiegal de senguentation est divisé en deux portions inégales suivant une rlivection quelcomque, it n'y a qu'une seule portion dont la gastrulation ne subisse pas de returd, et cette portion jiresente un nombre de cellules ayant arec le nomme noinal a pen mès le même rapport que la portion mème a avec la blustula entière, tandis que l'autire porlion retardera plus ou moins su yrastrulation; mais dans l'embryon issu de celle-ci, le rapport entive le nominire des cellules et le nominne nommal sera toujours plus grand que le rapport entive la portion mêne el la blastula entieive; quenfin chacune de ces poitions domnera lieu presque toujours a la formation a'un embryon simple et complet, et parfois, mais ties raiement, i la formation de rleux emmryons souclés entre exox.

Il nous reste à déterminer les résultats du développement le portions de blastula, lorspue celle-ci est divisée en plusieurs larties. Mais la létermination de ces resultats est tres facile et évidente après les solutions que je viens de domner. Quel 'fue soit le nombere des portions, quelle "gue soit lemr srandeur relatire, quelle yue soit la direction dams lapuelle la dirision a eu lieu, il est évilent qu'une portion seulennent contiendra, farmi ses blastomeres, le blastomire le plus arancé de la blatstula entière, et, pill conséfuent, cette portion seule arrirera it l'achèrement de sa segmentation et au commencement de sa gastrulation d l'heure normale et, par suite, sans retard, iandis que toutes les autres portions n'y arriveront qu'arec un retard plus ou moins fort. Mais tout Biologiste peut facilement comprendre que le léveluppenent ul trieur dans toutes ces portions de blastula, pourru que ce développement soit

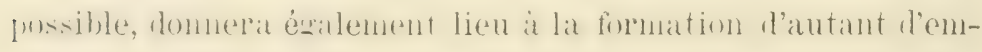
bryons complets qu'il y arait de portions.

En conclusion, si l’agrégat de segmentation est fragmenté en portions de grandeur differente et suivant des directions quelconques, chaque poition donnera lieu, par son déceloppement, le plus sourent i un embryon simple, mais complet, et paifois, mais tiès rarement, i un embryon double; mais de 
toutes ces porlions, whe seule arvivena it la gastimulation à lheure novinale et suns iretriel, el celle poition seule presentera un nomme des cellules de l'agrégat segmentution et des organes dont le rapront arec le nombre nomal sera it pen pirés égal aur rappoit entie le volume de la portion et le rolume de la blastula entière; toules les uulies portions subiiont un retard plus ou moins fort dains leur déreloppement, ot drans celles-ci, le raprort entre le nombrie de ces cellules et le nombre nomal sera toujouis plus grand que le raprort. culire le volume de chaque porlion et le volume de la thastula entière; enfin le nominge des cellules sera drautant plus grand que le retard dans le developpement est plus fort.

Par des raisonnements analogues a ceux que nous avons faits sur le développement des blastomères isolés, nous ponrons, ici encore, nous convaincre facilement que la probabilite arhever leur developpenent est moindie drus les porlions phes petites de blastula que dans les phus grandes; qu' a parilé de girandeui, les poitions qui ne subissent point de relard dans la gastrulution ont moins de mababilite de continuer leni dereloppenent que les auties portions.

Les premières expériences sur le développement des portions de blastula sont dues à HaEckel (1). Ce savant Biologiste a expérimenté sur les agrégats de segmentation de Cirystallodes, en les divisant en deux, trois et quatre portions de grandeur différente, et il a toujours obtenu des embryons normaux ou presque normaux, quoique plus petits et n'arrirant pas à leur développement complet.

DRIEScir, en opérant sur les blastulas de Sphnerectinus gramularis et de Asterias glacialis (2), a obtenu de petites gastrulas ou des formes larrales ultérieures normales.

(1) HAECLEr E. - Zur Entrichelungsgeschichts der siphonophoren, in: Natuurkund. Verhand. prov. Utrecktsch Genootschap ran Kunsten en Wotenschappen. 1869, p. 73.

(2) Driescr II. - Zur Analisis der I'otonzon embryonaler Organzellen, in: Arch. f. Entwickelungsmech. Bd. II, 1896, p. 171. 
Mordia 1) ohtient des gatstrulats normales en fragmentant

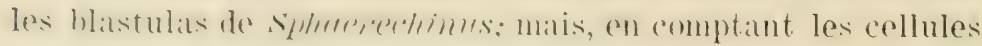
dex furtinns déreloppées, il constate que lo nombre de ces cellules est proportionnel at lat erandeur de la portion, et que les portions excisées commençaient leur gastrulation avec le mime nombre de cellules qu'elles avaient au moment de l'expérience, sans atroir le pouvoir l'arriver a en atteindre le nombre normal.

Ainsi qu'on le roit, les résultats des expériences de Morgax ne concident par parfaitement arec mes résultats théoriques, l'après lesquels il n'y a jamais qu'une seule portion dont le mombe des cellules suit proportionnel a lia grandeur de la purtion: toutes les autres portions doivent présenter un nombre de collules proportionmellement plus grand. Mais je crois que d'autres expériences, faites avec plus de rigueur et en tenant compte surtout du déreloppement de toutes les portions, sont nécessaires pour la solution définitive de cette question.

$$
\text { S } \mathrm{V} \text {. }
$$

Sur le développement d'extraovats.

On sait que Loen a fait d'intéressantes expériences sur le descroplement des oeufs d'Oursin placés dans des conditions. particulières ( 2$)$.

En portant des oeufs d'Oursin (trobacia) dans l'eau de mer diluée, 15-20 minutes après la fécondation, par suite de la difërence de presion osmotique, l'eau de mer pénetre dans loxuf, le fait gonfler et sa membrane éclate. Le protoplasma sort alors par la fissure et forme un extraoval, tandis qu'une

(1) Morgan 'I. II. - Studies of the " I'artial Darvae of Sphaerechimus, in: Arch. f. Entwickelungsmech. Bd. II, 1896, p. 107.

(2) Loes J. - Beiträge zur Entwickelungsmechanik dor aus einem Ei entstehenden Doppelbildungen, in: Arch. f. Entwickelungsmoch. Bd. I, 1895, p. 453. 
autre partie du protoplasma restant dans l'oeuf forme un intraorat. Tant que l'oeuf reste dans l'eau de mer diluée, la segmentation n'at pas lieni mais his guon le porte dans l'eatu de mer normale, la segmentation commence.

VII ProbriEme. - Quels seront les iésultats de ce développement d"après les mincipes de mon interprélation de l'ontogénèse?

Solution. - Il faut remarquer arant tout qu'après la formation de l'extraorat, tout l'seur' a perdu sa forme spherique frinitive, pour acyuérir une torne ohlongue. Lal rlirection du prenier fuseau de segmentation ne selat donc pats déterminée par l'action de la gravité et de la position réciproque des corpuscules centraux, conne je l'at démontré dans la I" partie de ce travail (pag. 251), mais bien par l'action de la forme oblongue de l'oeuf. Le premier fuseau de division se placera done dans la direction du grand axe de l'veuf, (voir: I Partie, pag. 262) et le ler plan de segmentation divisera donc l'oeuf en deux blastomères, dont l'un sera constitué par l'extraovat et l'autre par l'intraorat. C'est ce que LOEB a précisément constaté dans ses expériences.

Or, comme les deux blastomères sont adhérents entre eux, Ia direction du deuxième plan de segmentation sera déterminée par la raleur de l'adhésion (V. I e Partie, pag. 2:4), et, par suite, si la surface d'adhésion des deux blastomères est grande, il sera perpendiculaire au premier plan; si, au contraire, la surface d'adhésion est petite, il aura une direction déterminée par d'autres actions.

Mais la grandeur de la surface d'adhésion dépend de la largeur de l'ourerture de la mombrane de l'nenf par laquelle l'extraovat est sorti. Si donc cette ourerture est grande, la surface d'adhésion sera, elle aussi, grande, et le leuxième plan de division sera perpendiculaire ali premier. Dans ce cas, les deux plans s'entrecroiseront, et le troisième plan de semmentation sera, à culnse de l'arlhésion des quatre hastu- 
mères, perpendiculaire aux surfaces d'adhésion et, par suite, équatorial, tout comme dans le déreloppement normal.

Tous ces trois plans s'entrecroiseront done, et le point interne commun aux trois plans sera le point de départ pour la lomation de lat carite de semmentation, qui, par conséguent, scrat une seule. Lat sementation ultéreure accroitra le nombre des cellules; mais la cavité de segmentation sera toujours unique et à l'achèrement de la segmentation, on obtiendra nue hastulit unique, quoique ces cellules se trouvent en partie au dehor's de la membrane de l'oeuf. L'extraovat et l'intraorat n'aument rn smmme donné lien, parr leur segmentation, qu'it une bastula unique et cela, à cause de la grandeur de leur surface d'adhésion.

Il est érident que dans ce cas, le développement ultérieur s'accomplissant normalement, il ne se formera qu'un seul embryon, dont le nombre des cellules et les dimensions seront normales.

Nous pourons donc conclure que si, dans la formation des extraorats, la largeur de la fissure de la membrane par laquelle une parlie de l'oeuf fait hernie au dehor's est grande, on nobtiendra qu'une cavilé de segmentation unique, et, par. suile, une blastula unique, arec un nombre de cellules de segmentation nomal et, plus tard, un embryon unique de grendeur normale el avec un nombie de cellules normal.

Ces résultats théoriques concordent parfaitement arec les résultats axpérimentanx que LoEb at ohtenus dians certains cats (p. 462, 6), où, du déreloppement de l'extraorat et de l'intraovat, il n'a vu dériver qu'une blastula avec une cavité de segmentation unique et, plus tard, un embryon seul.

Supposons maintenant que la fissure de la membrane de loruf par langelle l'extriorat fat hernie an dehor's soit petite, et que le premier fuseau de division se place dans la même direstim quanparasunt. Le frenier plan de division séparera, lans ce cas encore, l'extraovat de l'intraovat, c'est-à-dire les deux premiers blastomères; mais la surface d'adhésion de 


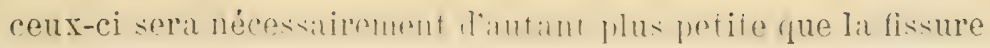
de la membrane de l'oeuf est petite. Le $2^{\circ}$ plan de division pourra bien n'être point perpendiculaire au premier, mais avoir une direction quelconque dépendint de la forme des blastomères, ou bien encore des autres actions qui influent sur la direction des fuseaux de division, ainsi que je l'ai démontré dauss la le Partie de ce travail (Chap. IX).

En tout cas, quelle que soit la direction du deuxième plan

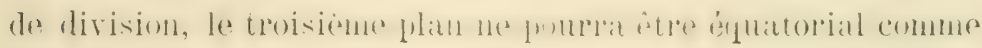
diuns la segmentation normale. Nous avons vu en eflet que si le 3e plan est équatorial, c'est que, par' suite de l'arlhésion

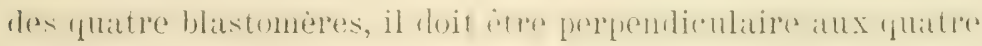
surfaces d'arhésion, lesquelles, dans le cas normal, sont égales ou presque ézales entre elles et, par conséquent, exercent sur la direction du fuseau une action de la même valeur ( $\mathrm{l}$. I Partie, p. 255). Mais comme, dans ce cas spécial, la surface d'arhésion de l'extraovat et de l'intraorat est très petite, et, que, par suite, les surfaces d'adhésion des deux blastomères de l'extraorat et de l'intraorat sont beaucoup plus grandes, l'action exercée par. l'adhésion de ces blastomères sera plus forte que celle exercée par l'adhésion de l'extraorat et de

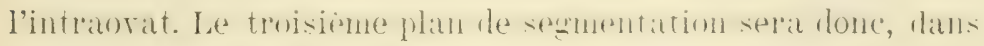
chaque partie de l'oeuf, perpendiculaire au deuxième.

L'extraorat, aussi bien que l'intraorat, présenteront donc quatre blastomèes, allérents mutre anx; et, par conséquent,

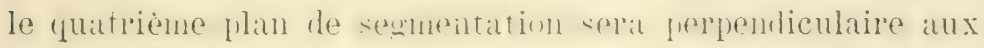
surfaces d'adhésion de ces blastomères, c'est-i-dire qu'il sera équatorial, tout comme le troisième plan l'est dans le développement normal. En peu de mots, l'intraorat et l'extraovat n'étant soudés que par une surface petite, et inférieure aux $\frac{2}{5}$ de leurs diamètres (V. I Partie, p. 22. ), et, par conséquent, leur adhésion n'exerçant pas une action suflisante sur la direction des plans dr semmentation, ils se liviseront tout comme sils étaient isolés. 
Le 4 plan de segmentation entrecroisant alors le $2^{\circ}$ et le 3" plan, déterminera done dins chacun deux la formation d'une cavite de sementation et, lit segmentation progressant normalement, on obtiendra deux hastulas, dont l'une dérivée de la semmentation de l'extratorat et l'autre, de lia semmentation de l'intriovat. Et, comme l'intraorat of l'extraorat correspondent anx deux premiers blastomeres, les deux blastulas dériveront chacume d'un des deux premiers blastomères.

I ce point, nous n'avons qu'it recourir aux solutions donneis a l'ègard du déreloppement des deux premiers blastomères isolés et it les appliquer intégralement au déreloppement de l'extriovat et de l'intraovat.

Des leux premiers hlastomères 1 , (", l'intraovat pourra itre b, et l'extriovat c, ou bien inversement. En tout cas, l'un et l'autre donneront également lieu à la formation d'un embryon complet, et, en total, à deux embryons qui resteront soudés entre eux, ou bien se sépareront plus ou moins tard. Evidemment, le rolume de ces embryons serat dépendant du volume proportionnel de l'extraorat et de l'intraovat dont ils dérivent.

Quant an nombre des cellules, il est évident qu'il ne pourra itre égal dins tous les leux, considérés au même instant. Car si, par exemple, nous supposons que l'intranvat soit le hastomère $b$, on constatera, a in instant quelconque de la segmentation, que le nombre des cellules de la blastula issue de sa segmentation sera toujours plus petit que le nombre des cellules de segmentation de lextravat (c) consiléré au même instant. C'est ce que LOEB a sourent eu l'occasion d'observer.

Dans ce cas cependant, l'achèvement de la segmentation et, par suite, la gastrulation, se fera dans l'extraovat plus tôt que dans l'intraovat; mais à l'instant ou la gastrulation va commencer, lextriovat précenterat un nombre de cellules de segmentation i pen pres moitio hu nomal, tandis que l'intrawat jrésenterit un mombre de cellules seulement quelque pen moiudre que le normal. 
Les mêmes phénomènes aturont lieu, mais d'une manière

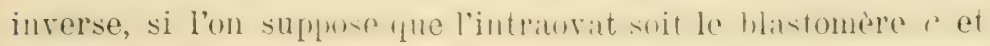
l'extraovat le blastomère 7 .

En conclusion, si, dans la formation des extraorats, la fissure de la membrane par laquelle une parlie de l'oeuf fait

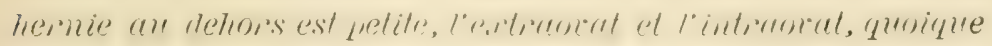
soudés entre eux, se segmenteront indépendanment comme deux blastomères isolés, et donneront lieu chacun à la formation d'une blastula et d'un embryon complet: les cellules de segmentation, a un instant donné, ne seront pas en nombre

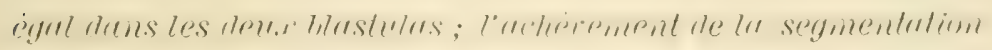
et, par suile, le commencement ie la gastinulation, aura lien plus tót dans la blastula oir le nombre des cellules est plus grand que dans l'autie; mais dans celle-ci, le nombre des cellules de segmentation, it l'instunt oir la gastrulation va commencer, sera seulement quelque peu inférieur au normal, landis que dans la première, il sera ì peu près la moitie du nombre normal.

Dans ces expériences, le développement d'un ou de deux embryons aux dépens d'un seul oeuf est dépendant de lit larreur de la fissure qui se pruluit dans la menbrane lorsque l'eau de mel diluée la fait éclater, parce que la surface d'aldhésion de l'extraorat et de l'intrarat est plus ou moins grande, suirant que la fissure est, elle aussi, plus ou moins large. Nous pourons, par suite, établir que, si le dicmétre de la surface d'adhésion de l'intraorat et de l'extraovat est au moins égal aux $\frac{\frac{2}{5}}{5}$ au dianètive de la plus grande de ces portions de l'oeuf, il ne se dévelopne qu'un embryon seul; si, au contraire, le dianètre de celle surface est inférieur ana $\frac{2}{5}$ du diamètive de la phus petite de ces poitions, chacune de celles-ci dome lieu à la fomation d'un embiyon.

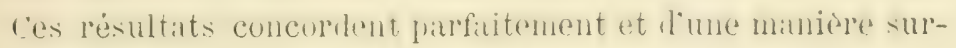
preninte arec les résultats des expériences de LoEIs. Dans ce 
as encore, non seulement mon interpretation de lontogénese nous amine a des conclusions theoriques concinant exactement arec les taits réels, mais elle nous permet aussi de honner une explication scientitique et rationnelle de ces faits intéressants, sans recourir it des hypothèses spéciales.

fe recommande aux Bioluristes lat répétition diatres expé-

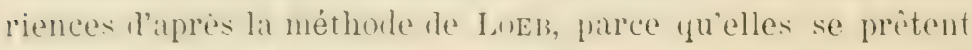
le mieux it lal vérification les résultats que j’ai déterminés, soit dans la solution de ce problème, soit dans celles des prohlènes sur le diveloppenent des blastomères isolés. Dans. res expériences en effet, les deux premier's blastomères restant somlés entre eux, nous pourons les examiner au mème instant, les comparer dats leurs phases de déreloppenent, et rérifier, latr suite, rigoureusement si, entre mes résultats thérigues et les résultats expérimentaux, il existe réellement la concordance partite que nous pourons des a présent constater seulement d'une manière générale.

\section{§ VI.}

Sur le développement de blastomères imparfaitement isolés.

Il arrive parfois, dans les expériences sur l'isolement des

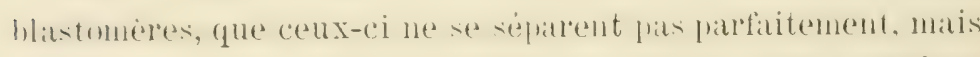
qu'ils restent plus ou moins aldhrents entre eux. Il s"igit done maintenant de:

Vili Problidie. - Déterminè les résullats du développement des blastomeres loisque ceux-ci, quelle que soit la méthorle suivie duns l'errperience, ne sont pas complètement isolés.

Solution. - Après les solutions des problèmes précédents, la solution de celui-ci est d'une très grande simplicité.

Supposons que, dans un agrégat cellulaire de segmentation, 
quel que soit le nombre de ses blastomères, on arrire, soit en le secouant, soit par d'autres moyens, à séparer les blasto-

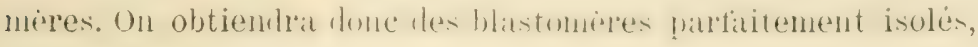
ou bien des groupes de deux ou de plusieurs blastomeres.

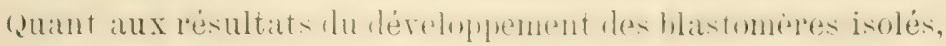
ou des groupes de blastomères, nous les trouvons dans la solution des problèmes énoncés aux \& II, III. La question qui se pose à présent est de savoir si et dans quelles conditions un groupe de deux blastomires jeut domere lien it lat formation d'un seul ou de deux embryons.

Nous arons vu que les blastomères n'exercent pas l'un su' lantre une action quelempur plas on moins nystérieuse, ou du moins que les phénomènes de l'ontogénèse peurent être expliqués, jusque dans leurs détaịls, sans recourir à l'hypothise gratuite dactions speciales. Chature hastomere suit, indépendamment des autres, la même direction dans son érolution, direction qui est léterminée par sal constitution mème. Maris la potentialité de charque blastomipe est toujours lat mème, soit qu'il se troure réuni at d'autres hlastomipers, soit quil soit parfaitement isolé.

La formation d'un ou de deux embryous aux dépens de denx bastomeres est un phenomene mécanique tres simple, dont la cause en est dans la valeur de la surface d'adhésion de ces blastomères, ainsi qu'on peut le démontrer très facilement.

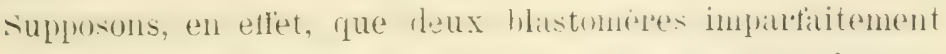
séparés adhèrent encore entre eux par une surface dont le diamètre soit au moins les $\frac{\ddot{2}}{\bar{\gamma}}$ de leur diamètre. Evidemment dans ce cas, le plan de division de ces blastomères sera per-

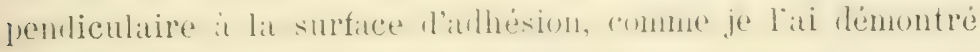
dans la I Partie de ce travail (p. 224), et le plan de division

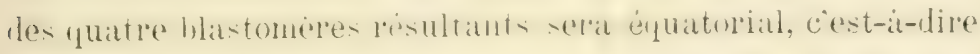
perpendiculaire au plan de division précédent et en même temps ì la surface d'athésion des deux premiers blastomèrés. 
Il se formera done une carite de segmentation unique et, parr suite, un embryon seul.

Matis, si les deux blastomeres adherent par une surface dont le diamitre est inferieur aux $\frac{2}{5}$ de leur diametre, le plan de division de ces blastomeres ne sera pas nécessairement perperuliculatre a la surfue dauhesion. Il pourra aroir une dilection quelconque dans chacun des deux blastomères.

Le plan de division suirant sera alors perpendiculaire au plan préélent et le troisibme plan sera perpendiculatre aux deux premiers. En peu de mots, les trois plans de division se produisant dans chacun des deux hastomeres sentrecroiseront, et chacun de ceux-ci se comportera dans sa segmentation comme un oeuf complet, ou comme s’il était parfatement isolé. Il se formera donc, dans chacun d'eux, une cavité de sermentation et, plus tard, un embryon et, par conséquent, .deux embryons en tout.

Il est évident que, par des raisonnements semblables, on peut litcilement lémontrer que les résultats seront analognes si, im lieu de deux, nous supposons trois ou plusieurs blastomères; par conséquent, nous pouvons conclure que, si des blastomeres sont imparfaitement isolés, ils peuvent domer. lieu ì la formation atun seul ou de phusienrs embryons; mais te résultal de leur dévelopnement n'est dû qu'i la valeur de leur. surace drathesion. Si le diametre de celle-ci est au moins les $\frac{2}{\grave{i}}$ au diamètre des blastomères, il ne se développera qu'un embryon seul (très rarement un embryon double - voir les solutions des problemes au \$III); si, au contraire, le diamètre de la surface d"adhesion des hastomères est inférienr aux $\frac{2}{5}$ du diametre de cenci-ci, il se developpera autant dembryons qu' il y a de blastomeres. 


\section{$\$$ VII.}

\section{Sur les résultats de la destruction de blastomères.}

Passons maintenant ì la détermination des résultats qu'on pent obtenir, lorsque, at une certatine phase de lat segrneshtation, on tue d'une manière quelcomque un ou plusieurs blastomères et qu'on les laisse en place.

On sait que les résultats des axpèriencess sur la destruction des blastomères ont été si discoriants qưils ont donné lieu ì de mombreusen discussions entre les biologistes. Nous verrons que tous ces résultats preurent itro expliqués d'une manière très simple et facile par mon interprétation.

Supposons arant tout le cas le plus simple. Nous passerons ensuite aux cas plus compliqués.

IX Problème. - Si l'on tue un des den p memiers blasto-

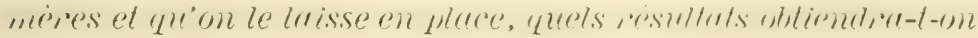
du développement de l'autre blastomère?

Solution. - Dans la solution de ce problème et des suirants, il fiut toujours supposer que les blastomeres tués sont laissés

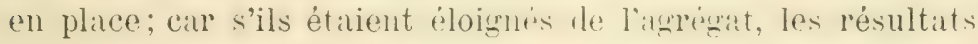
du déreloppenent lu blastomere ou des hastomeres survirants seraient ceux mêmes que nous arons déterminés aux problèmes des \$§ II, III.

Il faut en outre supposer que l'adhésion du blastomère mort arec le blastomère survirant ne diminue pas: car si elle dinninue, eomme celat peut arriver parfois, la surface d'adhésion derient plus petite, et si la valeur de son diamètre est inférieure aux $\frac{2}{5}$ du diametre du blastonteres survirant, la seg-

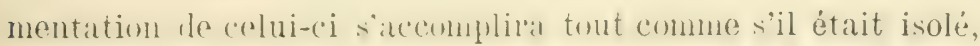
et le résultat de son déreloplement sora celui-lit mème que je viens de déterminer dans la solution du problème VIII. 
Supposons donc que, dans l'agrégat de la fig. 1, on tue un des deux premiers blistomeres, par exemple, le blastomère $b$. I) eux choses alor's sont possibles: ou bien l'oeuf' conserve sa fusition primitive et, par suite, les deux blastomeres se trouvent toujours, l'un ì droite, l'autre ì gauche du premier plan de segmentation; ou bien il subit une petite rotation et, le premier plan de sementention derenant horizontal, le blastomère tué se place inférieurement et l'autre supérieurement.

Considérons done ces deux cas et supposons arant tout que limelenserve sa position mormile. Eridemment, le blastomire $b$ ne pourra plus se segmenter, faute de son bioplasma, base de l'assimilation et de la division; mais il conservera, pendant un certain laps de temps au moins, son adhésion à l'autre blistomère.

Le blastomère $c$, au contraire, poursuirra sa segmentation régulière: car, si la destruction du blastomère $b$ à été faite sans léser le blastomère $c$, ainsi que je le suppose, il n'y a pars de raisons plausibles pour admettre que celui-ci puisse subir des perturbations quelconques. La segmentation de $c$ conduira donc à la formation d'un agrégat cellulaire, comme nous le roýons dans la fig. 13 , à droite du premier plan de segmentation I-I, c'est-ì-dire en excluant toute la portion à gauche de ce plan, dérivée de la segmentation de $b$.

La segmentation sera donc achevée à l'heure normale (10 h. $\left.30^{\prime}\right)$, et l'agrégat le segmentation présentera un nombre de cellules ì peu près la moitié du normal.

A ce point, il faut considérer la position du blastomère de lat phise limite flutr l'apport au premier plan de segmentation.

Nous avons vu que ce blastomère, dérivant de la segmentation d'un des quatre premiers blastomères, peut avoir toutes les positions possibles entre le premier et le deuxième plan de segmentation et, par suite, dans le quadrant $A$ de la fig. 13. Cette position est dépendante de la position qu'occupent successivement les blastomères qui précèdent le blastomère $p$, et dont celui-ci dérivera plus tard. Or, nous avons vu au 


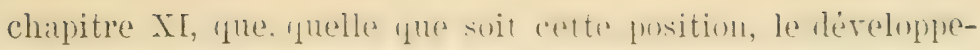
ment normal et la symétrie de l'embryon ne subissent pas lat moindre perturbation, matis que londentation de l'enbryon change par rapport au premier plan de segmentation. Car, si

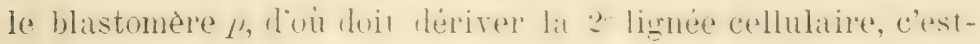
a-dire la lignée qui formera le groupe cellulaire impair représentant le plan de symétrie de l'embryon, se troure toutit-fiat contign au prenier plan de wmentation, comme nous l'avons supposé dans la fig. 13, le plan de symétrie de l'embryon coincidera presque parfaitement arec le premier plan de segmentation; si, au contraire, le blastomère $p$ se trourait contigu au deuxième plan de-segmentation, le plan de symétrie de l'embryon coinciderait arec celui-ci; si enfin il arait une position quelconque entre le $I^{\circ}$ et le II plan de segmentation, le plan de symétrie de l'embryon auriit, lui aussi, une position correspondante.

Il faut donc considérer tous ces cas possibles, parce que les résultats du développenent, comme nous le rerrons, sont étroitement dépendants de lat gusition du blastonère de la phase limite.

Supposons avant tout que le blastomère de la phase limite $p$ se troure tout-i-fait contigu au premier plan de segmen-

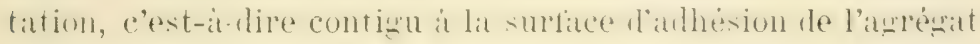
et du blastomère tué. Dans ce cas, le groupe cellulaire, con-

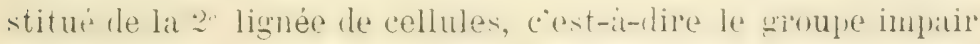
marquant le plan de symétrie de l'embryon et dérirant de la probiération du blastomere lui aussi, tout-itait contigu au premier phan de segmentation.

Mais comme nous arons ru que la symétrie bilatérale dérive de la furmation de sroupes cellubires illentiques, d'un côté et de l'autre du groupe médian impair, et comme la formation de ces groupes ne peut aroir lieu qu'aux dépens de deux blastomères identiques de chaque côté du groupe médian, ainsi que je l'ai démontré, il est érilent que si le

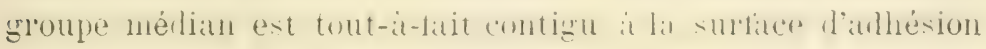


le lagrégat cellulaire au blastomere tué, entre ce groupe median et la surfice d'adhésion, il ne pourra plus se trourer ancun blastomere. l’ar conséquent, quand aural lieu la formation des groupes cellulaires pairs, déterminant la symétrie bilatérale de l'embryon dans les conditions normales, ces Eroupes ne pourront se former l'un coté et de l'autre du groupe mélian: mais il ne se formeri fu'un groupe seul, du coté du groupe médian opposé a la surface d'adhésion, daus notre exemple, du coté droit de ce groupe, le hastomère tué se trouvant à gauche de celui-ci.

Le groupe pair du côté gauche ne pourra évidemment se former, parce que, le orroupe mérlim étant supposé tout-it-fait contigu au plan d'aulhésion et, par suite, la présence de blastomères étant exclue entre ce groupe et ce plan, comme chayue lignée cellulaire a son point de départ dans un blastomère, la lignée ne pourra aroir lieu lì où les blastomères manquent.

Dans le cas que nous renons de considérer, l'embryon ne présentera donc que le groupe cellulaire médian correspondant au plan de symétrie de l'embryon et un seul des deux groupes cellulaires pairs, caractérisant sa symétrie bilatérale; et on comprend facilement que tous les organes dérivant des groupes pairs dans le dereloppement ultérieur manqueront nécessairement, dans l'embryon, du cité mème ò un des groupes pair's n'a pu se former. Dans notre exemple, l'embryon ne présentera donc, normalenent développée, que sil moitié droite, tandis qu'il sera complètement dépourvu de la moitié gauche.

Quant au groupe cellulaire médian, il sera complet, et par suite, les organes qui en dérivent seront complets, eux aussi. Mats arant supposé ce groupe immédiatement contigu à la surfare darlheion du blastonere tué, il laut tenir compte du rrila mécanique que celui-ci peut jouer dams le déreloppement ultérieur, rile dont les eflets ne peurent itre déterminés avec nne frécision riguneuse, mais que nous pourons juger d'une centaine improtance dans les phénonènes de plissement qui caractérisent la formation des organes. 
C'est ainsi, par exemple, que si lon suppose qu'ax dipens de ce groupe mélian doit se lérelopper plus tard lat grouttière médullaire, celle-ci, peut itre, ne prurrat prendre parfai-

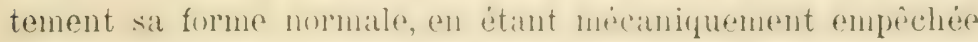
par la présence llu blastonere tué, agrissant comme obstacle.

Matis si l'on néglige ces phémonènes de malformation, qui n'ont qu'une importance tout-itfait secondaire dans notre question, nous royons que le risultat du dévelopmenent est la formation d'un demi-embryon au lieu d'un embryon entier, et que, dans ce cas, le plan de symétrie de l'embryon doil coüncider arec le premier plan de segmentation.

On comprend aisément que los cellules de ce demi-embrym auront le rolume normal. Quant it leur nombre, nous pourons le déterminer assez exactement.

En effet, conme le nombre des collules de l'atgrégit de serrmentation est à peu pres la moitie du normal, les substances contenues a l'intérieur de cet agrégat et sécrétées par les blastomères seront, elles aussi, on quantite moitié de lat normale. Le nombre des cellules du groupe impatr puisant leur nourriture dans ces substinces ne sela donc qu’i peu près la moitié du normal. Nais il n'en est pas de même pour les cellules du groupe pair. Car il est rrai que les substances nourrissantes sont, pour ces cellules ausci, réduites de moitié; mais il fiut consilerer que, dans le déreloprement nomal, les froupes pairs étant deux, ils exigent pour leur formation deux fois plus de nourriture que dans le cas que nous considérons. En peu de mots, le rapport entre la quantité des substances nourrisantes at le nombre les grmupes collukires pairs qui doivent se former est le même, aussi bien dans le déroloppement normal que dans le cas présent. En effet, si nous représentons par $/$ lit quatutitédes suhstances nourrissuntes et par 2 le nombre les groupes cellulaires pairs se formant normalement aux dépens de ces substances, le rapport en est $1: 2$; tandis que, dans le dérelupement d'un demi-embryon, la quantité des substances nourrisintes est $\frac{1}{?}$ et le nombre des 
groupes pairs est 1 . On roit clairement que le rapport est toujour's le même: $1: 2=\frac{1}{2}: 1$.

Maintenant, si l'on suppose que le blastomère tué soit $c$ au lieu de b, et si l'on suppose encore que les conditions soient les mêmes que nous arons supposées jusqu'ici, on peut, par des raisonnements analogues, arriver ì des résultats très

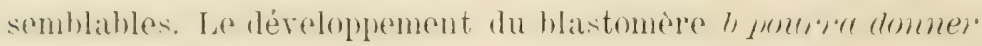
lieu i la formation d"un demi-embryon; mais, dans ce cas, le plan de symétrie de celni-ci coinncidera arec le premier plan me seffnentulion. Cependant, le déreloppenent présentera des différences que nous pouvons déterminer sur la base des solutions des problèmes précédents.

Arant tout, l'achèvement de la segmentation et, par suite, le commencement de la gastrubtion, subirat un retard (11 h. $5 \mathrm{~s}^{\prime}$ au lieu de 10 h. 30'). En second lieu, l'agrégat cellulaire de sermentation présentera it ce moment un nombre de cellules seulement quelque peu inférieur au normal.

Les substances sécrétées par les blastomères et s'accumulant a l'intérieur de l'agrégat seront donc en quantité quelque peu moindre que lat normale. Les cellules du groupe impair seront donc en nombre seulement quelque peu plus petit que normalement: mais le seul des deux groupes pairs qui s'est développé présentera évidemment un nombre de cellules plus grand que le normal.

Supposons maintenant que le blastomère à la phase $n$, au lieu de se trouver tout contigu au premier plan de segmentation, soit contigu au deuxième plan.

Il est érident que, dans ce cas, on obtiendra la formation d'un embryon entier, quoique plus petit que le normal. En aflest, lorsule, apres lat formation du groupe cellulaire impair risultant de lat : lignée de cellules, commencera la formation

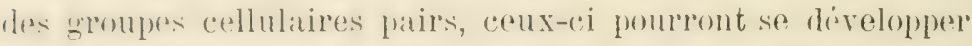
aunsi bien d'un coité que de l'antre du wroupe médian impair: car, celui-ci se trouvant placé entre des blastomeres, la for- 
mation d'un groupe cellulaire pair d'un côté sera aussitôt

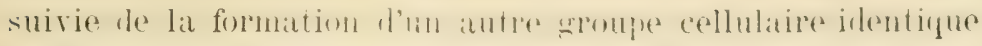
de l'autre côté. On nobliendra done jas un demi-embryon, mais un embryon entier, cest-it-dire présentant, égatement dèreloprees, les deux parlies latèrales caractérisant sa symétrie tilatérale.

Quant au nombre des cellules, celui-ci dépendra du blastomère survivant. Si le blastomere survivant est $c$, le nombre des cellules de segmentation et des organes sera á peu près la moitié du nornal; si le blastomère survivant est 1/, le nombre des cellules serat quelque pen moindre que le normal.

En un mot, le blastomère survirant domera, par son développement, des résultats analogues à ceux que nous arons déterminés dans la solution lu II problème. Je renvoie donc le lecteur i cette solution.

Quant à la direction du plan de symétrie de l'embryon, on comprend facilement que, dans ce cas, il ne pourra coincider arec le premier plan de segmentation; il coincidera, au contraire, avec le deuxième plan de segmentation et, par suite, sera perpendiculaire au premier.

Maintenant, si l'on suppose que le blastomère à la phase limite $p$ ne soit contigu ni au premier ni au deuxième plan de segmentation, mais qu'il ait une position queleonque entre ces deux plans, on comprend que l'embryon présentera également les deux parties latérales de son corps, et, par suite, qu'il ne sera pas un demi-embryon mais un embryon entier. Le plan de symétrie, dans ce cas, ne sera pas perpendiculaire au premier plan de segmentation, mais il fera avec ce plan un angle dont la valeur oscillera entre $0^{\circ}$ et $90^{\circ}$.

Nous conchurons donc que si l'on tue un des denx premiers blastomères, le développement du blastomère survivant, en supposant que l'oeuf conserve sa position nomale, poura donner des résultats diflérents, it savoir: un deni-embryon gauche ou diroit; et dans ce cas, le plan de symétrie de l'embryon coincidera arec le memier plan de segmentation; ou 
bien un emorynn entier. quoique phus petil que le normal; et dans ce cas, le plan de symetrie de l'embryon sera perpendiculaire an premier plan de segmentation, ou fera arec celui-ci un angle dont la valeur pourra osciller entre $0^{\circ}$ et $90^{\circ}$.

Il faut remarquer néammoins que, dans toutes ces déterminations, j’at fait abstraction, pour plus de simplicité, des déformations qur la frésence du hastomère, arrissant comme (h)stacle, peut profuire dans le léveloppement de l'embryon aux dépens du blastomère survivant.

Si, par exemple, le plan de symétrie de l'embryon se place perpendiculairenent au premier plan de segmentation, lal présence du blastomère tué, agissant mecaniquenent, pourra trouhler larrangenent normal des cellules de la partie antérieure ou postérieure de l'embryon qui se trouvera contiguë arec le blastomere unrt. Celle-ci ne pourra lone se lérelopper normalement; mais l'eublyon ne lerrat pas moins ètre considéré conme entier, ru qu’il présente les deux parties carractéristiques de sa symétrie bilatérale.

Si, dans d'autres cas, le plan de symétrie de l'embryon fait arec le premier plan dresementation un angle plus on moins petit, il est érident que le còté de l'embryon qui se trouvera compris entre le plan de symétrie de l'embryon et le blastomére tué ne foura pas se derelopper tout-il-fait normalement, a cause de l'empêchement qu'il trouvera dans la présence du hastomere tur; et cet empechement rerat naturellement d'aut;ut plus fort que langle compris entre le plan de symétrie et la surface d'adhèsion du blastomère est plus petit.

Dans ce cas, l'embryon pourra présenter, normalement déreluplére, me senle des deux parties latérales de son corps, taurlis que l'autre restera ingurfate; mais nous ne derrous pats moins le considérer, ici encore, comme un embryon entier.

Il nous reste maintenant ì déterminer les résultats du déreloppenent, lor-quon supuos que l'oenf ne conserve pas sa position normale. 
On sait que, dans les expériences bien connues sur la raleur,

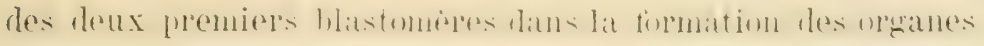
de l'embryon (1), Oscar Hertwig a souvent constaté que le bastomère tué, derenant fus lourd far rapport au blastomère survirant, se tournait en bals, de maniere que le fremier plin de segmentation et, par suite, la surficer d'alhésion des deux hitstoneres, se plaçait prespue horizntalement. Or, je crois quon doit a ce détail prostue négligrablesi Hertwa a ohtenu, dans ces expériences, des embryons entier's au lien de demiembryons, ainsi que Rovx les arait obtenus aupararant. J'espere en donner une explication satisficisante.

Supposons donc que, dans le cas de segmentation normale de l'oeuf d'un animal quelconque, les blastomères les plus arancés dans les phases de l'érolution de l'oeuf, se placent au pòle supérieur de celui-ci. (C'est ce qui arrive très probahlement dans la semmentation de loouf des dmphihiens et de plusieurs autres animaux, oì l'on roit, en effet, que la segmentation s'accélère plus an pile superpienr qu'il l’inërieur, et c'est ce que j'ai précisément supposé dans la construction rles figures (1-13) relatires a la segmentation). La cause de la position que ces blastomères les plus arancés prennent dans l'argégat cellulatre, nons échaple completenent: pent ètre est-elle tout simplement due ì la gravité, c'est-à-dire à une diffrence de densité entre les hiophatmats des deux hlitstomères dérivant le lat division du bastmmère précétent. En eflet, si l'on suppose que la densité des bioplasmas des blastomères soit de plus en plus petite à mesure que ceux-ci

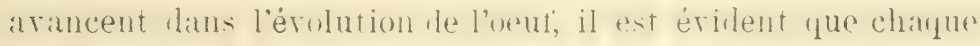
fois que le fuseau de division d'un blastomère doit, à cause

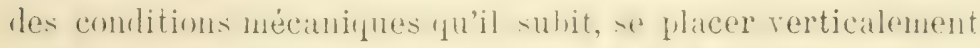
ou presule rerticalement, la hioplatuat do densité moludre st placera nécessairement mo hant of liantre, plus lourd, en has.

(1) Hertwig O. - Ueber den Werth dor ersten Furohungszellen fiir die Organbildung des Embryo; in: Areh. f. mikrosk. Anat. 42 Bd., 1893, p. 739. 
Par ce simple procédé, on explique très ficilement l'agglomération des blastomères les plus avancés dans le pôle supérieur de l'agrégart.

Mais quelle que soit lil cause de ce phénomène, ce qui n’a pas d'importance dans notre question, il est néanmoins

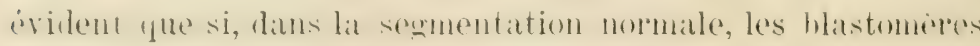
plus avancés se portent en haut, ils se porteront aussi en haut lor'que l'oeuf à changé sa position. Par conséquent, dans le cas que nous considérons, si le blastomère se tourne en bas, presque aussitòt qu'il est tué et, par suite, avant que la segmentation ait trop progressé, les blastomères plus avancés se porteront en haut, c'est-i-dire au pòle supérieur du blastomère survivant, et, par suite, ils seront les plus éloignés de la surface d'athésion du blastomère tué. Le blastomère de lir phase limite $p$ se trouvera donc, lui aussi, a cette place; il ne sera pas contigu au blastomère tué, et, comme il se troure entouré des autres blastomires, on compremal facilement que lo croupe cellulate malian qu'il formera par ses proliferations ultérieures, présenteri, d'un còté et de l'autre, des groupes cellulares pairs caractérisant la srmétrie bilatérale de l'enbryon. I)ans ce cas, on n'obtiendra done pas un demi-embryon, mais un embryon entier, plus petit que le normal, et dont le nombre des cellules sera déterminé par les solutions du II problème.

En conclusion, si après la destruction d'un des deux premiers ulastomeres, te bustomère tue se toume en bas, il y a une très giande probabilité que le développement du blastomère survivant donnera lieu, non pas it un demi-embryon, mais ì un embryon entier plus petit que le normal.

$\mathrm{X}^{\circ}$ Problìne. - Délerminer les résultats du développement lorsquion tué un des quatre premiers ulastomères.

Solution. - Il est sous-entendu que je suppose toujours que les quatre preniers blatomeres conservent leur athésion parfaite, tout comme dans le divelopuement nomal. Nous devons 
done ici considérer quatre cas difrérents, suirant que l'on tue l'un ou l'autre des quatre blastomères.

$\left.1^{0}\right)$ Dans l'agrégat cellulaire de la fig. 3, on tue le blastomère $c$.

Il est évident que les trois autres blastomères poursuirront reguliérement leur sementation, arrivant is l'athirement de celle-ci à l'heure normale $\left(10 \mathrm{~h}, 30^{\prime}\right)$ et formant un agrégat constitué comme celui de la fig. 35 dérivant du groupe des blastomères $\frac{d d e}{4}$, à cette différence près que l'un des quadrants sera occupé complètement par le blastomère tué.

Cependant, la formation du groupe cellulaire médian et des groupes pairs aura lieu comme dans le développement normal et l'embryon présentera donc sa symétrie bilatérale caractéristique. Il sera donc un embryon entier, sauf le nombre des cellules de ces organes qui sera quelque pen moindre que le normal.

Il reste à determiner l'action mécanique du blastomère tué, action qui ne peut être exactement éraluée sans des considérations sur l'origrine de la polarité de l'embryon.

J'entends par polarité de l'embryon la présence dans le corps de celui-ci d'une partie antérieure et d'une partie postérieure. Existent-elles vraiment, ces parties, au début de la formation de l'organisme? Et si elles n'existent pas, comment a lieu leur formation dans le déreloppement progressif?

On sait que, d'après mon interprétation, la symétrie bilatérale s'établit par la formation d'un groupe cellulaire médian impair et de deux groupes cellulaires pairs d'un côté et de l'autre du groupe médian. Négligeons pour le moment ces groupes et portons notre attention sur le groupe cellulaire médian.

bividemment, ce mpoupe etant constitue de cellules dérivis elles aussi, tout comme les blastomères, du développement monodique, présentera une constitution analogue à celle de l'agrégat cellulaire de sogmentation, a cette dillèrence près 
que, tamdis yue, dans l'uente, les hatatomires forment par leur

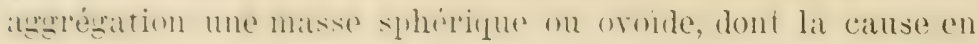

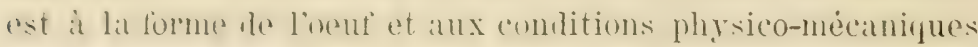
de lat segunentation, dans le wrouge cellubire modian de la $\because$ lignée de cellules, celles-ci ne se disposeront pas en forme spheidigue, mais en série linéaire lommant, dans l'arrégat de sementation, ontre les hastomères qui les environnent, une lame cellulaire, dont la forme pourra itre très différente et déterminée par une foule de causes que nous ne pourons commatre et qui, d'alleurs, n'ont pats d'importance dans notre question.

Mats quelle que soit lit forme de cette lame cellulaire logrée entre les autres blastomères et, plus tard, entre les deux groupes cellulaires pair's, il est cependant évilent que sa constitution ne sera pas égale dans toute sa longueur.

De mème que, dans la segmentation de l'oeuf, nous royons qu’i cause du développenent nonodiques, l’agregat cellulare acyulert une polarité très listincte, caracterisée far la présence à l'un de ses pòles des blastomères les plus arancés dams l'érolution de l'oeuf et, dans l'autre, des blastomères les moins arancés, le mêne aussi, dans le groupe cellulaire inpair, on constatera toujours, à cause du déreloppement momolique, une polarite très distincte, lérivant le lat mésence, à l'une les extrénités le ce groupe, der cellules les plus arancées lank lérolution caractéristique de la : lignée cellulane et, a l'atutre pxtrénité, des cellules les moins arancées daus cette même érolution. C'est d'ailleurs ce qui arrivera anssi dans la formation des groupes cellulaires pairs.

or, l'origine de la polarité de l'embryon tient précisément

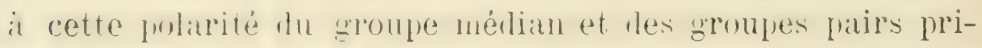
mitils. en cressus que, si mus supposons, par exemple, que les cellules lex lulus arancées le cos groupe médian puissent domer liesu, par lente érolution ultirienre, it un organe quelconque caractérisant la rérion antérienure de l'embryon, les autres

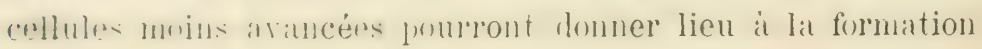




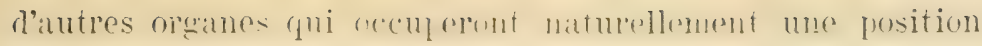
postérieure par rapport au premier, et caractériseront par suite, la région postérieure de l'embryon.

Les deux régions antérieure et postérieure du corps ne sont done pas indépendantes l'une de l'autre; elles ne peuvent, par suite, exister indépruliument, lauce que l’une delles n'existe

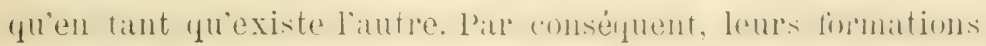
soat très étroitement liẻes, et no peurent avoir lieu indépen-

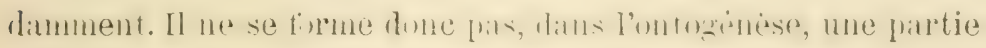

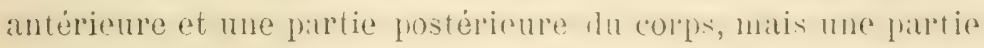

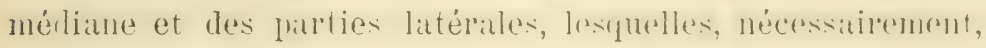

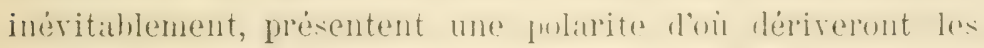
régions antérieure et postérieure. Tout cela éridemment, à cause du déreloppement monodique que nous arons posé comme base de notre interprétation de l'ontogénèse.

Cète dépendance très étroite entre ces deux régions nous explique parfaitement pourquoi, dans les expériences qu'on a

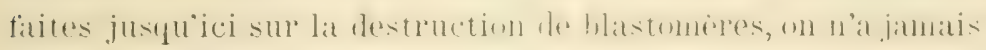
obtenu des demi-embryons autéldeurs et postérieurs. Il est rrai que Roux a obtenu, comme nous le verrons plus tard, un demi-embryon qu'il a appelé demi-embroon antérieur (Hemiembryo anterion); mais on peut facilement constater, par l'examen des figures annexées à son travail, qu'il ne sagrit pas d'un demi-embryon, mais d'un embryon entier, quoique sa parlie postérieme ne paraisse pats complistruent dérelonpée, a cause de la présence du blastomère tué, agissant comme à obstacle mécanique.

Je crois que, n'y eut-il pas d'autres motifs pour exclure toute

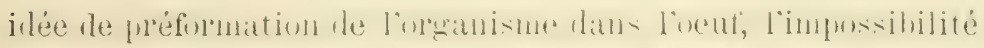

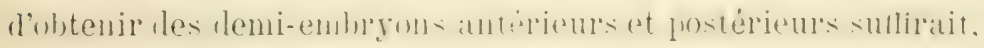
à elle seule, pour nous convaincre de l'inexactitude des interprétations ontogénétiques basées sur ce principe.

Cela étant posé, revenons maintenant à la solution de notre problème.

Aux dépens des trois blastomères surrirants se formeral 
Wone lembryon d'une maniope fout-it-lait normale, ot, par suite, arec ses deux parties antérieure et postérieure, et ses deux parties latérales. Le déreloppentent se poursuirat régulierement, et le sroupe cellulate median, dont la direction détermine la direction du plan de symétrie de l'embryon, aura, dans l'arrégat de segmentation, une position quelconque dépendant de la position qu'arait le prenier blastomère arrivé a la phase limite de l'évolution de l'oeuf.

Mais quelle que soit cette position, ou hien le groupe cellulaire médian concidera avec le premier plan de segmentation, ou bien il lui sera perpendiculaire, ou bien encore il aura une direction intermédiaire, faisant avec ce plan un angle d'une valeur oscillant entre $0^{\circ}$ et $90^{\circ}$.

Considérons ces cas différents.

A) Le groupe cellulaire médian et, par suite, le plan de symétrie de l'embryon, coincident avec le premier plan de segmentation.

Il faut considérer deux conditions possibles: a) ou bien la partie du groupe médian corresponlant à la régrion antérieure du corps de lembryon est dirigée rers le hlastomère tué, ou bien $a^{\prime}$ ) elle est dirigée en sens contraire.

(1) Lat tite de l'embryon est dirirgée vers le blastomère tué.

Dans ce cas, l'embryon se formera tout entier, arec ses deux parties littrales; mais l'accroissenent de la partie antérieure sera troublé frar l'obstacle mécanique du blastomere tuc, tandis que la partie postérieure fourras se diveloper normalement. L'embryon sera donc complet, mais présentera des déformations dans la région antérieure de son corps.

a') La tête de l'embryon est dirigée en sens contraire au blastomère tié.

Dans ce cas, pour des raisons analogues aux précédentes, ce sera la partie postérieure du corps qui présentera des détormations.

$B$ ) Le plan de symétrie de l'embryon est perpendiculaire au premier plan de segmentation. 
i) La tête de l'embryon est dirigée rers le blastomèrè tué. Mềmes résultats que dans le cas A) a).

b) La tête de l'embryon est dirigée en sens contraire au blastomère tué. Mêmes résultats qu'A) $a^{\prime}$ ).

C) Le plan de symétrie de l'embryon fait avec le premier plan de segmentation un angle dont la valeur est comprise entre $0^{\circ}$ et $90^{\circ}$.

c) La tête de l'embryon est tournée ver's le blastomère tué.

Il faut remarquer que, dans ce cas, le blastomère tué, s'oppowant mécaniquenent à l'accroissonent ultérieur du groupe collulare médian, pourrar foreror les cellules qui le forment is se séparer en deux parties, et, par suite, le groupe médian jrésentera antérieurement uno hifurcation, dont les linites seront celles mêmes du blastomère tué. On obtiendra donc un embryon arec la régim posterieum simple et la région anterieure double.

c') La tête de l'embryon est tournée en sens contraire au blastomère tué.

Conclusions analognes anx précédentes, matis l'enhryon présentera la région postirieure de son corps double et lat région antérieure simple.

$\left.2^{\circ}\right)$ Dans l'agrégat de la fig. 3 , on tue le blastomère $d$ du quadrant $G$.

Lal segrmentation ultérieure des blalstomeres " / ", se firisint normalement, donnera lieu it la formation l'un agrégat cellulaire constitué comme celui de la fig. 31 dérivant de la seg. mentation du groupe hastomerique $\frac{c d e}{1}$, siuf, hien entenlu, lat disposition quelque peu dillérente des halstomères due it lia présence du blastomere tué. Acherenent de lat sexpmentation it l'heure normale.

Ici, il faut considérer les cas suirants:

A) Le plan de symétrie de l'embryon coïncide avec le premier plan de segmentation.

Nous sarons qu'il y at concidence entre le plan de symétrie 


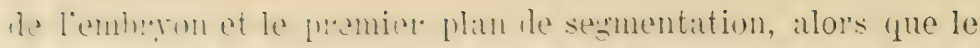
hatemene de lit phiture linite, suluche des cellules du groupe médian, est tout-í-fait contigu ì ce plan, et nous arons vu, à Iy solution du probleme précédent. que, dans ce cas, le dérelop-

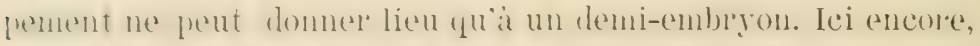
on 11 ibriemdrat donce que lat formation d'un demi-embryon, c'estit-lire d'un embryon manquant de sa moitié gauche.

Milis comme le blastomère $c$ du quadrant $I$ ' est survirant, il aurat froduit, par sa segmentation, de nombreuses cellules, lesplemes constitueront par lenr ensemble nue partie du corps le l'emblyon. Celui-ci seria done senlement un demi-embryon au proint le rue morphologique, mais il selal les $-\frac{3}{1}$ de l'embryon entier au point de vue de la masse de son corps.

Il pourra même arriver parfois qu'aux dépens des cellules he segmentation alublastomere ", il se déreloppe un embryon entiel' plus petit que le normal et plus en retard que celui-ci, restant naturellement soudé avec l'autre demi-embryon.

B) Le plan de symétrie de l'embryon est perpendiculaire an premier plan de segmentation.

Dans ce cas, la partie antérieure de l'embryon pourra être tournée vers le blastomère tué ou en sens contraire.

7) La tête de l'embryon est tom'née rers le premier plan de segmentation.

Mèmes résultats que dans le cas $1^{\circ}$ ) A) (1).

b) La tête de l'embryon est tournée en sens contraire ¿ul blastomère tué.

Nêmes résultats (qu’à $1^{\circ}$ ) A) $\left.a^{\prime}\right)$.

C) Le plan de symétrie de l'embryon fait avec le premier

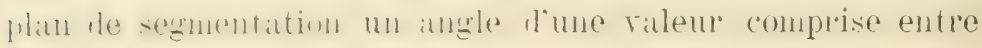
(1) et $90^{\circ}$.

c) La tête de l'embryon est tournée ver's le blastomère tué. Memes l'ésultats qu'a $1^{\circ}$ ) C) c).

$\left.c^{\prime}\right)$ La tèto de l'embryon est tournée en sens contraire au. blastomère tué. 
Mêmes résultats qu'ia $1^{\circ}$ ) C) $c^{\prime}$ ).

$3^{\circ}$ ) Dans l'agleégat de la fig. 3, on tue le blastomere $a d u$ quadrant $D$.

A) Le plan de symétrie de l'embryon coincide avec le premier plan de segmentation.

Mêmes résultats que dans 2") $B$ ) 'll et $U^{\prime}$ ).

$B$ ) Le plan de symétrie de l'embryon est perpendiculaire au premier plan de segmentation.

Iêmes résultats qu'á $2^{\circ}$ ) A).

C) Le plan de symétrie de l'embryon fait avec le premier plan de segmentation un angle entre $0^{\circ}$ et $90^{\circ}$.

Mêmes résultats qu'ì $2^{\circ}$ ) C) cet et $c^{\prime}$ ).

$\left.4^{0}\right)$ Dans l'agrégat cellulaire de la fig. 3 , on tue le blastomère $e$.

Dans ce cas, il n'y a qu'un seul l'ésultat, c'est-à-dire la formation de deux emhryons, l'un et l'autre de la mène grandeur et soudés entre eux, ou bien frar lit partie antérieure de leur corps, ou bien par la partie postérieure.

En effet, comme le blastomère $e$ est tué, le blastomère $p$ it la phase limite de l'évolution de l'oeuf ne pourra se former dans le quadrant $A$. Le premier blastomère $p$ paraîtra donc dans le quadrant $D$ à 11 h. $50^{\prime}$ et, par suite, arec retard sur le développement normal; mais 5 minutes après, un autre

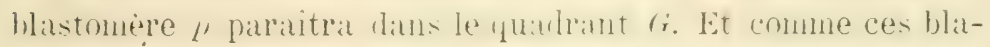
stomères sont séparés l'un le lautre par le hlistonùre tué é interposé, il s'ensuit que les grompes cellulaires médians qui en dériveront seront, eux aussi, nécessairenent séparés. Iön. la formation de deux embryons.

Mais si les deux blastomènes as trourent tout-it-fit con-

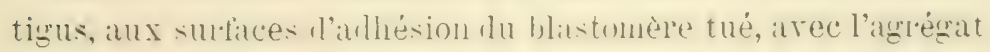
cellulaile, il s'ensuiväa naturellenent que chacun de ces deux embryons ne purrat présenter, momalement direlopree, qur la moitié latérale de son corps qui est opposée à la surface

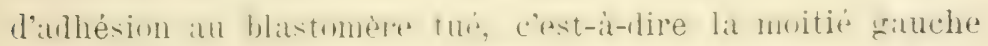

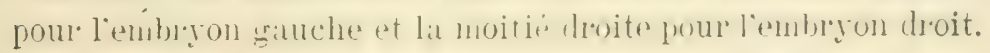


livilenment, cette séparation des deux moitiés ne pourral Sinserrer que le long des surfitces d'adhésion du blastomere tué, agrissant comme obstacle mécanique. Matis daus la portion he lagrézat oi le hastomère thé ne peut agir mécaniquement, - c'est-it-dire dans le quadrant $P$, les deux moitiés seront soudées entre elles et ne formeront qu'un embryon seul.

Dims le cas où les plans de symétrie des deux embryons coincident arec les deux premier's plans de segmentation, il se formear donc deux demi-mmbryons somlés entre eux le long l'une portion de leur corps.

si l'on suppose, au contraire, que les blastomères p' paraissant presque en même temps dans les deux quadıants $D, G$, he solent pas contigus aux surfaces d'alhésion du blastomere tué, les deux groupes cellulaires bilatéraux aux côtés du wnoupe mélian pourront se former librenent, et on ohtiendra alors lat formation de deux embryons morphologiquement entiers, soudés entre eux par une portion plus ou moins longue de leur corps.

Ein lésumé, nous pouvons donc conclure que, si l'on tue un des quatre premiers blustomeres, on peut obtenir du dereloppement des autres blastomères survivants les résultats suivants: ou bien un embryon seul morphologiquement demi (de masse $\frac{3}{4}$ de l'embryon entier), et, dans ce cas, le plan de symétrie de l'embryon cöncide avec un des deux memiers ulans de sermentation; ou bien un embryon seul morphologiquement entier (de masse $\frac{3}{4}$ de l'embryon entier) et, dans ce cas, le plan de symétrie de l'embryon ne coüncide pas arec un des deux premiers plans de segmentation; ou bien un entrigon morphologiquement entier, dont la région antèrieure ou josterieure du carps est bifurquée; ou bien, denx embryons moryholoriquement demis ot soudes entie eux; ou bien encore, deux embryons morphologiquement entiers, quoique plus petits que les normanix el soulés entre eux. 


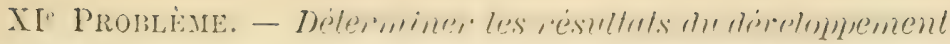

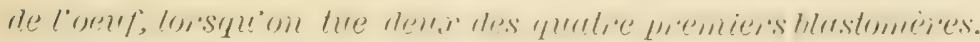

Solutiox. - Les quatre premier's blastomères (fig. 3) formant les quatre quadrants d'une sphère, on peut tuer deux blatstomères contigus ou deux blastonères opposés. ('onsilérons séparément les diffẻrents cas possibles.

1) Dans l'agrégat de la fig. 3, on tue les deux blastomères contigus $c, d$.

Les deux blastomères survivints donneront évilemment lieu, par leur déreloppement, aux mimes résultati que le seul blatstomire c, lor'squ'on the l'un des denx premier's blastomeres, c'est-i-dire à un demi-embryon, si le plan de symétrie de l'enbryon concile arec le premier plin de sermentation; ;u à un embryon morphologiquement entier, si le plan de symétrie ne coincide pas arec le premier plan de segmentation (V. la solution du problème IX).

$\left.2^{\circ}\right)$ Dans l'agrégat de la fig. 3 , on tue les deux blastomères contigus $a, e$.

Mèmes résultats que si l'on tue le blastomère $c$ á la phase de deux blastomères (V. la solution du problème IX).

$\left.3^{\circ}\right)$ Dans l'agrégat susdit, on tue les blastomères $c, c$, du même côté du deuxième plan de segmentation.

Les résultats du déreloppement des deux blastomères survivants dépendront de la position du blastomere $p$ de la phase

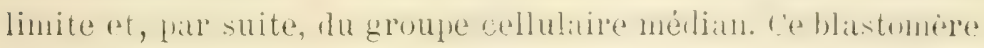
est-il tout-ir-fitit contipu au deuxieme platn de segmentation? On obtiembar un embryon morphohgripuenent demi, dont le plan

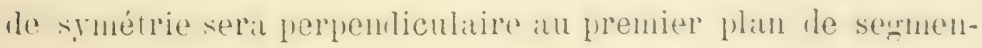
tation. Le blastomère $p$ n'est-il pas contigu au deuxième plan de sermentation? On obtiemliat alor's un embryon morpholo-

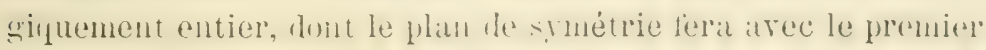

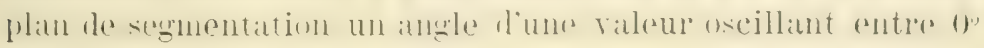
et $90^{\circ}$.

$\left.4^{\circ}\right)$ Dan's l'agrégat susdit, on tue les blastomeres contigus $d, e, d u$ même còté du deuxième plan de segmentation. 
Résultats analogues aux précédents.

$\left.5^{\circ}\right)$ On tue les deux blastomères opposés $d d$.

si lon suppose toujours que l'athexion des blastomères tués ne diminue pas et, par suite, que leur surface d'athésion aux hilstomeress survivants conserve sil valeur primitive, chacun des demx blastomeres donnerat lieu it la formation d'un embryon morpholoniquement demi, si le plan de symétrie de l'embryon comede aree un des deux premiers plans de segmentation, on bien it un embryon entier, si le plan le strmétrie ne coincide pas arec un des deux premiers plans de segmentation (V. la solution du prohlème IIL", sur le déreloppenent des quatre premiers blastomères isolés).

$\left.6^{\circ}\right)$ On tue les deux blastomères opposés $c e$.

Mèmes résultats que dans le cas précérlent ( $\mathrm{V}$. la solution du problème $\mathrm{II}^{\mathrm{e}}$ ).

En conclusion, si l:on tue deux des quatre premiers blastomères, on obtiendra du développement des blastomères sumivants; ou bien la formation d'un seul embryon morphologiquenent demi ou entier, si l'on hue deux bastomères contigus; ou bien la formation de deux embryons morpholngiquement remis ou entiers, si l'on tue deux blastomères opposés. En lout cas les emmyons seront morphologiquement denis, si leur plan de symélrie coincide avec un des deux premiers plans de

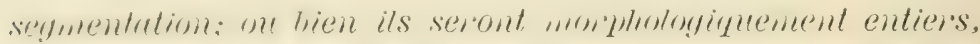
si celle coincidence n'existe pas.

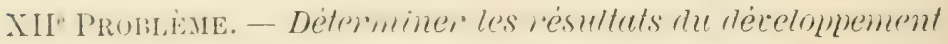
corsquion tue trois des quatre premiers blastomères.

Solution. - Quel que soit le blastomère survivant, il se léroloppera parlaitement comme s'il était isolé. Je renroie done à la solution du problème IIL. Mais, dans ce cas, il faut truir compte de l'emprechement que les hastomères tués et anderemts peurent opposer i la formation des groupes bilatteranx, lorsuge le blastomime de la phatse limite et le groupe cellulaire ménian qui en dérive sont tout-it-fait contigus a un 
des deux plans de sermpntation: cinr, dans ce cas, il ne pourra se former un embrion morpholuginuenent entier, mais seulement un demi-embryon.

Par consé.quent nuns purons établir tout simplement, que si lon tue trois des quatre premiers blastomères, le déreloppement du blastomère survivant donnera lieu i la formation d'un embryon morphologiquement demi, si le plan de symétrie coüncide avec un des deux plans de segmentation, ou morphologiquement entier, si celle coüncidence n'existe pas.

Ise lecteur comprendra partaitenent que la détermination exicte des résultats de ces prollinmes est trés dillicile, parce que l'athésion des blastomères taés joue un role très important dans la formation de l'embryon, rôle que nous ne pourons déterminer que d'une manière générale. Nous savons, par exemple, que la valeur de la surface d'adhésion exerce sur le déreloppement une action qu'on ne peut négliger complètement. Or, cette arlhésion, que nous arons théoriquement supposée constante aussi hien arant qu'après la destruction des blastomères, peut, en pratique, changer plus ou moins et conduire par là à des résultats quelque peu différents.

Mais si l'on tient compte de cen causes ineritables d'erreur's, si lon pense que les résultats que je riens de keterminer sont tout-il-fitit thémriques et, par suite, qu'il doirent itre acceptés comme tels et jugés cum giano salis, le lecteur pourra constater qu'ici encore, il existe mire ces résultats théoriques et les résultats expérimentaux une coincidence parfaite.

Nous devons à Chabry les premières expériences sur cet intéressant sujet (1); mais malheureusement, lat plupart des résultats ne sont pas parfaitement semblables à ceux que je viens de déterminer au point de rue théorique. Cela est dû surtont aux phénomenes qui surriennent dans les neufs dess Ascidies apres lat mort naturelle ou lat destruction piar piquire

(1) Cenabry L. - Contribution ì l'embryologic normale ot lératologique des Ascidies simples, iu: Jouru. do l'Anat. et de la Plrysiol. 1887, p. 291. 
d'un des biastomeres. Ainsi que chabre même le fut observer sourent dans son tratrail, les blastomères de ces animanx, étant pou whèrents, peurent glisser facilement l'un sur l'autre; l'atutre bart, le ou les blastomeres tués ne conservent plus la mene adhésion quils araient aluparavant aree les autres blastomires, le sorte que ceux-ci, se léplacant d’après les conditions mécaniques anxumelles ils obéissent, perdent leur position rédproque, en en prenant une autre qui peut évidemment modifier les résultats du développement.

Quoique Chabrr, en décrivant les embryons obtenus de ses "xpériences, les appelle demi-individus, un quart, deux quarts, trois quarts d'individu, il ent néammoins évident que ces expressions ne se rapportent pas a lat villeur morphologique réelle des enthryons, mais seulement it la valeur de ceux-ci par rapport an numbre des blastomères dont ils dérivent. Car, si l'on axanine les descriptions et les figures de ces embryons, on peut ficilement se convaincre, à quelques exceptions près, qu'il ne s'ignit pas de demi-individus, ou d'un quart, de deux quarts, de trois quarts d'individu, mais presque toujours d'indir idus morphologiquement entiers, quoique plus petits que les normaux.

Je suis donc, à ce point de vue, parfaitement d'accord avec Indescri mais je dois arouer que les résultats des expériences que ce sarint Biologiste a répétées sur ces mèmes animalux (1), en rue de confirmer ou d'infirmer les résultats obtenus par Cimbir, ne peurent aroir ancune valeur dans cette question.

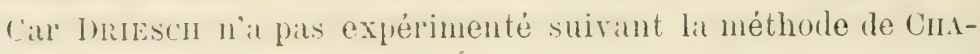
BRr, en tuant les blastomères par piqûre et en les laissant "n place, mais en secouant les oeufs sementés, dont il obtenait des blastomires isolis ou des groupes de blastomeres. Or, nous avons vu que, dans ce cas, les résultats ne sont pas absolument semblables à ceux qu'on peut obtenir dans les conditions spéciales que nous venons de supposer.

(1) Driescu I. - Fon der Entwichelung einzelnor dscidienblastomeren, in: Arch. f. Entwickelungsmech. I Bd., 1895, p. 398. 
Du reste, les expériences de Cul1Bky présentent un intérêt spécial, parce que lat destruelon des blastomeres a éte fate dims toutes les combinatsons pmsibles, precisinent comme nous l'arons fait daus les solutions précédentes, et il est rexpettahle que les résultats natient pas été dicrits plus minutieusement, et que les animax chusis, ne se pritent pis le mieux pour l'étude de cette intéressante question, à causc du glissement et du peu d'alhésion de leurs blastomères.

Ainsi, par exemple, Chabry décrit la formation d'un embryon obtenu apres la mort de deux des quatre premiers blastomeres pris sur une mine diatgnale. An lieu de denx petits embryons entiers, il n'a obtenu qu'un seul embryon; mais il fatut remarquer, comme il le fait ressortir lui meme (1. 2u)t, que les blastomères morts, au lieu de conserver leur position normale, «s'écartent et laissent les deux blastomères virants s'accoler largement », ce qui est suflisant pour nous expliquer la formation d'un embryon seul.

En effet, dans une autre expérience semblable, où « les deux cellules demeurées rirantes se déreloppent chacume pour son compte, sans s'accoler » (p. 205). CiLBBry a vu dériver de chacune de ces deux cellules une blastula et une gastrula et, par suite, deux embryons, lont le déveloprement cepremlant n'allait pas au delá.

Les expériences de Rocx sur les oeufs des Amphibiens (1)

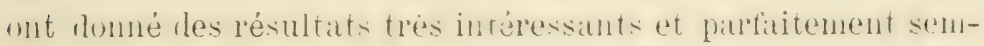
blables à mes résultats théoriques; et cela, grâce à la plus forte adhésion des blastomères et surtout, je crois, à la présence le la memhrane vitelline, qui serr trè bien a matintenir les bastomères dans lemp position primitive, mine apris lexpérience.

(1) Roux IV. - Ueber die kiinstliche Horvorbringung "halber „ Embryonon durch Zerstörung einer der beidon ersten Furchungszcllon, sowio über Nachontwichalung (Postgeneration) der fehlonden Körperhälfte, in: Virchow's Archiv. 11.1 Bd., 1888. 
On peut facilement constater et rẻrifier, par l'examen de ce

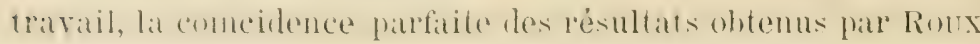
avec mes résultats théoriques.

Roux a obtenu de véritables demi-embryons latéraux (Hemiemiriyones latèrales) en détruisant l'un ou l'autre des deux premier's blastomères (Hemi-emrnoyo dereter et sinister); mais chans ce cas, le plan de symetrie de ces demi-embryons councide tomions arec le mermier plan de segmentalion.

Il a obtenu aussi des embryons qu'il appelle demi-embryons antérieurs (IIemi-embryones anteriores); mais il est facile de se convinincre que ces prétendus deni-enubryons antérieurs no smot, an contrine, que des embryons morphologiquement entiers, ainsi que Hertwig et d'autres Biologistes l'ont déjà fitit remarquer.

Mais ce qu'il importe surtout d'observer dans ces derniers embryons entiers, c'est que leur plan de symétrie est perpendiculaire au premier plan de segmentation, précisément comme j’en ai donné la démonstration et la détermination exacte. Ce fait, qui naturellement ne plaide pas en faveur de la théorio de la Mosaïque de Roux, a été interprété par ce sarant Biologiste comme la conséquence d'un anachronisme dans la formation des plans de segmentation, en supposant que, dans ce cas, le plan de segmentation qui apparemment est le premier, est en réalité le deuxième!

Quoi qu'il on soit, il est rrai pourtant qu'on n’a jamais pu ohtenir jusqüici des demi-embryons postrieurs; call l'embryon que Roux a décrit (p. 136) et figuré (tab. III, fiç. 12) comme demi-embryon postérieur, est plutot un embryon morphologripuenent entier, quoique $\frac{3}{1}$ senlement du nomal par rapport à la masse du corps.

Si les résultats des expériences de Hertwig (1) ont été

(1) Hentwig O. - Ueber den Werth des erston Furohungszellen fïr die Organbilung des Embryo, in: Arch. fomikrosk. Auat. 42 Bd., 1893, p. 739. 
contraires a ceux obtenus par Roux, il faut en chercher la canse dans les conditions spéciales du développement. En examinant les figures annexées au mémoire d'Hertwig, nous pourons constater ficilement que le déreloppenent a domné lien it la formation dembryons morphologiquenent entiors, toutes les fois que le blastomère tué se tournait en bas après l'opération. Mais lor'sque ce phénomène n'avait pas lieu et qu'il ne se formait qu'un véritable demi-embryon, le plan de symétrie de celui-ci coincidait avec le premier plan de segmentation, ainsi que nous pouvons le voir, par exemple, dans quelques-unes des figures susdites.

L'importance du tournement de l'oeuf dans des expériences

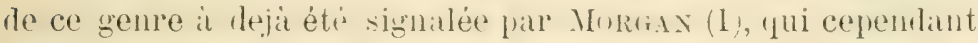
n'en a pas recomu la valeur exacte. Se basant, d'une part, sur des observations de Bors, qui démontrent que si l'oeuf, avant la segmentation, est tourné pôle blanc en haut, il se fait une rotation du vitellus due aux différences dans le poids spécifique des matériaux vitellins, et, d'autre part, sur des observations de $\mathrm{O}$. Schultze démontrant que si l'oeuf, au stade de deux blastomères, a son pòle blanc dirigé en haut, chaque blastomère donne naissance it un embryon complet, Morgay crut que les différences entre les résultats obtenus par Roux et Hertwig dérivaient de la position que l'oeuf prenait après l'opération. Il a donc expérimenté en tuant un des deux premiers blastomères et en laissant développer les oeufs ainsi opérés, partie dans leur position normale, póle noir au dessus, partie retournés pôle blanc au dessus; mais il a obtenu des embryons entiers et des demi-embryons aussi bien dans un cas que dans l'autre.

Mais il laut remaryuer que, dans les expériences d'Hertwit, le blastomère tué tournant en bas, l'oeuf ne faisait pas un demi-tour $\left(180^{\circ}\right)$, comme dans celles de Morgan, où il était

(1) Morgan T. H. - Half-Embryos and Irhole-Embryos from one of the first two Blastomeres of the Frog's Egg, in: Anat. Anz. X Bd, 1895, p. 623: 
retourné poile blane en haut, mais seulement un quart de tour $\left(10^{\prime \prime}\right)$, ce qui est de la plus grande importance. Car, dins ce cas, ainsi que je l'ai démontré, le blastomère de la phase

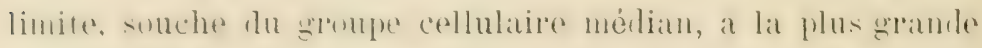

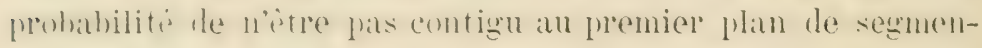
lation et, par suite, le léveloppenent peut tres probahlement domer lien it lat formation d'm embryon morphologiquenent entier; tandis que, dans les expériences de Morgan, la probabilité que peut aroir ce blastomère de la phase limite de se trouver contigu au premier plan de segmentation est à peu près la mème, aussi bien dans les oeufs à pôle noir au dessus que dans les autres à pole blanc en haut.

D'ailleurs, si les demi-embryons obtenus des oeufs a pòle noir en haut ont été plus nombreux que les demi-embryons obtenus des oeufs retournés, cela est dû moins au renversement de l'oeuf qu'aux conditions mécaniques de pression auxquelles celui-ci est soumis dans ce genre d'expériences, ainsi que nous le verrons dans les problèmes suivants sur l'action de la compression penlant le développement.

ENDREs et WALTER ont obtenu, eux aussi, la formation de véritables demi-embryous (1) en tuant un des deux premiers blastomères; mais dans ce cas encore, ainsi qu'on peut le vérifier par les figures annexées à leur travail, le plan de symelirie de l'entryon coüncide arec le premier plan de segmentation.

§ VIII.

Des effets de la compression sur le développement on'ogénétique.

Au chapitre IX de la I Partie de ce trarail, j'ai démontré que les directions des plans rle segmentation ne sont déter-

(1) Exures II, n. Waltuk II. E. - Anstichersucho an Eiem ron Rent fusca, in: Arch. Entwickelungswech. In Bd., 1896, p. 38. 
minées que par les comitions mécaniques mimes dans lesquelles l'neuf" so troure pendiunt son léveloppenent, et rue ces directions penvent itre connues done maniere mathématiquement précise, pourru que l'on tienne un compte exact de la valeur de ces conditions. 'e qui tiait qu'en qartant le ce méme

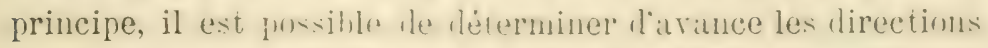
des plans successifs de sementution des differents veufs, uon seulement dans les conlitinn normales, mais aussi daus des conditions artificielles, ceet-it-lire lorsque les oeufs sont soumis it une compression quelennute lans tous les sens possibles.

Je ne reviendrai done pas sur ce sujet et je renverrai le lecteur i ces determinations. Ce qu'il faut maintenant examiner, e'est l'muportance que les directions des plins de sermentation peuvent aroir daus le dérelupuenent ontogénétirque. Cette question, que je posais lejja en terminant la I" partie de ce trarail, peut être a frésent risolue d'une maniere ansi simple et facile que rationnelle et scientifique.

Que la direction de- plans de sermentation ait une ingurtance capitale dans le hévelnpenent ontogénétique pour les interprétations de l'ontrrénese qui se bascht sur l'anisotropisme de l'oeuf ou sur le rrincipe l'une préformation quelconque de l'organisme dans l'veuf, cest ce qu'on comprent très fatcilement. Mais il est évilent qu'plle ne peut aroir qu'une importance tout-it-fat secondaire damo mon interprétation, ou le principe de la localisation dre ditlérenciations est absolument indépendant de lat constitution nurpholurique de l'oenf.

Le lecteur comprendral partaitenent, apres l'exposition des principes de mon interprétation que jai donnés dans les chalpitres précedents, que le dévelnnmment monnlique, étant une

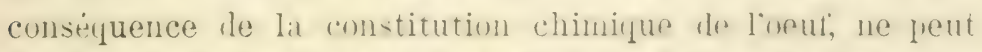
subir ancune modification de la patrt des directions difrérentes

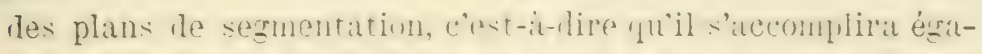
lement, quelles que suient les conlitions mécuninues anissint sur la segmentation de linenf, et que le résultat final seria toujours parfaitement le même. 
I. directions des plans de segmentation dans les oenfs les divers animaux n'ont d'autre affet que de déterminer la place que les hlastomires peurent occuper dans l'agrégrat cellubire de sermentation. Encore faut-il remaryuer que cette place n'est pas toujour's immuable, vu que les blastomères peureut grlisser les uns sur les autres et, par suite, se déplacer de leur position minitive. Par conséquent, le seul effet que la segmentation peut prołuire dans mon interprétation, c'est de déterminer la place que le blastomère de la phase limite vecupera lans l'agrégat eellulaire de segmentation à l'achèvement de celle-ci. Et comme de ce blastomère dérive la ¿ lignée des cellules formant le groupe cellulaire médian qui déterminera le plan de symétrie tu futur embryon, il est évident que le seul eflet de la segmentation sera de áterminer l'orientation de l'embryon par rapport au plan de segmentation.

Mais, quelle que soit cette orientation, elle.n'a qu'une importance tout-i-fiat secondare dans l'ontogénèse; car le développement de l'embryon ne se fait pas moins régulierement.

Par conséquent, nous pourons conclure que lu direction des plans de segmentation de l'oeuf ne peut avoir d'autre résultat qu'une orientation déterminée de l'embryon, par rapport à ces plans.

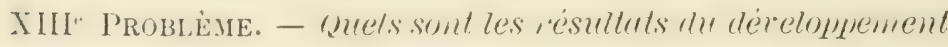
ontogrnelique, si l'oent est soumis a une conturession arant le commencement de sa segmentation?

Som ritux. - Apriss Jes consirlérations que je riens de faire sur l'importance des directions des plans de segmentation dans le développenent ontogénétique, on comprend facilement que, la compression de l'veuf n'arant d'antre effet que la dériation le ces plans de leur direction normale, les iésullats du dére-

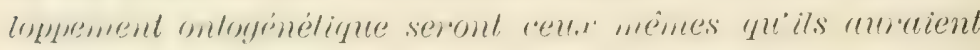
élé dans les conditions nommales, sauf; bien entendu, une

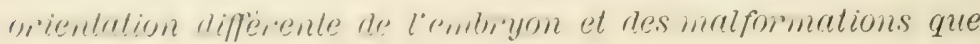


la. compression mêne peut produive en agissant mécaniquement sur le corps de lorganisme.

XIVe Problème. - Déterminer les résultats du dévelop-

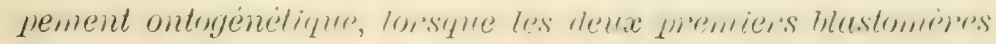
sont soumis a une compression.

Solutron. - Il faut considérer deux cas possibles: $1^{0}$ ) la compression s'exerce dans une direction parallele au premier plan de segmentation; $\stackrel{20}{\sim}$ ) la compression s'exerce dans une direction qui n'est pas parallèle an prenier plan de segmentation.

$\left.1^{\circ}\right)$ La direction do la compression est parallele au premier plan de segmentation.

Ians ce cas, il se produit un phinomène mécanique de la plus grande simplicité, mais en même tempns, de la plus haute importance, dans les résultats du léveloppement.

Nous sarons que, dans toute segmentation, les deux premiers blastomeres adhérent entre eux par une surface plus ou moins grande. Cette adhésion loit aroir we certaine valeur, sans quoi, la segmentation ultérieure des leux blastomères se faisant dans chacun d'eux inrépendamment de l'antre, il ne se forme pas une seule, mais deux carités de segmentation et, plus tard, deux embryons (V. \& T).

Or, lorsqu'on soumet les leux preniers blastomères it une compression s'exerçant dans unt lirection parallèle au premier plan de segmentation, éridemment ces deux blastomères tendront it prendre une forme discoide: et si cette forme était atteinte, les deux blastomeres ne pourratient itre en contact réciproque que par un print seulenent de leur surface. C'est dire qu'ia cause de la pression, la surface d'adhésion des deux blastomères tend it diminuer et it se réduire it un point seulement.

Il faut rematrquer cependint que si l'oeuf est pourru d'une membrane brute, ne prenint aucune part il la segmentation, cette membrane qui enserre les deux blastomeres s'oppose it 
leur déformation et diminue par suite plus ou moins l'effet de la compression.

Quoi qu’il en soit, le deuxieme plan de segmentation pourra encore avoir sa direction mormale, c'est-it-lire perpendiculaire an premier plan et, en même temps, perpenticulate aux surfaces des corps comprimants. Mats il n'en sera plus de mème du troisième plan de segmentation.

En effet, celui-ci derrait itre normalenent perpendiculaire aux leux premiers plans, et, par conséfuent, dans ce cas, parallele aux surfaces des corps comprimants. Mais comme, a cause de la compression, celle-ci étant supposée sullisimment forte, cette direction parallèle n'est plus possible, il sera plus ou moins incliné sur les surfices comprimantes, et, par suite, plus. ou moins incliné sur les deux premiers plans.

Il pourra mème arıiver, si la compression est très forte, que le troisième plan soit perpeudiculare aux surfaces comprimantes et, par suite, perpendiculaire à un seul des deux preniers plans. Inans ce cas, comme la surface d'adhésion des deux hastomeres placés du même còté du premier plan de segmentation est plus grande que la surface d'aulhésion des hlastomères placés de chaque côté de ce premier plan, le troisième plan ne sera pas perpenticulatre au premier, mais au deuxième plan de segmentation.

Il pourra donc arriver qu'au lieu de se former une seule cavité de segmentation, il s'en forme deux, d'un côté et de lautre du premier plan de segmentation. Dans ce cas, les deux premier's blastomines, tout en restant soudés le long du premier plan, se divelopperont néanmoins indépendamment l'un de l'autre, comme s'ils itatient à peu près isolés et donneront lieu chacun it la formation d'un embryon morphologiquement entier, mais plus petit que le normal, et, par suite, l'neuf produira deux embryons soudés entre eux.

Loorientation réciproque de ces deux embryons dépendra de la place que le blastomère de la phase limite occupera dans charque moritié le l'oeul' et, lar conséquent, elle pourra être 
très diffélente, cette place dépendant de lat raleur de la pression et d'autres canses que nous no pouvons comaitre parfaitement. Aussi, la séparation des deux embryons pourra itre plus ou moins complète. Je veux dire, en d'autres termes, que, tout en restant soudés, les deux embryons pouront presenter deux corps entièrement distincts, ou hien deux corps soudés le lony de leurs flancs, ou bien encore un corps senl arec deux tîtes ou arec deux queues.

$2^{\circ}$ ) La direction de la compression n'est pas parallèle au premier plan de segmentation.

La pression pourra donc itre perpendiculate au prenter plan de segmentation. I)ans ce cars, la surface d'alinésion des deux premiers blastomeres ne tendria pas i diminuer. Elle tendra, au contraire, à s'accroitre, les deux blastomères étant pressés l'un contre l'autıe. Il ne se lor'mera done qu'une seule cavité de segmentation et, plus tard, un seul embryon.

En conclusion, nous pourons établir que, siles derr premiers blastomères sont soumis à une compression, les résultats du

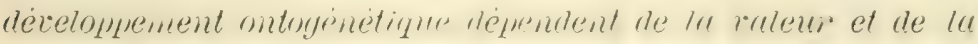
direction de cette pression. Si celle-ci s'exerce dans une direction qui n'est pas parallèle au premier plan de segmentation, le développement de loeut ne donnera lieu, très probablement, qu'à un seul embryon; si, au contiaire, elle est parallèle a ce premier plan, il pourra domner lieu à un embryon seul, ou bien à deux embiyons soudés entre eux, ou

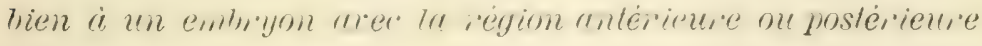
bifurquée. Cela dépendra de la valeur de la mession et de l'ensemble des conditions mécaniques agissant sur l'oent pendant son développement.

Le lecteur comprendra parfaitement que je ne puis insister davantage sur les résultats possibles de ce genre d'expériences. Ceux-ci sont en efret étroitement dependants de lit valeur exacte des conlitions mécaniques dans lesquelless se troure loeuf, et, par consimuent, non seulement le la raleur de la pression, mais ansisi de la présence et de l'extensibilité 
plus ou moins grande de la membrane brute de l'oeuf, et de l'aptitude ou de la facilité qu'ont les blastomères de glisser l'un sur l'autre sous l'action des pressions extérieures. Car il est érident que, si les blastomères peurent glisser facilement, ils peuvent se déplacer de la position qu'ils derraient occuper par suite de la direction des plans de segmentation, ce qui, par conséyuent, doit modifier les résultats du léreloppement ontogénétique.

Les problèmes de ce genre ne peurent donc être résolus d'arance d'une manière générale: car leur solution exige la connaissance exacte d'une foule de données qui ne sont pas les mèmes dans tous les cas. Il faudra donc les résoulre tour it tour, it mesure qu'ils se présenteront et que le Biologiste pourra connaitre exactement toutes les conditions mécaniques et physiques exerçant une action plus ou moins importante sur les résultats de la solution.

Mais si la connaissance exacte de ces conditions est possible, le Biologiste pourra se convaincre que rien n'est plus facile que la détermination des résultats du développement ontogénétique d'aprés mon interprétation de l'ontogénèse.

\section{S IX.}

De l'action de la gravité sur le développement ontogénétique.

J'ai dit au chap. III, que, même en refusant à l'oeuf toute organisation ayant la moindre relation avec la structure de l'embryon qui en dérivera, on ne peut néanmoins aftirmer que sa constitution soit parfaitement homogène: car nous voyons que, dans la plupart des oeufs, les parties qui le forment, bioplasma et deutoplasma, ont généralement une distribution spéciale, que nous pourons aperceroir très facilement par nos noyens d'ohservation. I'ox la distinction des oeufs en alécithes, talolecithes ret centrolécithes, basée précisément sur la diverse distribution de ces deux substances. 
Or, je ne reux pals exclure absolument que des causes physico-chimiques spéciales (adhéxion, cohésion, ou autres) dépendant de la constitution chinique des particules de l'veuf, puissent aroir leur part dans la structure de celui-ci, mais je crois que, dans lat phupart des cass, la grarité est peut-être, sinon la seule, du moins la cause mincipale de la distribution des substances de l'oeuf.

Le bioplasma, crénéralenent plus léger, se place donc en haut, le ritellus nutritif, plus lomd, se place en bas et le vitellus formatif, ayant une densité moindre que le bioplasma et plus grande que le vitellus nutritif, prend une position intermédiaire.

Cela nous explique parfaitement pourquoi, lorsque les oeufs télolécithes sont rentersés sens desis-dessous, la distribution réciproque de ces substances se rétablit normalement, ainsi que Bory (1) a pu le constater directement dans l'oeuf de grenouille.

Mais ce retour automatique de ces substances a leur distribution primitive n'a pas four but, ainsi qu'on pourrait le croir de prime aboril, la reconstitution de la structure de l'oeuf en rue de lat direction des plans de segmentation et du développement ultipieur: il n'est au contraire qu'un phénomène inéritable, dì a l'action le la gravité et à la différence de densité des substances contenues dans l'oeuf et donées d'une parfaite mobilité. ¿e phénoméne a donc bien une cause, mais il n'a pas une finalité.

D'ailleur's, quelle que soit la distribuion des substances de l'oeuf, il est érilent qu'elle ne peut arrir aucune influence dans l'interprétation de l'ontogénèse, telle que je riens de l'énoncer dans les chapitres précélents. Le développement monodique, hase de mon interurétation, est le résultat de la constitution chiminie le l'oeuf, et celle-ci ne peut changer par suite du simple renversement de l'oeuf.

(1) BoRx G. - Biologische Untorsuchungen. Ueber den Einfluss der Sohwere auf das Froschei, in: Arch. f. mikrosk. Anat. Bu. 24, 1885. 
I.orsue, lat sermentation s'accomplissant, se forment les dirers blatstomeres ayant chacun son bioplasma, nous pourons alor's supposer deux choses: ou bien los bioplasmas des blastomères ont la meme densité, ou bien ils ont une densité diffërente. Mais conme de chauue division d'un blastomère en lérivent deux autres diflérents entre eux et différents du précédent, il est trés probable que leur densité n'est pas égale. bar conséquent, la densité du biophasma du blastomere le plus arancé dans l'évolution de l'veuf pourra être plus grande ou plus petite que li densité du bioplasma de l'autre blastomère. Si nous la supposons plus petite, il en dérivera nécessatirement que, lans l'agrégat de segmentation, les hastomères les plus arancés dans l'érolution de l'veuf se troureront accumulés an poile supérieur de l'oeuf, comme je l'ai supposé dans les figure: 1-1!) et que le premier blastomère arrivé al la phase limite se trourera, lui aussi, d ce pôle: si au contraire nous la suppusons plus grande, les blastomères les plus arancés s'accumuleront au prile inférieur de l'oeuf. Mais, quoi qu'il en soit, le déreloppement ontogénétique ne se poursuirra pas moins, tout comme nous l'arons exposé: car, dans mon interprétation, chayue phase de l'ontogénèse est dépendante de la constitution chimique du bioplasma du blastomère qui est son point de départ, mais indépendant de la position que ce blastomère peut avoir dans l'agrégat cellulaire.

I'ar conséquent, si, par des moyens quelconques, nous forcons les hastomères a se déplacer de la position qu'ils occuperaient nomalement at cause de la mrarité, ce déplacement n’aura llatutre considquence qu'une position du blastomère le plus avancé dilférente de lat normale, ce qui n'empêchera pas que le héreloppenent ontrgénétique s'accomplisse normalement.

sionliak, dins des expériences très intéressantes (1) qui ont

(1) Scrubze O. - Dio kïnstliche Erzengung von Doppelbildungen bei F'rosohlarven mit Hilfe abnormer Gravitationswiskng, in: Arch. f. Eutwickelungrmech. I Bd., 1895, p. 269. 
été répétées par WeTzer. (L), a obtenu. par le renrersement des oeufs de grenouille comprimés entre deux lanes dr verre horizontales, des embryons doubles. Mais je crois que ceśs résultats sont dus moins ¿a l'action le lat gravité qu’a lat connpression a laquelle les oeutis ont été soumis. Comme le renrersement arait lieu afrès lat formation du premien sillon de segmentation, il est tris frobatble que le houlerersement des particules deutoplasmatiques contenues dans chacun des deux premiers blastomères a dinninué laalhésion le ceux-ci en les rendant plus indépendants l'un de lautre dans leur direloppement. I'ou la formation de deux enbryons, tout connes'ils étaient parfaitement isolés. On peut ell effet constater, par l'examen des figures annexées an trarail de sincitye, que le premier sillon de sermentation, dans les oenfs où le déreloppement a donné lieu à des embryons doubles, est très accentué et divise en deux moitiés presque indépendantes l'agrégat de segmentation.

Quoi qu'il en soit, ces expériences ne pourront aroir une importance decisive dans cette question qualor's seulement qu'elles seront faites sur les oeufs renversés sans itre sounis . à une pression quelconque.

Nous pourons donc conclure que la giveité n'exerce sur

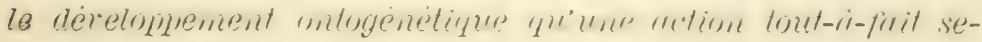
condaire, en tant quelle peut produire un arangement dé-

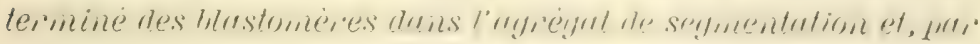
suite, une orientation déterminée de l'embiryon par rapport au piemier plan de segmentation; mais que celle action n'a aucune influence sur lo développentent noinal de l'embryon.

Que la gravité ne soit pas nexessaire an déreloppement ontogénétique, c'est ce que Rocx a d'ailleurs démontré par des expériences contenahles, et cent ce dont nous pourons,

(1) Wetzec G. - Veber die Bedeutung der cirkuliren Furche in der Entwickelung der Schultze'schen Doppelbildungen ton liana fusca, in: Arch. f. mikrosk. Anat. 46 Bd. 1895 , p. 654. 
d'autre part, nous convaincre ficilement par la simple considération que les neufs de certains animaux ne se développent pas moins normalement, yuoi qu’ils se trourent dans des positions tout-it-fait diflérentes par rapport a la direction de la gravité.

\section{$\S \mathrm{x}$.}

\section{De l'action des agents physiques et chimiques sur le développement ontogénétique.}

Yous arons ru, dans les chapitres précédents, que le développement ontogénétique est, d'après l'interprétation que j'en ai donnée, un phénomène essentiellement chimique.

La potentialité érolutire de l'oeuf, et, par suite, la formation et la nature des deux premiers blastomeres et de toute la série des autres cellules caractérisant la première lignée cellulaire; la constitution de substances sécrétées par les blastomères pendant leur formation, le rythme même de segmentation sont autant de phénomènes qui ont leur base dans la constitution chimique du bioplasma et du deutoplasma de l'oeuf.

La potentialité qu'ont les blastomères de suirre une autre érolution domnant lieu à la formation d'autres cellules et, par suite, d'autres lignées cellulaires, tient, ainsi que nous l'avons ru, a la constitution chimique du bioplasma de ces blastomères d'une part, et des substances produites par la probiose des blastomères d'autre part. La différenciation histologique est étroitement dépendante de la nature bioplasmatique des cellules, ainsi que je l'ai rénontré; la différenciation même morphologique, quoique répendant d'une foule de conditions méraniques qui peurent nous échapper dans plusieurs cas, est néanmoins liée, elle ausi, a la constitution chimique des cellule: formant les organes et déterminant par leur forme et far leur: flnoprietés physico-chiniques, sinon tous, au moins la plupart des caractères distinctifs de lorgane. La locali- 
sation des proliférations cellulatires et les diffẻrenciations morphologiques et histologriques dépent de lia présence et de la localisation dans lembryon de cellules qui, par la niture chimique spéciale le lours bivplasnits et des substances constituant le milieu interne orinique, peuvent itre le point de départ pour une nouvelle prolifération cellulaire.

Tous les phénomenes fondanentaux de l'ontogrénèse se réduisent en somme à des phénomènes essentiellement chimiques. Rien done de jlus naturel que les agents capahles de modifier les réactions chimiques des corps puissent produire aussi des modifications plus ou moins fortes sur le déreloppement ontogénétique.

Mais comme ces modifications sont étroitement dépendantes de la nature chimique des sulystances réagissantes entre elles, il est érident qu'elles ne peurent itre prérues ni déterminées d'arance, sans la connaissance parfaite et exacte de la composition chimique de ces substances. Or', malheureusement, cette connaissance nous manulue, et, far consirguent, nous ne pourons préroir ni déterminer exactement les effets de l'action de ces agents sur le développenent ontugénétique; mais nous devons nous en tenir seulement i cette conclusion que les rgents phy-

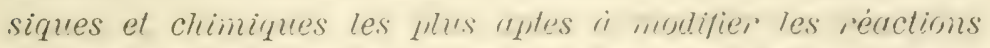

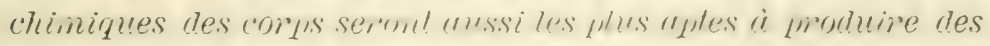

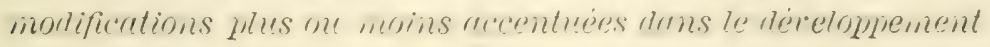
ontogénélique.

Parmi les agents physiques, il taut mettre en prenière ligne la chaleur.

Tous sarons que, dans tontes le reactions chimiques, il y a un mriminmet un minimmm de tenpérature, au delá desquels elles ne peurent flus s'acconmlir, et un ruthurm dans lequel elles s'accumplissent le mieux. Mais les legrés de tempriature caractérisant ces terme- smut étroitenent dépendants de la composition chininue des substances réagissantes at varient far eonséquent pulm celles-ci varient. Le bioplismat et le deutoplasmat de linenf ne se somstrairont lone pas a cette 
loi générale, et nous constaterons, jar suite, que, pour ces deux substances aussi, il existe des dexprés de température inari-

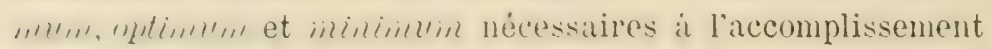
de leurs réations chiniques carrictéristiques de l'issimilation. Et comme la nature chimique du bioplatsma et du deutoplasma n'est pas la mème dans les oenf's des diflérents animaux, il psit tout naturel que les degrés de température ne soient pas les mêmes dans tous les oeufs.

C'est donc en vain qu'on tente de déterminer les points critigues ou les points cardinaux physiologiques de la tempirature it laquelle le déreloppenent on torénétique est possible, ou lu moins, c'est en rain qu'on tente de les cénéraliser: car ces points sont fixés pour chaque espèce d'oeuf, en raison de sir constitution chimique. On comprend donc facilement que si l'on porte un veuf à un degré de température au delà de ses points cardinaux physiologiques, les réactions chimiques de l'assimilation ne pourant plus s'accomplir, la segmentation et, par suite, le développement s'arrêteront.

Il faut néanmoins faire une distinction entre les actions de lit basse et de la haute température, distinction que nous derons faire d'alprés les connaissances que nous possédons sur les phénomènes chimiques des substances brutes.

Une température plus basse que le minimum ou plus haute que le maximum n'est plus sutfisante i l'accomplissement des phénomines chimiques. Mais la basse température, du moins dans certaines limites, n'arrit pas comme décomposante des substances réagissantes; elle ne fait que suspendre l'accomplissement des reactions, sans apporter dans ces substances des modifications capables d'altérer leur composition chimique. Par conséquent, aussitrit que la température s'élèvera au-dessus du mininum, les phénonènes chimiques pourront recommencer et se poursuirre normalement.

l'al' contre, une température au dessus du degré maximum pent hien ne fats arir de la mine manière. Dans les composés peu stables et surtout ditns les composés organiques, elle peut 
modifier notahlement leur comprition chiminue: elle peut

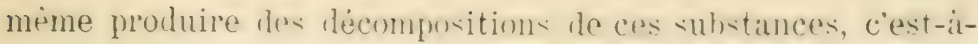
dire les monlifier tellement que lenr con-titution ne suit plus parfaitement la minne quene etait aluparatrant. Halns ce cas, on comprend aisénent que, les sulsstances réarisiantes étant changées, los resultits le lour riaction ne semont plus éraux

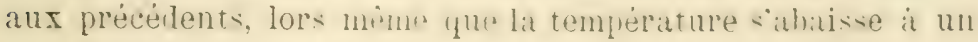
degré compris entre le maximum et le minimum.

En peu de mots, l'abiaissement de lat température atu-dessous du mininum ne fait une -u-pentre l'atcomplissement des réactions chindques, tamlis que l'élération atl-dessus du malximum, non seulenent fent le sispendre. mais, far les altérations qu'elle apporte dans le sulstunces réarisiantes, elle peut mème les empêcher ultérieurement.

En appliquant ces connatsances atux réactions chimiques de l'assimilation s'atcompliscint danc loenf entre le bioplatsmat

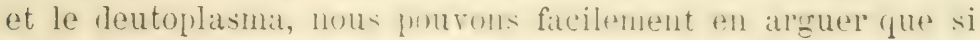
un oeuf est exposé à une température au-dessous du point critique minimun de son divelofpenent, celui-ci sera suspendu. mais il pourra recomnencer ansitrit que les conditions de température seront rederenues farorahles; tamlis que si l'veut est exposé a une temperature superieme it sm puint critique maximum, non senlenent le levehnenent se? surpendu, mais il pourrat meme arrirer gue les substances he loeut, sulbissant des altérations plus ou molus frofonles. perdent leurs propriétes chimiques cartatériviques ot, que par suite, le développement ontogénétique ne soit plus possible.

Nous devons en somme conclure que, le déreloppement de l'oeuf, étant la conséquence des réactions chimiques entie les substances qui le constituent, il est sounis a toutes les actions que la chaleur peut exercer sur l'accomplissement des phénomènes chimiques, et que ces actions sont sufisantes pour

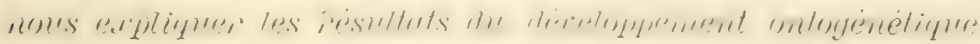

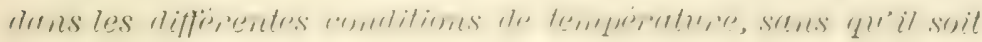
besoin de recourir it des hypotheses spéciales. 
Te lois néanmoins attirel l'attention des Biologistes sur des phénomenes d'une certaine imprtance, yui peuvent se manifester dans ces expériences comme conséquences tout-i-fait naturelles de notre interprétation mème de l'ontogénèse.

I’uisque, dans le déreloppenent monodique, la nature chimique du bioplasma des blistomeres subit une transformation graduelle ì mesure que lo développement progresse, c’est-àdire a mesure que les blastomeres abandonnent une des phases de l'érolution de l'oeuf pour passer à la phase suirante, on comprend ficcilement que les points critiques des diflérents hlastomeres peurent bien n'ètre pas les mèmes pour tous. La possibilité de cette difrérence plus ou moins grande entre ces points critiques dépend de la diflérence plus ou moins grande entre les constitutions bioplasmatiques des blastomères. Il est donc très probable que cette difrérence existe réellement, du moins entre les premieres et les dernières phases de l'évolution de l'oeuf; et cette probabilité s'accroit encore si nous consilérons les blastomeres dans les premières phases de l'érolution le l'veuf et les cellules de la 2 , de la 3" etc. lignnée cellulaire: car dans ce cas, les différences dans la constitution bioplasmatique de ces cellules sont, sans aucun doute, encore plus grandes.

Par conséquent, lorsquon considere un agrégrat de segmentation ou les constitutions bioplasmatiques des différentes cellules ne sont pas parficitement inlentiques, les points critiques do l'agrézat tout entier seront compris entre les points critiques de ces cellules.

Concrétons mieux ces considérations par un exemple.

Supposons que les points critiques de l'oeuf a soient $0^{\circ}$ et $30^{\circ}$ ot que ceux du bastomère a la phase limite 1 soient $10^{\circ}$ et :3.", et supposons encore, pour plus de simplicité, que les points critiques des phases intermédiaires soient compris entre $0^{\circ}$ et 35." Les points critiques le l'argregat tout entier seront naturellement $10^{\circ}$ et $30^{\circ}$.

En effet, tant que lat température se maintiendra entre ces 
degrés, les réation- he l'asimilation de chatue blastomère seront possibles et, fatr suite, le déreloppenent se poursuirra rérulièrement. Matis, si l'on forte l'arrugat it un degré audessous de 10", frar exemple it s', les réilctions le l'assimilation pourront se fatre nomalement dans les blastomeres dont le point critique est am dissous de co deré, mais elles s'arreteront nécessairement dans le blastomère $\%$. Xous constaterons, par suite, un arret dans le réveloppenent ontorénétique, tandis que nous n'aurions pu constater aucun arrêt si, au lieu de l'agrégat de segnentation, nous arions sounis a cette même température de s" l'veuf arant sal sermentation, ou bien au commencement de celle-ci.

Or, comme les modifications morphologiques qui ont lien pendant le dévelopnement ontruénétique sont froduites, ainsi que nous l'arons ru, par les proliférations de certaines cellules, il est clair que si ces proliférations ne peuvent aroir lieu, les formations spéciales yui en lériveraient ne se produiront pas. C'est dire que nous constaterons me malformation, ou une anomalie qui aura pour cauce la rariation de température à laquelle l'embryon à été soumis.

L'action que la lumière peut exereer sur le développement ontogénétique s'explique, elle aus-i, assez facilement par des consirlérations analogues. Nous sarons qu'il y a des substances chimiques dont la réatetion ext farorisée par la lumiere, c'estatrlire que l'action de celle-ci est une comlition, sinon absolument nécescaire, du moins firorahle a son accomplissement. Mais nous sarons encore que ledlot le cette action dépend de la nature chimique des substances réagissantes.

En tout cas, il est une notion acquise par les expériences photu-chiniques, que, parmi les dillérents rayons du spectre lumineux, les rayons hleus, riolets at les ultra-riolets exercent une action poritire sur l'alcomplisisment less réactions chimiques en les lavorisint, tamdis que les autres, et spécialement les rityons jammes, les rouges et les infratrouges, sont chimiquement inactifs. Tous pourons dunc, en partant de ces 
motions et en les appliquant aux substances vivantes, on prevoir les conséduences natubelles datns les expériences biologiques.

Lobscurite pourra donc rester sans action sur le déreloppement de certains ocufs, tindis qu'elle pourra influer sur le développenent des autres. cela dépendra naturellement de lá constitution chimique de ces oenfs, constitution que nous ne comnaissons pas. Mais si cette action existe, il est facile de se convaincre qu'elle se résondra en un ralentissement on on un arrêt du développement, ru que l'olscurité, c'est-it-dire l'absence de lumière, ralentira ou bien empèchera les réactions chimiynes de l’assimilation, nécessaires pour la segmentation de l'oeuf, et, lar conséquent, pour le développement ontogénétique.

Vous pouvons conclure de même pour les rayons inactifs.

Etant armis que certaines especes d'veufs exigent l'action le la lumiere pour l'accomplissement des réactions assinilat trices, il est evilent que les rayons inactifs (jaunes, rouges, infratrouges) exerceront une action analogue a celle de lobscurité, tandis que les rayous actifs (bleus, violets et ultraviolets) agiront analoguement à la lumière blanche.

C'est d'ailleurs ce que Dutrochet, Yung et d'autres biologristes ont pu constater dans plusieurs expériences.

Mais yuoi qu'il en soit, je ne crois pas que la lumiere puisse aroir une action quelconque sur la localisation des fonctions et des orranes dans le léveloppennent ontogénétique. Bien que, dans quelyues cas, il nous semble que certaines formations ot, tout sfécialement, certaines diflérenciations histolorgiques soient provoguées par la lumiere, nons derons néanmoins exatminer attentivement chapue question, afin de ne pas tomber dans des déductions inexactes.

Je ne veux pas nier d'une manière absolue que la lumière juisse axercer une action directe sur certaines différenciations: mais je crois que cette action est toujours subordonnée i la constitution chimigue des substances sur lesquelles la 
lumière agrit. J'armets, par exemple, que la production du pigment dans les cellules est due i l'action de la lumière; mais je crois que cette production est arant tout dépendante de la nature chimique de ces cellules, et de l'action de la lumière en denxième lieu. Il faut toujours se rappeler en somme que l'action physique n'est pas le facteur eflicient du phénomìne, natis sentenent la conlition farorable ou nécessaire pour son accomplissement, et que le facteur rraiment eflicient rivile dims lit constitution chimique des substances.

Si alor's nous consilérons les dillërenciations ontogénétiques à ce point de rue, que je crois scirntifiquement exact, nous royons que, même on attribuant à la lumière une action active dans la détermination d'un caractère histologique, celle-ci est néanmoins subordonnée arant tout à la nature chimique des cellules et at lat localisation le celles-ci dans l'agregat cellulare, lans l'emhryon. Et cette localisation, et cette nature chimique, sonf dépemlantes, nous le sarons, du mode même de dérelnppement qui est lat base de notre incerprétation.

Si donc, dans un entroit queleonique du corps de l'embryon, nous royons, par exengle. paraitre une fache pigmentaire, nous pourons bien admettre que cette tache est produite par l'action de la luniera (ce que l'expérisner jourra lémontrer); mais, en tout cas, la formation et la lecalisation de cette tache sont dues arant tout a lil présence, dals. l'molroit susdit, de

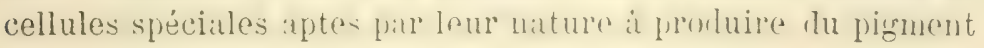
sous l'action de la lumière.

Les expériences de Driescu et de Roux ont d'ailleurs démontré que la lumiere n'exerce aucune action ni sur la direction des plans de semurntation, ni sur lat localisation des différenciations ontogénétiques.

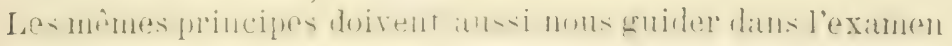
des actions de l'électricité et du magnétisme. Il s'agit ici de

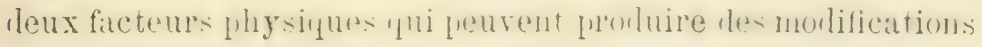


ou des altirations chimigues remalrumbles dams cortaines substances. Il est donc tres probable que si l'on fait agir

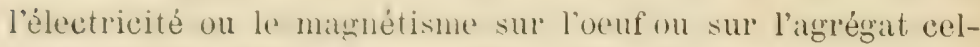
lulaire dérivant de sat sermentation, l'oenf même ou certaines cellules de l'agrégat en subilont des modifications si importantes dans la constitution deri hiomolécules de leur hoplasma, yut les réactions ultériesures, car'actéristiques de leur déreloppenent, ne seront plus pusibles, et que, par conséquent, le développenent ontowénétique en subira rles perturbations pourant amener ou bien l'arret de la segmentation, on bien encore l'arret de la dirision de certaines cellules et, par suite, des malformations ou des monstruosités.

Evidemment, tous ces résultats dépendront directement de la constitution chiminue du bioplatsmat des cellules et pourront, par suite, varier d'alpès les diflérentes espèces d'veufs sur lesquelles on opère.

Pirmi les agents physiques, les phénomènes osmotiques jonent certainement un rile d'une très grande importance. Bien que la nature et l'accomplissement de ces phénomènes tiennent arint tout a la constitution chimique les substances qui les proroquent (ce qui d'ailleurs est aussi vrai pour ceux-ci que pour les autres phénomènes physiques), il est néannoins indiscutable qu’il litut les ranuer dans la catégorie Hes manifestations d'ordre physigue: car les substances qui sont le siege de ces phènonines ne changent pas leur constitution chimique pendant que l'osmose s'accomplit.

Lorsqu'on met un oeuf ou un agrégat cellulaire de segmentation dans des solutions spéciales, les résultats possibles dépendent naturellement de la constitution de l'oeuf, les cellules, de leurs membranes et du liquile anbiant; et comme, dans notre interprétation, les cellules de l'argégat ne sont jas toutes partaitonent irlentigues, il est érident qu'elles fourront hion ne pas se comporter toutes inentiquement par rapport à ce liquide. Quoi qu'il en soit, les résultats seront positifs ou négatifs. 
Or, dans les cas de résultats positifs, nous portons supposer que le liquide externe dilluse à l'intérieur do l'oenfou des cellulos, ou bien qu’une partis des liquilles internes de cenx-ci difluse à l'extérieur. Inans chacun de ces résultats, on peut préroir aisément que lo déreloppement ontogénétique pourra on subir des perturhations plus ou moins fortes. Mais les causes de celles-ci hoirent être rangées en deux catégories: chimiques et physico-chimiques, quoique cotte distinction théorique ne soit pas toujours possible dans la réalité.

II s'arit de causes perturbatrices chimiques, lorsque les substances pénétrées à l'intérieur des cellules peurent produiro dans la constitution hioplasmitipue de celles-ci des modifications plus ou moins fortes amenant son altération chimique et, par suite, l'emprèchement des réactions caractéristiques de l'assimilation, lont la posibibilite et l'orire sont déterminés, nous le sarons, par la constitution chinique du bioplasma. Il y aum, lans co cas, une réritable altération du bioplasma et, par suite, imposibilité du déreloppement ontogénétique, si l'action osmotique à été axercée sur l'oeut, point de départ de tout le déreloppement; ou hien impossibilité partielle, c'est-í-dire déformations plus ou moins fortes, si cette action n'arit que sur quelques-unes des cellules de l'agrégat de segmentation.

Mais, dans ce cas, on comprend que la cause directe de ces résultats résile dans les actions chimiques les substances pénétrées, et que le phénomsne phrsique le l'unmuse n’en est que la cause indirecte, yui a permis lit pénétration de ces substances.

Il s'agit, au contraire, de causes perturbatrices physicochimiques, lorsque les substancess pénétrées il l'intérieur des cellules n’altirent pas la constinution chimique du bioplismat. Cependant, elles peurent hisn en empecher ou en molifier les réactions de l'assimilation.

Nous savons que la cause de toute réaction chimique entre reux molécules résile dans l'alinité le leur's atomes, en 
relation, hien entendu, arec des conditions physiques déterminées. si certains atomes ahandonnent une molécule pour s'unir it une atutre, ou bien pour former des mroupes atomiques diflérents, ce qui constitue précisément une réaction chimique, c'ent que l'atlinité entre le's atomes d'une mème molécule est moindre que laaflinité entre les atomes de deux molécules diflërentes, sans quoi, le détachement des atomes et, par suite, la réaction chimique n’aurait pas lieu.

Or, nous pourons comprendre parfitement que l'introduction, par eflet de l'osmose, d'une substance spéciale dins la cellule, substance dont les molécules s’entremêlent aux biomolécules, puisse emprecher ou troubler les riactions chiniques de l'assimilation, sans promlure une réritable alteration chimique des biomolécules. Il śagrirait, dans ce cas, tout simplement, d'une action physico-chimique, en tant que les atomes de la substance introuluite pourraient modifier par leurs allinités les aflinités des atomes des bionolécules et, par suite, en empècher les réactions, basées, comme nous renons de le dire, sur ces affinités.

En tout cas, quelle que soit la nature les actions dérirant des phénomènes osmotiques, il est néanmoins indiscutable qu'elle dépend absolument de la constitution chimique du bioplasma de l'venf ou des cellules. Par conséquent, comme nous ne comnaissons pas cette constitution, nous ne pourons absolument en préroir les effets, ceur-ci étant étroitement liés a elle, et nous derons nous en tenir uniquement a leur constatation, à mesure que les expériences sur ces intéressants phénomènes progresseront et deriendront plus nombreuses.

Quant anx actions chimiques des substances toxiques on d'autres, nous devons malheureusement arriver it la mêmo conclusion. 'lonte prérision serait nécessairement prématurée, ru que les effets de ces actions sont naturellement dépendantes de la constitution bioplasmatique, que nous ne comnaissons pas encore. Cepentant, si l'on considère que lat base du dérelop- 
pement ontogénétique est essentiellement un phénomène chimique, nous derons en arguer que les actims chiniques doivent ètre classées, sans aucun doute, parni les actions les plus importantes et les plus énergiques jourant influep sul les phénomènes de l'ontugénèse. C'est ce qu'ont démontrí d'ailleur's de nombreuses expériences. 


\section{Chapitre XIV.}

\section{Le développement mixte.}

SOMMAIRE: Les autres développements hétérogénétiques possibles - \$10: Les développements dimonodiques et leurs relations possibles avec le développement ontogénétique des Gastéropodes, des Echinodermes, et des Ctẻnophores - § 20: Les dévoloppements polymonodiques - §30: Les développements cycliques et leurs rapports possibles avec la segmentation.

Nous avons vu au chapitre $\mathrm{V}$ que les modes de développement ontogénétique possibles sont deux: le polyodique et le monorlique. J'espère aroir fait ressortir suflisamment, dans les chapitres précédents, tous les arantages que le développement monodique présente dans l'explication des phénomènes ontogénétiques. Mais comme, dans l'immense variété des manifestations biologiques naturelles, il fiat absolument tenir compte de toutes les conditions possibles, nous devons revenir maintenant í l'eximen de certains autres modes de développement hétérogénétique résultant de combinaisons entre les deux modes fomlamentaux mentionnés. Nous verrons alor's lespuelles de ces diffërentes combinaisons possibles peuvent itre appliquees convenablement a l'explication de certains phénomènes de l'ontogénèse.

§ I.

Les développements dimonodiques.

I. - Le dévelomement dimonodique de $1^{\text {er }}$ ordre. - Supposons done arant tout qu'i la première division, l'oeuf $a$, au lien de domerer origino i denx hastomères dont l'un représente 
une phase de son érolution, et lantre la phave suirante, selon le mole de déreloppenent nomolinue, prorluise, an contraire, deux blastonères dont l'un, h, représente une phise de la méme érolution et lont l'autre ne suit fras ', mais slillérent de celui-ci, ainsi que nous l'arons vu dans lo dérelorpenent folyodique. Nous indiquerons celui-ci par la lettre $\because$. Evidemunent, lal potentialité érolutive de ces leux hlastomeres b, sera diflérente.

supposmis maintenant quaprie cette premiere division, le déreloppement se foursuire selon te mole monolique. Nous pourrons indiquer l'érolution de l, fiar lat série des lettres", $a, e, f \ldots p$, et l'évolution de $c^{\prime}$ par une série de lettres $d^{\prime}, e^{\prime}$, $f^{\prime} \ldots p^{\prime}$, de manière que l'évolution de l'oeuf pourra être indiquée par le schéma suivant:

$1^{\circ}$

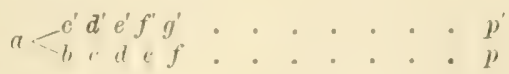

J'appellerai ce mode de développement, où l'un des deux blastomères ne représente pits une des phases successives de l'érolution, le dérelopnenent dinionorlique, et je distinguerati ce premier cas que jer resus de consirlérer, et ou ce phénomène se produit a la preniere division de l'neuf, en l'appelant développement dimonodique de premier ordre.

Exidemment, lans ce cats, l'alégat cellulitire de segmentattion résultera constitué de drux lignéécellubires différentes, aboutissant chacune i une phase limite diverse $p$ et $p^{\prime}$, pourant devenir le point de départ d'évolutions ultérieures et, parl suite, de diflérenciatiom histoluginues of morphologiques différentes.

On comprend aisément dès lor's que l'isolement des deux premiers blastoneres duit donner des résultats tout atutres que dans le dérehntement monorlique: car a l'on isole les deux blastomères $b, c^{\prime}$, chacun d'eux ne pourra aboutir, par

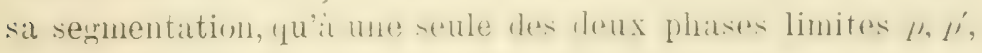
et, par suite, l'embryon qui en résultera (en supposant que le

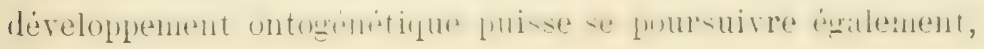


du moins, jusqu’a un certain point) manyuera des difrërenciations monphologrigues at histologrigues dérivant du blastomère

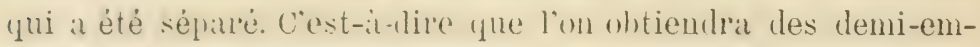

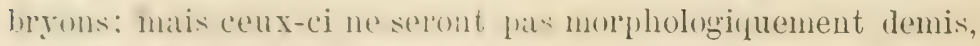
cest-it-dire frésentant senlement la moitié droite ou gatuche du corps, mats quantitationment demis, en tant que lemr corps manyueral de certains organes. Il serait plus exilet de dire qu'ou obtiendra des embryons incomplets.

Je ne sais si, parmi les organismes, il en existe quelques-uns amxquels on puisse appliquer ce mole de déreloppenent. Peut itre en arons nous des exemples ditns les Gastropodes, si nous voulons nous en tenir seulement aux conclusions de CRAMr'Tox (1). Mais je crois, aree Chili) (2), que ces conclusions ne sont pas rigoureusement exactes. Cranrтол dit aroir obtenu, il est vrati, chez Illymmassa, des framments de larves, en isolant les blastomères aux stales de 2, de tou le s; mais cela n'est pas tress certain. Il semble bien, d'apres les figures mèmes de ChAmpon, que, dins quelques cats ses lalres n'étaient pas des demis ou des quarts de larres, mais qu'elles itaient devenues plus ou moins completes. (quoi qu'il en soit, d'autres observations plus rigoureuses sont absolument nécessaires pour résoudre tout-i-fait la yuestion; mais il faudra se rappeler toujour's, daus les conclusions qu'on pour'a dérluire, que le mode de segmentation des blastomires isolés et le nombre le ces blastomères ne sont pas der caractéres sullisants pour jugrer si l'embryon est la moitié, ou un quart ou un huitième de l'embryon total; car nous avons vu que des embryons morphologriquement entiers, dérivés des blastomieres isolés, peurent présenter un mombre de celluler moindre que les embryons mormaux. Tout jugement sur la villemr morphologique d'un embryon doit être

(1) Cianptox H. E. Jr. - Experimental Stuly on Gasteropod Derelopment, in: Arch. f. Entwickehngrmech. III 13d., 1896, pp. 1-26.

(2) Cund G. M. - A preliminery Accont of the cluavage of Arenicola cristats, with remerks on the mosaic theory, in: Zool. Bull. Vol. I, 1897, pp. 71.85 . 
basé sur le nombre et la disposition de ses parties morphologiques.

II. - Le déreloppement dimonodique de 2 ordie. - Supposons maintenant que l'oeuf $a$ se divise normalement en deux blastomères /", ", mais que le bistunere l, ent se livisant a son tour, donne origine i deux blastomères, dont l'un $c$ et l'autre $d^{\prime}$, c'est-à-dire différent de $d$. Faisons en somme pour $b$ la même supposition que nous venons de faire pour a dans le déreloppenent dimonulique de I rrdre, et supposons qu'aprés cette dirision suirant le déreloppenent polyolique, l'érolution utérieure continue suirant le mode monodique. Tous pourons représenter l'érolution de l'veuf par' le schéma suivant:

$2^{\prime \prime}$

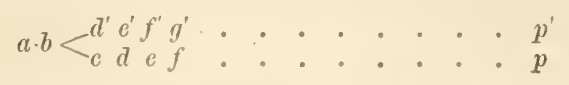

J'appellerai celui-ci le déreloppenent dimonodique de I' ordre. Dans ce cas donc, $a$ se divisera en $b, c ; b$ se divisera en $c, d^{\prime}$; c en $l, e$, comm'il suit:

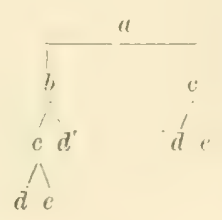

Ainsi qu'on le roit, dans ce cas encore, l'oeuf possède une potentialité érolutire dunble, qui l'amene, par' sa segmentation,

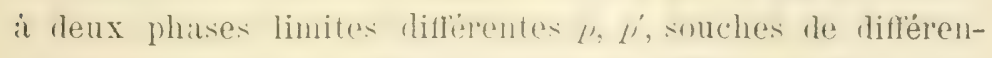
ciations histologiques of morphologiques dillépentes. Mats, dans ce cas, le léreloppenent des hlatsonnines isolés. n'aloutira pas aux mêmes résultats que dans le cas précédent I.

En effet, le blastomère $c$, à lui seul, ne possède que la potentialité érolutive $c . . . j$, tandis que le blastomère $b$ possède les deux sortes de potentialités érolutives aboutissant à $l$. $\not$ ',

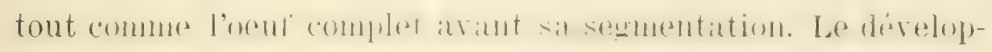
pement de e ne pourra donc donner lieu qu'a un embryon 
incomplet: lo déreloppement de b domnera lieu, au contraire, it un embryon complet, quoique plus petit que le normal.

Les expériences futures nuts diront $s$ 'il $y$ a des organismes qui se déreloppent suivant ce type de développement.

III. - Le développement dimonodique de $3^{\circ}$ ordre. - Faisons maintenant pour c la même supposition que nous venons de faire pour \%. Lor's de sa division, c donnera lieu, non pas à $d, e$, mais a $d$ et à un autre blastomère différent de $e$, que nous indiquerons par $e^{\prime}$. La potentialité érolutive de l'oeuf pourra donc être représentée par le schéma suivant:

3

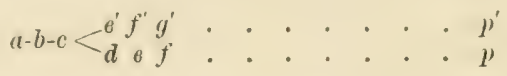

J'appellerai celui-ci le déreloppement dimonodique de III" ordre, et la segmentation de l'oeuf se fera d'après le schéma suivant:

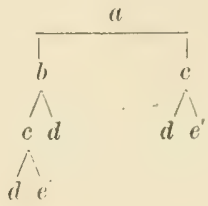

où lim roit que la potentialité érolutive de chacun des deux prenier's blastomères 7, , est égalle ì celle de l'oeuf, tous les deux pouvant aboutir, parr leur développement, aux deux phases limites $/ \prime, f^{\prime}$. Mais il n'en est pas ainsi pour les quatre blastomères c, $l, n, e^{\prime}$ : car le ceux-ci, le blastomère c possède seul la même potentialité érolutive que l'oeuf', pouvant donner lieu par sal dirision aux blastomères $/, e^{\prime}$, souches des deux sortes de potentialités de l'oeuf. Des autres, les deux blastomères d ne peurent aboutir qu'it la phase limite $\mu$, et le blastomère $e^{\prime}$ qu'il la phase $x^{\prime}$.

Le seul blastomère e pourra donc donner lieu au dévelopfrment d'un embryon complet; les autres produiront des enbryons incomplets. 
IV. - Le développement dimonodique de $4^{\circ}$ ordre. - Si nous faisons pour $d$ une supposition analogue aux précélentes, nous pourrons representer lia potentialité érolutive de l'oeuf par le schéma suivant:

$4^{0}$

$$
a-b \div c-d<<_{e}^{f^{\prime}} g^{\prime} h^{\prime}: \therefore: \quad: \quad: \quad: p^{\prime}
$$

et la segmentation de l'oeuf par ce schéma:

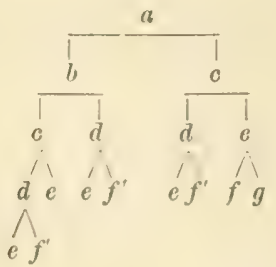

Dans ce cas, on constate que $\pi$ et $c$ ont la même potentialité que l'oeuf; et des quatre blastomères ', $a, r, e$, ce dernier seulement n'a qu'une potentialité partielle; les autres possèdent la mème potentialité que l'oeuf. I’ isolement de celui-ci ne pourra done donner lieu qu'à un enhryon incomplet; celui des autres, a des embryons complets, quoique plus petits que le normal.

$\mathrm{V}$. - Le développement dimonodique de $5^{\mathrm{e}}$ ordre. - En faisant une supposition analogue pour le blastomère e, la potentialité évolutive le l'veut' sora représentée par ce schéma:

5"

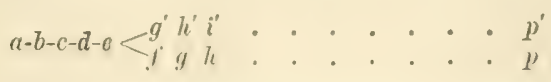

et le résultat de la segmentation par ce schéma:

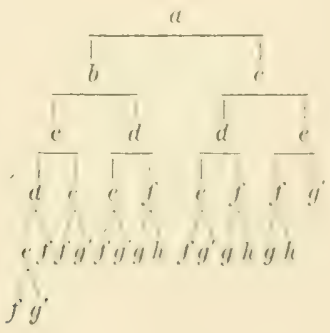


Dans ce cas, les deux blastomères $b, c$ ont la même potentialité érolutive que l'oenf et domeront lieu, par leur développement isolé, à des emhryons complets. Il en sera de même des quatre premier's hastomires isolés. Au contraire, des s premiers hatitmimes, quatre seulement, e'est-it-dire le d et les trois e' ont la mème potentialité que l'oeuf', tandis que les trois $f$ et le $g^{\prime}$ n'ont qu'une potentialité partielle.

VI. - Le développement dimonodique de $6^{\ominus}$ ordre. - Enfin, si l'on fatit une supposition analogue pour le blastomère f, la potentialité érolutive de l'oenf sera représentée par ce schéma:

6

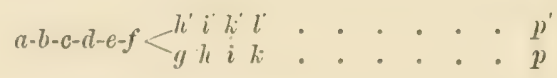

et les résultats de la segmentation par ce schéma:

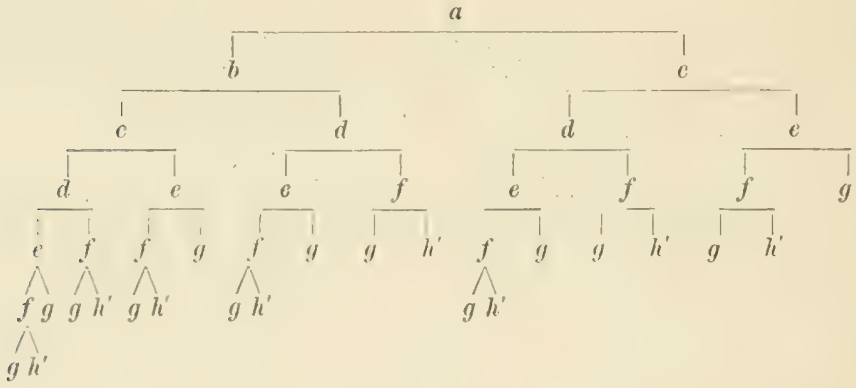

oi l'on roit que les deux premier's blastomères, aussi bien que les quatre prenier's, ont lia même potentialité évolutive que l'oeui, et que des $S$ premier's blastomires, un seul, g, n’a qu'une potentialité partielle.

Il est érident que je pourrais continuer de la même maniere l'exanen des autres diveloppements dimonodiques possibles; mir je crois pouvoir m'arreter ici, ru que les exemples cités sont suflisants pour faire comprendre au lecteur les conséquences de ce modr de développement. Il me semble au contraire plus opprortun d'y ajouter des considérations d'une certaine importance. 
Dans les pages précédentes le ce chapitre, je n’ai considéré

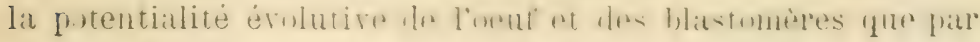
rapport a la nature des blastoméres qu'ils sont capables de

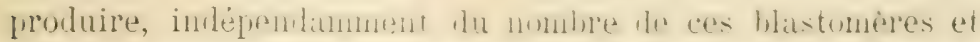

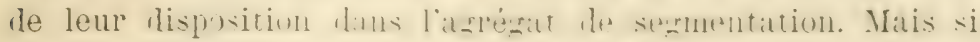
nous tenons compte de ces conditions aussi, nous en verrons ressortir des conséquences qui ne seront pas sans im-

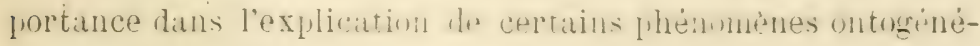
tiques.

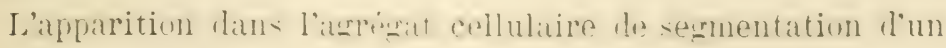
blastomère de la série $b^{\prime}, c^{\prime} \ldots$... l' est naturellement la cause de la formation plus ou moins tardire d'une différenciation

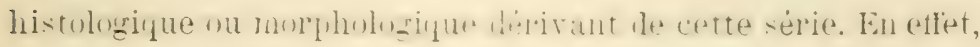
dans notre cas, le blastomère $\zeta^{\prime}$, en présence d'un milieu interne favorable, pourra donner lieu à une autre lignée de cellules, dont la nature sera déterminée par la constitution du bioplasma de $y^{\prime}$ et du milieu interne dans lequel celui-ci puisera ses substances nomprisinter, tont comme nous laarous démontré au chapitre XII.

Or, nous pourons fircilenent constitter que, dans le lérelnp-

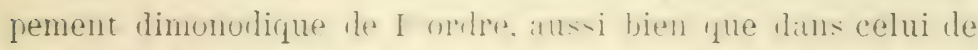

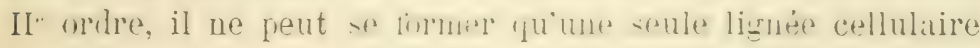
de la série que nous considérons; tandis que, dans. le développenent dimmolique lo Ill milre il sen tome deux, dans celui de IVe ordre, trois; dans celui de To ordre, cinq; et huit dans celui de Tre ordre.

Or si, dans l'agreat cellubaire. Im limesestatent absolument

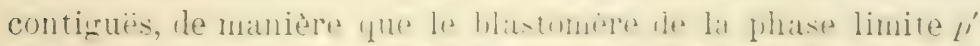

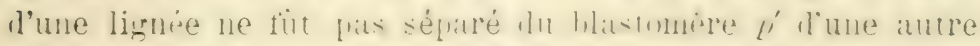

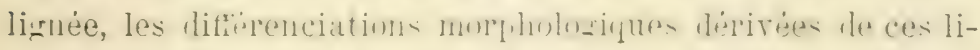

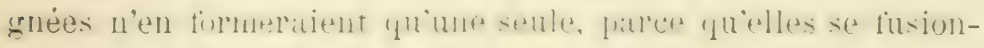
neraient en une différenciation unique. Mais si, ce qui est

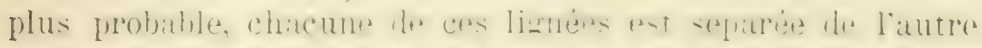

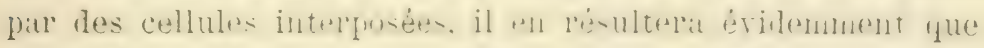

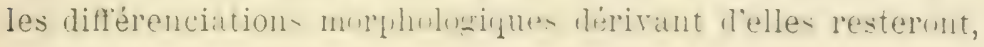


elles aussi, nettement séparées, et que leur nombre correspondra nécessairement au nombre meme des lignées cellulaires caractéristiques des divers ordres du développenent dimonodique.

Par conséquent, le dévoloppement les blastomeres isolés, alor's mème qu'ils possèdent la même potentialité érolutive que l'oeuf au point de vue qualitatif, ne pourra donner lieu, au point de rue quantitatif, à des résultats identiques à ceux du développement de l'oeuf entier.

supposons, pour mieux concréter nos idées, que le blastomère $p p^{\prime}$ soit le point de départ de la formation, plus ou moins tardive, d'une bande ciliée sur l'embryon. Evidemment, nous rerrons apparaître sur l'embryon dérivé de l'oeuf entier' une, leux, trois, cing ou huit bandes ciliées, suivant l'ordre du déreloppement dimonodique que nous supposons. Mais si, dins le déreloppement dimonodique de $\mathrm{III}^{\mathrm{e}}$ ordre, nous isolons les deux premier's blastomeres, chacun de ceux-ci, tout en étant capable de produire la différenciation histologique et morphologique caractérisée par' la bande ciliée, n'en produira pas deux, comme dans l'embryon normal, mais une seule.

Ie même, dans le déreloppement dimonodique de IV ordre, oì le nombre normal des bandos ciliéos serait de trois, un res deux premier's blastomeres, lo blastomère ", isolé, ne donnerait liou qu'ì deux bandes ciliées, et l'autre, c, qu'à un senle bande. De mème, des quatre premiers blastomeres isolés, trois donneront origine chacun à une bande ciliée, et un n'en proluira pas du tout.

Dans le développement dimonodique de $\mathrm{V}^{\mathrm{e}}$ ordre, où le nombre normal des bandes serait de cinc, le blastomère be en produirait trois, et le blastomere " deux seulement. Des quatre premiers blastomères isolés, trois en produiraient une seule, et un seul en produirait deux.

De mème, dans le developpement de VI" ordre, ou les bandes normales seraient huit, les deux premiers blastomères isolés en promluiraient, l'un cind, et l'autre trois; et les quatre pre- 
miers hastominess isolés prohluiraient l'un, trois bandes; deux, deux bandes chacun, et un, une bande seule.

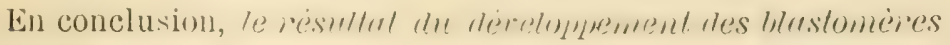
isolés, dans ce cas, est lel, qu'au point de vue quantilatif, la

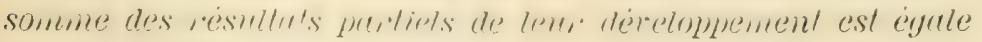
au résultat du dévelopnement de l'oeuf entier.

Je ne sais s'il existe des organismes présentant ces modes de déreloppement; mais je crois qu'il n'est pas tout-à-fait impossible que, dans l'immense rariété de la nature, il s'en trouve quelques-uns, dont le déreloppement ontogénétique puisse ètre expliqué par l'un ou l'autre de ces modes. Par exemple, la symétrie rayonnée secondaire des ichinodermes, dont le nombre des rayons est de 5, ne pourrait-elle pas trouver son explication dans le déreloppement dimonodique de r" ordre? Nous ne pouvons l'allirmer dès at présent d'une manière positive, vu l'insuffisance de nos connaissances sur les phénomènes intimes du développenent de l'échinolerme adulte aux dépens de la forme larvaire; mais nous ne pourons les nier a miori. Je n’insiste pas d'arantage sur cette opinion tout-i-fait personelle, d'autant plus que je ne connais pas de raisons plaidant pour ou contre : mais j'espere yue, par des expériences convenables on pourra obtenir des résultats capables, ou de la confirmer ou de la démentir.

De mème, par exmple, le léveloppenent des Cténophores peut être envisaré conme un déreloppement dimonodique de VI" ordre. La formation des huit bandes ciliées y trouverait son explication rationnelle. En ontre, les résultats expérimentaux du développement les blastomeres isoles, résultats qui paraissent itre hien différents de ceux qu'on obtient dans la plupart des autres animaux, correspondraient assez bien aux résultats théoriques que nous venons de démontrer.

Il est vrai que la coincidence entre ces résultats n'est pas parfaitenent exacte. Fn effet, dans les expériences, l'isolement des deux premiers bactomeres a domé pour résultat deux larres ayant chacune quatre handes ciliées; tandis que, d'après 
les resultats thémiques, ces handes derratent itre cine dans

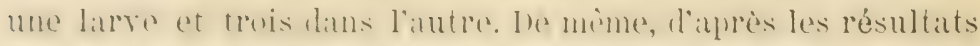
hes experienters (1), les quatle premiers blatstomeres isolés dument des latres à deux bindes ciliées seulement, tandis

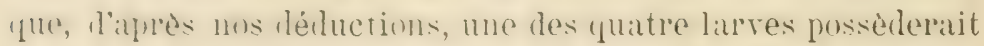
trois hamles ciliées, deux on pussèleraient deux, et une présenterait une banle senle. Mais je crois que ces expériences hirent itre répétées ef leurs résultats confirmés à noureau: car il n'y a pas, entre ces résultats obtenus par différents Iiolugistes cotte concombance rignureuse at laquelle on a le droit de prétendre dans les recherches scientifiques.

The concilence parlitite le nos résultats thériques arec cenx de lexp ipience peut itre constatee diuns ces larres que

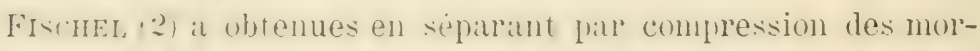
ceatux de l'agrégat cellulaire le segmentation de Beroe. Ici en effet, nous trourons une larre avec trois bandes, deux avec deux handes, et une arec une bande senle. Mais malheureusement, ces résultats ne peurent aroir qu'une importance secondaire: ear nous ne saroms pas duelle etait lat valeur morphologripue of la constitution des morceaux dont elles sont dérivées.

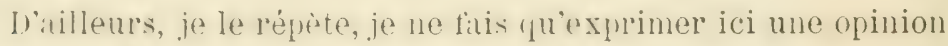
tont-it-litit fersomelle, et je ne reux pas insister darantage. l'ent-itre le mole le dérelnpprment des cténophores est-il, lui ansi, exchusirement monolique, comme dans la plupart des antres animanx, et les résultats diflérents qu'on ohtient par le hiseluppenent de leurs hastmueres ícolés ont-ils pour canse

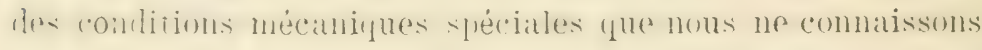
pas encore, mais que nous pourrions coinaître en examinant Hus rignmeusement et plus minuieusement le déreloppement ontogénétique de ces animaux.

(1) Drmescit H. and Morgan T. H. - Zur Anulysis dev ersten Entuickelungastadien des Ctenophoreneies, in: Arch. f. Eutwickelungsmech. II Bd., 1895, pp. 204-215. - Frscrel A. - Experimentelle Untersuchungen am Clenophorenei, in: Arch. f. Entwickolungsmech. VI Bd., 1897, pp. 109-130.

(2) Érschel A. - Loc. cit. VII Bd., 1898, p. 31. 
SII.

\section{Les développements polymonodiques.}

Ln autre mode de léveloplement hétérosénétique que nous devons encore mentionner est celui que j'appellerai le hror-

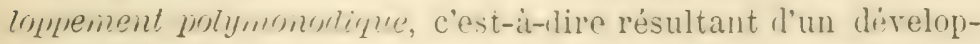
pement polvodique combiné arec un développenent monodique. On obtient ce mode de dérelnppenent lorsumon suppose, par exemple, que l'oeut suit jusiu ì un certain point le développement polyodique, et puis que chacun les hastomeres poursuit son déreloppenent suirant le mole monodique. Fous jourons en. avoir un exemple dans le schéma suivant:

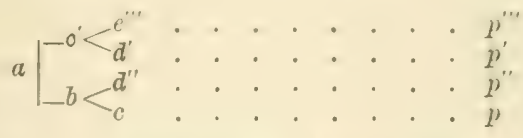

ou l'on roit que la potentialité érolutive le l'oufu, qui est simple dans le déreloppement monulique, et louble dans le dimonodique, devient qualruple ou multiple. Mais on comprend que, dans ce cas, la potentialité érolutire des blastomères dérivés du développement polrodinue ne pent ètre érale il celle de l'oeuf.

J'irnore si ce mode de déreloppenent se troure réalisé dans quelques organismes.

\section{§ III.}

Les développements cycliques.

Enfin, pour complitor l'examen le tomtes les combluationn possibles du développenent hétérugétique, il nous reste it

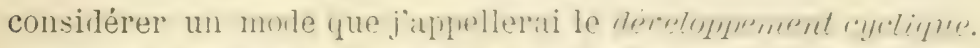

Celui-ci it lieu lor:que nous supposons qu'une des cellules dérivant du déreloppenent monolique, arrivée à la phatse 
pénuliène de l'érolution, se divise on dexux cellules, dont l'une représentant la phase limite, et dont l'autre est égale, par sa constitution hioplatsmatique, it lil memiere cellule, souche de tout le déreloppement monodique.

Ansi, par exemple, nous pourons supposer que l'oeuf $a$, en surant son drolution, arrire justu'it la phase o, et qu'alor's il puisse se diviser en deux bastomeres, dont l'un, l', phase limite, et l'autre, a, c’est-ì-lire égal à l'oeuf. Il est érident que dans ce cas, si cette cellule possédait en elle-même toutes les conditions intrinsedues constituant le milien interne de l'oeuf, elle serait capable de suivre, dans les conditions physicochimiques farorables, la mème érolution que l'oeuf, c'est-ì-dire qu'elle serait un oeuf capahle de se divelopper saus fécondation préalable. Nous rerrons, dans une antre partie de ce trarail, si ce phenomene est nossible et dins quelles conditions. Je me contenterai de faire remaruuer en passant que ce phénomène, que jo riens de supposer, pour plus de simplicité, à la fin de l'érolution de l'neuf', c'est-it-lire après une seule lignée cellulaire, peut aroir lieu, et plus facilement, après plusieurs lignées cellulaires, et, par suite, ¿t une phase plus ou moins éloignẻe du développement ontogénẻtique.

Ia supposition que je riens de faire pour l'oeuf, nous pourons la faire pour une autre cellule, souche d'une lignée cellulaire quelconque dans l'organisme. Par exemple, nous pourons supposer que la cellule ó dérivée de $y$ puisse se diviser en $y^{\prime}$ et $l$ et, l'une manière générale, qu'une cellule $o^{n}$ dérirée de $l^{\prime \prime \prime}$ puisse so diviser en $l^{\prime \prime}$ ot $l^{m}$. On comprend que, dans tous ces cas, la cellule qui est égale i celle dont elle est dérivée en possélera aussi la potentialité érolutive et sera, par conséquent, capahle de donner lieu aux mêmes manifestations, pourvu, bien entendu, qu'elle se troure dans le même milieu interne.

On prourait peut-ître, far ce développement cyclique, expliquer lat tomation des serments du corps dans les animaux dont la semmentation est homogime. Mais ces explications ne peuvent itre données que si l'on exanine tout particuliè- 
rement les diflérents cils, or alor's seulement quon peut constater, sans aucun loute jusible, que les segments du corps sont absolument et rigumeusment irlentiques entre eux: ce qui, je crois, ne se rérifie pas très sourent.

J'ai roulu, dans ce chapitre, exproser tous les modes possibles do déreloppement, afin de demontrer que, tout en supposant le développenent héterogénétique, c'ost-d-dire le plus simple, on peut expliquer les liflérentes manifestations ontogénétiques sans recourir à les hypotheses spéciales, et sans almettre dans l'oeuf une préformation de l'organisme ou un anisotropisme quelconcue ayant le moindre rapport arec la constitution morphologique de linpanisme qui en dérive.

C'est aux Biologistes mintenant d'examiner arec la plus rigoureuse attention, et en tenint conpte des moindres particularités, le déreloppement untugénétique des divers animaux et d'y appliquer l'un ou l'autre de ces moles de développement que je viens de décrire. chaque déreloppenent ontogénétique constitue à lui seul un problime yu’il liat résoudre indépendamment des autres. Les divers modes de déveloplement que je riens de mentionner ne doivent servir qu'à indiquer les interprétations les plus aptes a la solution des problèmes. Je laisse aux Biologistes la tiche de les modifier ou de les dérelopper conrenablement, ne provant éridemment, moi-même, dans un travail général, considérer tnutes les différentes questions que peut nous présenter la nature. 


\section{Chapitre XV.}

\section{La régénération.}

SOMMAIRE : Les conditions nícessaires de la régénération - Les limites de la rẻgénération - Le nombre énormo des cellules régénératrices - Le rapport numérique entre les différentes cellules de l'organisme - Les dimensions fixes des êtres - I' hétérogénéité des organes - Les rapports entre les cellules et le milieu interne - L'automatisme de la régénération - Son explication - La régénération physiologique et pathologique - L'origine des cellules régénérntrices et leurs rapports avec les feuillets embryonnaires Causes de l'impossibilité de la régénération - La régénóration morphologique ot ses conditions - Résumé.

C'est dans l'explication les intéressants phénomènes de la régénération, que nous pourrons apprécier mieux encore la bonte de notre interprétation et les arantages considérables du déreloppenent monodique: car nous rerrons que ces phénonènes ne sont qu'une conséquence directe et nécessaire de ce lérelnppenent même, et que, par suite, leur explication découle toute simple, claire et naturelle de l'interprétation que nous renons de lonner les phénomenes le l'ontogénèse. La régénération n'est en somme qu'un épisode du déreloppement ontogénétique.

Revenons au développement des blastomères isolés.

Fous arons vu et démontré qu'un des blastomères isolés, dans le cas du développement monodique, est capable de donner lieu a la formation d'un embryon complet, tout comme s'il itait rénni aux autres blastomères. I'où dérive cette faculté?

Arant trut, du morle de développement monodique que nous arons posé comme bitse de notre interprétation. En deuxieme lien, de la contitution hioplasmatique du blastomère. En troisime lien, des comditions dentoplasmatiques, physico-chimiques et mécaniques du blastomère même. 
C'est en eflet dins le léreloppenent monodique que tout blastomère possede la meme potentialité érolutive plue l'ueuf. Cette faculté est une consinquence directe et inéritahlo du mode mème de ce dérelongenent. Matis si tout hlastomere lat possède, cela est dir it sa constitution bioplasmatique, représentant une des phases de lorvhlution de l'oenf: car il est érident que s'il n'en était pas ainsi, le dérelopuement du blastomère isolé ne pourrait conduire anx mèmes résuitats que le déreloppement de liveuf. Il est clair encore que si les substances deutoplasmatiques n'étaient pas de la mine constitution que celles de l'oeuf entier, l'érolution du blastomère ne pourrait être la meme, et que si les comlitions physinues et chimiques du milieu n’étaient jats fitrorahles, le développement ne serait pas possible. Il en est le mime des conditions mécaniques. Si le blatonère isolé wnue lieu, far sa segmentation, à la formation d'une blastula sphériugue, tout comme l'oeut entier, c'est que les conditions micaniques dans lesquelles il se déreloppe sont égales à celles lans lespuelles se développrait l'oeuf. Yous sarons que les hlastomères isoles, n’étant plus arlhérents entre eux, acquirrent généralenent une forme sphérique, comme la plupart des oenfs dont il dirirant. (quoi donc de plus naturel que leur sermentations se poursuire de la mime

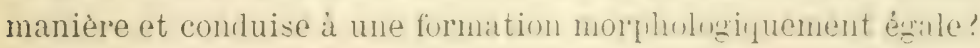

Cependant nous ne pourons dire qu'il y a, dans tous ces cas, une réritable régenération et, noins encore, une postregénération. Lorsqu'on isole. par exemple. les leux blastomeres 1", ", chacun de ceux-ci peut momer migine it un embrton entier. Or, le blastomère 7, en se dirisant it son tour en c, ", produit par sa dirision un autre blastonere 'égal a celui yqui en ariat

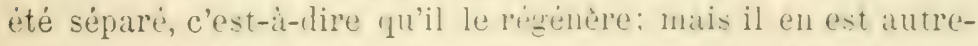
ment du blastomère ": carr crelui-ci, en se divisant en ", ", ne regénere fas le hastumere l, qui arat été sepraré. Et pourtant. les résultats du léveloplenent sont particitenent éraux fun tous les deux.

Suprosuns maintenint que, dans un agregat cellulitire de 
segmentation, on exporte un blastomere quelconque, par exemple, le blastomère /' de la phase limite. Qu'en dérivera-t-il?

Evilemment, un antre blastomère, égal à celui qui a été exporté, prendra bientot origine par la division continue des autres blastomères qui se trourent dans les phases précédentes de l'érolution de l'oeuf, et le déreloppement se poursuivra normalement, tout comme si l'exportation n'arait pas été faite. Et si l'on reut répéter l'opération, les résultats en seront toujour's les mêmes, d'autres blastomères $p$ se produisant successivement en substitution du blastomere exporté.

Dans ce cas, il y a une véritable régénération; mais celle-ci, comme chacun peut le constater, n'est pas une faculté spéciale de l'or'ganisme, mais une conséquence inévitable du mode même du développement.

Jusqu'à quelles limites pourra se faire cette régénération? Evidemment, tant que l'agrégat cellulaire possède des blastomères á des phases précédant la phase $p$, la régénération de celui-ci sera toujours possible, pourvu, bien entendu, que les substances deutoplasmatiques nourrissantes et contenues dans les blastomères soient en quantité suffisante.

Supposons que les phases de l'érolution de l'oeuf soient 1.4, atinsi que nous l'avons fait dans les exemples précédents. On pourra facilement remarquer que ce nombre n'est pas grand et que, dans la réalité des faits, les phases évolutives que l'oeuf loit franchir pour arriver à la phase limite sont peut être sourent plus nombreuses. En les supposant tout simplement 1.t, la phase limite $p$ sera atteinte après la $7^{\prime \prime}$ division.

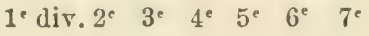

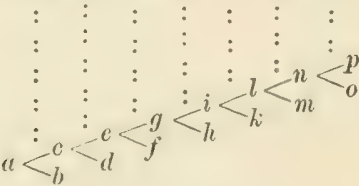

Mais pendant que cette érolution s'accomplit, il s'est produit, far los divisions répétées, fi blastomères à des phases 
différentes de l'évolution. Or, chacun de ces blastomères $b, a, r$. $h, k$, in poursuirra son érolution rel's la philse linite $l$, qu'il atteindra apres un certain nomlne le divisions lependant de la distance qui le sépare le cette phase, et, par suite, chanue division donnera lien i l'autres hlastomeres, qui, a leur tour, se diviseront et poursuiront, (2ux atusil, lem évolution.

Ainsi $n$ se divisera en $n$, ", et fuls "se traslomera en $\eta$ et $n$ se divisera en ", 1 , dont "se transformer"i, lui anssi, en $\mu$ :

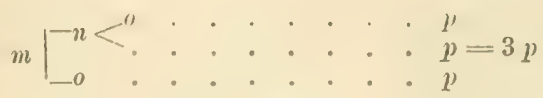

c'est-à-dire que les blastomères $p$ dérivés de $m$ seront 3 .

De même $k$ se divisera en $l, m$ et $l$, à son tour, en $m$, $n$; et comme nous renons de voir que "In peut donner origine a 31 " et $n$ a $2 p$, le nombre des blastomères $p$ dérivant de $k$ sera égal à la somme des blastomères dérivant de $l$ et $m$.

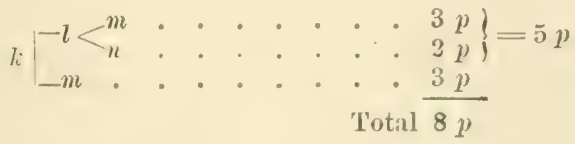

En appliquant le meine raisunnement anx antres blastomeres, on obtiendra: pour

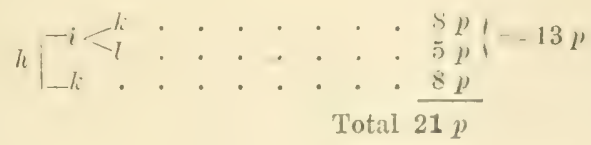

pour:

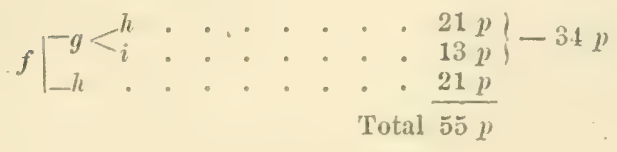

pour:

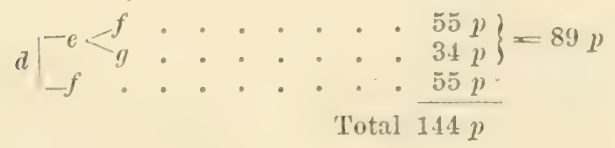


et enfin pour:

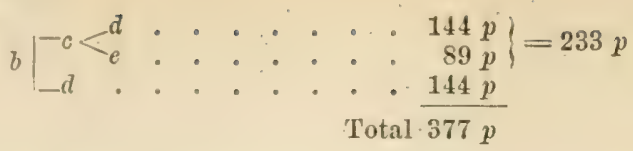

Or, si nous faisons la somme de tous les blastomeres pl pouvant dériver de la seymentation de ces différents blastomères, nous arons:

$$
\begin{gathered}
b=377 p \\
d=144 p \\
f=55 p \\
h=21 p \\
k=8 p \\
m=3 p \\
n=2 p \\
\text { Total } 610 p
\end{gathered}
$$

c'est-it-lire que l'oeuf, par sa segmentation, suirant le déreloppement monodique, peut donner lieu ì 610 p), et, par conséquent, on pourrait enlerer 609 fois le blastomère $p$, que celui-ci se régénérerait toujours.

Or, si nous comparons entre eux les nombres des blastomères y' que l'oeuf et chacun des blastomères sont capables de produire, nous obtiendrons;

$$
\begin{array}{lllllccccccccc}
0 & n & m & l & k & i & l & 9 & f & e & l & c & b & a \\
1 & 1 & 1 & 1 & 1 & 1 & \mid & \mid & 1 & 1 & \mid & \mid & \mid & 1 \\
1 & 2 & 3 & 5 & 8 & 13 & 21 & 34 & 55 & 89 & 144 & 233 & 377 & 610
\end{array}
$$

ou l'on roit quils forment une serie (1) dans laquelle chaque terue est egal à la somme des deux termes précedents, et que le dernier terme est égal à la somme des termes précédents alternativement considerés, augmentée d'une unité.

Il faut renarquer que ce nombre consilérable de 610, que nous arons obtenu dans ce cas en supposant seulement 1.1 phases de l'évolution de l'oeuf, s'accroit rapidement si l'on supprose que les phases soient plus nombreuses. Par exemple

(1) Cette succession de termes est connue par les mathematiciens sous la dénomination de série de Fibonacci. 
si, au lieu de 1 t, nous : uppusinns li phases de l'érolution de l'oeuf, nou-obtiemurions 2,st blastomeres de la phase linite.

Yous arous ru que le blastomère de lat phase linite de l'érolution de l'oeur derient le fuint de léprart le la ? lignée cellulaire, caracteristique de la 2 phase de lontugériese. or, si l'on suppose, four phu- de simplicite. que lat phase limite de cette 2." lignée cellulaire soit li et, far suite, séfráe de la phase I' par 14 phases internemiates, on comprendra facilement, en $y^{r}$ appliquant le mine raismnement que nous renons de faire pour l'oeut, que chaque hitstumère ${ }^{\prime}$ pourra, par sal nourelle érolution, prorluire lill pi. Et si tums les blastomères p) pourant dériver de loenf suiraient la méme érolution, on

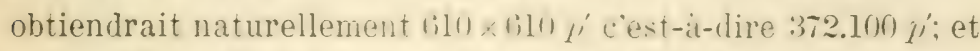
par conséquent, si l'on purait enlever te l'agrérat cellulaire toutes les cellules $f^{\prime}$ a mesure qu elles apparaisent, nn obtiendrait 372.100 fois la régénération de cette cellule.

Mais comme chaque cellule fi freut levenir le print de départ d'une autre lignée cellulaire $y^{\prime} \ldots . . . j \prime$, par exemple; comme, d'autre part, chaque cellule f' peut, four les raisons émoncées, donner origine it "illl f". le nombre total de ces cellules pourra devenir $610 \times 372.100=226.981 .000$.

Noús royons donc quen laisant dess suppositions assurément plus simples que les phenomines reel-on arrive it des rénutats raiment extrondinares far rabnort au nombre des cellules capables de se régénérer.

Il est rrai que, dan- le riveloflenent des organismes, les blastomères ${ }^{\prime}$ ne surent pa- tous lirvolution de la deuxiène lignée cellulaire: car une fratie de ce- blastoneres suirront une autre érolution. caractéristinue des liornées cellulaires bilatérales, dans le cas de la smetrie hilatrirale, ou des autres lignées radiaires, lans le cits le lit rmétrie rayonnée; mais, meme en supporant que le nombre en soit plus petit, il ast néammoins érident quapres une certain nombre de lignées cellulaires, les cellules hérives de $f^{\prime}$ seront en nombre vaidment extraordinaire. 
Or, si nous considérons que les organes de la plupart des organismes qui ont achevé leur déreloppement ontogrénétique, sont tormés des cellules issues originairement de l'veuf, mais en pilssant far une série flus ou moins longue de lignées cellulaires s'étant succédées l'une al l'antre, nous pourons nous convaincre que lo nombre des cellules de ces organes peut être énorme.

si donc ces cellules arrivient toutes, absolument toutes, at la phase limite caractéristique de leur dernière érolution, c'esti-dire à la différenciation hi-tologique caractérisant l'organe que nous considérons, il serait évident qu'à part les dimensions que l'organe acquerrait, la régénération de ces cellules et, par suite, de l'organe, après l'exportation, ne serait plus possible. Nous renons de roir en effet que la régénération n'est possible qu'en tant qu'il existe des cellules dans quelques-unes des phases précérlant la phase limite et, par suite, capables d'arriver it leur tour a cette phase. Mais si toutes les cellules sont arrirées à la phase limite, évidemment il n'y en a plus d'autres à des phases précédentes, et la régénération devient nécessairement impossible.

Mais cette transformation totale des cellules d'un organe quelconque est-elle un phénomène réel? Je ne le crois pas, et je vais essayer de le démontrer.

Considérons donc un organe quelconque 0 , et supposons que la différenciation histologique, que j'indiquerai par yo, soit caractéristique de cet organe.

Pour les raisons que nous renons d'énoncer, les cellules prorenant des nombreuses lignées précédentes et capables d'arriver a la phase flo en passant par des phases que nous supposerons $\eta_{0}, c_{0}, 17_{0} \ldots .$. , seront au nombre de plusieurs milliards et, parfois mème, de plusieur's milliards de milliards.

Ie tontes ces cellules, plusieur's millions on plusieurs milliards arriveront successivement i la phase po, et représentrront autant d'élenents histologiques carractiristiques de l'organe que nous considérons. Nais pendant que le nombre 
de ces éléments ra s'accroissant, d'autres millions ou milliards de cellules se trourent encore dans des phases de leur évolution antérieures à $p_{0}$.

A ce moment, l'organe sera donc constitué de plusieur's milliards de ces cellules a la phase /o et de plusieurs milliards de cellules à d'autres phases $\iota_{0}, m_{0}, l_{0} \ldots$. Si donc l'érolution des cellules s'arrêtait a cet instant, on comprend facilement que la constitution de tout oryane ne serait pas parfaitement homogène, c'est-à-dire que ses cellules ne possèderaient pas toutes une différenciation histologique absolument identique.

Or, je crois que, dans la nature, les choses se passent réellement ce cette manière.

J'ai dit ailleurs que nous derons considérer tout organisme comme un système symbiotique très complexe, ou les cellules en représentent les unités constituantes, et que l'entretien de la vie de l'organisme entier dépend de l'entretien de la vie de toutes les cellules. Or, la vie de celles-ci n'est possible qu'en tant qu'elles s'entr'aident réciproquement, c'est-à-dire en tant que les substances sécrétées par les unes peuvent servir de nourriture aux antres.

Il s'ensuit que la quantité de ces substances doit être nécessairement limitée dans le nilieu interne de l'organisme, et que cette limitation est léterminée par un rapport quelque peu fixe entre les cellules mêmes de différente nature.

Supposons en effet que les direrses espèces cellulaires constituant l'organisme soient indiquées frar $A, B, C, D, E, F, G$, et que les substances sécrétées par 1 constituent la nourriture des cellules $F$.

Il est alor's érident que le nombre des cellules $F$ possibles dans l'organisme sera subordonné an nombre des cellules A; et si les substances sécrétées par $F$ serrent de nourriture aux cellules $C$, le nombre de celles-ci dépendra nécessiarement du nombre des cellules $F$ directement, et indirectement du nombre des cellules $A$.

Par un raisonnement analogue, si l'on étend aux autres 
cellules des relations symbiotiques semblables, on comprend facilement qu'il loit s'établir entre toutes les diverses cellules de lorganisme un rapport numérique constituant un équilibre symbiotique, non seulement au point de rue de la nature des unites symbiotiques, mais aussi au point de rue du nombre de ces unités.

Or, nous sarons que le rolume total de l'organisme dépend de la somme des rolumes des cellules et des substances intercellulaires qui les composent. Il est rrai que les rolumes des cellules peurent rarier d'une espece a l'autre, et dans les divers individus d'une mème espèce, ainsi que le volume des substances intercellulaires: mais nous ne pourons nier que le facteur principal du rolume total de l'organisme soit, arant tout et surtout, le nombre de ses cellules. Nous pourons donc comprendre comment et pourquoi les dimensions des organismes sont, dans certaines limites bien entendu, fixes pour chaque espece. La limitation du rolume total pour chaque espèce d'êtres ne serait donc qu' une conséquence directe, inévitable de sa constitution.

D'autre part, comme toute la vie de l'organisme n'est qu'une série continuelle de systèmes symbiotiques différents, se succédant du commencement de l'ontogénèse jusquu'à la mort, où, par conséquent, ce rapport numérique des éléments doit toujours se maintenir, nous pourons comprendre aussi comment le nombre des cellules des systèmes strmbiotiques des premières phases de l'ontorénèse peut influer sur le nombre des cellules des systèmes symbiotiques des autres phases de la rie, quind nême celles-ci sont très éloignées des premières phases du développement.

Si donc on accepte le principe de ce rapport numérique tel qüe je riens de le démontrer, il est naturel que les cellules, hien que possélant en elles-mèmes la potentialité d'arriver à une certaine phase rérolution, ne pourront néanmoins y arriver toutes: car nous sarons quelles ne peurent atteindre laur but que par les divisions réuetées et que ces dirisions 
exigent une périule préalihle d'ilsinilation. Or, si les suhstances néressaines four l'assinilation manquent. celle-ci ne peut se faire et lovolution ultériene en est nécessairenent empèchée.

Il dérive, comme conséquence naturelle de ce que je viens de dire, que rout orranisne et, grar suite, tout orerane résulte composé de plu-ieurs aspees de cellules, dont quelquesunes à une phate didrolution lat plus arancée, et les autres at les phases moins atrancés de la mine érolutions et, pour levenir a l'exemple cité, que l'orsane ") résultera composé de cellules a lia phatse pon d'atutres cellules a des phases $n_{0}, m_{0}, l_{0}, k_{0} \ldots$.

Les organes ne seraient lone frim hommènes lans leur consti-

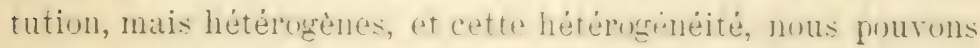

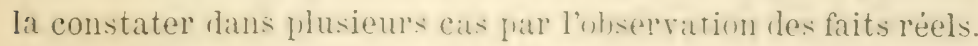

On sait que les cellules lu tuhe intretinal ne sont pas toutes parlaitement ilentiques, menes dins nue petite portion de ce tuhe. Fous pourons en lire autant des cellules rénales et de plusieurs autres organes, funique cette diversite ne puisse parfois ressortir arec la plur wrame érilence, taute de morens suffisants d'inrestigation.

Il fut remarquel cepenhiut que, hien que certaines cellules n'arrivent pas à la phase linite le leur putentialité érolutive, celat n'exclut par quelles puisent jouler un certain mile dans la physiologie de l'organe.

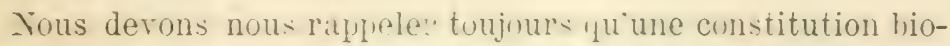
plasmatique, quelle quelle soit, rerrésente en tout cas une differenciation histologinue déterminée. I'all conséquent, ces cellules, ayont une constitution hinplasuatique propre, fossèrent, elles atusi, une diférenciation hi-tologique caractéri-

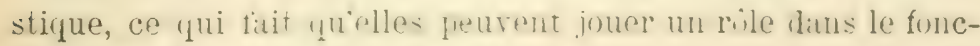
tionnement général du corps.

Tout celat itant pos. Iat rénération des ormanes derient un phenomene le la plus sranke simplicitie et tont-it-fitit naturel: car il est évilent que, si l'on exprerte dे l'organe o, par 
exemple, les cellules /'o, d'autres collules égales, dérivant de la division des cellules aux phases antérieures: $n_{0}, m_{0} \ldots .$. , pourront les remplacer.

Il en est ile mine si nous supposons qu'on exporte les cellules 110. ino: carl d'autres cellules de la mème nature, dérivant des phatses antérieures $l_{0}, k_{0}, i_{\omega}$, lus remplaceront aussitot. Cette substitution sera naturellement possible, tant qu'il $y$ aura Mans l'ororanisme dos cellules à des phases antérieures de l'érolution caractéristique des cellules exportées.

Mais si l'exportation de l'or'rane a lieu de telle sorte que non seulement une catégrorie de cellules, mais toutes les cellules pourant suirre l'érolution yui a ploduit l'organe, soient enlevées de l'organisme, la régénération ne pourua plus absolument se faire.

Supposons, par exemple, que dans l'organe o il $y$ ait des cellules à toutes les phases de l'érolution $b_{0}, c_{0} \ldots \mu^{\prime}$, et que dans loorganisme il n'existe plus de cellules dont celles-ci sont dérivées. Tant que l'on exporte une partie seulement de l'ororane, celle-ci pourra se régénérer aux dépens des autres cellules qui restent; mais. less quon fera l'exportation de tout l'organe, la régénération ne pourra plus avoir lieu.

Mais, moljectera-t-on, pourquoi les cellules qui ne sont pas encore arrirées à la phase limite de leur érolution n'y arrivent-elles que lorsque une partie de l'organe a été exporté? Pourquoi, par exemple, en supposant l'organe $O$ constitué de cellules $1{ }^{\circ}, n_{0}, m_{0}$, etc., les cellules $i_{0}, n_{0}$ n'arrivent-elles à leur tour d̀ la phase 1'o yu'après l'exportation de toutes ou d'une partie au moins les cellules to? Quel est, me dira-t-on, lo stiinulus qui provoque, après l'exportation, la progression des cellules dans leur évolution spéciale?

C'est ce qu'on peut comprendro tres facilement; car cette manifestation du merreilleux automatisme qui nous frappe rans les phénonènes biologiques n'est, en dernière analyse, qu’une simple conséquence naturelle du mode de son rléveloppement et du principe même de sa constitution. 


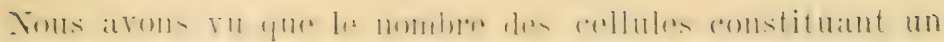

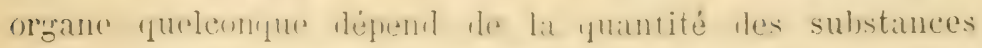

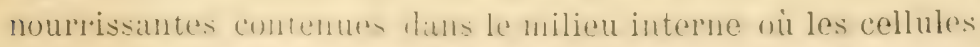

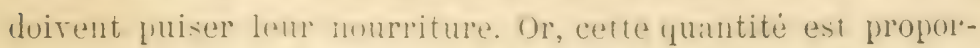
tionnelle an nombro dre callules yuelle loit nourrir, et si elle saccroit, le nombm des collules yni sen nomersent pourlat amgmenter anssi: tambiz gur, si mlle diminue, la rie de celles-ci ne pourral plus s'entretenir nomialement ot, naturellement, elles périront, en partio an muns, finte l'alimentation. P'ar conséfuent, la fonction de l’ormune, fui résulte de la vie de toutes ses cellules, en subirit les perturhations blus ou moins fortes.

Certe manière l'interprotur l'oreanisme nums permet aussi l'expliquer ces rapports tros interessints el meore peu connus rntre les diver's organes, yur num- alprelons les phénomines de corrélation.

En eflet, comme los substances nomplissutes des cellules provienuent de lat sécrétim l'autlexs cellules de l'organisme mème, si l'ou suppose que, pour une cause quelconque, normale on anormale, lat quantité les substances sierétées flar certaines cellules aumentr, lo mombe lles cellules qui se mourrissent aux dépens de ces substances doit auguenter aussi. D'oi il résulte que l'accroisement de certains organes est une conséfuence rirrecte de l'accroisement d'autres or granes.

Certes, si l'on punrait connaitre, parmi les nombreuses substances chimiques composint le milieu interne de l'organisun, quelles sont celles yni serrent il lat mutrion les cellules d'un organe détermine, et le yuelles antros cellules dérivent ces

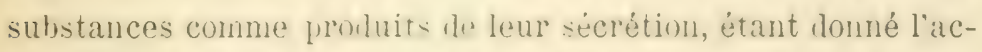
croissement l'un murane, muns pumrions en comnatre la cause, et la retrourer dans l'acemissement dra linsane dunt dérivent ses substances nomrussantes. Mats, malhourensement, cela n'est pas toujours gos-ililu dius l'atat actuel de la science.

C'est peut-itre dins certe solte de riplorts aussi gu'il faut 
rechereher lorigine de ces proliférations cellulaires pathologiques dont la cause est jusqu'ici inconnue.

Comme conséquence réciproque de ce que nous venons d'énoncer, il s'ensuit que si, par un procédé quelconque, par exemple, par l'exportation d'une portion d'organe, nous diminuons le nombre des reliules de celui-ci, les substances nourrissantes du milieu interre, dont la quantité n'a pas été diminuée, seront proportionmellement plus abondantes, ce qui rompra l'équilibre symbiotique. Si donc, dans l'organisme, il existe encore les cellules capables de suivre leur érolution caractéristique, ainsi que je viens de le démontrer, celles-ci se diviseront ultérieurement, et leur prolifération, tandis qu'elle rérrénèrera d'autres cellules à une phase d'évolution égale it celle qu'araient les cellules exportées, en augmentera aussi le nombre. De cette manière, on arrivera peu ì pen à la reconstitution de l'écuilibre normal. Celui-ci atteint, la prolifélation cessera, et l'organisme se retrouvera daus ses conditions normales primitives.

La régénération se fait donc automatiquenent; mais cet automatisme est un phénomène naturel, qui ne dépend nullement de facultés spéciales plus ou moins téléologiques ou providentielles de l'organisme, mais du mode même de son développement et des principes de sa constitution.

I)'aillours, la régénération accidentelle ou pathologique n'est, en dernière analyse, qu'un phénomène analogue à la régénération régulière ou physiologique.

La chute de certains éléments histologiques et le remplacement de ceux-ci par d'autres éléments, c'est lì un phénomène qui s'accomplit normalement dans beaucoup d'organismes. Je ne citerai que les faits bien connus de la régénération régulirre et physiologrique des cellules de l'épiderme, des poils, des plumes, etc.: ainsi que de la muquense utérine chez les Mammiferes a carduque, des cellules de l'intestin de certains insectes, des cellules du sang chez la femme, des poils et des cellules de la coifle de la racine dans les végétaux etc. 
Dans tous ces cas, lo phénomene se rend manifeste it nos moyens dobservation, nu bien parce yulil s'accomplit périodiquement, ou bien parce que les éléments qui se renouvellent sont abondants, ou bien encore parce que la chute et le renouvellement ont lieu dans des parties du corps oil l'observation est plus facile et commode. Mais je crois ot je suis convaincu que des phénomènes analogues s'accomplissent dans tous les organes et dans tous les orinamismes, hien que, dans la plupart des cas, ils échappent à nos recherches, ou que la constatation en soit plus diflicile.

Si l'on suppose, par exemple, que, lans un organe quelconque, les cellules tombent et so récrénèrent en nombre très petit, le phénoméne peut bien passel absolument inapercu; et pourtant, il est érident que si lat chute ef la régénération sont continnes, elles peuvent aboutir, dans ce cas, à un renouvellement de toutes les cellules de l'organe, aussi bien que dans les cas précédents.

Le renouvellement des feuilles est un phénomène qui ne se vérifie pas moins dans les ambres ì fenilles dites persistantes que dans les autres à feuilles caluques, bien que, dans ces derniers, il nous paraisse beancoup plus érident. Cela est dû naturellement a ce fait, que lans les arhres à feuilles carluques, celles-ci tombent et se régrnèrent presque simultanément, tandis que dans les autres, la chute et le renourellement n'ont lieu que graduellement et peu à peu.

Cet exemple a uniquement prour but de démontrer quo nos connaissances sur les régénérations physiologiques des collules des organes sont tout-i-fait suhorionnées au mode niême dont ce phénomène s'accomplit et que, par suite, celui-ci peut hien avoir lieu sans qu'il soit possible, ou du moins facile, de s'en apercevoir.

Or, je crois que la durée de la vie des cellules n'est pas égale à la durée de la vie dr l'organisme même qu'elles constituent. Elle est, au contraire, hien plus courte et ses limites sont dépendantes de la nature des celiules. Mais quelles que 
soient cos limites, il s'ensuit nécessairement que toutes les cellules doirent mourir, ce qui lait que d'autres doivent les remplacer it mesure qu'elles pririssent. A ce point de vue, l'organnisme est une succession continuelle de générations cellulaires se remplaçant sans cesse dans leurs fonctions.

Peut-itre sont-ce précisément ces cellules que les phagocytes digèrent it mesure qu'elles meurent. S'il en est ainsi, et j'en suis convaincu, dans la prétendue lutte des éléments histologiques, les phagocytes ne tueraient que des cellules mortes, et leur importante action phagocytaire serait tont-a-fait subordonnée it la mort préalable des cellules qu'ils doivent détruire.

Quoi qu’il en soit, entre la régénération physiolngique et la régénération accidentelle il n'y anrait done pas, d'après mon interpretation, une diflérence fonlanentale; car tontes les deux se feraient de la mème manière. Cependant, la régénération accidentelle se rend plus manifeste, parce que les cellules exportées tout d'un coup étant plus nombreuses et formant, par suite, une masse remarquable, leur remplacenent frappe plus vivement notre attention.

Quant à l'origine des éléments noureaux destinés à renplacer les autres qui ont disparu, nous pouvons la déterminer assez exactement l'après notre interprétation, pourru, bien entenrlu, que nous connaissions l'origrine ontogénétique des éléments en question.

Yous sarons que, dans un organisme pluricellulaire, tous les éléments histologiques dérivent nécessairement d'une seule cellule, l'oeuf, par une série plus ou moins longue mais ininterrompue de lignées cellulaires. si, par suite, nous considérons un organe queleonque, par exemple, l'organe (), que nous supposons formé de cellules $\eta_{0}, n_{0}, m_{0}$, etc... constituant par leur ensenble toute une lignee cellulaire $h_{0}, c_{0} . . . \nu_{0}$, cette lignée sera dirrivée inévitahlement l'une lignée précédente, que nous pouvons indiquer par les phases $b_{n}, c_{n} \ldots p_{n}$, et celle-ci, à son tour, lune antre lignée précédente $4 m,(m \ldots l)$, de manière 


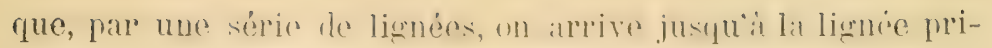
mitive $b, c \ldots l$, issue de la segmentation de l'oeuf.

Or, de même que, dans la constitution de l'organe, il peut exister, ainsi que je l'ai démontré, les cellules qui ne sont

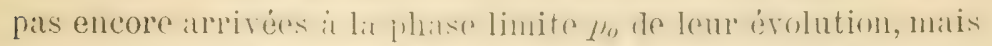

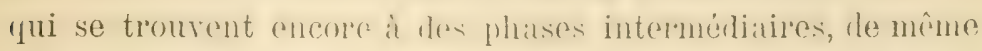
il est possible et mimer probable que les collules des lignées

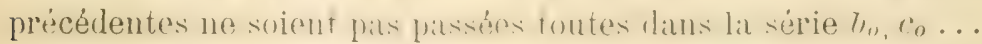
$p_{o}$, mais qu'il existe encore des cellules á des phases de la lignée précédente $b_{n}, c_{n} \ldots l n$. Et si nous étendons le même raisonnement aux autres lignées, nous pouvons prévoir qu'il n'est pas impossible de trourer. dians l'mpanisme, des cellules à des phases différentes des lignées $b_{m}, c_{m} \ldots p_{m}$, etc.

S'il en est ainsi, lorsqu'on enlève une partie des cellules de l'organe $O$, par exemple les cellules aux phases $p_{0}, n_{0} ; m_{0}, l_{0}$, on verra nécessairement que les cellules restantes $k_{0}, i_{0}, h_{0}$, etc. prolifèreront et donneront lieu à d'autres cellules qui arriveront successirenent aux phases descellules exportées. Nous constaterons done qur la régénération le lat partie exportée de l'organe aura lieli aux dépens des autres cellules de l'organe même.

Mais si l'on fait l'extirpation de tout l'organe, on enlevera de l'organisme toutes les cellules de la lignée $b_{0}, c_{0} \ldots p_{0}$, ce

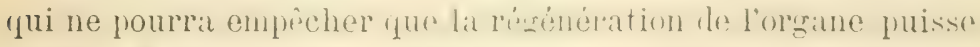

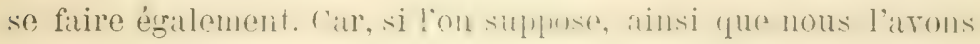
fait, qu'il existe encole, dans l'organisme, des cellules de la lignẻe $b_{n}, c_{n} \ldots p_{n}$, celles-ci, par des proliférations successires, arriveront peu ì peu à remplacer les cellules exportées. Dans ce cas, nous constaterons que la régénération n'a plus lieu aux dépens des cellules de l'organe même, puisque cellesci n'existent plus dans l'organisme, mais qu'elle ne se fait pas moins aux dépens de cellules qui n'appartiennent pas à l'organe qu'elles ont néanmoins le pouroir de régénérer.

Or, si les cellules de la lignée $b_{n}, c_{n} \ldots p_{n}$, constituent par lem ensemble un autre orane puelcompite $x$ de londividu 
yue nous considérons, ar qui ost parlatitement possible ot probable, nous reprons alor's la rézénération de lorgane o se faire aux dépens de l'organe $N$.

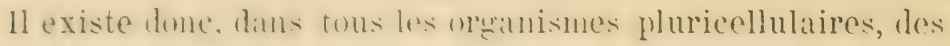

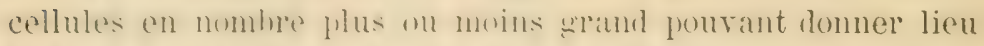
a la régénération hes parties exportés. ce sont ces cellules quon appelle fénéralement les cellules embryonnares indifférentes.

C"est néimmoins sur lat prétendue indifference le ces cellules qu'il fiut sontemdere exictenent. Cirr si l'on considere ces celInles au foint de vur de leur diflérenciation histologique, cette indillérence n'existe fats. Tous arons ru en eflet que, quelle que suit la constitution hioplasmatique des cellules, celle-ci

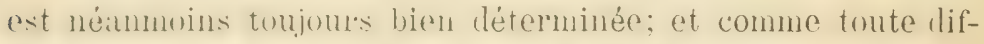

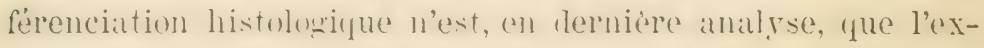
fression de cette constitution, il sensuit gu'me indillérence histolugique ne peut exister dins ancunte des cellules de l'organisme. L'oeuf même, qui, à ce point de rue, pourrait être envisitré comme le protutype des cellules indiffirentes, ne l'est. pas ahsolunent. Il possèle, lui anssi, une diflérenciation propre caractéristique.

Si, au contraire, nous consirlerons cette infifférence à un intre point le rue, c'est-id-lire en tant que ces cellules peurent dunner origrine it des éléments histologriquement différents entre eux, chla est raiment exact. Tous arons vu que l'oeuf, quniqu fus-èlant un diférenciation histologique bien déterminee, yaut néammins ponluire, en suirant son érolution caractipistique, des cellules histolngiquement différentes, de sorte plue la hastula peut itre comsidére comme un organisme it cellules hétérorines. Et pourtant, chacune de ces cellules leut, a sol tomr, produre l'autres cellules, elles aussi, dilfërentes.

Ainsi, pour revenir à notre exemple, si l'on exporte de l'organe $O$ les cellules aux phases $\eta_{0}, n_{0}, m_{0}, l_{0}$, et si l'on consiliere un whlule it lit phire hi., que nous supposerons restée 


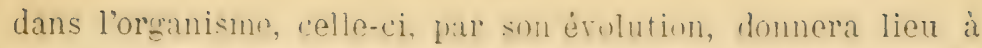

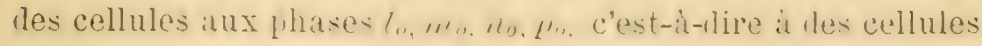
histologiquenent ditlepentes.

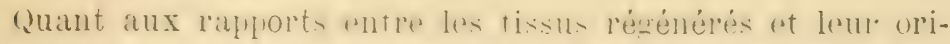

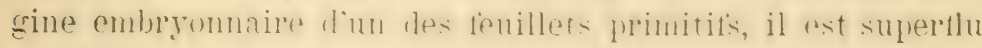

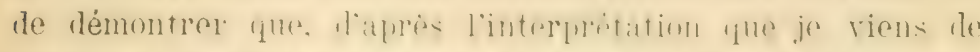

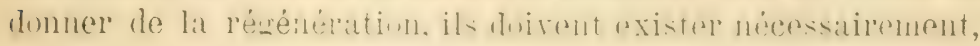
comme conséquence naturelle. La plupart des expériences plaident en effet en fareur de ces rapports; mais les résul-

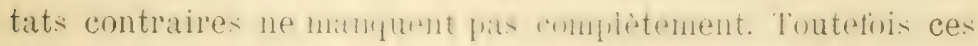

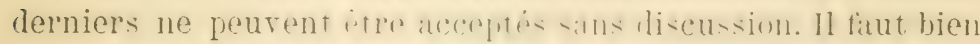
remaryuer, dans cette stre liexpériences, yüil pent se pré-

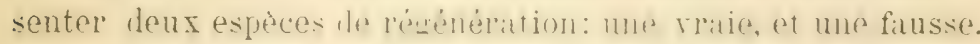

La régénération traie, que nous arons considérée jusqu'ici,

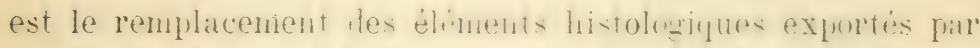
l'autres éléments histologinulunent émanx. Lal régénération

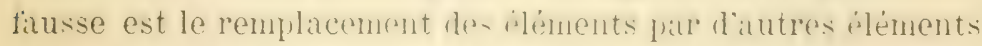

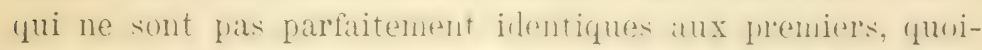
qu'ils puissent donner lien frar lour ensemblu is une formation morphologiquement semblable.

D'alpes notre interuretatim, la rézénération est lone une conséquence directe, naturelle at nitersithe du mole mene de déreloppenent que fai josé commur bats do lontogénèson,

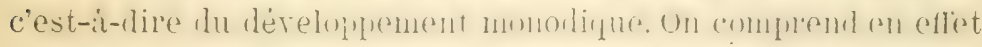

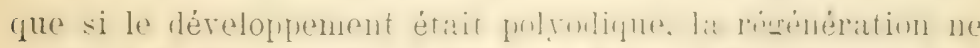
pourrait se faire absolument: atr un élément histoloritgue, une

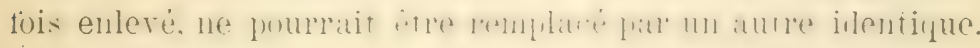
Ce remplacement n'est un phénomène possible que dans le

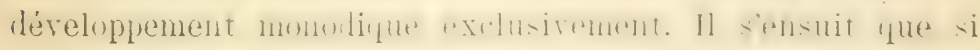

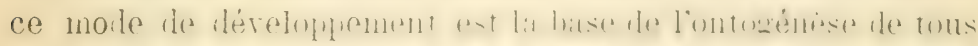

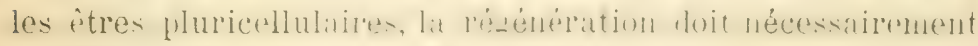
se vérifier dans tous ces organismes.

Mais, s'il en est ainsi, pourcquoi, me dira-t-on, la faculté de

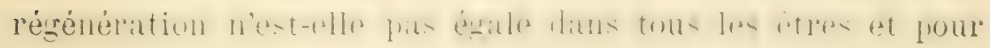
tous les organes? 
Revenons a lexamen attentif de toutes les conditions nécescaires pour la récenération. of mous y trourerons une réponse satisfaisante à cette objection.

Nous arons ru, au commencement de ce chapitre, que la rérinciation exige trois conditions: 10, la présence des cellules cirpables de suirre une erolution les amenant à une dillërenciation histologique égale à celle des éléments enlerés, c'est-it-line la présence des cellules qui, par leur constitution bioplatsmatique, représentent une des phases par lesquelles sont passés les éléments mèmes qu'elles doivent remplacer; $2^{\circ}$ ) un nilieu interne égal à celui lans lequel se trouraient les élénents histologriques exportés lors de leur evolution rers une diflérenciation déternume:e: 3 ) les conditions mécaniques égales i celles qui existaient lor de lat formation morphologique que lon consilere. On comprend facilement que si quelques-unes de ces conditions manquent, la régénération ne pourra plus aroir lieu ou bien ru'elle sera incomplete.

Rerenons, pour plus de sinpplicité, à l'exemple de l'oeuf.

Nous avons vu que la segmentation de celui-ci suivant le léveloplement monodique peut condure a la production de fill blastomeres a lat phase limite 1\%. Mais, a catuse du milieu interne de l'arreirat de segmentation, tous ces blastomères leviennent le point de lépart diantres lignées cellulaires, ćest-at-dire des lignnées nédianes et des lignées bilatérales. Lat surnentation acherée, il n'y aura donc, dans l'aurégat celIulaire, yne des cellules aplatentunt a l'une ou a l'autre de ces linés, fous les hastoneres p ayant disparl pour domer migne chacun à une des lignées susdites. Supposons donc fu'on puleve a cet agregat cellulaire toutes les cellules des

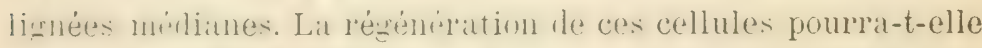

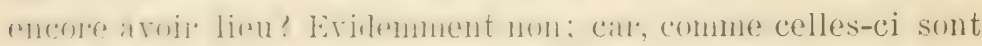

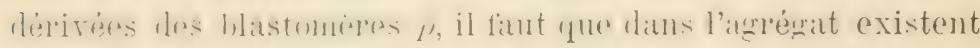

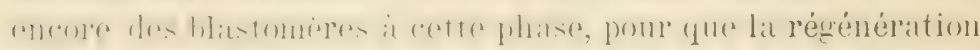

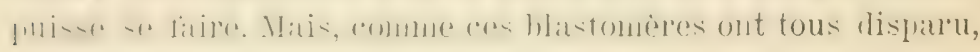
la régénération deriendra impossible. 
Ce que nous renons de supposer dans les premiers moments de l'ontogénèse pent arriver aussi dans los autres phases ultérieures; mais on connuremdral que cette imposibilité de régénération sera, dans lit plupart les cass, plus dillicilement réalisable.

Nous arons ru, en effet. qu'i mesure que le dreloppement progresse, les cellules qui sont le point de départ des nourelles lignées cellulaires deriennent plus nombreuses, de sorte que leur exportation complite de l'oriminisme est tris diflicile et, parfois, presque impossible.

Supposons, patr exemple, que les cellules tomant l'orane \%, dérivent d'une cellule f"., d"une lignée cellulaire précédente, et que celle-ci, à son tonr, lérive d'une série flus ou moins Inngue d'autres lignies cellulaire- Les cellules l' seront donc énmmément nombrures; et si tuntes ces cellule- ne donnaient origine qu'd̀ des lignées cellulaires $b_{0}, c_{0}, d_{0} \ldots p_{0}$ formant l'organe \%, on comprenlrait que, bien que l'on exportit cet organe, il y aurait toujours dans l'organisme quelques cellules à la phase $\mu^{\prime \prime}$ ou à d'autres phases précélentes pourant en produire la régénération.

Matis si l'on suppose yne les cellules l' ne soient pas toutes: le point le lépart de la seule lignée ho, co ... plo le l'organe o, et qu'au contraire elles puissent donner lieu, a cause du milien interne, a la formation d'autres lignees formant d'autres organes, on comprend facilement que le nombre des cellules $\mu_{2}$ pourant servil a la formation de l'organe () sera plus petit: car une partie de ces cellules servira it la froduction d'autres or fanes. Inans ce cas, la récénération de l'orrane osera moins probable, ou bien fourrat se faire un nombre de tois moindre, ou bien encore, l'organe r), qunique rigénéré, n’arrivera qu'i des dimensions plus petites que les normales.

('es ré-ultats nous démontrent, en conclusion, que le funvoir de régénération ext, at parite d'autres conditions, d'autant mointre que la ditremenciation de l'opranisme est plus gramle: car une plus grante diffërenciation ne peut se faire yu'aux 
lépens les cellules issues de la segmentation de l'oeuf, ce qui diminue nécessitirement le nombre de celles-ci pourant servir à la régénération.

Consirlérons maintenant l'importance du milieu interne.

Cous arons ru que l’érolution caractéristique de l'oeuf, c'esta-rlire la constitution bioplasmatique des cellules dérirant de sia sermentation, défrend non seulement de la constitution du bioplatma de loneuf, mais ausid de celle de son deutoplasma, écst-it-dire des som milien interne. I'où l'on comprend que le changrement de celui-ci doit entrin̂ner un changement dans son évolution.

Ce qu'on dit pour l'oeuf, on peut le répéter pour l'évolution de toutes les cellules.

si done, pour nous en tenir toujours à l'exemple cité de l'orgrane (), les cellules qui le forment ont une constitution bioplasmatique et, par suite, une différenciation histologiune léterminee, c'est que non seulement elles dérivent originairement l'une cellule d'une constitution bioplasmatique, elle aussi, déterminée, mais que le milieu interne, servant à l'assimilation de ces cellules, est, lui aussi, d'une composition chimique fixée.

Or, tant que ce milieu interne n'a pas changée, la régénération de l'organe pourra se fatre, pourvu, bien entendu, qu'il rxiste des cellules capables de cette rénénération, d'apris ce fue nous venons de démontrer. Mais si, lor's de l'exportation de l'organe, le milieu interne n'est plus parfaitement igal it celui dans lequel la formation de cet organe avait eu lieu, rien de plus naturel que la régénération ou bien ne puisse flus se faire, ou bien fu'elle aboutisie à la production d'un organe qui ne soït pas parfaitement identique à l'autre.

Dans toutes ces considérations, nous n'arons jamais tenu compte de la forme de loryane. Il nous reste done à examiner la régénération au point de vue morphologique.

Quelle est la cause de la forme déterminée d'un organe? Evidrament. la lorme, la strueture, et la disposition des élé- 
ments histologiques qui le composent. Or, la forme et la structure sont des caractères étroitement dépendants de la dillérenciation histologique des eléments. Mais la disyosition est en relation étroite arec les conditions mécaniyues dans lesquelles se troure l'organe lors de sa firmation. C'est un phénomene que l'on peut constater trè souvent dans l'éturle de lontogéndes des animaux, que les lois qui règlent la formation d'un organe au point de rue morphologique, peurent se ramener it des lois mécaniques, dépendant de la pression et de laadhésion des éléments de ces orranes ou des antres organes enriromnants. On comprend donc que, si ces conditions mécaniques spéciales n'existaient par, l'oratane en question ne prendrait pas sa forme caractéristique, bien que les éléments qui les constituent eussent acuuis, par leur érolution, la forme et la structure roulues.

Or, si la régéneration d'un organe quelconque a lieu dans des conditions mécaniques égales à celles qui existaient lors le sa formation, rien de plus naturel que l'organe régénéré snit égal, au point de rue morpholorgine ausisi, à l'organe exporté. Mais si les conditions mécaniques ne sont pas parfaitement les mènes. on concoit facilenent que la régénération ne puisse aboutir a la formation d'un organe morpholosiquement identique.

C"est, je croic, dans l'absence de ces conditions, qu'on duit chercher l'impossibilité de la rexénération morphologique de certaines parties lans quelques animaux. Dans ces cas, nous pourrons en effet constater que ce n'e-t pas la régénération histologique qui manque : car les tissus restants ont le pouroir de donner origrine it d'autres cellules d"une diflérenciation histolngique égale à celle des éléments exportés. Mais ces téments ne se disposent plus de la méme manière qu'anparavant, alor's qu'arait eu lieu la formation primitive de l'organe que nous considérons.

C'ertes, la connaisance exacte de ces comditions mécaniques est très diflicile, et parfois nume inposible. L'interprétation 
he la régénération derient donc, dans ces cas, un problème tres complexe, et d'une très grande difliculté; mais cela n'exclut pas que le principe et la cause du phénomène ne soient les mèmes yue daus los autres cas, tels que je riens de l'exposer.

le mème que les autres phénomènes de l'ontogénèse, ceux de la régénération constituent autant de problèmes complexes yu'il faut résoudre tour à tour, à mesure qu'ils se présentent, suirant les organismes que l'on consilere et après la connaissance exacte, autant que possible, des conditions dans lesquelles ils s'accomplissent.

D'ailleurs, ce n'est pas la solution de chacun de ces problemes que nous devons rechercher daus ce travail, mais la cause et le principe fondamental qui régissent et provoquent les phénomènes de régénération et nous permettent d'en donner une explication: ce que j'espère aroir démontré dans ce chapitre, en partant du déreloppement monodique, base essentielle du développement ontogénétique.

En resumé, nous pouvons conclure:

$1^{\circ}$ La régénération est un phénomène possible dans lous les organismes.

$2^{\circ}$ Elle n'est pas une faculté plus ou moins finaliste ou jur irlentielle, possédée par les êtres en lirérision des exigences de len, existence. Elle n'est qu'une 'onséquence directe, na-

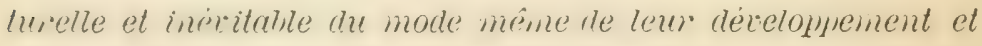
de leur constilution.

3० La régènérution est une conséquence du dérelonpenzent mannmlipue, que nous arons pose romme base rles thènomènes

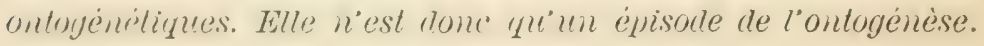




\section{Chapitre XVI.}

\section{L'ontogénèse des végétaux.}

SOMMAIRE: Analogio de développemont ontro los animaux ot les végétaux Parallèle entre l'oeuf des animaux et l'oenf' des plantes - Importance do la membrane dans la segmentation de l'oeuf et des cellules végétalos - Possi. bilité $d^{\prime}$ une explication do l'ontogénése des plantes par lo développement monodique - Le divelopmenent velique dans le developpement ultirieur des plantes.

L'interprétation que je riens de donner de l'ontogénèse des animaux peut aussi ètre aprlipuée a l'ontogénèse les régétaux. Cela d'allleurs ce concoit facilement, ru que les phénomenes fondamentaux sont a leu près les mèmes dians ces deux règnes de la Biologie.

L'onsphère fécondée, liveuf des plintes correspond sulstantiellement ì l'veuf' des animax, sauf, bien entendu, le dentoplasma uqu, comme nom: le sarons, n'existe pas dans les régétaux, ou cepenlant il est représente par des matériaux nutritifs du sac embryonnate, ou bien par des substances spéciales: albuninoides, amilon, sucre etc., dont se remplissent les cellules du suspensenr, ou lien encore par d'autres substances inconnues que les raneatux filitimes lu suspenseur puisent parfois directement dans le placenta.

Quoi qu'il en soit, toujours est-il que l'oeuf accomplit sa sormentation four des crombirises successires et, frar suite, pall des assinilations grialables dont les materiaux nicessaires sont fournis par l'individu producteur de l'vent, tout comme dans la segmentation de l'oeuf des animaux.

Lat formation du suspenseur ur complique nullonent le phé-

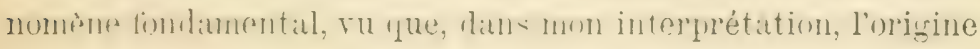


des diverses parties tient exclusivement it une senle cellule. Ia prolifération de l'oeuf froduit la première lignée cellulaire, formant ce qu'on appelle l'embryon, avec ou sans suspenseur. De toutes ces cellules, une seule se trouve, comme nous l'arons dómontré, à la phaso limite de lérolution de l'oenf; les autres se trouvent encore dans les phisses antérieures.

Ce yui constitue surtout une différence remarquahle entre cet agrégat do segmentation de l'oeuf des régétaux et l'agrégat de segmentation correspondant de l'oeuf des animaux, c'est la forme et l'absence d'une carité de segmentation.

Or, cette différence est une conséquence du mode de segrmentation, c'est-i-dire, des directions des plans de dirision des cellules.

Il fitut surtout remarquer, dans l'étude de la cýtodiérèse des végétaux, que les cellules le ces organismes possedlent presque toutes une membrane brute, grénéralement cellulosique, qui ne peut évidemment prendre part it la division cellulaire. Or, cette membrane se forme pendant la périorle assimilatrice de la cellule et, ce qui est de la plus haute importance, donne il lia cellule, pendant sa formation, une forme bien déterminée, dépondant ou bien de la nature même de la cellule, ou bien de celle-ci et des causes mécaniques externes pourant agir sur elle, ou bien encore de causes spéciales intimes physico-chimiques que nous ne pourons connaitre exactenent. (vuoi qu'il en soit, la formation de cette nembrane et la forme conséquento spéciale de la cellule ont une action, sans aucun doute, capitale dans la direction des plans de dirision, ainsi que je l'ai fait ressortir dans la l" partie de cet ourrage (S IV, p. 212).

Je ne puis ériłemment marriter ici ì considérer tous les cas frossibles relatifs a ces diverses directions par rapport ì lat forne de la cellule. Je suis forcé de renroyer le lecteur i l'exanen des prohlènes andytirues de la cytoliérèse. Mais je suis convalincu, ot jespire que les résultats des observations confirmeront mes convictions, due, dans chacun de ces cas 


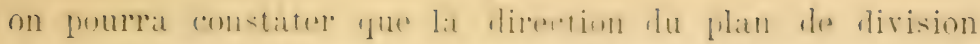
n'est qu'me conséquence lese conditions méciniques de lat cellule, et quelle peut ire tumjours exactenent déterminée, si lon connat exactement ansi lat valeur de ces conditions.

l)'ailleur's, nous l'arons vu, lat forme de l'agregat de segmentittion n'a qu'une importance tout-ì-fait secondaire dans le développenent ontogénétique, daprès notre interprétation.

Quant at laabsence de lat carité le segmentation, elle n'exclut fals, ain-i qu'il paraitrait le prime abord, la présence d'un liquide te sermentation, c’est-it-dire he substances spéciales Iroduites par la sécrétion des cellules de la première lig̣née. Ces substances peurent hien aroir été prorluites par ces cellules et itre contenues dans lo sac embryonnaire dans lequel est plongé l'embryon même.

s'il en est ainsi, et c'est très probable, nous arons, dans l'arrégat de sermentation des rérétaux, une cellule à la phase linite de l'érolution de l'venf', et drs substances fourant selrir' il la mutrition de cette cellule et froroquer la formation d'une deuxième lignée cellulaire. Iès lors, nous y arons des conditions parfaitement analugues a celles consilérées dans l'ontogénèce les aninaux, et les phénmènes fourront, par suite, se passer comme chez ces derniers.

D'ailleurs, d'autres recherches minutieuses et rignureuses, et surtout des recherches l'embryolugie experinentale (si toutefois celles-ci sont possible: dins les regrtaux) sont encore nécessaires four arriver it dos conclusions rraiment scientiflupes sur le dévelupenent de ces organismes. Ici encore, comme dans les animanx, je crois que, hien que le principe fondamental de l'ontugréness wit le mène, le dévelorpement des diverses espoces ennstifue autant de frollemes qui doivent itre résulus séparénent, lapprès les donnes des conditions spéciales argissant sur le déreaplyenent et fouvant varier dans chaque espèce.

La formation de la radicule. les cotylédons et du crine rérétatif ne présente, l'autre part. aucune lifficulté à une 
explication, d’apres mon interpreitation. Fous y tromvons des phénomènes analogues an díreloppenent des animaux, où l'on Foit se produire les organes caractérisant les parties antérieure, postérieure et latérales.

Le léveloppenent ultérieur le l'embryon peut, au contraire, donner lieu à quelque riscussion sur son interpretation.

Jous sarons que la cellule ou les cellules apicales produisent, par des segmentations répétées, plusieurs autres cellules constituant les tissus de la tige, et que certaines de ces collules, par d'autres segmentations, produisent les feuilles.

Je ferai arant tout remarquer que le type de division des cellules apicales peut se ramener, ainsi que je pourrais le démontrer, aux lois que j’ai énoncées daus la prenière pantie de cet ourrage, arec les solutions des problemes analytipues le la cytodiérèse, pourru que l'on tienne toujours compte de la forme de ces cellules. déterminée par la membrane cellulosique qu'elles présentent.

Mais ce qu'il importe surtout d'expliquer dans ce cas, c'est li lisposition spéciale que les feuilles présentent sur li tiğe et la répétition de leur proluction le long de la tigre même.

Nous pouvons recourir, dans ce cas, si nous le voulons, au héreloppenent cyclique, tel que je l'ai exposé au chapitre XIV de cette partie. Tous pourons supposer, par exemple, que la cellule apicale du cone régétatif emhryonnaire, après un certain nombre de divisions suivant le développement monodique, peut Ionner lieu it deux cellules, dont une soit égale, par sat constitution bioplasmatique, à la cellule apicale même qui ì été le point de départ de la prolifération. En indiquant par $a^{\prime}$ cette cellule apicale, et par $b^{\prime}, c^{\prime}, d^{\prime}, e^{\prime} \ldots p^{\prime}$ les autres cellules, on arriverait, dans cotte supposition, a obtenir, comne proluits de la division de la cellule r', deux cellules, dont une i' et l'autre $a^{\prime}$. De cette maniere, celle-ci, se trourant dans des combitions igales a celles dans lesquelles se trourat la jermide cellule apicale r', sorait naturellement capable de surre la mrime irolution, en domnant lieu il d'autres cellules et 


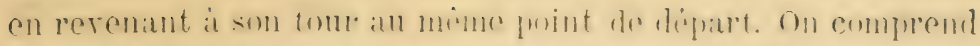

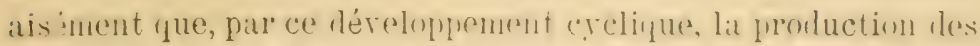
fonilles pourrait se filire indéfiniment.

bien que, par ce mole de léveloppenunt, on arrive à domer une explication apparemnent satisfaisante du phémomène en question, je crois néanmoins que les plénomènes ne se passent pas réellement le cette maniele.

En ellet, si le lérulupument stait rrament cyclique, le nombre des temilles derrait are indéfini ot les feuilles produites par la tige, dans les diverses périoles re sa vie, dovraient ètre tuntes identiques entre elles, ce que je ne crois pas qu'on puisse aflirmer.

Cette irlentiti parfiate les teuilles est loin l'itre démontrée. Jo crois, atl contritre, qu'un exanem, mème superficicl, l'une plante quelemune est sullisunt pour mons prourer que cette irlentite n'existe pas. (guoique apparemuent semblables datns certitins cas, les feuilles des plantes ne sont pas toutes identiques, mais liflerent au contraire entre clles, soit par leur forme, soit par leur constitution interne ou externe.

l'alutre part, le nomhre des feuilles, bien que très mand, et parfois extraordinaire, n'est pas indéfini.

si les limites de la promuction des feuilles ne neurent fas itre connues dans les plantes qui ont une duree de vie tres: longue, il n'est pats riflucile de la constater dans colles oil lat durée de la vie est courte. Mais personne ne pourra affirmer que la durée de la rie de certain's plantes, quoique trés longrue, soit inbéfinir. Il est, all contraire, tres [robable et, je dirai mome tres sim, que cette durée est limitee, bien que ces limites soient tellement éloginées quelles échappent at motre observation directe. Or, s'il en ext ainsi, le nombre des - feuilles, doit, Iui aussi, être défini.

Cer consiblérations ne me pernettent pas de croire que de

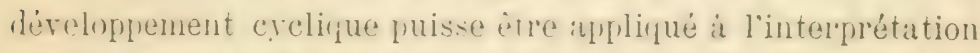
du déreloppement des régétaux. Je ne crois gas que les cellules apicales de la tige reriennent janais a leur rrai point 
de lepart: matis je pense, alu contribe, que les cellules dérivant de lat sermentation des cellules apricales constituent par leur ensemble une série continue, sans revenir jamais at une constitution bioplasmatique parfaitement identique a la cellule initiale.

On expliquerait par lit pouryuoi le nombre des fouilles ne peut ètre indéfini, et l'on comprendrait encore que, la série des rellules derant aroir des limites, la durée de la rie des végrétaux doit ètre nécessairement limitée, tout comme celle des animaux.

Je n'insiste pasi davantage sur cette question, d'autant plus que j'ai l'intention d'y revenir plus tard dans une partie le cet ourrage. Mais j'espère que mon interprétation de l'ontogénèse des animaux pourra engager des savants botanistes a l'appliquer aux régétaux, et que cette application, faite par des biologistes possédant des connaissances profondes qui, malheureusement, me manquent dans ce règne de la Biologie, ahoutira à des résultats satisfaisants.

Il s'agit maintenant le roir comment se produit l'oeuf, en quoi consiste la fécomlation, et pourquoi les individus issus de l'veuf présentent les caracteres de leurs parents. C'est ce que nous verrons daus la III" Partie de cet ourrage: LA FÉCONDATION ET L'HÉRÉDITÉ.

Fin de ia DEUXiène PARTIE. 




Giglio-Tos, E.

Les Problemes de la vie.
QH

331

. 455 


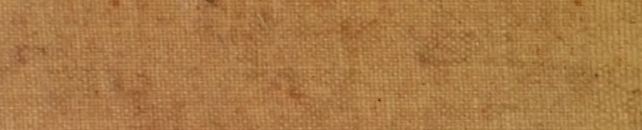

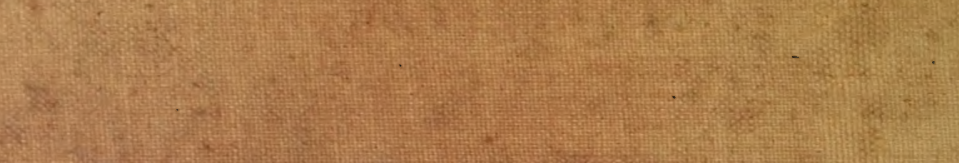

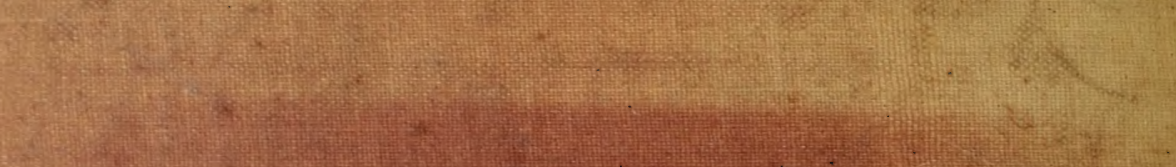

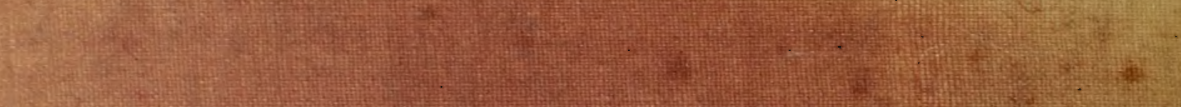

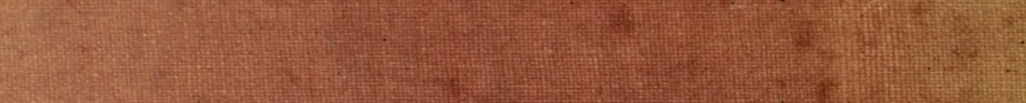

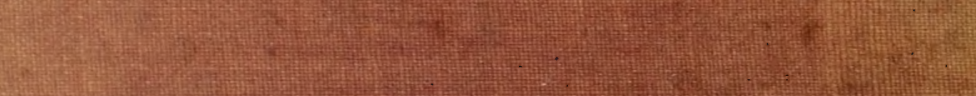

4.2.

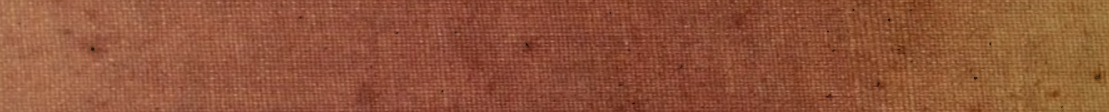

(1.

(2)

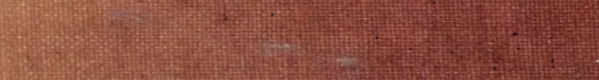

20.0.

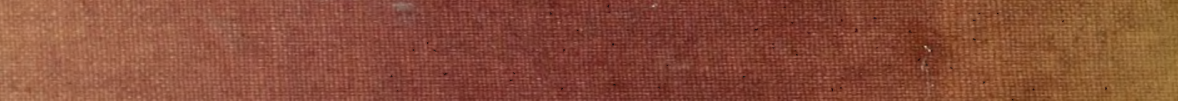

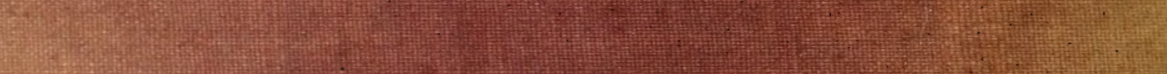

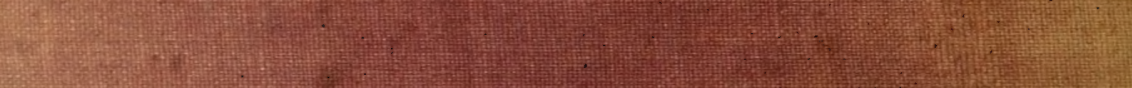

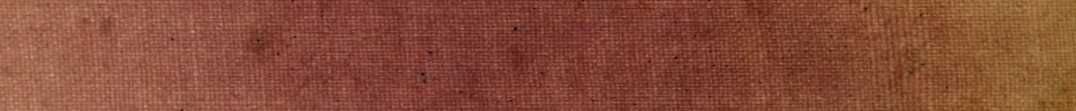

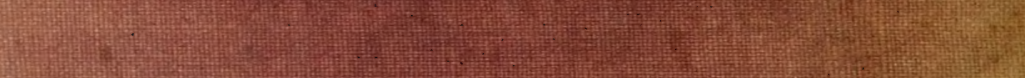
(2)

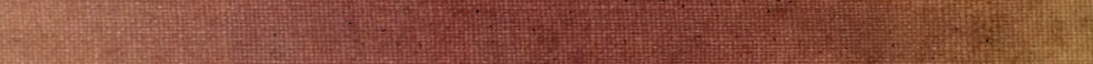

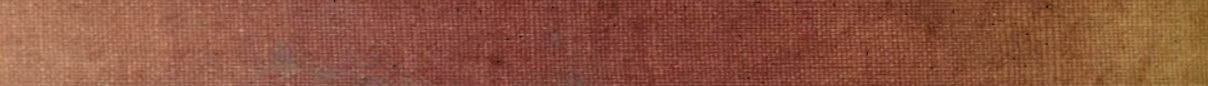

(2)

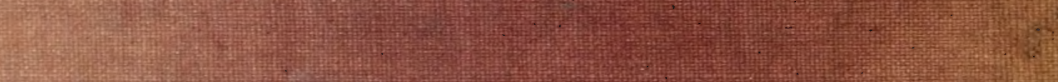

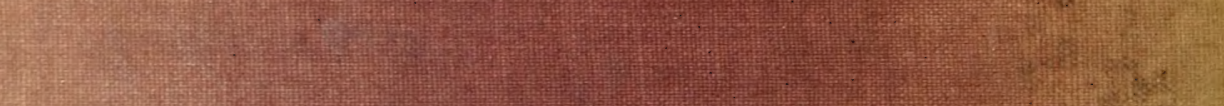

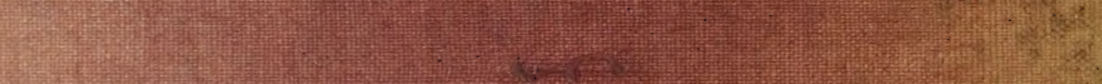

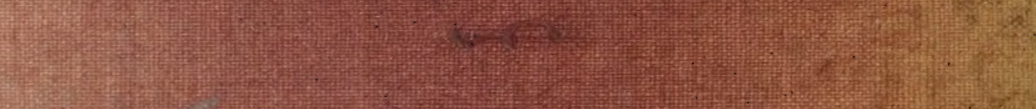

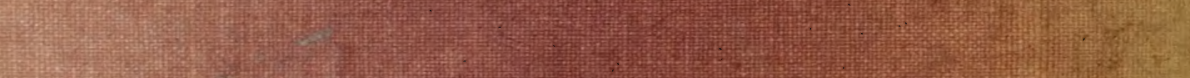

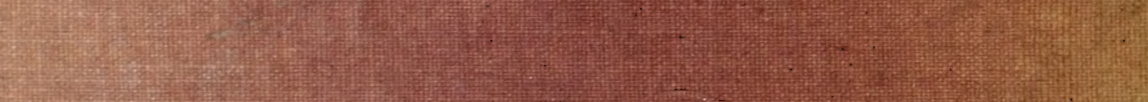

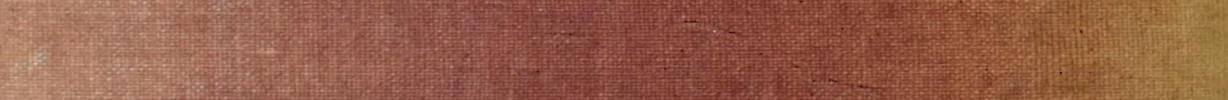

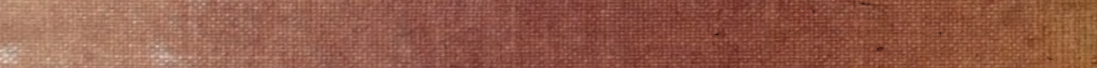
2. $\sin 20=0$ 